CHECIT PL 68-1

CALIFORNIA INSTITUTE OF TECHNOLOGY

CHEMICAL ENGINEERING - POLYMER LABORATORY

\title{
A RESEARCH PROGRAM ON
}

SOLID PROPELLANT PHYSICAL BEHAVIOR

N. W. Tschoeg1

Project Director

R. F. Landel

Senior Investigator

H. Kausch, C. K. Lim, R. G. Mancke

D. G. Fesko, K. Yagii

Investigators

AFRPL-TR-68-106

JUNE 1968

First Annual Report

1 February 1967 - 30 April 1968

Contract Number F04611-67-C-0057

Air Force Rocket Propulsion Laboratory

Research and Technology Division

Edwards Air Force Base, California 93523

Air Force Systems Command

United States Air Force

This document is subject to special export controls and each transmittal to foreign governments or foreign nationals may be made only with prior approval of

AFRPL(RPPR-STINFO), Edwards, California 93523

EUDORA HULL SPALDING LABORATORY OF ENGINEERING

Pasadena, California 91109 


\begin{abstract}
Release of this document by the Defense Documentation Center to the Clearing House for Federal Scientific and Technical Information is not authorized.
\end{abstract}

When U. S. Government drawings, specifications, or other data are used for any purpose other than a definitely related Government procurement operation, the Government thereby incurs no responsibility nor any obligation whatsoever, and the fact that the Government may have formulated, furnished, or in any way supplied the said drawings, specifications, or other data, is not to be regarded by implication or otherwise, or in any manner licensing the holder or any other person or corporation, or conveying any rights or permission to manufacture, use, or sell any patented invention that may in any way be related thereto. 


\section{PREFACE}

This report presents the results obtained during the past year in a research program on Solid Propellant Physical Behavior carried out at the California Institute of Technology with the support of the U.S. Air Force Rocket Propulsion Laboratory, Lt. Scott Beckwith, Project Engineer, under Contract No. F-4611-67-C-0057. The object of the research program is to contribute to our understanding of the physical behavior of solid propellants through a comprehensive study of filled elastomeric systems.

The report is presented in eight independent parts which may be grouped conveniently as follows:

Parts I - III deal with theoretical and experimental investigations of the physical properties of filled systems and binders:

I Failure Criteria for Filled and Unfilled Elastomers C. K. Lim and N. W. Tschoegl

II On the Packing of Spheres of Different Diameters in Space H. Kausch and N. W. Tschoegl

III Mechanical Properties of Polymer Blends D. G. Fesko and N. W. Tschoegl

Parts IV - V report on further progress on the Standardized Rubber Program:

IV Further Characterization of GALCIT I R. F. Landel

V Finite Deformation Studies

R. F. Landel and R. G. Mancke

Parts VI - VIII describe studies relating to the mathematical theory of linear viscoelasticity.

VI Approximation Methods for the Determination of the Spectral Distribution Functions from Experimental Response Functions

N. W. Tschoegl 
VII The Application of the z-Form Numerical Method of Inverse Laplace Transformation to Problems in

Linear Viscoelasticity

D. G. Fesko

VIII Response of Viscoelastic Materials to Piecewise Continuous Excitations

K. Yagii and N. W. Tschoeg1

Each part has its own table of contents, list of illustrations and tables, references, and figures and tables.

This report is issued as CHECIT PL 68-1, AFRPL-TR-68-106.

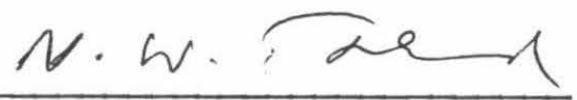

N. W. Tschoegl

Professor, Chemical Engineering, Project Director

This technical report has been reviewed and is approved.

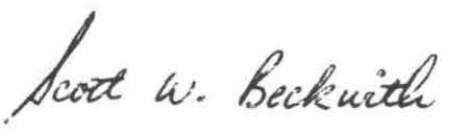

Scott W. Beckwith

1st LT, USAF

Project Engineer 


\section{A BSTRACT}

Part I reports on further progress in a program to generate failure data on filled and unfilled elastomers in the compressive octants of principal stress space. Rubber rings swollen in hydrocarbon solvents were stretched in uniaxial tension at a crosshead speed of 1.45 inches per minute under superposed hydrostatic pressures up to $2000 \mathrm{psig}$. Two elastomers were examined: a moderately cross-linked compression molded styrenebutadiene rubber, and the interim standardized rubber, GALCIT I, a more tightly cross-linked cast polyurethane rubber. Both materials obeyed the stress-strain relation predicted by the kinetic theory of rubber elasticity under all pressures. The tests were carried out at room temperature and were corrected to $25^{\circ} \mathrm{C}$. The superposed hydrostatic pressure had no effect on the tensile strength of the two rubbers. Within the experimental scatter the stress-at-break appeared to depend on the octahedral shear stress alone and thus followed the Huber-Hencky-von Mises failure criterion. The failure surface of the swollen rubbers in principal stress space could therefore be described by a cylinder coaxial with the hydrostatic axis.

Part II reports on an attempt to derive the optimal distribution of sizes of randomly aggregated spheres as a first step in the development of a theory of optimum packing. It is shown that an investigation of the interstices in a lattice of regularly packed spheres does not yield an unambiguous distribution over a wide range of sphere sizes. The corresponding problem in the plane, however, could be studied unambiguously. The results obtained in such a study are useful because of the equivalence of volume, area, and line fractions of random assemblies. An optimal distribution of circle sizes, $n(r)$, for the regular packing of circles in a plane was derived. The value of $\mathrm{n}(\mathrm{r})$ in random packing of circles is discussed and a method is 
developed to transform $\mathrm{n}(\mathrm{r})$ into the corresponding distribution $\mathrm{N}(\mathrm{R})$ of sphere sizes. $N(R)$ is obtained in form of a histogram. It still remains to be shown experimentally whether sphere distributions of the calculated type do indeed result in maximum packing fractions and optimize mechanical properties of filled elastomers.

A research program to investigate the blending of two homopolymers with the aid of diblock copolymers is outlined in Part III. The thermodynamics of the separation of polymers in blends, and the synthesis of block copolymers by means of anionic polymerization are discussed. The program is to include microscopic examination of a sequence of ternary mixtures of two homopolymers with their diblock copolymer to determine the variation in domain size of the separated components. The blends will then be characterized mechanically by measuring the dynamic shear modulus. These studies are expected to contribute to our knowledge of binderfiller interaction, and may lead to novel ways of binder formulation.

Part IV deals with characterization studies in the area of the standardized rubber program. The measurements of the physical properties of the Solithane 113 elastomers, including the interim standard rubber, GA LCIT I, were continued. Sol-gel and compressive stress-strain measurements in the swollen state showed that there was little sol at any composition from 50 to 80 volume percent of prepolymer and that the effective chain concentration was a constant over this composition range. A further analysis of the ultimate properties measured in uniaxial extension, in conjunction with these results, showed that the increase in prepolymer content lowers the number of equivalent random links per chain from 12 to 4.2. The polymer-solvent interaction parameter $\chi$, was evaluated as a function of the degree of swelling in five solvents. The solubility parameter 
was found to be $11.6\left(\mathrm{cal} / \mathrm{cm}^{3}\right)^{\frac{1}{2}}$. The Mooney-Rivlin $C_{z}$ parameter is essentially zero at room temperature. An independent measurement of Poisson's ratio as determined in the poker chip test at $5 \%$ compressive strain gave a value of 0.4996 , which compares reasonably well with a previous value of 0.4998 .

Part V reports on finite deformation studies. Large-strain properties of natural rubber cross-linked with $1 \%$ sulfur were studied in an attempt to further our understanding of the strain-energy function of that material. The Becker-Landel torsion-tension tester was used to measure the torsional and axial forces required to support combined twist and extension, or compression, of more than 50 solid cylindrical rubber specimens of three different radii. In the cases of combined extension and twist the forces at five minutes after application of the deformation were found to follow equations derived from the Signorini form of the strain-energy function, with appropriate values of the three material constants. Further work showed, however, that the values of the twist variable were in error to some extent, and further discussion will be deferred until correction of these errors.

Part VI describes a continuation of earlier investigations of approximations to the spectral distribution functions of linear viscoelastic theory. It is shown that if the order of the highest derivative is $k$, then there exist $k$ approximations from the step and the storage functions, and $k+1$ approximations from the loss functions. Thus a number of new, hitherto unknown, approximations are found, which include approximations of even order from the storage functions, and approximations of odd order from the loss functions. Of particular practical interest are the two approximations of first order from the loss functions. The inter-relation between the $k$ 
approximations of a given order, and the choice of the most suitable approximation is discussed. The relation of the more general method to the transform inversion method is examined, and it is shown that certain approximations in the current literature are in error because of lack of normalization of the associated intensity function, and, where applicable, because of failure to take into account that the time scale of the spectrum must be shifted by the appropriate amount if the maximum of the intensity function does not lie at the origin. Finally, the derivation of approximations from creep recovery, stress relaxation after cessation of steady flow, and deformation at constant rates of strain is discussed.

In Part VII the $\mathrm{z}$-transform method for the numerical inversion of the Laplace transform is examined for possible application to viscoelastic problems. The method is found to be unsuited for viscoelastic functions which are given as logarithmic functions of time or frequency, because the logarithmic spacing of the variables leads to unstable solutions.

Part VIII predicts a general method for expressing the response of viscoelastic materials to the piecewise continuous excitation functions to which they are subjected in cumulative damage testing, as functions of the standard response functions such as relaxation modulus and creep compliance, or, in case of periodic excitations, the dynamic moduli and compliances. It is expected that departures from the predicted behavior can be ascribed to cumulative damage. This could lead to the development of a non-destructive fatigue tests. 
CONTENTS

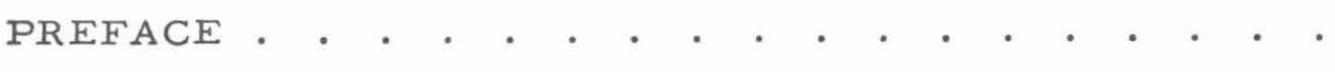

A BSTRACT.

PART I FAILURE CRITERIA FOR FILLED AND

UNFILLED ELASTOMERS . . . . • . . . . . I-1

PART II ON THE PACKING OF SPHERES OF

DIFFERENT DIAMETERS IN SPACE . . . . . II-1

PART II MECHANICAL PROPERTIES OF

POLYMER BLENDS . . . . . . . . . . . . III-1

PART IV FURTHER CHARACTERIZATION OF

GALCIT I . . . . . . . . . . . . . . $\quad$. IV -1

PART V FINITE DEFORMATION STUDIES . . . . . . $\quad$ V-1

PART VI APPROXIMATION METHODS FOR THE DETERMINATION OF THE SPECTRAL DISTRIBUTION FUNCTIONS FROM

EXPERIMENTAL RESPONSE FUNCTIONS . . . VI-1

PART VII THE APPLICATION OF THE Z-FORM

NUMERICAL METHOD OF INVERSE

LAPLACE TRANSFORMATION TO

PROBLEMS IN LINEAR VISCOELASTICITY . . . VII-1

PART VIII RESPONSE OF VISCOELASTIC MATERLALS

TO PIECEWISE CONTINUOUS EXCITATIONS . . VIII- 1 

PART I

FAILURE CRITERIA FOR FILLED AND UNFILLED ELASTOMERS

C. K. Lim and

N. W. Tschoegl 
PART I

FAILURE CRITERIA FOR FILLED AND

UNFILLED ELASTOMERS

\section{CONTENTS}

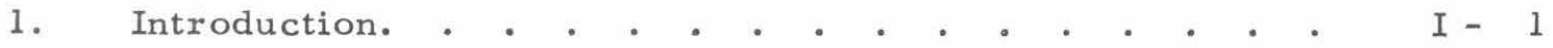

2. Failure Surfaces in Principal Stress Space for

Polymeric Materials . . . . . . . . . . . . I - 3

2.1 Plastics . • • . . . . . . . . . . . I 1

2. 2 Unfilled Elastomers . • . . . . . . . . . I - 10

2.3 Foams . . . . . . . . . . . . . . . I I 14

2. 4 Filled Elastomers and Propellants . . . . . . I - 15

2.5 Conclusions. • . . . . . . . . . . . . . I - 19

3. Tensile Strength and Stress-Strain Behavior of Swollen

Elastomers under Superposed Hydrostatic Pressure . . I I 19

3.1 Description of Apparatus . . . . . . . . . I 21

3. 2 Calibration and Experimental Procedure . . . . I - 25

3. 3 Uniaxial Extension Tests on Rubbers under Superposed Hydrostatic Pressure . . . . . . . . . I - 28

3. 4 Effect of Hydrostatic Pressure on Modulus . . . I - 29

3. 5 Effect of Hydrostatic Pressure on the Ultimate
Properties. . . . . . . . . . . I - 32

3. 6 Failure Surfaces for the Swollen Elastomers. . . I - 37

References . • • . . . . . . . . . . . . . I 43 


\section{ILLUSTRATIONS}

Fig. 1. Comparison of Biaxial Failure Criteria (Marin $\left.^{(4)}\right)$.

Fig. 2. Failure Surfaces in Principal Stress Space

(Tschoeg $\left.{ }^{(1)}\right)$. a) Cylinder, b) Cone, c) Paraboloid . I - 7

Fig. 3. Intersection of Failure Surfaces with $\sigma_{1}=\sigma_{2}$ Plane (Tschoegl $\left.{ }^{(1)}\right)$. a) Cylinder, b) Cone, c) Paraboloid . I - 7

Fig. 4. Fracture Stress for Poly (methylmethacrylate)

(Thorkildsen and Olszewski $\left.{ }^{(6)}\right)$. . . . . . I - 8

Fig. 5. Biaxial Fracture of Nylon $\left(\mathrm{Ely}^{(7)}\right)$. . . . . . . $\quad$ I - 9

Fig. 6. Biaxial Fracture of Acrylic $\left(E l y{ }^{(8)}\right)$. . . . . . I 10

Fig. 7. Biaxial Failure of SBR (Smith and Rinde ${ }^{(10)}$ ) . . . I I 11

Fig. 8. Biaxial Failure of Paracril (Lim $\left.{ }^{(1)}\right)$. . . . . . I 12

Fig. 9. Biaxial Failure of Solithane $\left(\operatorname{Lim}{ }^{(11)}\right)$. . . . . I 13

Fig. 10. Failure Surface of Polyurethane Rubber in First

Octant of Stress Space $\left(\mathrm{Ko}^{(12 \mathrm{~b})}\right)$. . . . . . . I - I4

Fig. 11. Failure Surface of Polyurethane Foam Rubber in

First Octant of Stress Space (Blatz ${ }^{(14)}$ ) . . . . . I I 15

Fig. 12. Biaxial Failure Behavior of Filled Hycar

(Sharma and $\operatorname{Lim}^{(15)}$ ). . . . . . . . . . I I 16

Fig. 13. Failure Surface of a Hydrocarbon Propellant in First

Octant of Stress Space (Jones and Kruse ${ }^{(16)}$ ) . . . I - 17

Fig. 14. Total Uniaxial Stress at Break of a Compressible (A) and an Incompressible (B) Hydrocarbon Propellant under Superposed Hydrostatic Pressure (Jones and Kruse ${ }^{(16)}$ ) . . . . . . . . . . . I I - 18

Fig. 15. Schematic of the Pressurized Tensile Tester . . . I - 20

Fig. 16. Piping Schematic for the Pressurized Tensile 
Fig. 17. Electric Schematic for the Pressurized Tensile

Tester. . . . . . . . . . . . . . . I I - 24

Fig. 18. Calibration of Crosshead Displacement Monitor . . I - 25

Fig. 19. Experimental Arrangement for Calibration of the

Load Cell . . . . . . . . . . . . . . I 26

Fig. 20. Calibration of Load Cell Output. . . . . . . . I I 26

Fig. 21. Effect of Hydrostatic Pressure on the Modulus of

Swollen SBR. . . . . . . . . . . . . . I - 30

Fig. 22. Effect of Hydrostatic Pressure on the Modulus of

Swollen GALCIT I . . . . . . . . . . . . I I 31

Fig. 23. Effect of Hydrostatic Pressure on the (Neohookean)

Tensile Strain at Break for Swollen SBR and GALCIT I I - 35

Fig. 24. Effect of Hydrostatic Pressure on the Tensile Stress at Break for Swollen SBR . . . . . . . . . I - 36

Fig. 25. Effect of Hydrostatic Pressure on the Tensile Stress at Break for Swollen GALCIT I. . . . . . . . I - 36

Fig. 26. Effect of Hydrostatic Pressure on Equilibrium

Stress-Strain Curve for Swollen SBR at $25^{\circ} \mathrm{C}$. . . I - 38

Fig. 27. Effect of Hydrostatic Pressure on Equilibrium

Stress-Strain Curve for Swollen GALCIT I at $25^{\circ} \mathrm{C}$. I - 38

Fig. 28. Failure Stress of Swollen SBR in $\sigma_{1}=\sigma_{2}$ Plane . . I - 39

Fig. 29. Geometric Representation of Isotropic and

Deviatoric Stress in Principal Stress Space . . . I - 41

Fig. 30. Plot of Deviatoric against Isotropic Failure Stress

for Swollen SBR and GALCIT I . . . . . . . . I I 41 
TABLES

Table I Statistics for Pressurized Tensile Tests for Swollen SBR Rings . . . . . . . . . . . . I - 33

Table II Statistics for Pressurized Tensile Tests for Swollen GALCIT I Rings . . . . . . . . . . . . . I - 34 


\section{Introduction}

The proper design of a solid propellant rocket motor requires knowledge of the ultimate properties of the propellant under multiaxial loadings. The multiaxial failure criterion may be expressed geometrically in the form of a failure surface in principal stress space. In an isotropic material this surface is symmetric with respect to the space diagonal, i. e. the axis along which the three principal stresses are equal. Any combination of stresses is safe if the corresponding point in principal stress space falls within the failure surface.

The material will fail if the point lies on or outside of the surface. In principle, the failure surface may be determined experimentally by carrying out a sufficient number of tests under appropriate multiaxial loading conditions. An analytic representation of the surface can then be found, and this may serve as the failure criterion.

During the previous contract period (cf. previous annual report ${ }^{(1)}$ ) a program was initiated for the experimental establishment and theoretical interpretation of failure surfaces in principal stress space for filled and unfilled elastomers. The theory of the simplest failure surfaces in principal stress space was discussed, and a program was developed to generate data in the compressive octants of stress space (in which at least one stress is negative) by carrying out tests in uniaxial extension and in pure shear under a superposed hydrostatic pressure. An apparatus was designed for this purpose and its construction was begun. For reasons explained more fully in the previous report ${ }^{(1)}$, experiments were to be made initially on swollen rubbers. 
During the first contract year information was obtained on the behavior of swollen styrene-butadiene rubber (SBR) in uniaxial extension and compression under atmospheric pressure. The stress-strain behavior of the swollen SBR could be described by the kinetic theory of rubber elasticity (neohookean theory) up to the point of break. Break data obtained in uniaxial extension on swollen rings at different strain rates and at different temperatures, when corrected by the temperature correction provided by the theory of rubber elasticity, scattered around a straight line representing the (neohookean) equilibrium stress-strain curve. Because of the time-independence of the swollen rubbers ${ }^{(1)}$, the curve could also be regarded as the Smith failure envelope for the swollen material. The modulus calculated from the slope of the line of best fit through the break data was in excellent agreement with the mean modulus obtained by averaging the slopes of the individual stress-strain curves.

Tests at room temperature on swollen SBR cylinders evinced the same behavior. The uniaxial compressive break stress was higher than the uniaxial tensile break stress. This indicated that hydrostatic pressure could be expected to have an effect on the failure behavior.

During the present report period the construction, assembly and installation of the apparatus were completed. The instrumentation was added, and the system was checked out and calibrated. Tests were made at room temperature on two swollen elastomers, a moderately crosslinked SBR, and the highly crosslinked interim standardized rubber, GA LCIT I, under pressures ranging from 0 to 2000 psig. Section 2 of this report briefly surveys what is known of the multiaxial failure behavior of polymeric materials. Section 3 deals with the experimental work. It describes the experimental method, and the results obtained in the tests. 


\section{Failure Surfaces in Principal Stress Space for Polymeric Materials}

Most of the presently available information on the failure behavior of polymeric materials is derived from the uniaxial extension test. Failure criteria for uniaxial failure (such as the well-known Griffith-Orowan-Irwin criterion) attempt to predict the uniaxial break stress from parameters such as Young's modulus, crack propagation energy, and crack length. Criteria for failure under multiaxial stress-strain loadings are expressed in a more indirect way in terms of the failure behavior under uniaxial loadings.

Such multiaxial failure criteria were historically developed to characterize the biaxial failure of metals, glasses, and ceramics. The corresponding theories are known as the maximum stress, maximum shear, maximum strain, maximum strain energy, and distortion energy theory. The associated biaxial failure criteria may be represented as failure areas in principal stress plane as shown in Fig. 1, taken from Marin (4), where use was made of the stress-strain relations of infinitesimal theory of elasticity. The failure theories are adequately described in a number of publications $(2,3,4,5)$. The simplest of the biaxial criteria represent polygonal failure surfaces. Since such behavior is physically unlikely, these theories must be regarded at best as approximations. Generalization to three dimensions is straight forward, and leads, for the simpler theories, to polygonal failure surfaces.

In the discussion of failure surfaces in principal stress space presented in the last report ${ }^{(1)}$ only those criteria were considered which contained the three principal stresses in a symmetric fashion. This makes the corresponding failure surfaces in stress space surfaces of revolution 


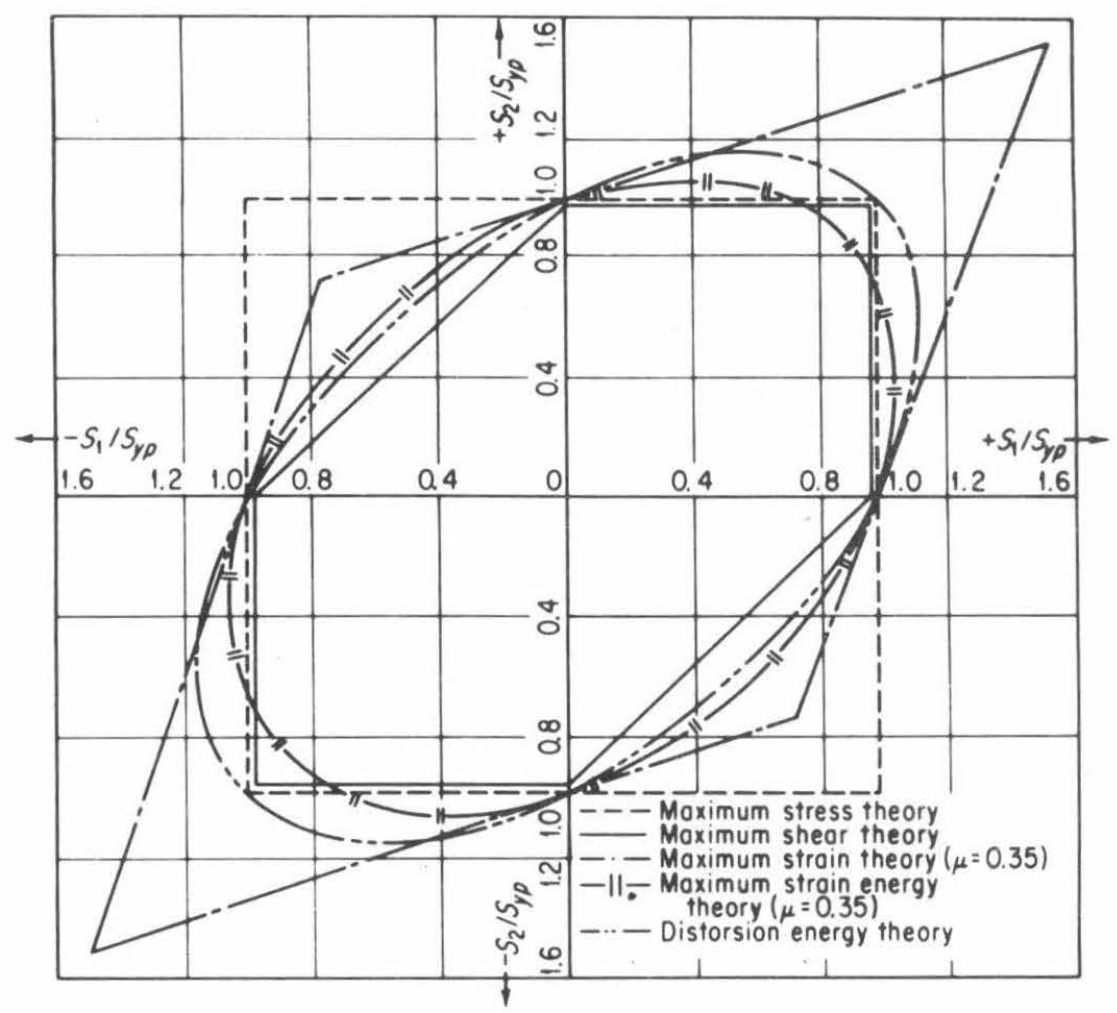

Fig. 1. Comparison of Biaxial Failure Criteria (Marin ${ }^{(4)}$ ).

around the space diagonal $\sigma_{1}=\sigma_{2}=\sigma_{3}$ as the symmetry axis. It was shown that the simplest such surfaces were the cylinder, the cone, and the elliptic paraboloid. For convenience the resulting criteria are summarized below:

\section{1) Cylinder}

$$
\sigma_{\mathrm{os}}=\frac{\sqrt{2}}{3} \sigma_{\mathrm{Tb}}
$$

2) Cone

$$
\frac{1}{\sqrt{2}}\left(\sigma_{\mathrm{Cb}}+\sigma_{\mathrm{Tb}}\right) \sigma_{\mathrm{os}}+\left(\sigma_{\mathrm{Cb}}-\sigma_{\mathrm{Tb}}\right) \sigma_{\mathrm{on}}=\frac{2}{3} \sigma_{\mathrm{Cb}} \sigma_{\mathrm{Tb}}
$$


3) Cone of Maximum Angle

$$
\frac{1}{\sqrt{2}} \sigma_{\text {os }}+\sigma_{\text {on }}=\frac{2}{3} \sigma_{\mathrm{Tb}}
$$

4) Paraboloid*

$$
\sigma_{\mathrm{OS}}{ }^{2}+\frac{2}{3}\left(\sigma_{\mathrm{Cb}}-\sigma_{\mathrm{Tb}}\right) \sigma_{\mathrm{On}}=\frac{2}{9} \sigma_{\mathrm{Cb}} \sigma_{\mathrm{Tb}}
$$

5) Plane

$$
\sigma_{\text {on }}=\frac{1}{3} \sigma_{\mathrm{Tb}}
$$

where

$$
\begin{aligned}
\sigma_{1}, \sigma_{2}, & \sigma_{3}=\text { principal stresses } \\
\sigma_{\mathrm{Tb}} & =\text { failure stress in uniaxial tension } \\
\sigma_{\mathrm{Cb}} & =\text { failure stress in uniaxial compression } \\
\sigma_{\mathrm{os}} & =\text { octahedral shear stress } \\
\sigma_{\text {on }} & =\text { octahedral or mean normal stress }
\end{aligned}
$$

The two octahedral stresses are given in terms of the principal stresses

by

$$
\sigma_{\text {os }}=\frac{1}{3}\left[\left(\sigma_{1}-\sigma_{2}\right)^{2}+\left(\sigma_{2}-\sigma_{3}\right)^{2}+\left(\sigma_{3}-\sigma_{1}\right)^{2}\right]^{\frac{1}{2}}
$$

and

$$
\sigma_{\text {on }}=\frac{1}{3}\left(\sigma_{1}+\sigma_{2}+\sigma_{3}\right)
$$

* The equations (29) and (30) of the previous report ${ }^{(1)}$ describing the ellipse generated by the intersection of this paraboloid with the $\sigma_{1}=0$ plane have sign errors. The first plus signs in the fourth terms of the equations should be minus. 
The cylindrical surface is the geometric representation of the distortional strain energy criterion, also known as the Huber-Hencky-von Mises criterion. Its association with the distortional strain energy is based on small strain theory. This is not valid for materials which undergo large deformations before break, and this theory will be referred to here as the octahedral shear stress theory, as proposed by Nadai (2). According to this criterion, only the octahedral shear stress determines failure, the mean normal stress is without effect.

The criterion represented by the cone is essentially the threedimensional form of the Coulomb-Duquet-Navier criterion (1), in which the maximum shear stress is replaced by the octahedral shear stress, and the coefficient of internal friction is expressed in terms of the uniaxial break stress in tension, $\sigma_{\mathrm{Tb}}$, and compression, $\sigma_{\mathrm{Cb}}$. The criterion is a linear combination of the octahedral shear and normal stresses. The cone of maximum angle emerges as a special case of the general cone, when $\sigma_{\mathrm{Cb}} \gg \sigma_{\mathrm{Tb}}$

The elliptic paraboloid is a linear combination of the mean normal stress and the square of the octahedral shear stress. It is intuitively appealing because it avoids the physically unlikely angular apex of the cone in the first octant of principal stress space ${ }^{(1)}$. The plane, Eq. (5), again represents the special case where $\sigma_{\mathrm{Cb}} \gg \sigma_{\mathrm{Tb}}$. In small strain theory the plane can be associated with the dilatational strain energy. When dealing with large deformations it is best referred to as the principal octahedral plane.

In the following sections multiaxial failure data obtained on polymeric materials will be reviewed against the background of the failure 


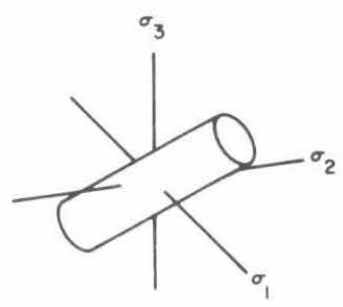

(a)

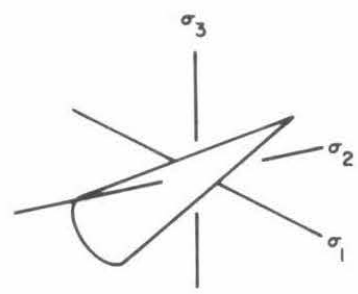

(b)

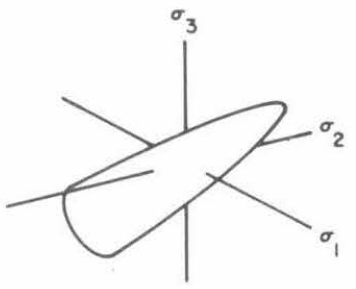

(c)

Fig. 2. Failure Surfaces in Principal Stress Space (Tschoegl (1)). a) Cylinder, b) Cone, c) Paraboloid.

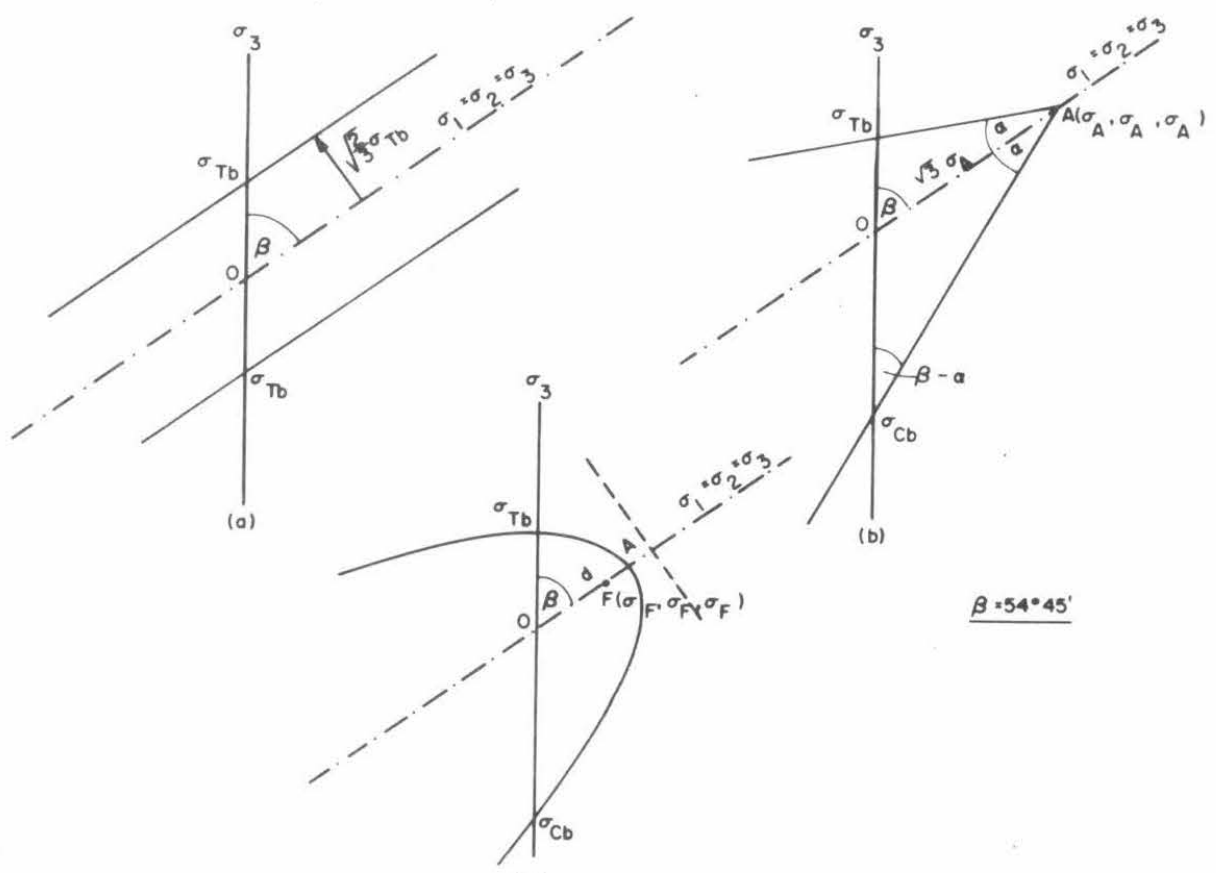

(c)

Fig. 3. Intersection of Failure Surfaces with $\sigma_{1}=\sigma_{2}$ Plane (Tschoegl ${ }^{(1)}$ ). a) Cylinder, b) Cone, c) Paraboloid. 
surfaces discussed above. A more extensive discussion of the theory of failure surfaces was presented in the previous annual report ${ }^{(1)}$, from which Fig. 2, showing the cylindrical, conical, and paraboloid surfaces in principal stress space, and Fig. 3, their intersections with the $\sigma_{1}=\sigma_{2}$ plane were reproduced.

\section{1. Plastics}

The effect of biaxial stresses on the deformation and failure of poly(methylmethacrylate) (Rohm and Haas Plexiglas G) has been studied by Thorkildsen and Olszewski ${ }^{(6)}$ by simultaneously stretching a thin walled tube in the axial direction and applying internal pressure. The experimental results are shown in Fig. 4. The fracture stress could be described by the octahedral shear stress (Huber-Hencky-Mises) theory. The figure also shows, for comparison, the failure area corresponding to the maximum stress (Rankine) theory, which is indistinguishable in the first quadrant from the maximum shear (Tresca-Guest) theory.

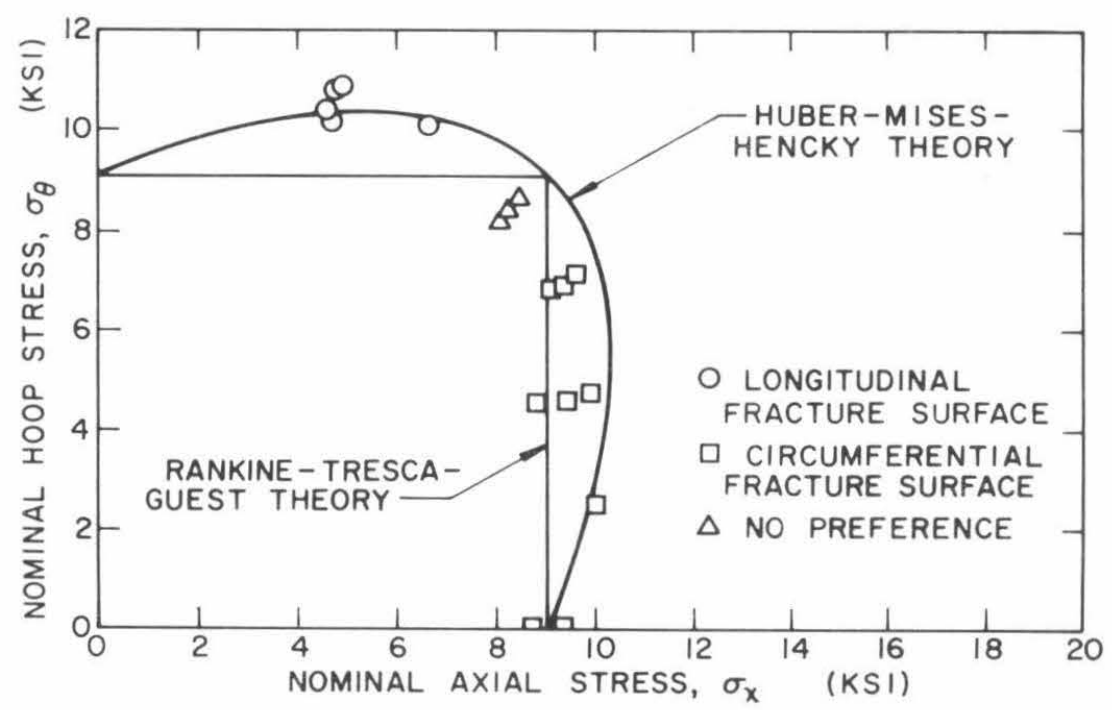

Fig. 4. Fracture Stress for Poly(methylmethacrylate) (Thorkildsen and Olszewski ${ }^{(6)}$ ). 
Biaxial failure behavior on a polyamide (nylon), (7) and a cast acrylic $(\text { Cadco composition } \mathrm{HC}-206)^{(8)}$ were investigated by Ely by applying an axial load and internal pressure to thin walled cylindrical specimen. Ely devised a simple and ingenious piston-and-cylinder arrangement in which the axial load as well as internal pressure in the specimen can be controlled by the uniaxial load alone. For both nylon and acrylic tubes, the cylindrical criterion was appropriate when correlations were restricted to small effective strains (1\%). However, for large effective strains (2\%), and for fracture, the paraboloid was found to be more suitable, especially when the behavior in both the Ist and IVth quadrant of the principal stress plane were considered. The results are shown in Figs, 5 and 6 . The experimental

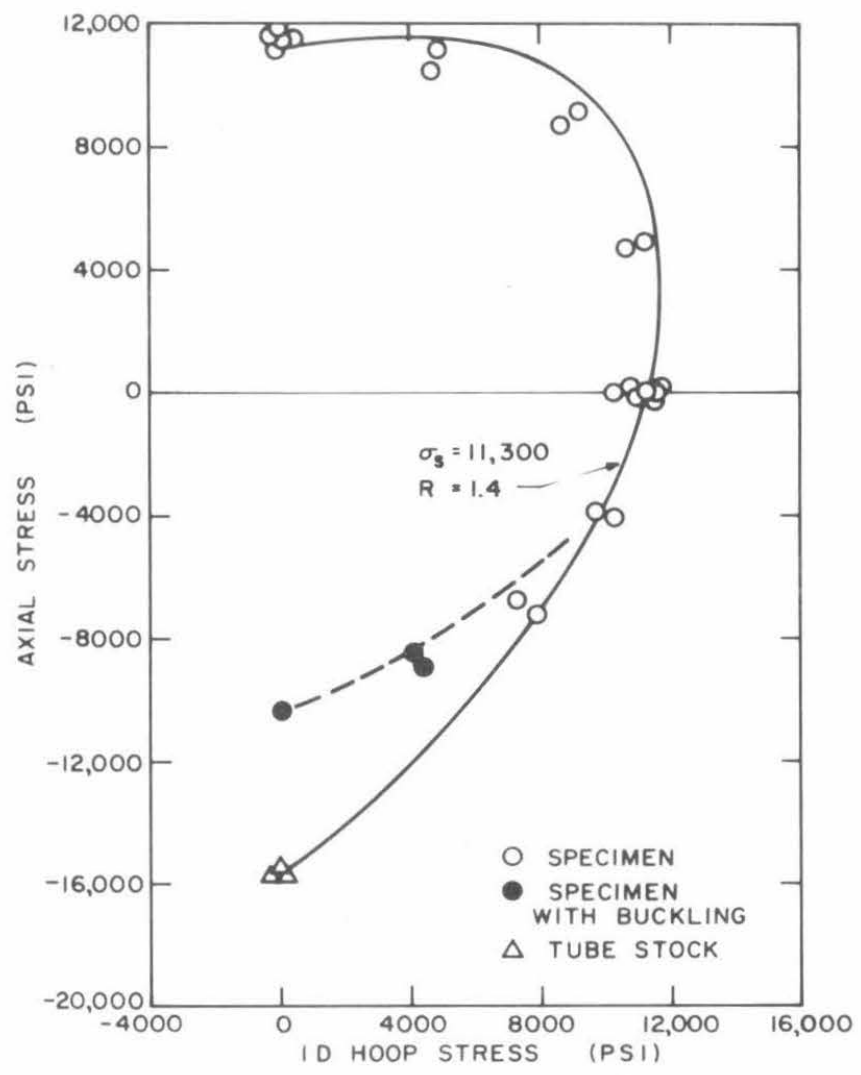

Fig. 5. Biaxial Fracture of Nylon (Ely $\left.{ }^{(7)}\right)$. 


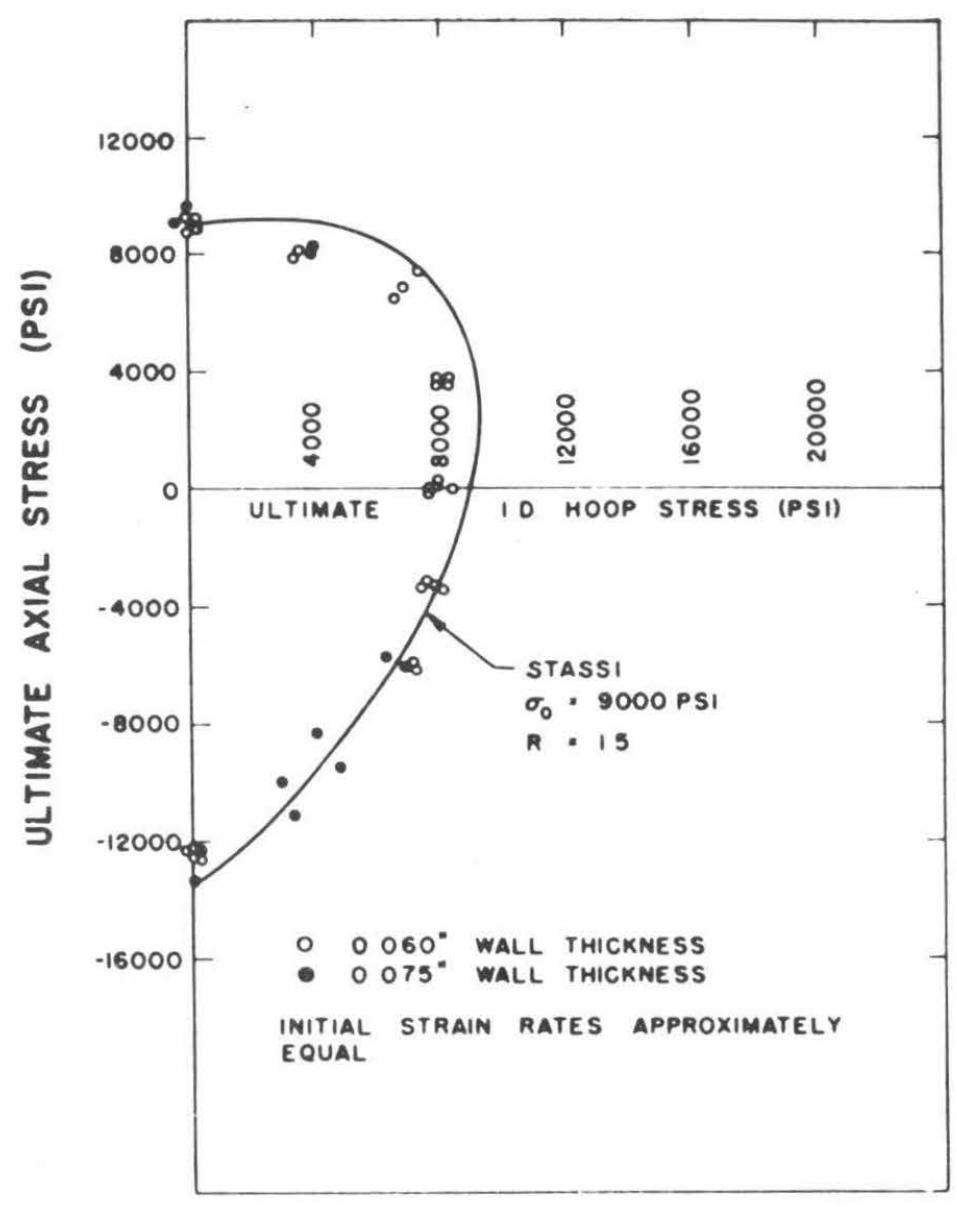

Fig. 6. Biaxial Fracture of Acrylic (Ely $\left.{ }^{(8)}\right)$. data agree well with the previous results by Thorkildsen ${ }^{(6)}$ in the first quadrant. The Stassi equation ${ }^{(9)}$ mentioned in Ely's publications $(7,8)$ is identical with Eq. (4) for the paraboloid.

\section{2 Unfilled Elastomers}

Smith and Rinde $\mathrm{e}^{(10)}$ investigated the biaxial failure behavior of an unfilled styrene-butadiene rubber (SBR) imposing an axial load and internal pressure on thin walled cylindrical specimens. They concluded that neither the octahedral shear stress criterion nor the Tresca condition represented 
the data precisely. The deviation from the octahedral shear stress criterion was negligible within the accuracy demanded for engineering application. The axial extension ratio at break was found to be the same in biaxial and uniaxial experiments within the probable experimental error. The results are shown in Fig. 7. Lim ${ }^{(11)}$ investigated the biaxial failure

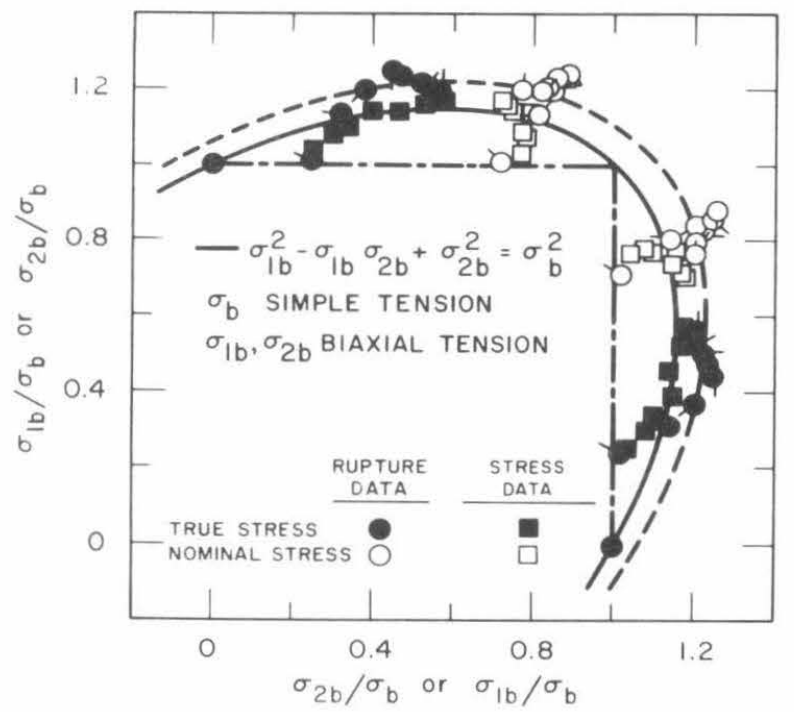

Fig. 7. Biaxial Failure of $\operatorname{SBR}$ (Smith and Rinde ${ }^{(10)}$ ).

behavior of the solid propellant binders, Paracril and Solithane at room temperature. Paracril is a copolymer of butadiene and acrylic acid crosslinked with Epon 828 epoxy resin. The Solithane used here was a polyurethane elastomer similar to GALCIT I (cf. Part IV, Section 1), except that three parts by weight of prepolymer were mixed with four parts of the curing agent, while GALCIT I is a 50-50\% mixture by volume. Failure data were obtained at different stress rates on tubular specimens. The results plotted in true principal stress plane, are shown in Figs. 8 and 9. These data do not conform to any of the failure criteria discussed above. They provide a clear example of the effect of loading rate on the failure criteria. 


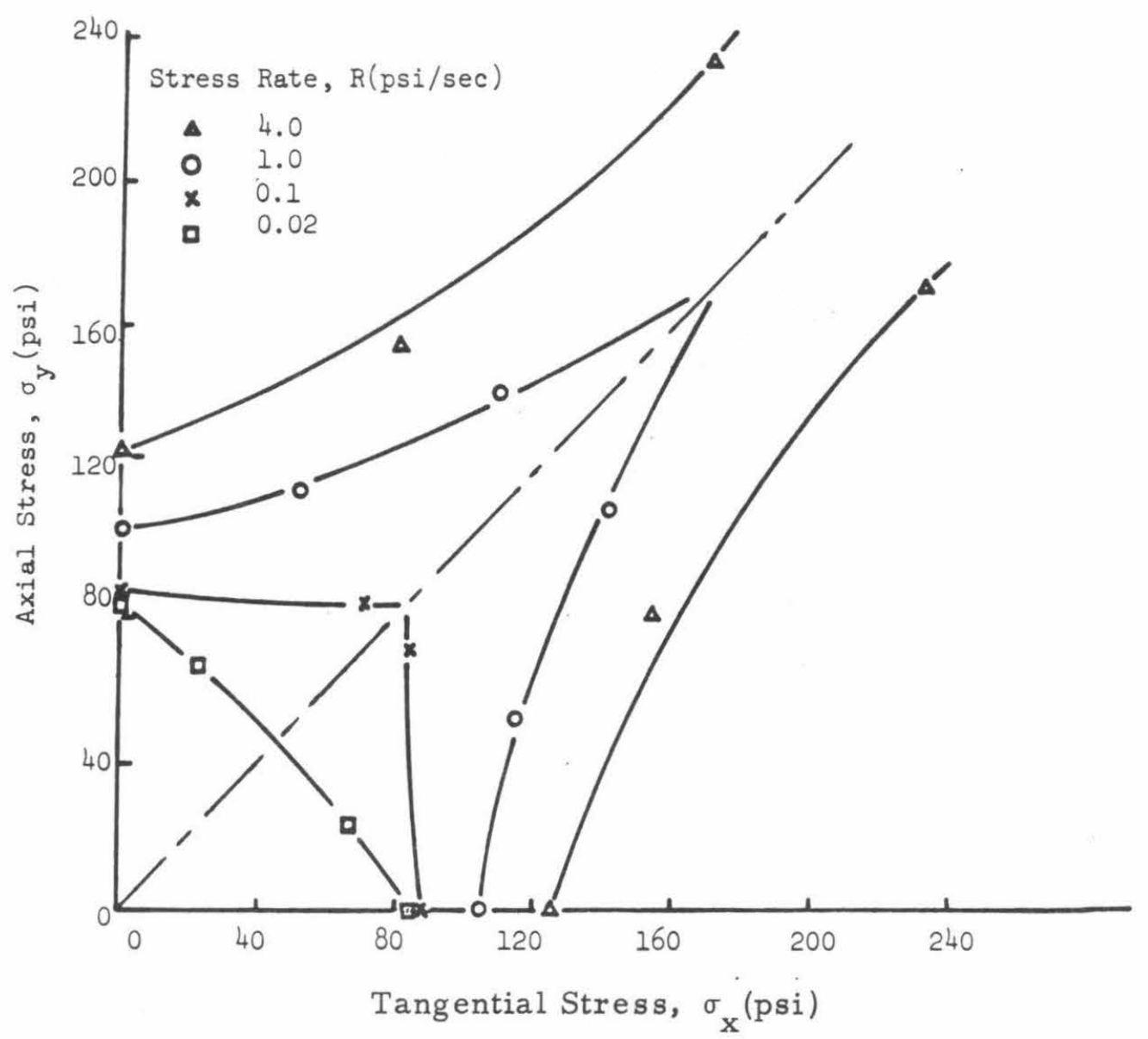

Fig. 8. Biaxial Failure of Paracril (Lim (11)). 


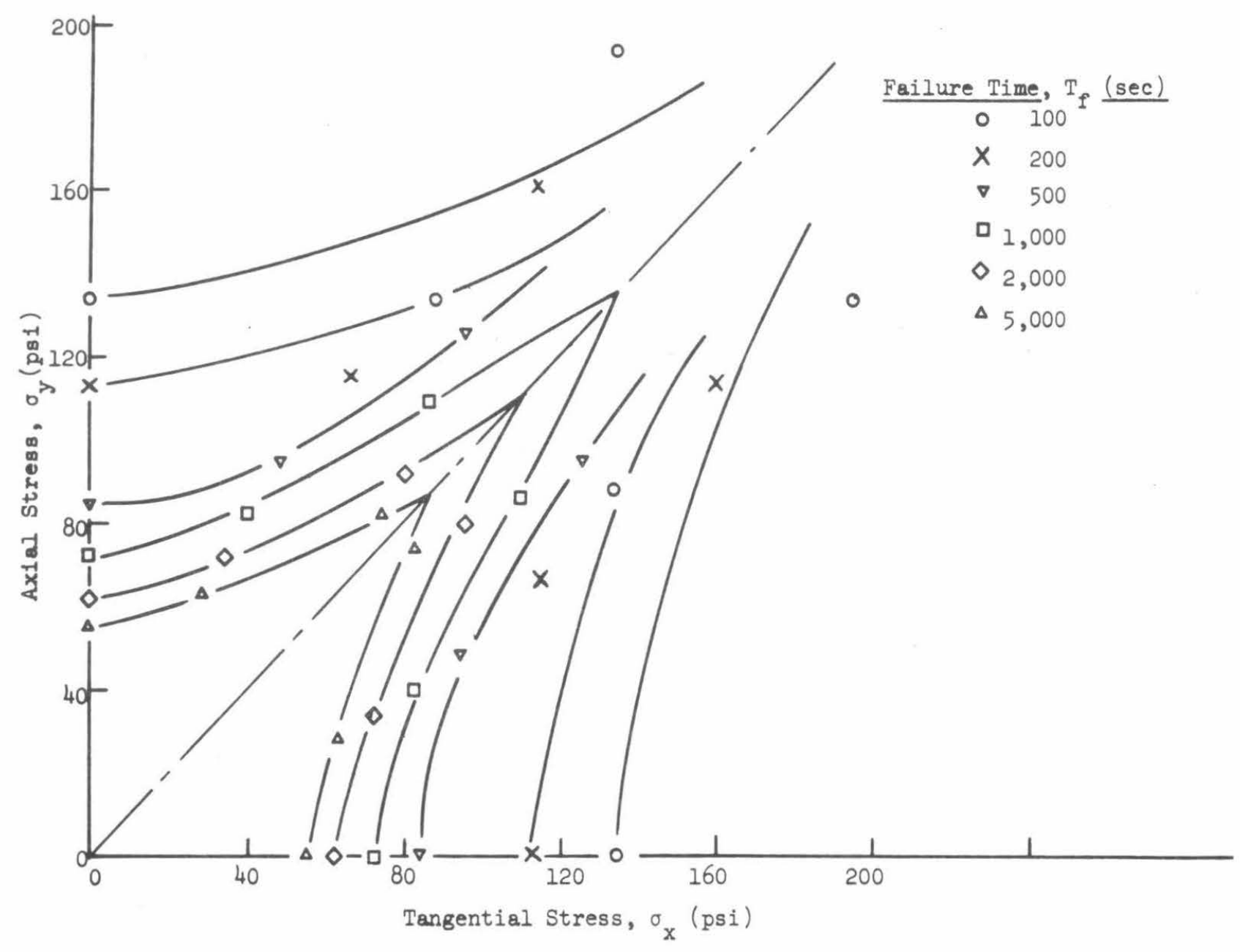

Fig. 9. Biaxial Failure of Solithane $\left(\operatorname{Lim}^{(11)}\right)$. 
The experiments discussed so far all produced failure areas in the principal stress plane. The first attempt to determine a failure surface in the first octant of principal stress space was made by Ko ${ }^{(12 a, 12 b)}$, who investigated the failure surfaces of a polyurethane rubber by means of uniaxial, strip biaxial, and triaxial tension experiments. The failure points in normalized true stress space appear to define a pyramid with apex on the space diagonal as shown in Fig. 10.

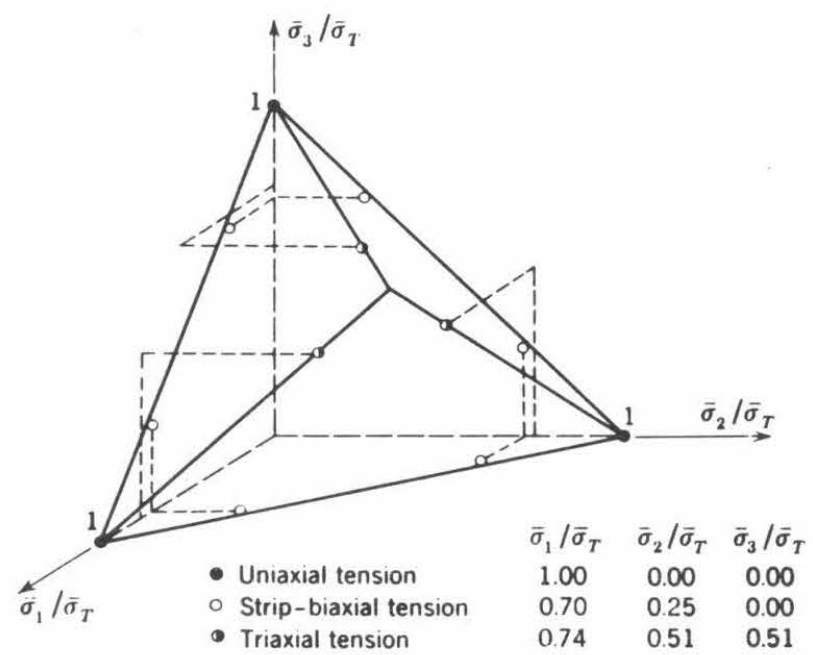

Fig. 10. Failure Surface of Polyurethane Rubber in First Octant of Stress Space $\left(K_{0}^{(12 a, b)}\right)$.

\section{3 Foams}

Using the same experimental approach, Ko and Blatz $(12 a, 13,14)$ also determined the failure surface of a polyurethane foam rubber containing $47 \%$ voids. The failure points in normalized true stress space obtained with this material are shown in Fig. 11. They lie on the principal octahedral plane, conforming to the criterion given by Eq. (5). 


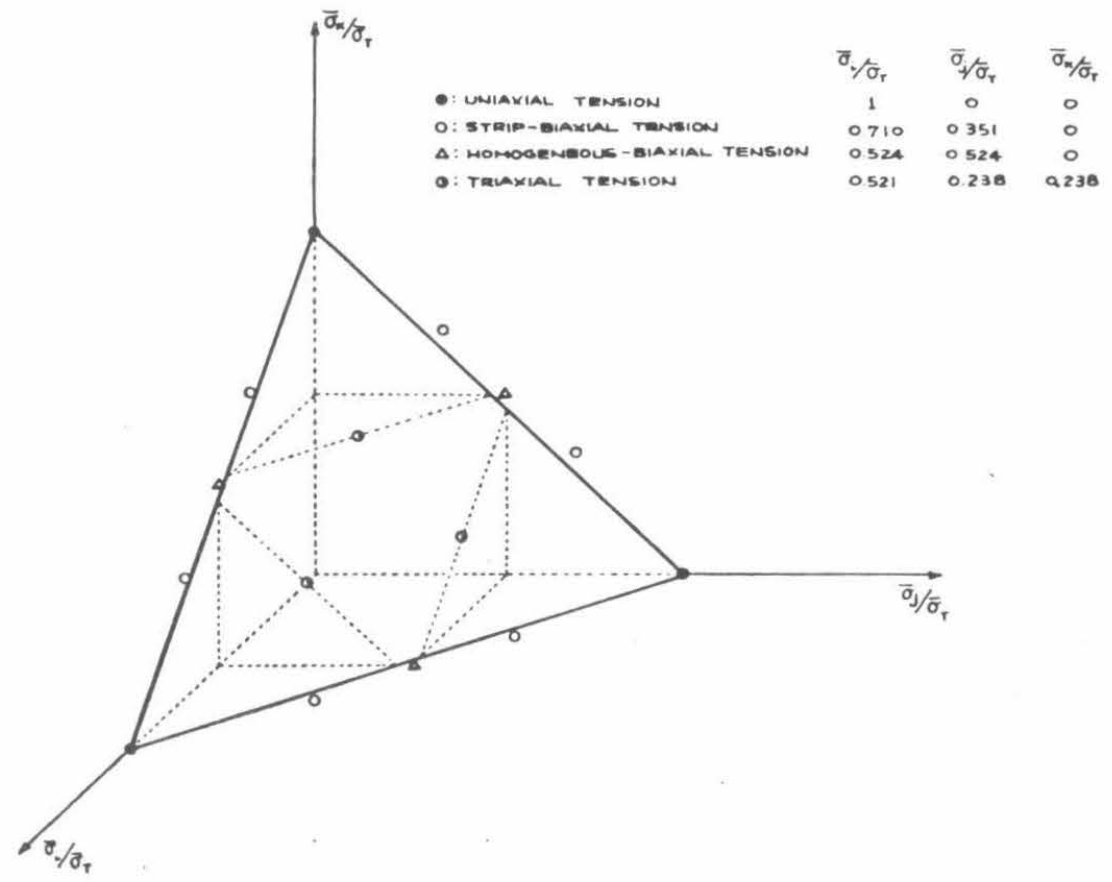

Fig. 11. Failure Surface of Polyurethane Foam Rubber in First Octant of Stress Space (Blatz ${ }^{(14)}$ ).

\subsection{Filled Elastomers and Propellants}

Sharma and Lim ${ }^{(15)}$ examined the failure behavior of filled Hycar, a solid propellant dummy material. Filled Hycar is a copolymer of butadiene and acrylic acid crosslinked with Epon 828 epoxy resin, and filled with $70 \%$ by weight of aluminum spheres of 10 micron diameter. Tests on tubular specimens subjected to uniaxial tension, pure internal pressure, internal pressure combined with tension, pure torsion, and torsion combined with tension, and on solid cylindrical specimens in compression, resulted in the biaxial failure behavior shown in Fig. 12. The failure data in the fourth quadrant are not regarded as representative for this material because 


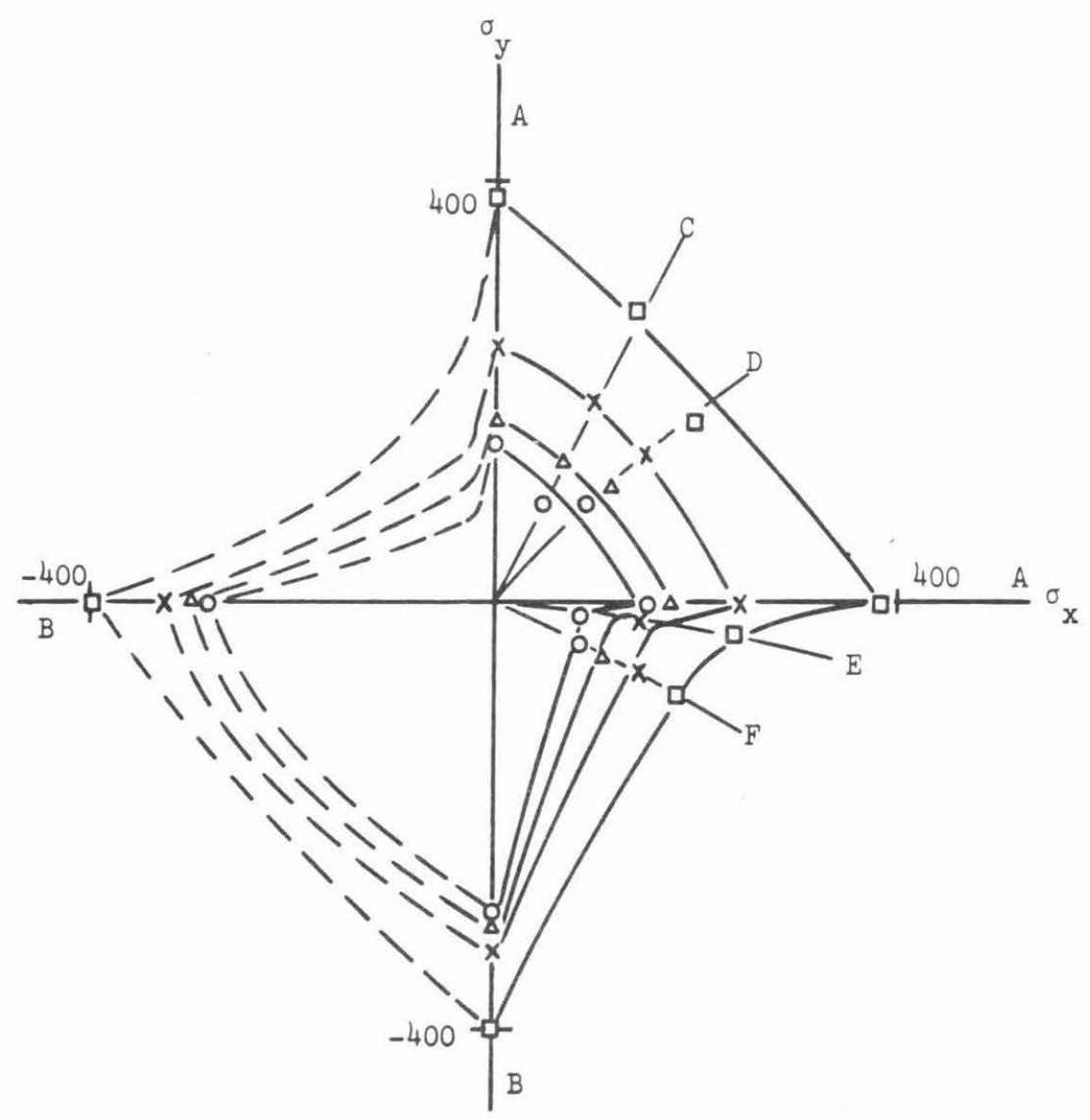

$\lambda=$ Extension ratio rate

$0-0.001 \mathrm{in} / \mathrm{in} / \mathrm{sec}$

$\Delta-0.001 \quad "$

$x-0.01 \quad "$

口- 0.1 "

A - Tension data

B - Compression data

C - Pressure data

D - Tension pressure data

E - Tension torsion data

F - Torsion data

$\sigma_{x}, \sigma_{y}-$ Principal stresses

Fig. 12. Biaxial Failure Behavior of Filled Hycar (Sharma and Lim ${ }^{(15)}$ ). 
of buckling and failure outside of the gage section. In the first quadrant, however, the failure behavior is not too different from that of the polyurethane foam rubber, and from that of a hydrocarbon propellant reported by Jones and Kruse ${ }^{(16)}$. The latter authors combined uniaxial, strip-biaxial, and triaxial extension tests to define a failure surface in stress space which corresponded to the principal octahedral plane. The results are shown in Fig. 13. Extension of the data into the compressive octants to form a

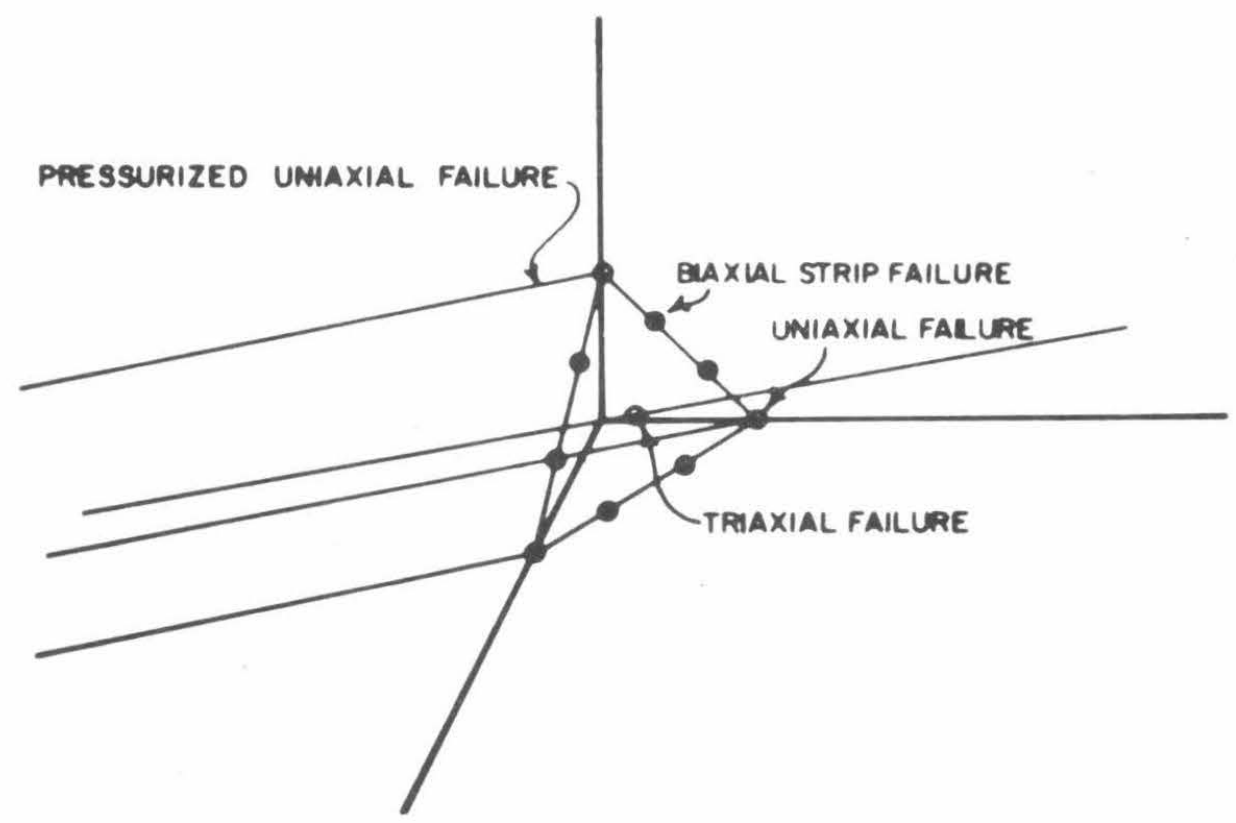

Fig. 13. Failure Surface of a Hydrocarbon Propellant in First Octant of Stress Space (Jones and Kruse ${ }^{(16)}$ ). prismatic failure surface is based on inference from uniaxial tension data obtained under superposed hydrostatic pressure. The tests indicated that the failure stress of this propellant was independent of the hydrostatic pressure. The break stress of another, more compressible propellant, however, showed some dependence on the pressure. The results are shown in Fig. 14. 


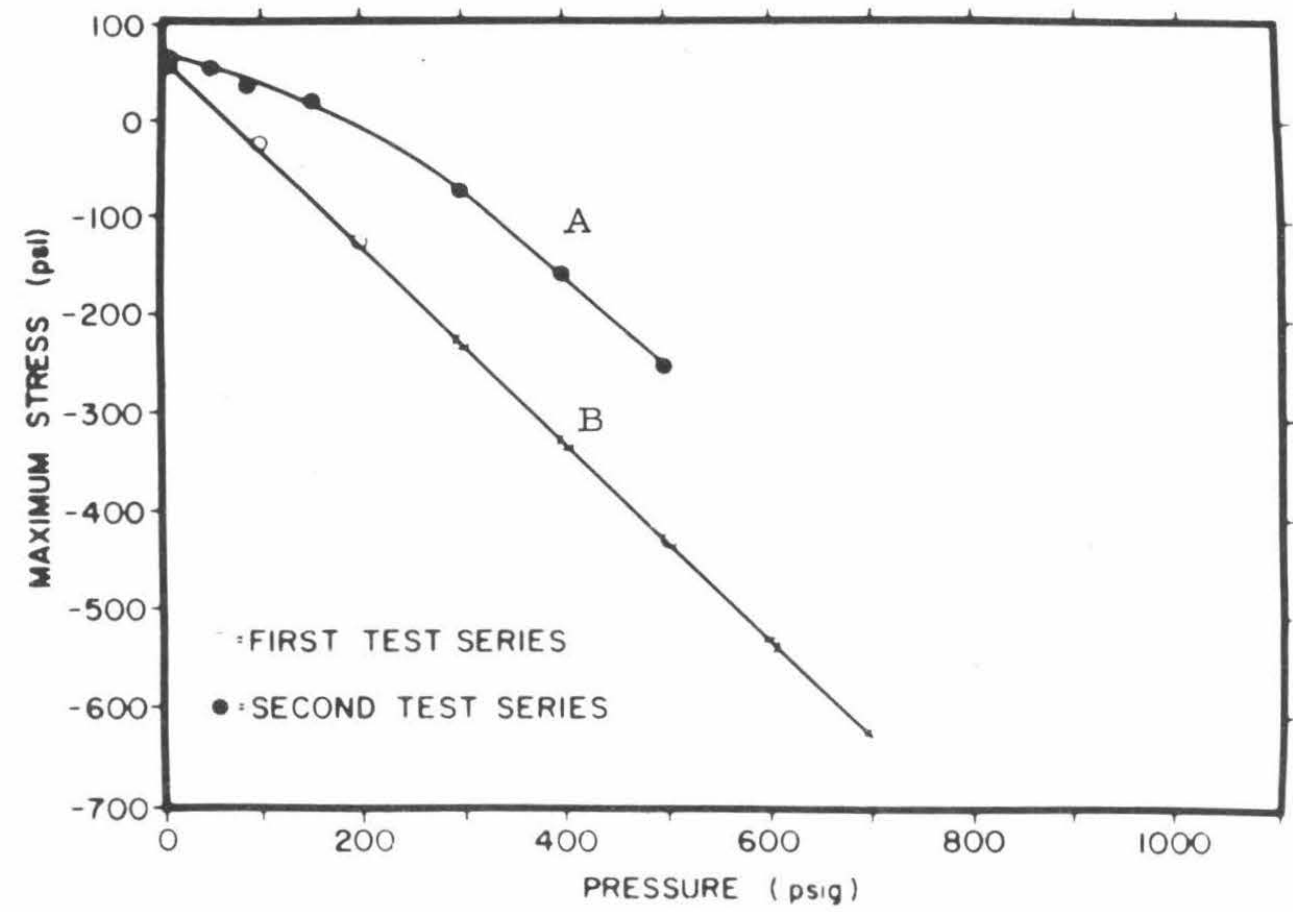

Fig. 14. Total Uniaxial Stress at Break of a Compressible (A) and an Incompressible (B) Hydrocarbon Propellant under Superposed Hydrostatic Pressure (Jones and Kruse (Ref. 16)).

Jones and Knauss ${ }^{(17)}$ investigated the failure surface of poly(butadiene-acrylonitrile) (PBAN) propellants with volumetric loadings of 70 and $80 \%$. Seven tests, representing a multiplicity of locations in threedimensional stress space, were used to construct a three-dimensional representation of the failure surface. The calculations were carried out on the basis of infinitesimal deformation theory.

While pointing out that the data and their interpretation lack the support of a precise stress analysis, the authors observed an approximate agreement with a maximum principal stress criterion of failure in the first octant of stress space. The data for the other octants appeared to support a linear internal friction criterion (Coulomb-Duquet-Navier theory). The 
tensile failure stress under superposed hydrostatic pressure was independent of the pressure up to 1000 psig.

\subsection{Conclusions}

No clear-cut picture emerges from the experimental evidence presented in the preceding sections. The failure behavior of some materials appear to obey one or the other of the simple criteria embodied in Eqs. (1) through (5). Others, however, evince totally different behavior (cf. e.g. Figs. 8 and 9). Where simple behavior is observed, there is no clue why any one of the criteria is obeyed rather than another. If any general conclusion may be drawn, it is that the behavior of the plastics and unfilled elastomers appears to be strengthened in a biaxial stress field, while that of foams and filled elastomers appears weakened.

There is evident need for much more experimental investigation if a fuller understanding of the failure behavior of polymeric materials under multiaxial loads is to be attained. Data are particularly needed in the compressive octants of principal stress space. Improvements in methodology also seem to be desirable so that the experimental findings can be interpreted with a greater degree of confidence.

\section{Tensile Strength and Stress-Strain Behavior of Swollen Elastomers} under Superposed Hydrostatic Pressure

This section deals with our attempt to generate failure data on filled and unfilled rubbers in the compressive octants of principal stress space by carrying out uniaxial, (and, hopefully later, biaxial) tension tests under superposed hydrostatic pressure. Section 3.1 describes the apparatus. 
Section 3.2 deals with the calibration and experimental procedure. The tests are described in Section 3.3, and the results are discussed in Sections 3. 4, 3.5, and 3.6.

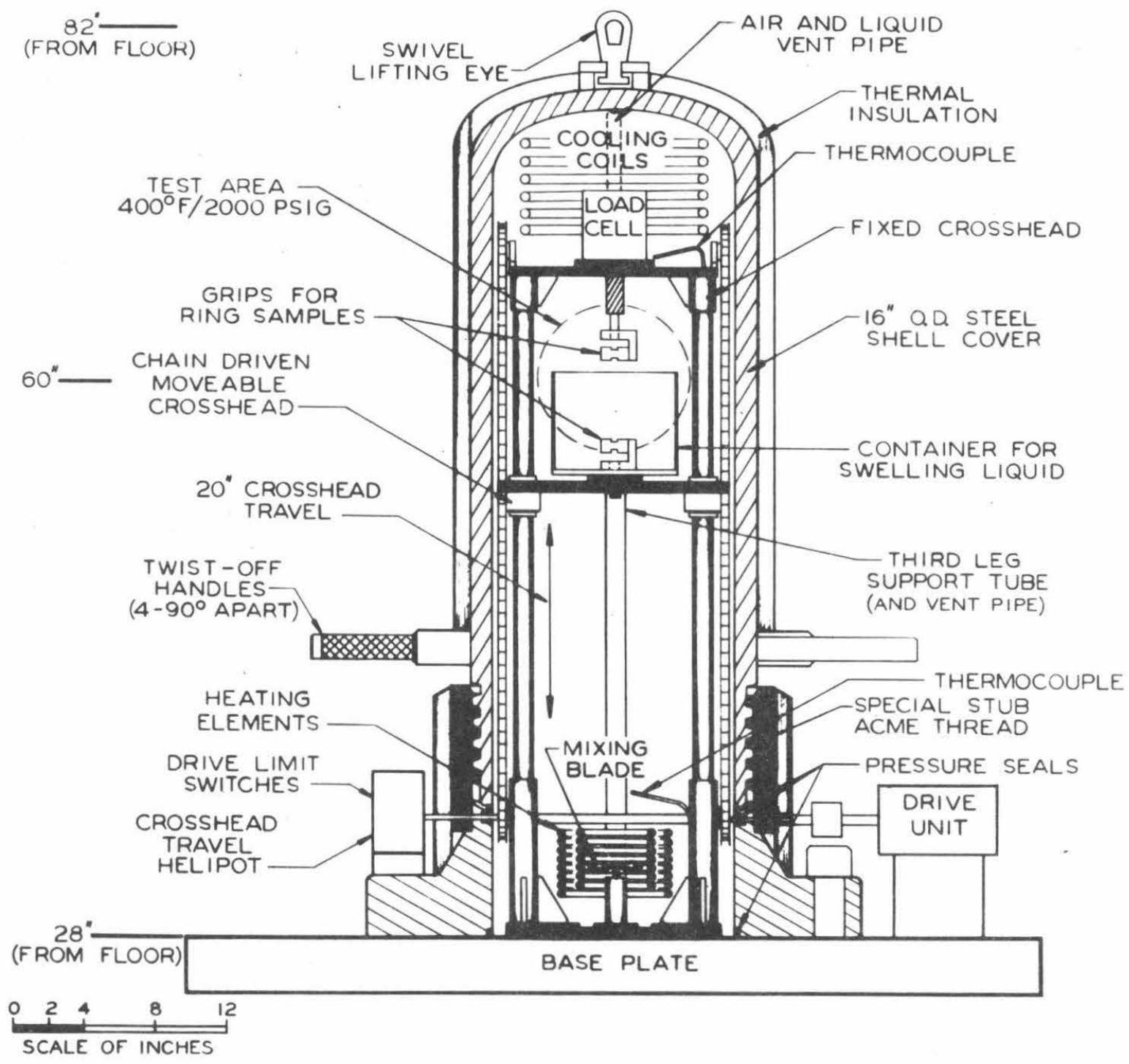

Fig. 15. Schematic of the Pressurized Tensile Tester. 


\section{1 Description of the Apparatus}

Figure 15 shows an up-to-date schematic of the apparatus which was constructed to carry out tensile tests on rubber rings under superposed hydrostatic pressure up to 2000 psig. A detailed description of the pressurized tensile tester was given previously ${ }^{(1)}$. The apparatus was designed in the Central Engineering Shop of the California Institute of Technology. The base plate, shell, and drive mechanism was manufactured by the L \& M Foundry Company, Los Angeles, to the Shop's specifications, and were assembled with the drive unit, displacement transducer, the limit switches, heating and cooling coils, and the drive motor in the Shop. The unit was then subjected to careful pressure tests.

In the first test the unit was brought to a pressure of 3400 psig in increments of a few hundred psig. Several small leaks appeared at 100 psig which were easily eliminated by tightening. The drive unit was operated at 2000 and 3000 psig. No further leaks appeared. In the second test it was ascertained that the burst diaphragm ruptures, as intended, at 2200 psig $10 \%$ above the maximum working pressure of 2000 psig. Finally, in the third test the unit was left at 2000 psig overnight. The total pressure drop over a period of eighteen hours was $10 \mathrm{psig}$. This drop is probably due to temperature differences between the late afternoon and the early morning hours. No traces of any leak were found outside of the unit. After successful completion of the tests the unit was removed from the Central Engineering Shop and installed in the Spalding Laboratory where the hydraulic and electronic systems were added.

The piping schematic of the pressurized tensile tester is shown in Fig. 16. The hydraulic system consists of the pressurized tensile tester, 


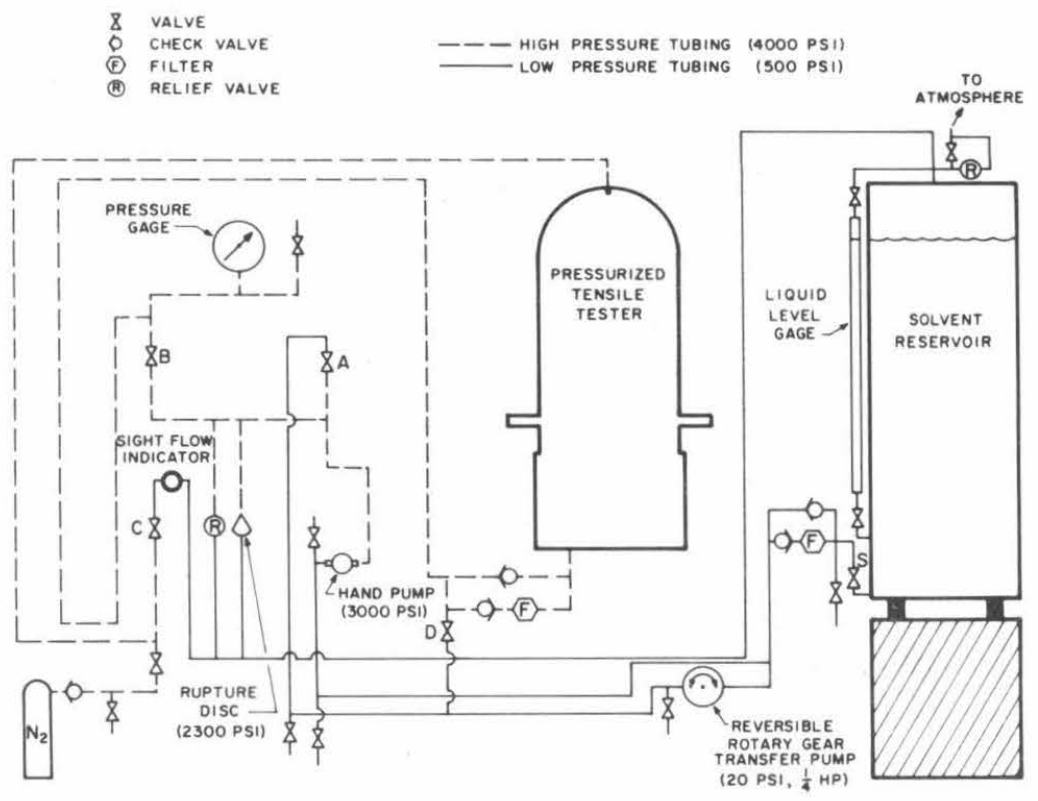

Fig. 16. Piping Schematic for the Pressurized Tensile Tester.

the solvent reservoir, transfer pumps, control panel, and nitrogen pressure back-up system. A set of filters and check valves was added to avoid contamination of the lines and a bypass was mounted between the storage tank and the pressure vessel to speed up the transfer of liquid. The solvent reservoir is a 40 gallon capacity A. O. Smith Corporation, Model KGA40751 gas fired household water heater and storage tank. It is provided with a liquid level site gauge and an atmospheric vent. The transfer pump is a Lobee Pump and Machining Co. Model L-2 SST-SG reversible rotary gear pump driven by a quarter-hourse power Westinghouse AC motor. The control panel consists of a $4^{\prime} \times 7^{\prime} \times 3 / 16^{\prime \prime}$ aluminum plate. It contains a calibrated Bourdon dial type pressure gauge (Marsh Instrument Co. 20 psi subdivision, 5000 psig maximum pressure), the control and safety valves, the rupture disk, the sight flow indicator, and a hand pump 
for pressurization. The hand pump is a Sprague Engineering Co. single action hand pump. The rupture disk is a 1/2-inch STD type Continental Disk Corporation device with Inconel disk and SA-10 safety crown. The rupture pressure at $72^{\circ} \mathrm{F}$ is 2395 psig.

The nitrogen pressure back-up system is also connected to the control panel. Its purpose is to ensure proper maintenance of the pressure level and to serve for pressurization in case of a breakdown of the hand pump which had to be removed several times for repairs.

The force exerted on the specimen is measured by a $100 \mathrm{lb}$. capacity Baldwin-Lima-Hamilton Model U-3Gl load cell instead of the 50 lb. load cell reported earlier. The cell was modified for immersions in solvents. The outer housing was removed to ensure easy flow of liquid. The potting material was also removed since it did not stand up to the solvent action of the pressurizing liquid. All lead wires were replaced by Teflon coated wires. A Daytronic Model 300 D Preamplifier with a Model 93 Input Module with Zero Suppression serves as the power supply for the load cell. The output of the load cell is recorded on a Moseley Autograf Model 2FRA $\mathrm{X}-\mathrm{Y}_{1}-\mathrm{Y}_{2}$ two pen recorder.

The displacement of the crosshead is monitored by a Beckman Instrument Co. Model A30K ten-turn helipot. The crosshead is driven by a Bodine Electric Co. Model NHS-12RG 1/50 H. P. d. c. shunt motor with a Minarik Electric Co. Model SH-14 speed controller, through a Boston Gear Co. Model T-118 worm gear speed reducer. The helipot has . $25 \%$ linearity and is driven by the chain drive shaft through a $3: 1$ stepped-up helical gear assembly. The helipot and helical gear assembly is mounted outside of the shell on the base plate opposite the drive unit along with a limit switch 


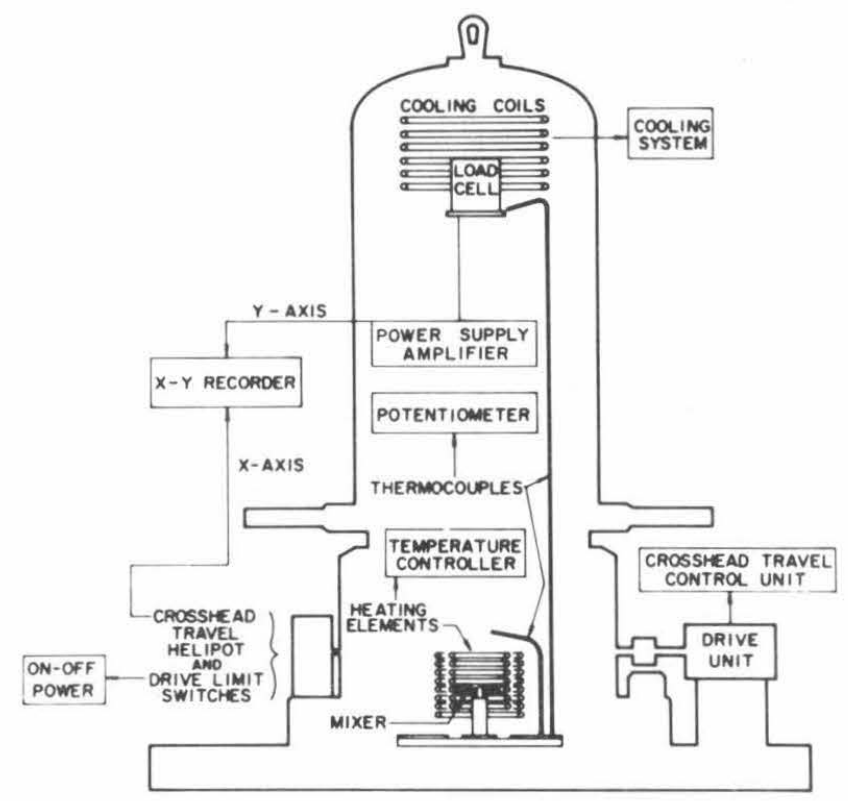

Fig. 17. Electric Schematic for the Pressurized Tensile Tester.

assembly. (See Figs. 15 and 17). Another ten-turn helipot for zero suppression together with the former forms the full bridge circuit. The power supply for the bridge circuit is a Baldwin-Lima-Hamilton Electronics Model 3566 Silicone Solid State DC Power Supply which is continuously variable from 4 to 25 volts.

The instrumentation and electric control units were assembled on a mounting rack. A schematic is shown in Fig. 17. The rack carries the Daytronic Preamplifier, the Moseley Autograf, the power supply and bridge circuit for the displacement monitor, and the speed control for the drive motor. It also carries switching arrangements for the crosshead travel and for the pump, the stirrer, and the heating coils. A Honeywell Model 2732 potentiometer to measure the EMF of the thermocouples, the cold junctions (Dewar flask) for the potentiometers, and a Honeywell-Brown Pyro-Vane thermocontroller are also located in the rack. 


\section{2 Calibration and Experimental Procedure}

The crosshead displacement was calibrated against the pen deflection of the recorder using a dial indicator. The results demonstrated excellent linearity, reproducibility, and no drift. A typical calibration curve is shown in Fig. 18.

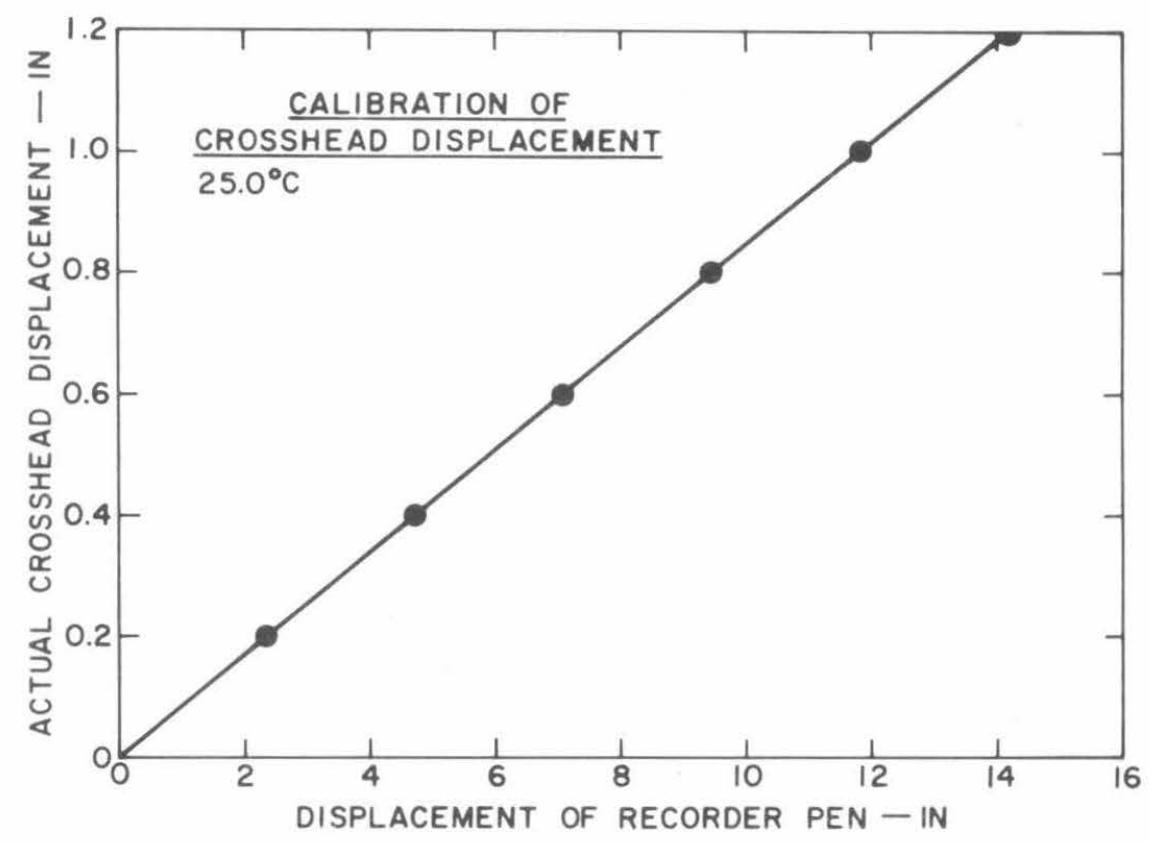

Fig. 18. Calibration of Crosshead Displacement Monitor.

The load cell was calibrated by dead weight calibration in four stages to $2 \mathrm{lb}$. full scale at different pressures. The arrangement for this is shown in Fig. 19. Four 1/2 1b. weights were suspended from the load cell by a flexible fishing line. Initially the weights all rest on the movable crosshead and there is no load on the cell. As the crosshead descends, the weights come into action one at a time. The measurements again indicated very good linearity, reproducibility, and no drift. No effect of bending moment could be detected for deflections which were considerable in excess of any anticipated misalignment. 


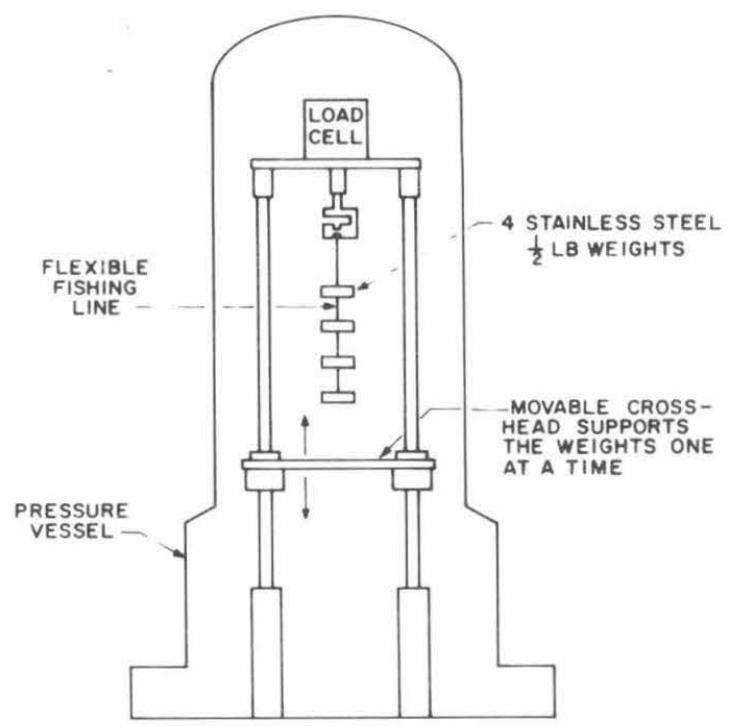

Fig. 19. Experimental Arrangement for Calibration of the Load Cell.

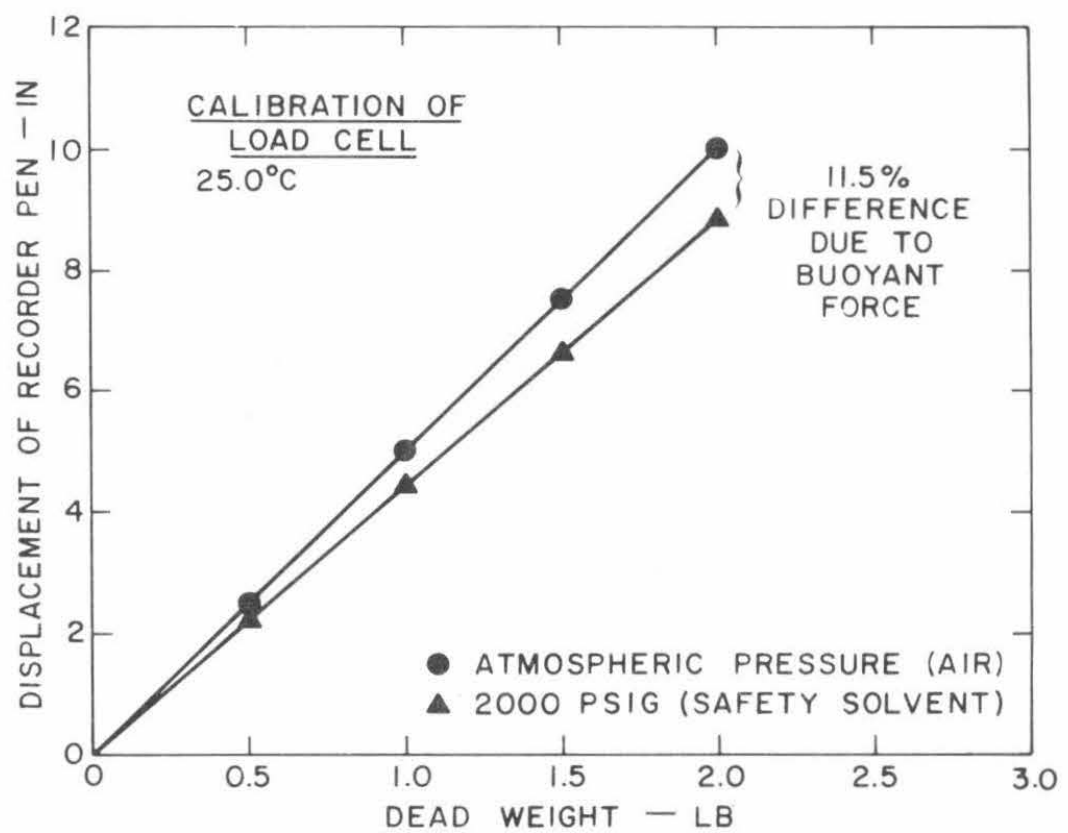

Fig. 20. Calibration of Load Cell Output. 
Figure 20 shows the calibration curves for atmospheric and 2000 psig pressure. The calibration constants differ by $11.5 \%$ and this is exactly due to the buoyant force of the pressurizing liquid on the weight. The density of the solvent was $55.5 \mathrm{lb}$./cu. ft., and that of the No. 303 stainless steel weight was $483 \mathrm{lbs}$./cu. ft. The density ratio indicates precisely $11.5 \%$ weight loss in the solvent. The hydrostatic pressure, therefore, has no effect on the load cell calibration.

The liquid used both as swelling agent and as pressurizing medium was Arnold Laboratory Safety Solvent No. 99, a hydrocarbon solvent containing a small amount of chlorinated hydrocarbons.

The dimensions of the swollen ring specimens were determined with the aid of a traveling microscope. Several measurements were taken across different diameters of the ring and these were averaged. During the measurements the rings were always kept submerged in the solvent. They were then transferred (without leaving the liquid) to a $7^{\prime \prime} \times 8^{\prime \prime} \times 6^{\prime \prime}$ high aluminum box fixed to the movable crosshead and filled with the same solvent. The box has Perspex walls for visibility. The lower pin for stretching ring-shaped specimens is mounted inside the box. The upper pin is connected to the load cell. The pins consisted of Teflon cylinders press-fitted onto stainless steel studs. After the rings were attached to the stretching pins, the shell was lowered into position and closed tightly. Solvent was then pumped from the reservoir tank to the pressure vessel. When the pressure vessel was filled it was pressurized to the desired value by means of either the hand pump or the nitrogen pressure back-up system. A little time was allowed for pressure and temperature equilibration before the test was begun. 


\subsection{Uniaxial Extension Tests on Rubbers under Superposed Hydrostatic}

\section{Pressure}

Two elastomers were used in these tests. The first was a styrenebutadiene rubber (SBR) prepared according to the following formulae:

$\begin{array}{lr}\text { Phillips SBR } 1500 & 100.00 \text { parts } \\ \text { Zinc Oxide } & 5.00 \text { parts } \\ \text { Sulfur } & 2.25 \text { parts } \\ \text { MBTS (benzothiazyl disulfide) } & 2.25 \text { parts } \\ \text { Stearic Acid } & 1.50 \text { parts }\end{array}$

The rubber was cured for one hour at $320^{\circ} \mathrm{F}$ in a mold at 1500 psig pressure.

For the second elastomer we used the interim standard, GALCIT I, which has been described in detail in several earlier reports and in Part IV of this report. GALCIT I is a highly crosslinked polyurethane rubber with particularly high strain optical coefficient.

The tests were made at room temperature at a crosshead speed of 1.45 inches per minute under hydrostatic pressures of $0,500,1000$, 1500 , and 2000 psig. Five identical tests were run for each pressure. The swollen SBR rings had outer diameters of 2.10 inches and inner diameters of 1.85 inches. The outer and inner diameters of the swollen GA LCIT I rings were 1.60 and 1.41 inches respectively.

The linear swelling ratios (measured with the traveling microscope) in the solvent No. 99 were 1.70 for the SBR and 1.28 for GALCIT I. The crosslink density calculated from

$$
\nu_{\mathrm{e}}=\mathrm{G} \alpha / \mathrm{RT}
$$


(where $\alpha$ is the linear swelling ratio) were $114 \times 10^{-6}$ moles per $\mathrm{cm}^{3}$ for the SBR, and $520 \times 10^{-6}$ moles per $\mathrm{cm}^{3}$ for GALCIT I using, for $G$, the average modulus values of 24 psi for SBR, and 146 psi for GALCIT I as discussed in Section 3. 4.

The measured values of the modulus and the stress at break were reduced from the actual test temperature to $25^{\circ} \mathrm{C}$ using the kinetic theory correction. Since the tests were carried out at room temperature the correction was minor. No correction was made for the effect of temperature on the specimen dimensions as this would have been negligible.

The average strain in the rings was calculated by the equation of Smith as detailed in the previous report ${ }^{(1)}$. The data were extrapolated to calculate the tensile strength based on the inner rather than the average circumference of the ring ${ }^{(1)}$.

The three principal stress were obtained from

$$
\begin{aligned}
& \sigma_{1}=\sigma_{2}=-p \\
& \sigma_{3}=-p+\sigma_{T}
\end{aligned}
$$

where $p$ is the hydrostatic pressure and $\sigma_{T}$ is the tensile stress calculated from the force measured by the load cell.

\section{4 Effect of Hydrostatic Pressure on Modulus}

The effect of the hydrostatic pressure on the reduced moduli is shown in Fig. 21 for the SBR, and in Fig. 22 for GALCIT I. The correlation 


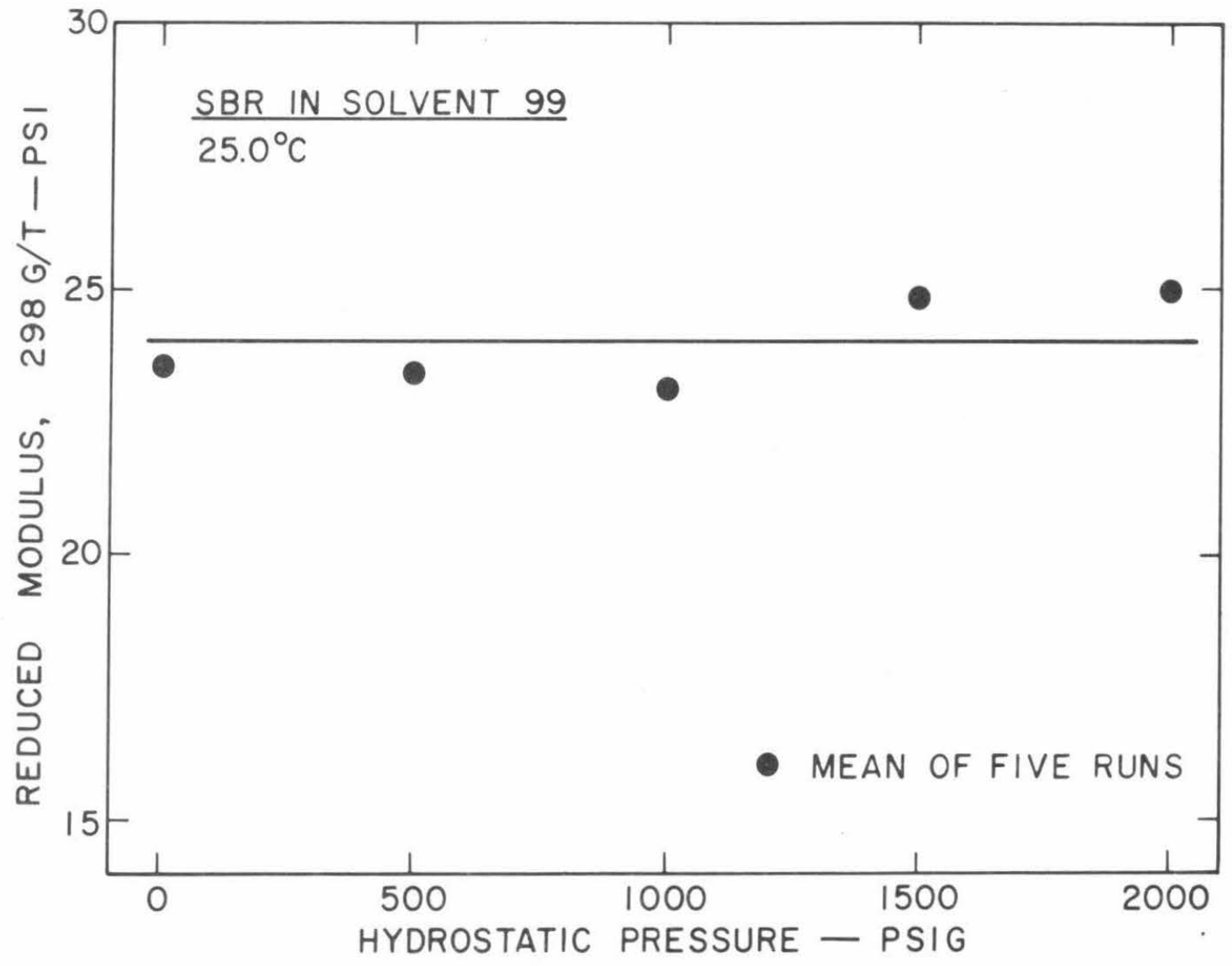

Fig. 21. Effect of Hydrostatic Pressure on the Modulus of Swollen SBR. 


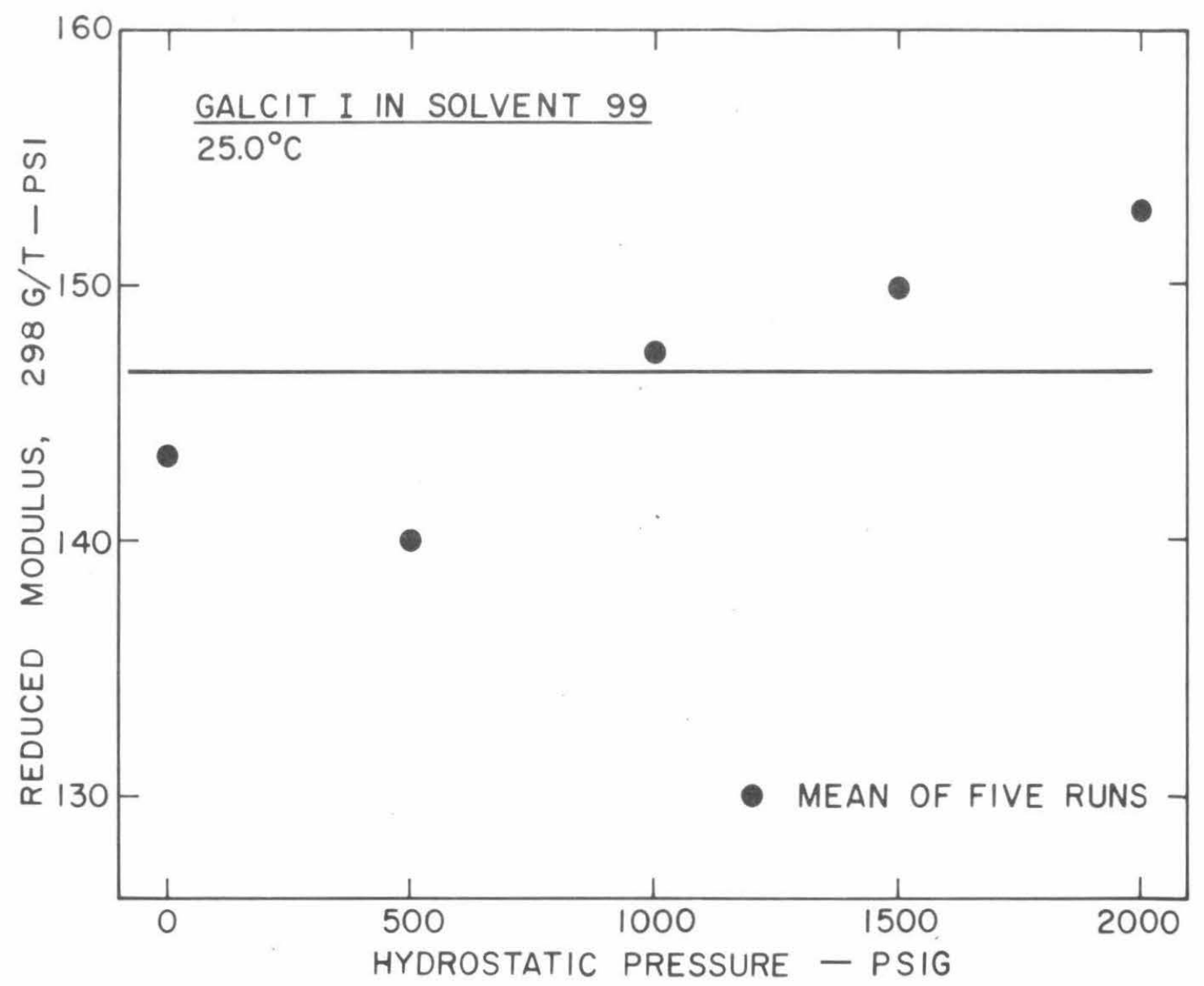

Fig. 22. Effect of Hydrostatic Pressure on the Modulus of Swollen GA LCIT I. 
coefficient between the reduced moduli and the hydrostatic pressure was 0.28 for the SBR, and 0.62 for GALCIT I. The former is not significant, but the latter is significant at the 5\% level of significance. A careful examination of the data showed, however, that this trend in the GALCIT I modulus with pressure is in all probability due to experimental error in the determination of the cross-sectional area. The trend was therefore disregarded and it is concluded that the hydrostatic pressure had no effect on the reduced modulus of either swollen material.

The statistical information on the modulus determinations is assembled in Tables I and II. The mean modulus at $25^{\circ} \mathrm{C}$ for the swollen SBR, calculated from all 25 measurements, was 24 psi with a standard deviation of $2.2 \mathrm{psi}$, and a coefficient of variation of $9.1 \%$. For swollen GALCIT I the values were $146 \mathrm{psi}, 6.7 \mathrm{psi}$, and $4.6 \%$, respectively. The GALCIT I moduli, therefore, showed considerable less scatter than the SBR moduli. An explanation for the greater scatter of the SBR moduli may well be found in the difference in mode of preparation of the two samples. The SBR specimens were compression molded, and thus crosslinked in the mold, while the GALCIT I specimens were cast, and thus crosslinked during the polymerization. This difference in the crosslinking procedure could conceivably lead to greater variation in the local crosslink density over the sheet from which the SBR rings were cut.

\section{5 Effect of Hydrostatic Pressure on the Ultimate Properties}

Figure 23 shows a plot of the (neohookean) tensile strain at break, $\lambda_{b}-\lambda_{b}{ }^{-2}$, against the hydrostatic pressure. No effect of the hydrostatic pressure can be seen. The statistical information is again assembled in 
TABLE I

\section{Statistics for Pressurized Tensile Tests}

on Swollen SBR Rings

(Room Temperature)

Mean $\begin{gathered}\text { Standard } \\ \text { Deviation } \\ \begin{array}{c}\text { Coefficient } \\ \text { of Variation } \\ (\%)\end{array}\end{gathered}$

Total (all pressures, $N=25$ )

$298 \sigma_{b} / T(p s i)$

$\lambda_{b}-\lambda_{b}-2$

$298 \mathrm{G} / \mathrm{T}$ (psi)

24. 31

0.999

4. 92

20. 2

0.191

23. 95

2. 17

9. 1

$$
500 \operatorname{psig}(\mathrm{N}=5)
$$

$298 \sigma_{b} / T(p s i)$

$\lambda_{b}-\lambda_{b}-2$

25.76

1.076

$298 \mathrm{G} / \mathrm{T}$ (psi)

23. 42

$1500 \mathrm{psig}(\mathrm{N}=5)$

$298 \sigma_{b} / T$ (psi)

$\lambda_{b}-\lambda_{b}-2$

27. 54

1. 080

24.82
1. 14

5.87

0.170

1. 40

6.32

24. 5

26.3

4. 9

21. 3

15. 7

5. 6

24.89

23. 94

0.956

0.189

1. 96

4. 149

18. 4

0.977

0.198

20.3

23. 12

3. 50

15. 2

2000 psig $(N=5)$
4. 00
16. 7

9. 7

7.9 
TABLE II

Statistics for Pressurized Tensile Tests

on Swollen GALCIT I Ring s

(Room Temperature)

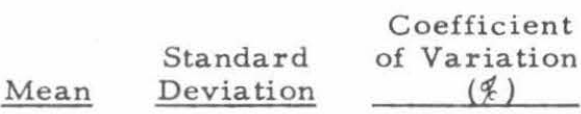

\begin{tabular}{|c|c|c|c|c|c|c|}
\hline & Mean & $\begin{array}{l}\text { Standard } \\
\text { Deviation }\end{array}$ & $\begin{array}{c}\text { Coefficient } \\
\text { of Variation } \\
(\notin) \\
\end{array}$ & Mean & $\begin{array}{l}\text { Standard } \\
\text { Deviation }\end{array}$ & $\begin{array}{c}\text { Coefficient } \\
\text { of Variation } \\
(\$)\end{array}$ \\
\hline & \multicolumn{3}{|c|}{ Total (all pressures, $\mathrm{N}=25$ ) } & \multicolumn{3}{|c|}{$0 \operatorname{psig}(N=5)$} \\
\hline $298 \sigma_{\mathrm{b}} / \mathrm{T}(\mathrm{psi})$ & 92.20 & 18.83 & 20.4 & 88.16 & 19.28 & 21.9 \\
\hline$\lambda_{b}-\lambda_{b}-2$ & 0.617 & 0.118 & 19.1 & 0.601 & 0.109 & 13.1 \\
\hline 298 G T (psi) & 146.64 & 6.70 & 4.6 & 143.25 & 5.95 & 4. 2 \\
\hline & \multicolumn{3}{|c|}{$500 \operatorname{psig}(N=5)$} & \multicolumn{3}{|c|}{$1000 \mathrm{psig}(\mathrm{N}=5)$} \\
\hline $298 \sigma_{\mathrm{b}} / \mathrm{T}(\mathrm{psi})$ & 83.60 & 17.26 & 20.7 & 84.31 & 22.73 & 27.0 \\
\hline$\lambda_{b}-\lambda_{b}-2$ & 0.585 & 0.121 & 20.7 & 0.562 & 0.153 & 27.2 \\
\hline 298 G/T (psi) & 139.98 & 8. 34 & 6.0 & 147.31 & 3.12 & 2. 1 \\
\hline & \multicolumn{3}{|c|}{$1500 \operatorname{psig}(N=5)$} & \multicolumn{3}{|c|}{$2000 \operatorname{psig}(N=5)$} \\
\hline $298 \sigma_{\mathrm{b}} / \mathrm{T}$ (psi) & 103.35 & 13.67 & 13.2 & 101.57 & 172.65 & 17.0 \\
\hline$\lambda_{b}-\lambda_{b}-2$ & 0.681 & 0.102 & 14.9 & 0.654 & 0.106 & 16.2 \\
\hline 298 G/T (psi) & 149.91 & 4. 20 & 2.8 & 152.76 & 2.56 & 1.7 \\
\hline
\end{tabular}

Coefficient

Standard of Variation

Mean Deviation (\&)

0 psig $(N=5)$

$\begin{array}{lll}8.16 & 19.28 & 21.9\end{array}$

\section{Total (all pressures, $\mathrm{N}=25$ )}




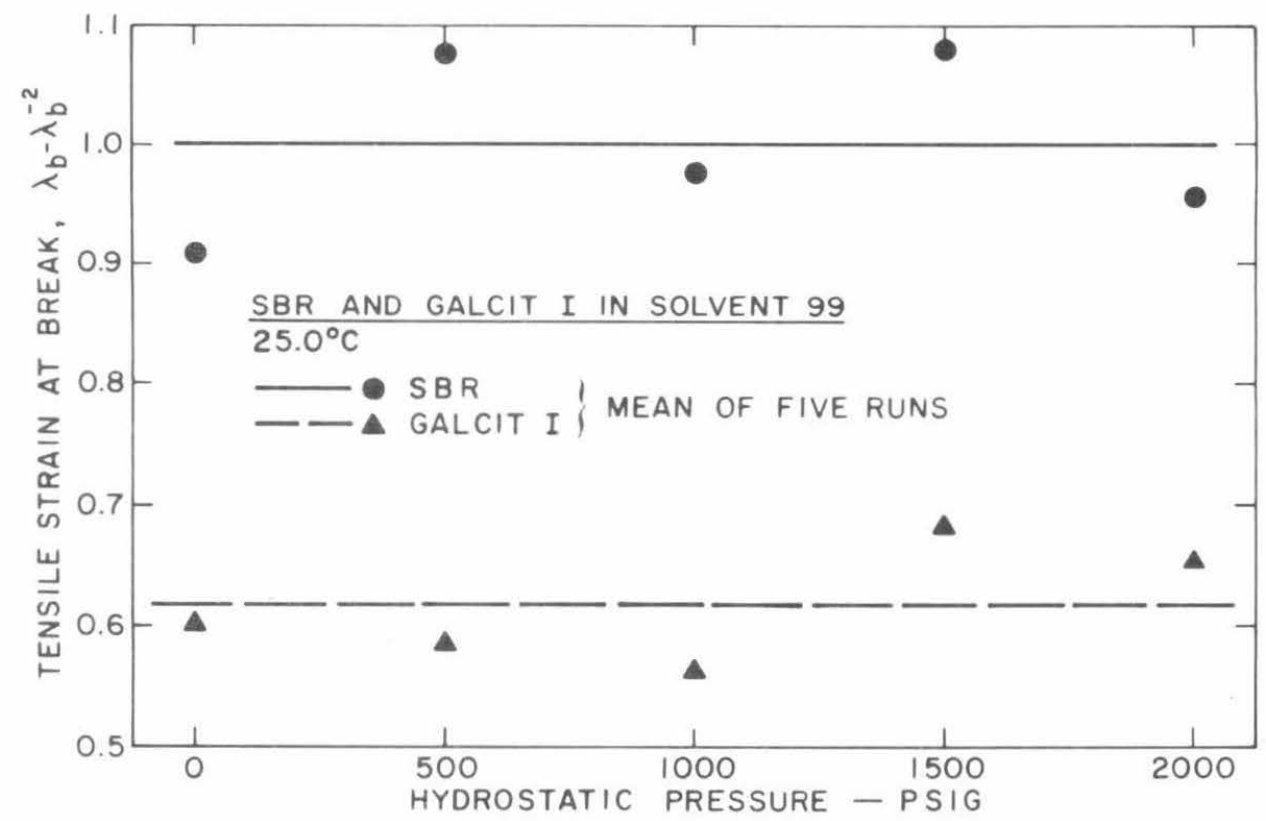

Fig. 23. Effect of Hydrostatic Pressure on the (Neohookean) Tensile Strain at Break for Swollen SBR and GALCIT I.

Tables I and II. The mean (neohookean) break strain for the swollen SBR was 1.0 , with a standard deviation of 0.19 , and a coefficient of variation of $19 \%$. The values for swollen GALCIT I were $0.62,0.12$, and $19 \%$ respectively.

The reduced tensile stress at break, $298 \sigma_{b} / T$, is plotted against the hydrostatic pressure in Fig. 24 for the swollen SBR and in Fig. 25 for swollen GALCIT I. The coefficients for the correlation between stress at break and pressure were 0.18 for the SBR and 0.36 for GALCIT I. Neither correlation is significant at the $5 \%$ level of confidence and it must be concluded that no effect of the hydrostatic pressure on the tensile break strength of the swollen rubbers could be detected within the experimental error. The mean tensile stress at break at $25^{\circ} \mathrm{C}$ of the swollen SBR was 24.3 psi with a standard deviation of 4.9 , and a coefficient of variation of 


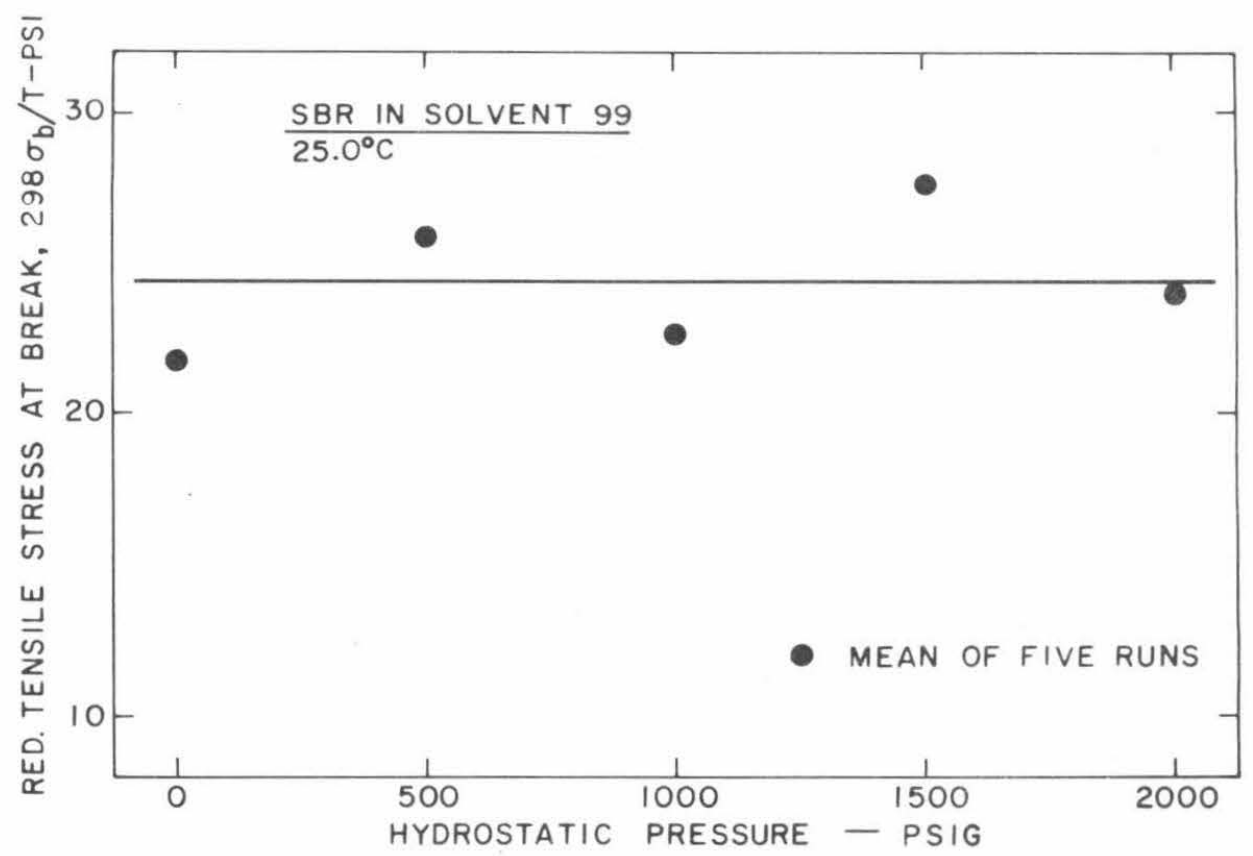

Fig. 24. Effect of Hydrostatic Pressure on the Tensile Stress at Break for Swollen SBR.

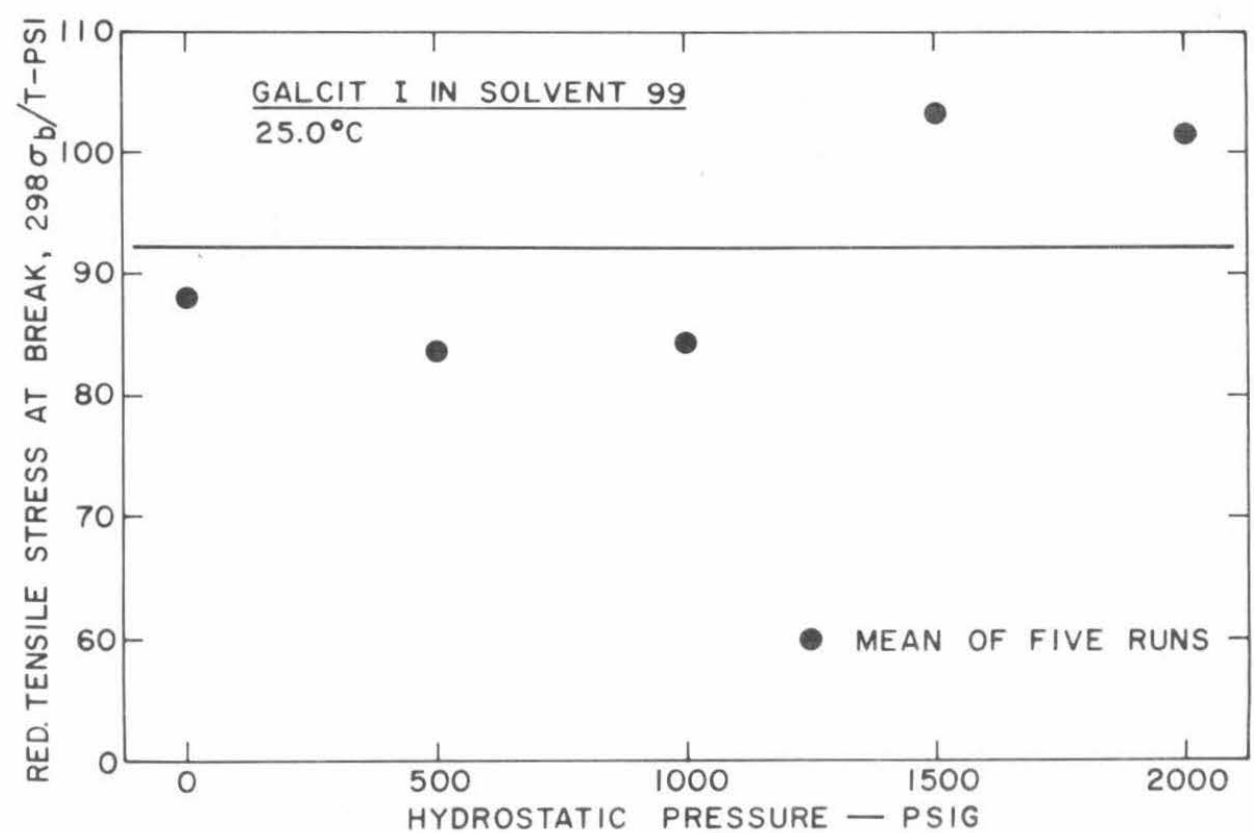

Fig. 25. Effect of Hydrostatic Pressure on the Tensile Stress at Break for Swollen GA LCIT I. 
$20 \%$. For GALCIT I the values were $92.2 \mathrm{psi}, 18.8 \mathrm{psi}$, and $20 \%$ respectively. Contrary to the modulus, there was no difference in the scatter of the break data for the two materials. Also, the strain at break showed the same scatter as the stress.

Figures 26 and 27 show plots of the reduced tensile stress at break, $298 \sigma_{\mathrm{b}} / \mathrm{T}$, against the (neohookean) tensile strain at break for the SBR and for GALCIT I, respectively. There is a total of 25 data points in each figure since five tests were run at atmospheric pressure, and at each of the hydrostatic pressures of 500, 1000, 1500, and 2000 psig. The points scatter around straight lines which simultaneously represent the failure envelopes and the equilibrium stress-strain curves for the swollen elastomers. The lines shown in Figs. 26 and 27 are lines of best fit. Their slopes represent the modulus, $G_{b}$, calculated from the break data on the assumption that the behavior is neohookean up to break at all pressures and that the hydrostatic pressure has no effect on the failure behavior. The two modulus values of $24.3 \mathrm{psi}$ for the SBR and $149.8 \mathrm{psi}$ for GALCIT I are in excellent agreement with the mean values of 24.0 and 146.6 psi obtained by averaging the individual moduli calculated from the 25 stress-strain curves.

\section{6 Failure Surfaces for the Swollen Elastomers}

It was shown in the preceding section that no effect of hydrostatic pressure up to 2000 psig could be detected within the experimental error on either the tensile stress or strain at break. This indicates that the failure surfaces for the swollen elastomers should be cylinders coaxial with the hydrostatic axis, corresponding to the Huber-Hencky-von Mises criterion, Eq. (1). 


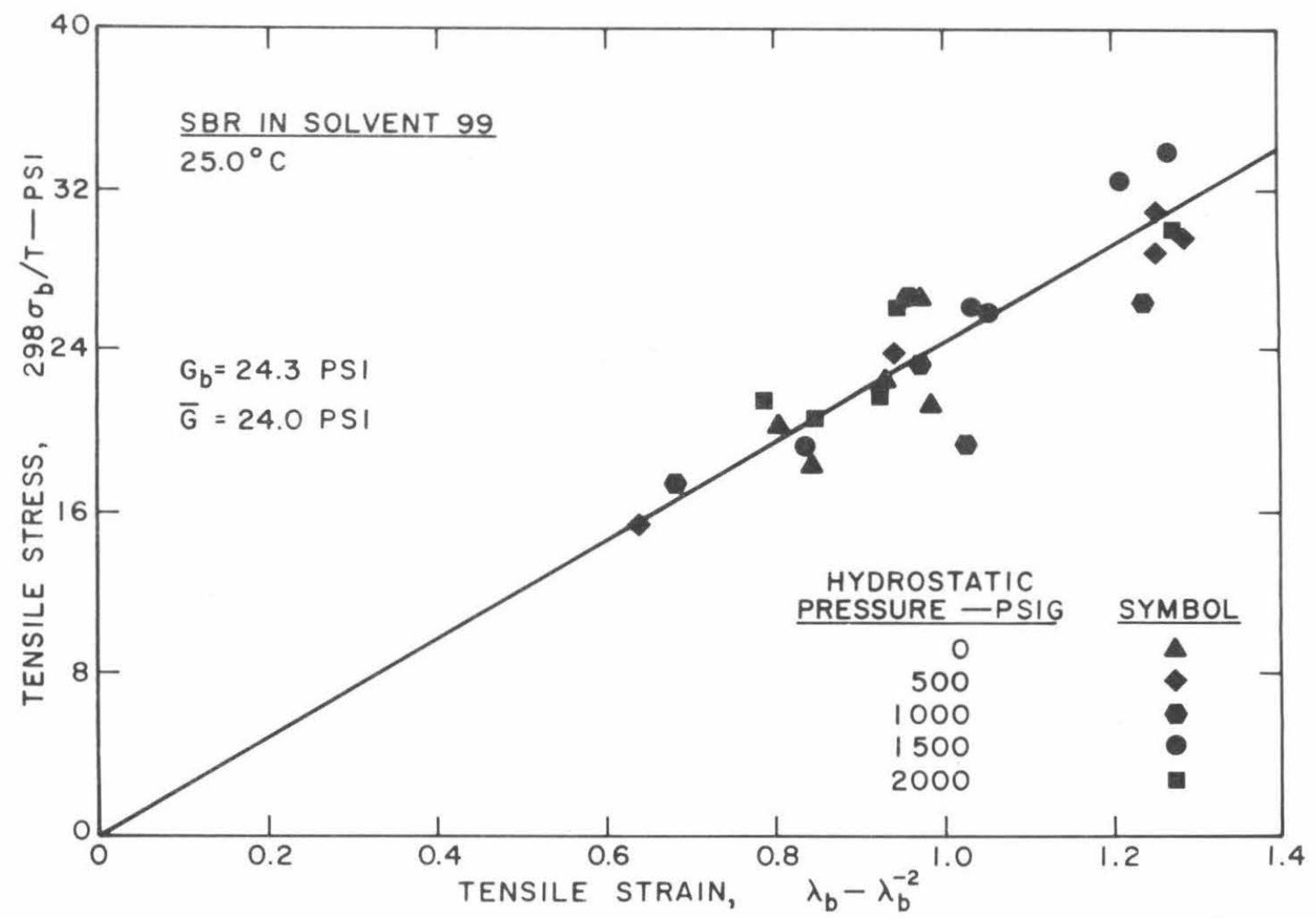

Fig. 26. Effect of Hydrostatic Pressure on Equilibrium Stress-Strain Curve for Swollen SBR at $25^{\circ} \mathrm{C}$.

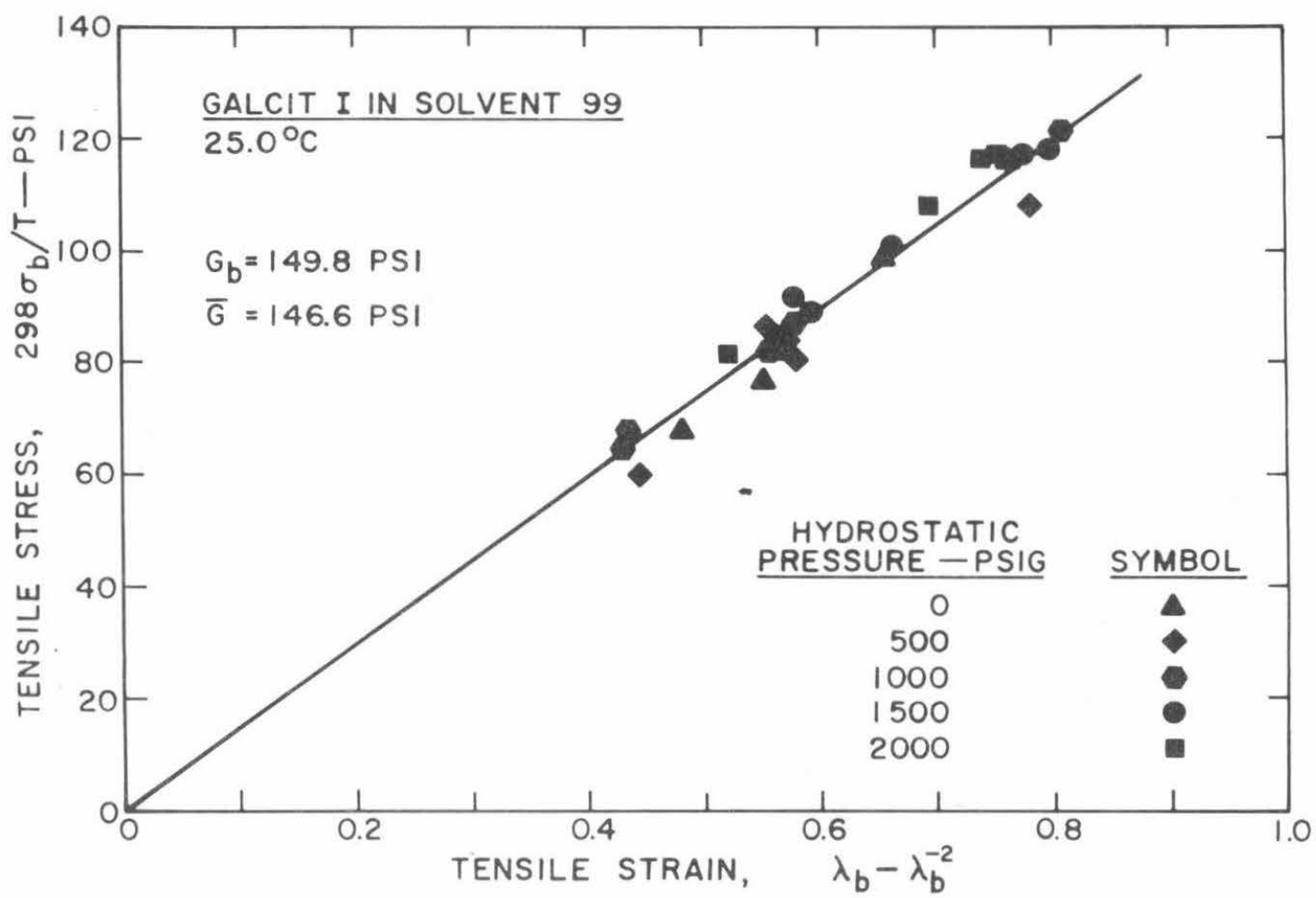

Fig. 27. Effect of Hydrostatic Pressure on Equilibrium Stress-Strain Curve for Swollen GALCIT I at $25^{\circ} \mathrm{C}$. 
As explained in more detail earlier (cf. Fig. 9 of the previous Annual Report ${ }^{(1)}$ ) a plot of the failure points in the $\sigma_{1}=\sigma_{z}$ plane reveals much about the nature of the failure surface. The coordinates of the plane are

$$
\begin{aligned}
& \sigma_{3}=-\mathrm{p}+\sigma_{\mathrm{Tb}} \\
& \sigma^{\prime}=-\sqrt{2} \mathrm{p}=\sqrt{2} \sigma_{1}=\sqrt{2} \sigma_{2}
\end{aligned}
$$

where $\mathrm{p}$ is the hydrostatic pressure, and $\sigma_{\mathrm{Tb}}$ is the tensile break stress. The plane contains both the space diagonal $\sigma_{1}=\sigma_{2}=\sigma_{3}$, and the $\sigma_{3}$-axis. Figure 28 shows the plot for the swollen SBR. Each point represents

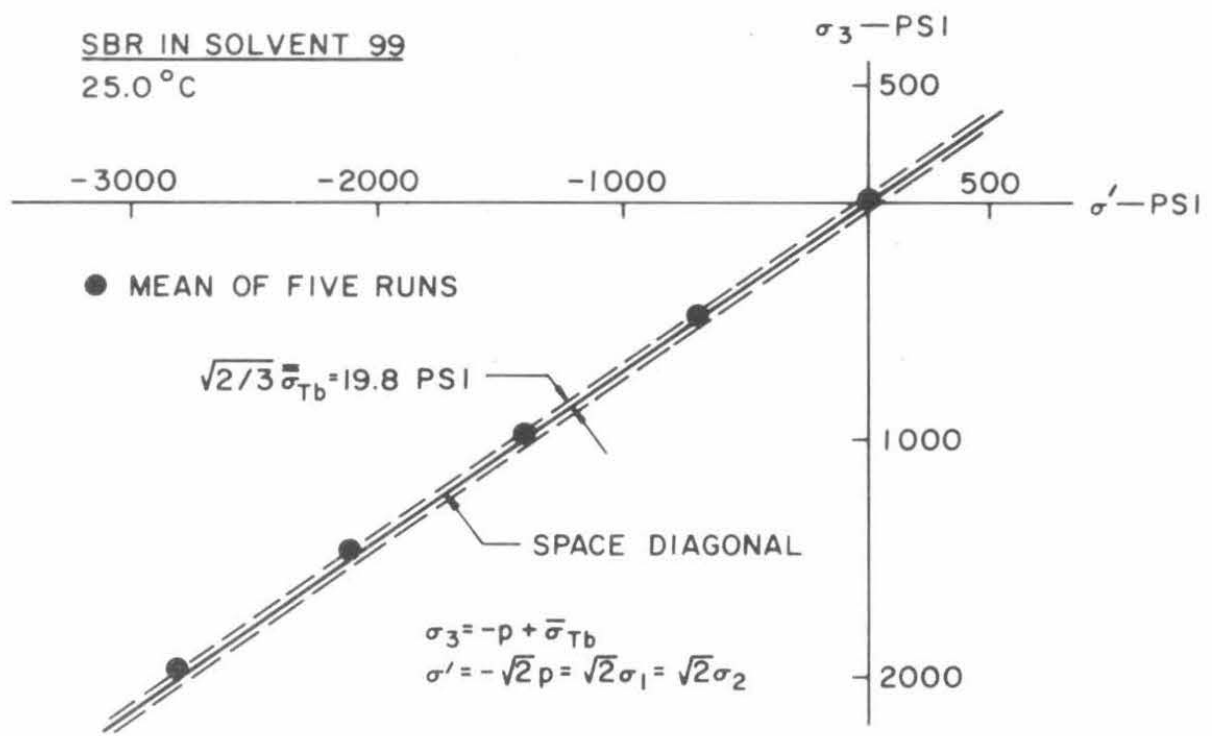

Fig. 28. Failure Stress of Swollen SBR in $\sigma_{1}=\sigma_{2}$ Plane.

the average of the five determinations made at each pressure. Within the experimental error the break points are best represented by a line parallel 
with the space diagonal. The line may be regarded as the intersection of the $\sigma_{1}=\sigma_{2}$ plane with a cylinder of radius $\sqrt{2 / 3} \bar{\sigma}_{\mathrm{Tb}}$ coaxial with the space diagonal, where $\overline{\bar{\sigma}}_{\mathrm{Tb}}$ is the grand mean of all break points.

Because the applied hydrostatic pressures were much larger than the tensile break stress of the swollen sample, the plot shown in Fig. 28 is not a sensitive one, and the corresponding data for GALCIT I are therefore not displayed in this way. A much more critical representation is obtained by plotting the deviatoric part of the (total) break stress against the isotropic (mean normal) part. As shown by Schwarzl and Staverman (18), the deviatoric and isotropic parts are given by $\sqrt{3} \sigma_{\text {os, }}$, and $\sqrt{3} \sigma_{\text {on, b' }}$, respectively. The isotropic part of any state of stress is obtained by projecting the state of stress, $\vec{\sigma}=\sigma_{1} i+\sigma_{z} j+\sigma_{3} k$, on the space diagonal. We have

$$
\sqrt{3} \sigma_{\text {on }}=\vec{\sigma} \cdot \vec{a}=\frac{1}{\sqrt{3}}\left[\sigma_{1}+\sigma_{2}+\sigma_{3}\right]
$$

where $\vec{a}$ is a unit vector along the space diagonal. The deviatoric part is obtained by projecting the state of stress onto the plane which contains the origin and is perpendicular to the space diagonal. The deviator is thus given by

$$
\sqrt{3} \sigma_{\text {os }}=\left[\left(\sigma_{1}-\sigma_{\text {on }}\right)^{2}+\left(\sigma_{2}-\sigma_{\text {on }}\right)^{2}+\left(\sigma_{3}-\sigma_{\text {on }}\right)^{2}\right]^{\frac{1}{2}}
$$

The geometric interpretation of $\sqrt{3} \sigma_{\text {os }}$ and $\sqrt{3} \sigma_{\text {on }}$ is shown in Fig. 29. 


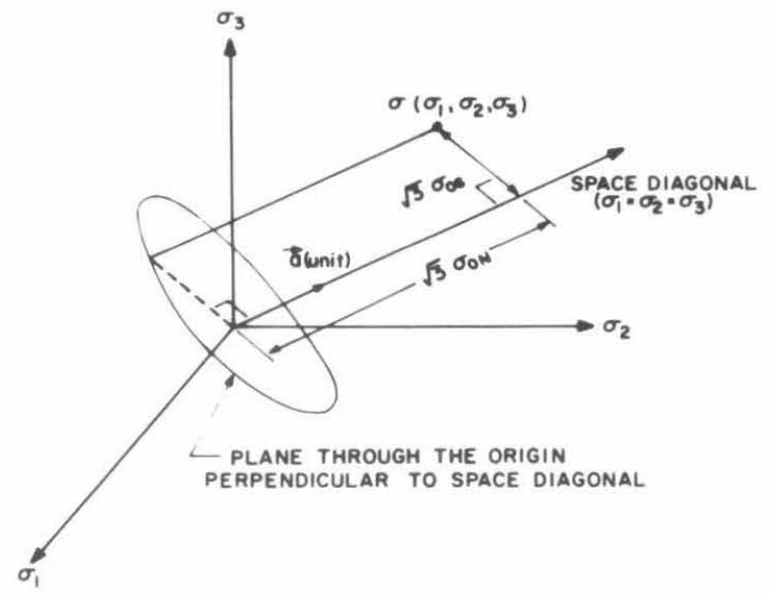

Fig. 29. Geometric Representation of Isotropic and Deviatoric Stress in Principal Stress Space.

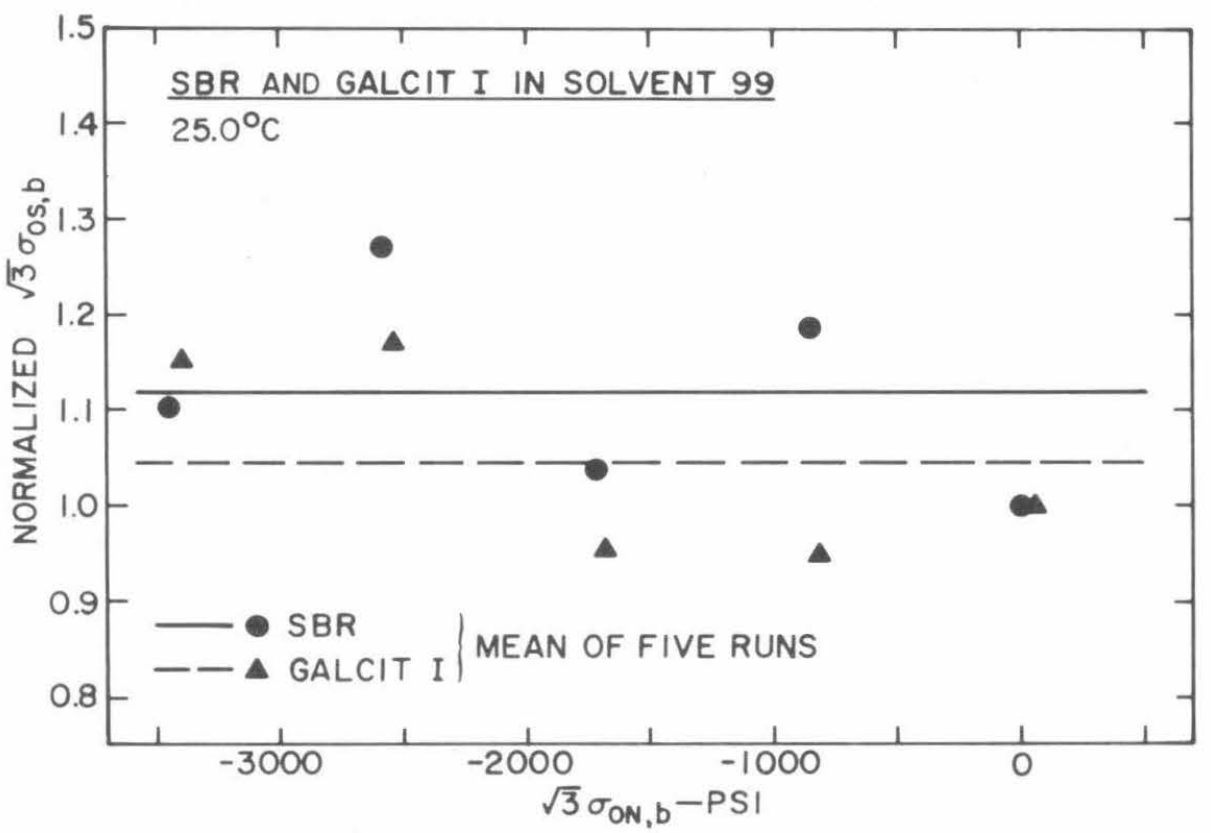

Fig. 30. Plot of Deviatoric against Isotropic Failure Stress for Swollen SBR and GA LCIT I. 
The corresponding plot for the swollen SBR and GALCIT I are shown in Fig. 30 where the octahedral shear stress at break, $\sigma_{\text {os, }}$, has been normalized to allow convenient comparison. As is clear from Fig. 29, in this plot a cylinder coaxial with the space diagonal is represented by a horizontal straight line. Within the experimental error the data for both materials indeed appear to be best represented by this criterion. Since it is unlikely that the material would withstand infinite hydrostatic tension, the cylindrical failure surface is, however, not a satisfactory representation in the first octant of principal stress space. The shape of the surface in this octant must be explored by other tests as discussed in Section 2. Measurements of greater precision over wider ranges of hydrostatic pressure may have revealed a slope or curvature more in agreement with a conical or paraboloid surface. In fact, the value of $\mathrm{m}=\sigma_{\mathrm{Cb}} / \sigma_{\mathrm{Tb}} \approx$ 3. 0 which has been reported for swollen SBR samples in toluene in the previous report ${ }^{(1)}$ favors either the cone or paraboloid surfaces over a cylindrical surface of failure. This discrepancy remains unresolved at present. 


\section{REFERENCES}

1. R. F. Landel, and N. W. Tschoegl, "A Research Program on Solid Propellant Physical Behavior," MATSCIT PS 67-1, California Institute of Technology, Pasadena, 1967. AFRPL-TR-67-193, Volume I, June 1967, pp. II-1 to II-49.

2. A. Nadai, Theory of Flow and Fracture of Solids, McGraw-Hill, New York, Vol. I, 1950, 207.

3. J. C. Jaeger, Elasticity, Fracture and Flow, Methuen, London, 1956.

4. J. Marin, Mechanical Behavior of Engineering Materials, PrenticeHall, Englewood Cliffs, New Jersey, 1962, 112.

5. S. Timoshenko, Strength of Materials, D. Van Nostrand, New York, Part II, 1956, 444.

6. R. L. Thorkildsen, and W. V. Olszewski, "The Effect of Biaxial Stresses on the Deformation and Fracture of Polymethylmethacrylate," Advanced Tech. Lab. Rep. 61GL181, General Electric Co., Schenectady, April 1962.

7. R. E. Ely, "Biaxial Stress-Strain Behavior of Nylon Tube Specimens," U. S. Army Missile Command, Redstone Arsenal, Alabama, Rep. RR-TR-64-11, 1964.

8. R. E. Ely, Polymer Eng. and Sci., 7, 40 (1967).

9. F. Stassi-D'Alia, "Limiting Conditions on Yielding of Thick Walled Cylinders and Spherical Shells, "Universita di Palermo, Italy, Contract DA-91-591-EUC-1315, OI-4263-60, 1959.

10. T. L. Smith and J. A. Rinde, "Ultimate Tensile Properties of Elastomers. V.Rupture in Constrained Biaxial Tension," Submitted to J. Pol. Sci., Part A-2.

11. C. K. Lim, "Failure Criteria for Viscoelastic Materials under Biaxial Stress Fields," Ph. D. Thesis, The Pennsylvania State University, University Park, Pennsylvania, 1967.

12a. W. L. Ko, "Application of Finite Elastic Theory to the Behavior of Rubberlike Materials," Ph. D. Dissertation, California Institute of Technology, 1963.

12b. R. F. Landel, and R. F. Fedors in: B. Rosen, Fracture Processes in Polymeric Solids, IIIB, Interscience, 1964, Fig. 57 (after Ko).

13. P. J. Blatz, and W. L. Ko, Trans. Soc. of Rheology, 6, 223 (1962).

14. P. J. Blatz, Rubber Chem. Tech., 36, 1459 (1963). 
15. M. G. Sharma, and C. K. Lim, Polymer Eng. and Sci., 5, 254 (1965).

16. T. M. Jones, and R. B. Kruse, J. Spacecraft and Rockets, $\underline{3}, 265$ (1966).

17. J. W. Jones, and W. G. Knauss, "Propellant Failure Criteria," AIAA preprint 65-157, presented at the AIAA 6th Solid Propellant Rocket Conference, Washington, D. C., Feb. 1965.

18. F. R. Schwarzl, and A. J. Staverman, in: H. A. Stuart, Die Physik der Hochpolymeren, Vol. IV, Chapter 3, Springer, Berlin, 1956. 

PART II

ON THE PACKING OF SPHERES OF DIFFERENT

DIAMETERS IN SPACE

H. Kausch

and

N. W. Tschoeg1 


\section{PART II \\ ON THE PACKING OF SPHERES OF DIFFERENT \\ DIAMETERS IN SPACE}

\section{CONTENTS}

1. Introduction. . . . . . . . . . . . . . . . II - I

2. Regular Packing of Spheres in Space . . . . . . . II - 2

3. Close Packing of Circles in a Plane . . . . . . . II - 6

3. 1 Packing Geometry of Regular and Irregular Interstices . . . . . . . . . . . . . . II - 7

3. 2 Distribution of Radii in Two-dimensional Closepacking. . . . . . . . . . . . . II -10

3. 3 Analytical Function for $\mathrm{n}(\mathrm{r})$. . . . . . . . . II - 13 3. 4 Random Packing . . . . . . . . . . . . II - 19

4. Two- and Three-Dimensional Particle Size Distributions II - 20 References . . . . . . . . . . . . . . . . III 29 
ILLUSTRATIONS

Fig. 1. Close Packing of Circles of Uniform Diameter • . II - 7

Fig. 2. Close Packing of Circles of Three Different

Diameters. • . . . . . . . . . . . . II - 7

Fig. 3. Regular Interstice . . . . . . . . . . . . II - 8

Fig. 4. Irregular Interstice . . . . . . . . . . . . II - 9

Fig. 5. Cumulative Distribution of 7th Order Circles . . . II - 10

Fig. 6. Geometric Mean of Circle Radii of One Generation as a Function of the Order of Generation . . . . II - 12

Fig. 7. Normalized Distribution of Circle Radii

Regular and Irregular Interstice . . . . . . . II - 13

Fig. 8. Distribution of 7 th Order Circle Radii . . . . . II -17

Fig. 9. Distribution of Circle Radii for Three Different

Step-distributions of Sphere Radii. . . . . . . II - 22

Fig. 10. Distribution of Sphere Radii Calculated from

Optimal Circle Radii Distribution . . . . . . . II - 28 


\section{TAB LES}

Table I Packing Densities of Hard Inactive Spheres in Space . . . . . . . . . . . . . II -4

Table II Calculated Distribution of 288 Largest Spheres for Maximal Filling of Hexagonal Unit Cell. . . . II - 5

Table III Degree of Filling in Two-dimensional Closepacking. . . . . . . . . . . . . . . II -11

Table IV Cumulative Distribution of Circle Radii . . . . . II - 24 


\section{Introduction}

A major problem in the technology of filled elastomers (solid propellant grains) is the incorporation of a sufficiently large volume fraction of solids into an elastomeric binder so as to obtain a maximum energy output per weight of propellant. Elastomeric binder and solids fraction are chosen accordingly. Pertinent properties of the solids fraction are the chemical nature, shape distribution and the size distribution of the solid particles. The ballistic requirements of a solid propellant grain usually determine the nature of the solids fraction (metal, oxidizer). The particle shape distribution is in many cases prescribed by the grinding or milling process employed to produce the filler. So the particle size distribution remains virtually the only disposable parameter. The influence of bimodal or multimodal distributions on regular packing of particles can be visualized readily. It is well-known that not only the packing fraction ${ }^{(1-6)}$ but also the viscosity of highly concentrated solutions ${ }^{(3-6)}$ - such as the slurries of uncured filled rubbers - and the ultimate properties (stress and strain at break) of the grain ${ }^{(6)}$ depend upon the size distribution of the solids fraction. Interest in the statistics and geometry of packing of particles is not, however, confined to one branch of science. Metallurgists, petrographers, chemical engineers, statisticians, and even traffic experts have examined packing problems from different viewpoints. The literature on this subject is very extensive and, because it is widely scattered, is not easily accessible. It appears, however, that there is no theory available which describes the random packing of a multimodal distribution of even the simplest particles, i.e., spheres. 
This report describes the results obtained thus far in an attempt to come to terms with this problem. In search for optimal sphere size distributions, an investigation was first made of the sizes of spheres which would successively fill the interstices in regular arrays of uniform spheres. This work is described in Section 2. It will be shown there, however, that the spectrum of sphere sizes which can be determined unambiguously covers only a small range. Section 3 deals with the comparable process of packing circles of ever decreasing diameter in two-dimensional interstices. This can be accomplished unambiguously and leads to a spectrum of well-defined circle radii.

Any distribution of circles in a plane can be interpreted as the twodimensional cross-section through an aggregate of spheres in space. Section 4 describes the close relations which were found to exist between the packing densities and distribution functions of randomly packed circles in a plane and spheres in space. If the two-dimensional packing is known, the three-dimensional packing can be derived. Section 4 makes use of these relations to transform the calculated optimal distribution of circle sizes into the equivalent distribution of spheres in space.

\section{Regular Packing of Spheres in Space}

The volume fraction, $\varphi$, of regular arrays of spheres can be calculated readily, and thus the increase in packing fraction of a polydisperse sphere size distribution over a monodisperse distribution can be studied.

For monodisperse spheres the maximum degree of volume filling $(\varphi=0.7405 \%)$, is realized in either hexagonal or cubid close packing. If 
the interstices of such a packing, $(1-\varphi)$, are filled in the same way with smaller spheres the obtainable volume loading as a function of the number, $k$, of sphere sizes should be

$$
\varphi_{\mathrm{k}}=1-(1-\varphi)^{\mathrm{k}}
$$

Since each smaller sphere size should practically behave like a liquid in filling the interstices in the lattice of the next larger sphere size large size differences are required between successive components. Furnas (1) developed a semi-theoretical equation for $\varphi_{\mathrm{k}}$ for random packings on the basis of this idea.

Packing densities calculated or experimentally observed by different workers for hard, inactive spheres are assembled in Table $I$ where $k$ is the number of the generation of spheres and $r_{1}: r_{k}$ is the ratio of the radius of the largest to that of the kth sphere size. Table I shows that the calculated packing fractions increase with the number of different sizes of the packing spheres. It also shows that the packing fractions are higher, if the differences between the largest and smallest spheres are large. The experimentally observed densities of random packings are about 10 to 15 percent smaller than the densities for regular packing of the same sphere size distribution.

Even though rather high volume fractions can be achieved with a small number of widely differing sphere sizes, it has been shown ${ }^{(6)}$ that such a simple system leads to values of low stress and strain at break. A closer distribution of sphere radii is possible if the interstices of a regularly packed array are filled with spheres of the largest possible radius. In an array of hexagonally close packed spheres of radius 1.0 
TABLE I

Packing Densities of Hard Inactive Spheres in Space

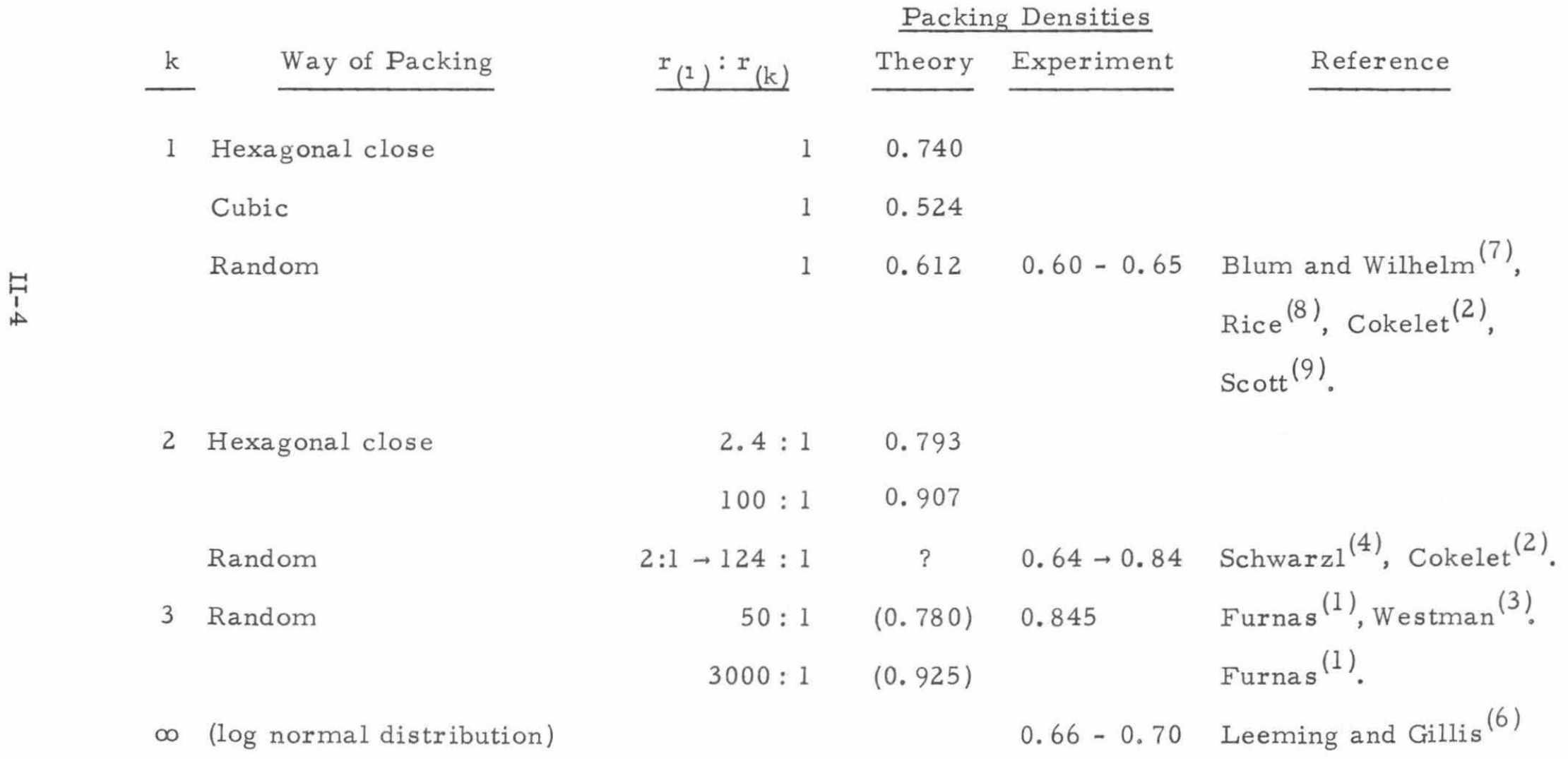


there will be "square" and "triangular" interstices which can accommodate second generation spheres of radius 0.414 and 0.225 respectively. After the second generation has been placed, however, the spaces to be filled will always be "rectangular" and so the placement of further spheres is ambiguous.

Table II shows the distribution of the radii of the 288 largest spheres within a unit cell.

TABLE II

Calculated Distribution of 288 Largest Spheres for Maximal Filling of Hexagonal Unit Cell

\begin{tabular}{|c|c|c|c|c|c|}
\hline $\mathrm{k}$ & $\mathrm{R}$ & $N(R)$ & $\Delta \varphi(\%)$ & $\begin{array}{c}\varphi(\%) \\
\text { (this work) }\end{array}$ & $\begin{array}{c}\varphi(\%) \\
\text { (Ref. } 10) \\
\end{array}$ \\
\hline 1 & 1.000 & 4 & 74.05 & 74.05 & 74.05 \\
\hline 2 & 0.414 & 4 & 5.26 & 79.31 & 79.3 \\
\hline 2 & 0.225 & 8 & 1.68 & 80.99 & 81.0 \\
\hline 3 & 0.177 & 32 & 3.26 & 84.25 & 84.2 \\
\hline 4 & 0.124 & 32 & 1.12 & 85.38 & 85.1 \\
\hline 4 & 0.111 & 16 & 0.40 & 85.77 & -- \\
\hline 5 & 0.081 & 48 & 0.47 & 86.25 & -- \\
\hline 5 & 0.080 & 48 & 0.45 & 86.70 & -- \\
\hline 5 & 0.072 & 96 & 0.66 & 87.35 & -- \\
\hline
\end{tabular}

The first three columns contain the number of generation of spheres, $k$, the radius, $R$, and the frequency, $N(R)$. The individual and cumulative volume fractions, $\Delta \varphi$ and $\varphi$, are shown in the next two columns. For comparison the volume fractions obtained by Horsfield ${ }^{(10)}$ are also given. 
The ambiguity of the placement of speres shows up in the slight differences for the volume fractions of the third and fourth generation.

About $84 \%$ of the volume can be filled unambiguously. This corresponds to a range of sphere sizes of about 1:6. Beyond this, unambiguous results can not be obtained.

\section{Close Packing of Circles in a Plane}

Since their packing in a plane can be treated unambiguously, a study was made of the close packing of circles. This procedure is of practical value in view of the equivalence of line, area, and volume fractions in random packing. The conclusions reached in an investigation of the packing of circles in a plane can thus be transferred directly to the packing of spheres in space.

The equivalence of volume, area and line fraction can be demonstrated by the following considerations. If a random assembly of particles of arbitrary size and shape distribution fills a fraction $\varphi_{\mathrm{V}}$ of any subvolume which is sufficiently large compared with the particle size, then any plane through this subvolume will contain an area fraction $\varphi_{\mathrm{A}}$ which is occupied by bisected particles. The area fraction $\varphi_{\mathrm{A}}$ is equal to the volume fraction $\varphi_{\mathrm{V}}$. It is easily realized that a stack of planes, each having area fraction $\varphi_{\mathrm{A}}$, when piled upon each other, will give a volume fraction $\varphi_{\mathrm{V}}=\varphi_{\mathrm{A}}$. Similarly, if a cross-section through a random assembly is divided into straight lines, the fraction of lines passing through the particles, $\varphi_{\mathrm{L}}$, is equal to $\varphi_{\mathrm{V}}$ and $\varphi_{\mathrm{A}}$.

The equality of all three fractions was first proposed by Rosiwal

and experimentally verified by several other authors $(12,13)$. However, 
no simplification results from a consideration of the packing of linear elements in a line instead of the area or volume packing since any distribution of linear elements can be arranged on a continuous line.

\section{3, 1 Packing Geometry of Regular and Irregular Interstices}

Our first problem will be to find the optimal distribution, i. e., the distribution of circle sizes which can be packed to cover the largest possible area with the fewest possible number of circles. The circles are considered to be disks of vanishing thickness and overlapping is not permitted. The regular packing of circles of one diameter is demonstrated in Fig. 1. Close packing of circles of three different diameters is shown in Fig. 2. This packing is achieved when the radii are in the ratio of $r_{1}: r_{2}: r_{3}=1: 0.464: 0.268$. The interstices between the circles are to

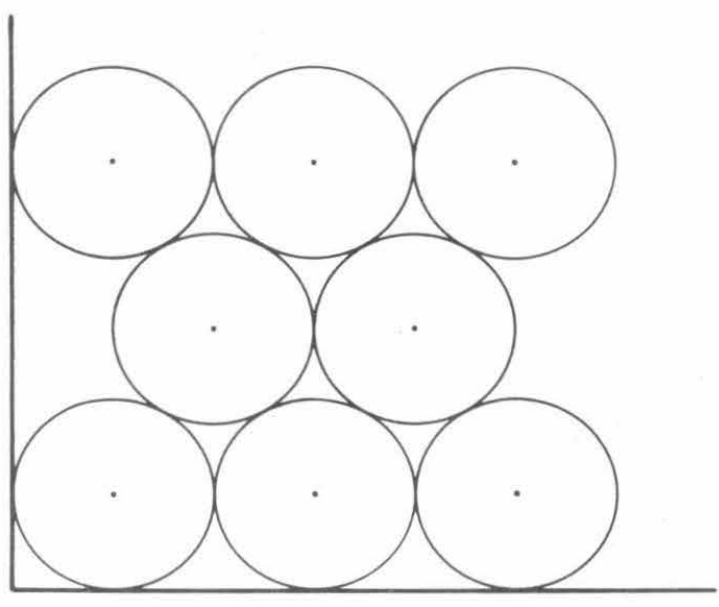

Fig. 1. Close Packing of Circles of Uniform Diameter.

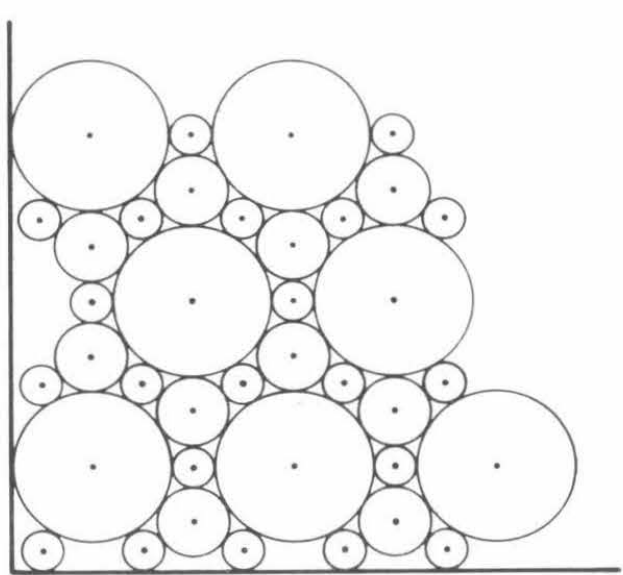

Fig. 2. Close Packing of Circles of Three Different Diameters.

be filled with circles of smaller diameter. If this is done, new but smaller interstices are created which in turn may be filled by another generation of 
of circles. In this way a discrete distribution of circle radii is established. If the lower limit of this distribution is extended to zero, the packing fraction approaches unity.

The choice of the upper limit of sphere sizes is free and does not affect the problem investigated here. In practice there will be a maximum particle size which will depend on the particular application. The lower limit of zero particle size cannot, of course, be realized physically. However, once the contribution of higher orders of circles to the total packing fraction has been investigated, a lower practical limit can be chosen. Selection of an upper and lower limit leads to a truncated distribution characterized by a definite area fraction.

The shape and number of the interstices depend upon the choice of the first generation $(k=1)$ of circles. If these are uniform, the regular packing leads to regular interstices as shown in Fig. 3.

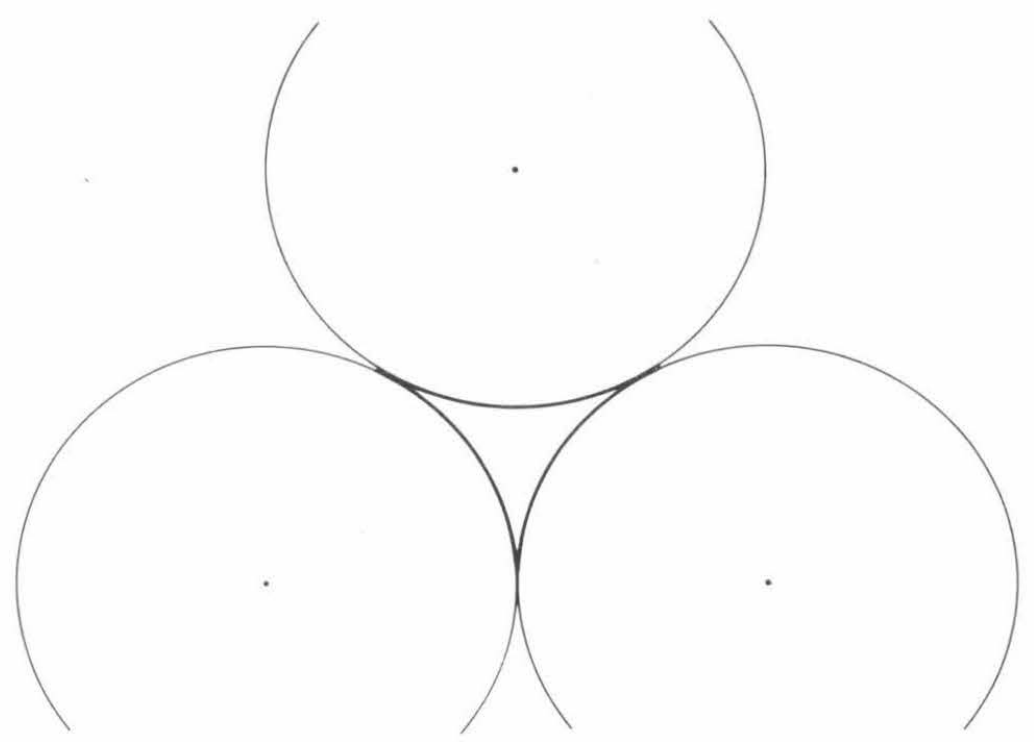

Fig. 3. Regular Interstice. 
If the first generation of circles consists of different diameters, irregular interstices of the type shown in Fig. 4 are formed. The highest

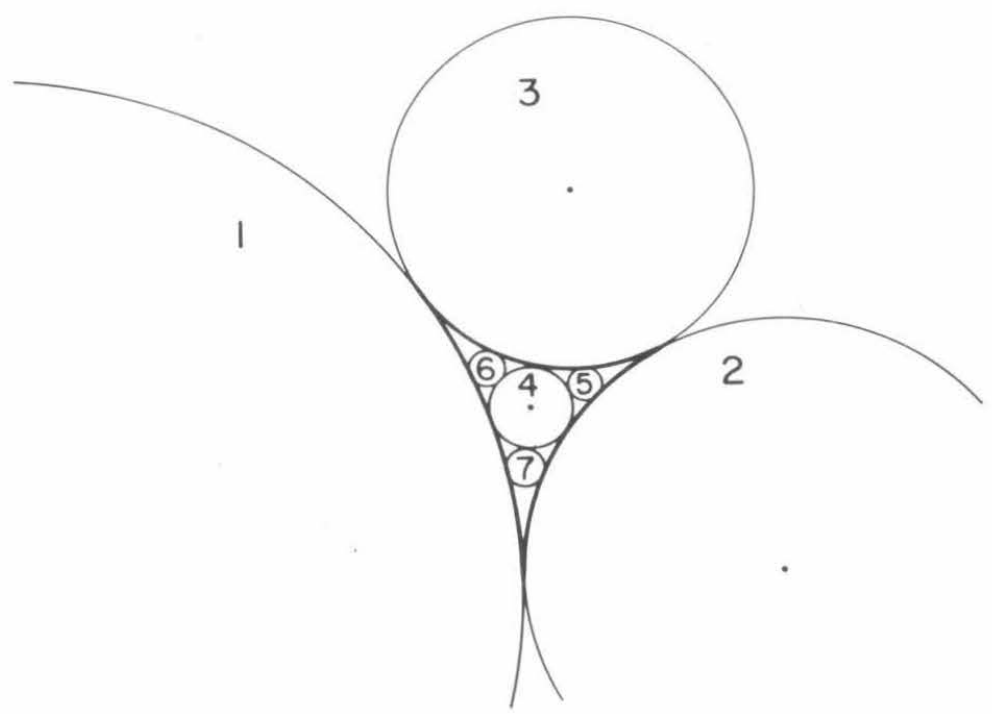

Fig. 4. Irregular Interstice.

degree of filling of any interstice by a single circle will result when it touches each of the first generation circles*. If the radii of the three first order circles are denoted by $r_{1}, r_{2}, r_{3}$, and that of the touching circle by $r_{4}$, then

$$
r_{4}=\frac{r_{1} r_{2} r_{3}}{r_{1} r_{2}+r_{2} r_{3}+r_{1} r_{3}+2 \sqrt{r_{1} r_{2} r_{3}\left(r_{1}+r_{2}+r_{3}\right)}}
$$

Placing the second generation circle of radius $r_{4}$ into the interstice creates three smaller interstices which are bounded by $r_{1}, r_{2}, r_{4} ; r_{1}, r_{3}, r_{4}$; and $r_{2}, r_{3}, r_{4}$ respectively. The largest circles which will fit into the se smaller interstices will have radii $r_{5}, r_{8}$, and $r_{7}$. The values of the radii * A simple maximum-minimum calculation shows that a maximum fraction of an interstice is covered by two circles if one touches all three confining circles, and the other touches the larger two of these (cf. also Ref. 14). 
of this third generation of circles can be obtained from Eq. (2) by the proper substitutions. The number of circles within a generation increases by a factor of three with each step. A simple computer program allows the calculation of the radii for as many generations as desired.

\section{2 Distribution of Radii in Two-dimensional Closepacking}

The distribution of radii of smaller circles filling all interstices optimally was determined up to the seventh order for the regular and irregular interstices shown in Figs. 3 and 4.

The distributions of the seventh order circles for these two arrangements are plotted semi-logarithmically in Fig. 5. These are

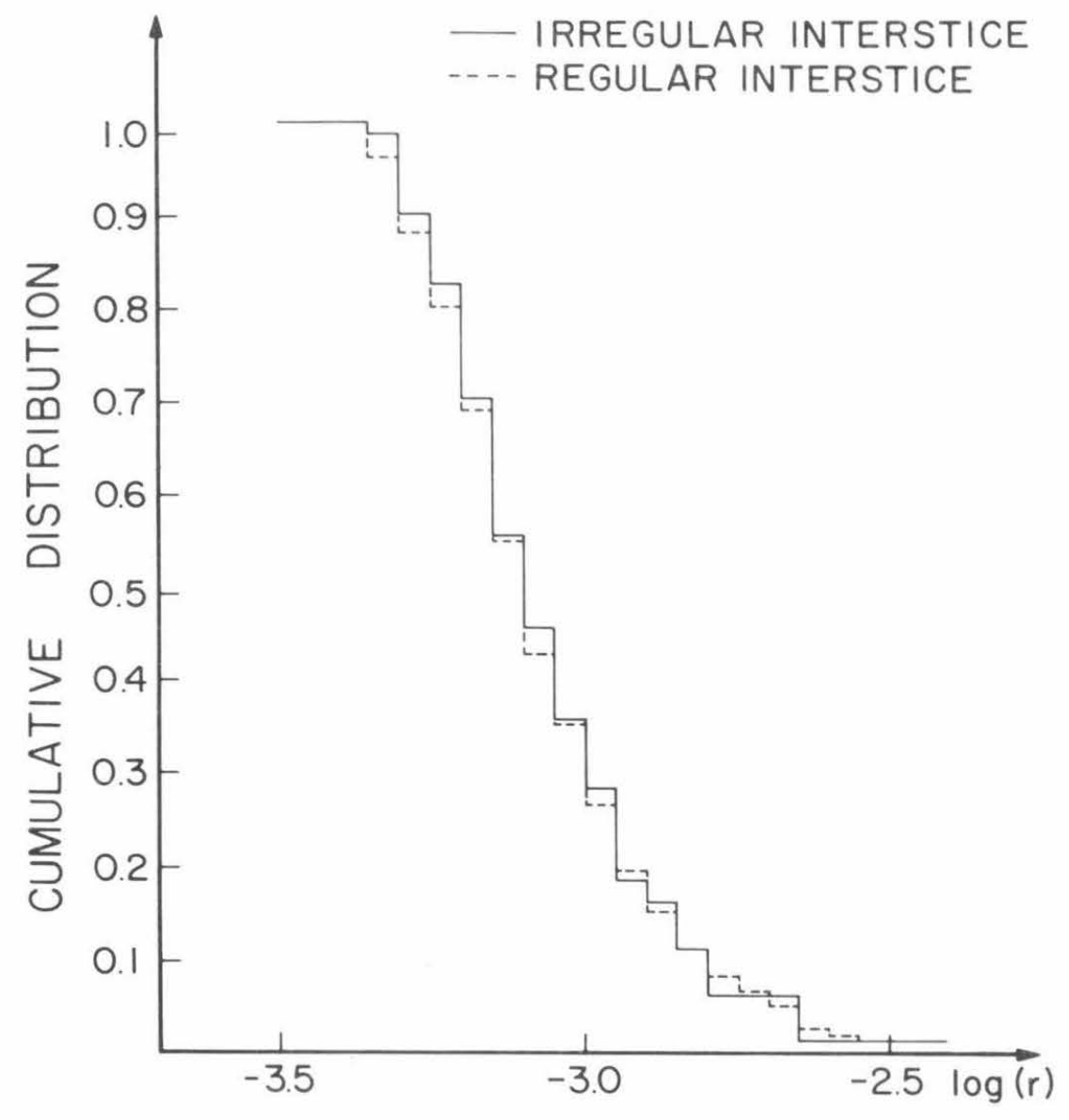

Fig. 5. Cumulative Distribution of 7 th Order Circles. II- 10 
typical of the distributions of any higher order of circles. It is seen that there is hardly any difference in distribution whether we start from a regular or an irregular interstice.

In Table III the degree of filling, (or area fraction), of the interstice obtained from each generation of $\operatorname{circles,} \Delta \varphi$, and the cumulative degree of filling, $\varphi$, are listed. Again it may be seen that the values for the regular and the irregular interstice are sensibly identical. This is

TABLE III

Degree of Filling in Two-dimensional Closepacking

\begin{tabular}{|c|c|c|c|c|c|}
\hline \multirow[b]{2}{*}{$\mathrm{k}$} & \multirow{2}{*}{$\begin{array}{c}\text { Number of } \\
\text { Circles } \\
\end{array}$} & \multicolumn{2}{|c|}{ Regular Interstice } & \multicolumn{2}{|c|}{ Irregular Interstice } \\
\hline & & $\Delta \varphi(\%)$ & $\varphi(\%)$ & $\Delta \varphi(\%)$ & $\varphi(\%)$ \\
\hline 2 & 1 & 46.6 & 46.6 & 46.3 & 46.3 \\
\hline 3 & 3 & 23. 0 & 69.7 & 23. 1 & 69.4 \\
\hline 4 & 9 & 12.3 & 82.0 & 12. 4 & 81.8 \\
\hline 5 & 27 & 6. 9 & 88.9 & 7. 0 & 88.8 \\
\hline 6 & 81 & 4. 1 & 93.0 & 4. 1 & 92.9 \\
\hline 7 & 243 & 2. 4 & 95.4 & 2. 5 & 95.4 \\
\hline
\end{tabular}

also brought out in Fig. 6, where the geometric mean of the radii of each generation is plotted logarithmically against the order of generation. This plot insures that the relation between individual orders are invariant to multiplication of all radii by a constant factor. The rather unexpected result is that $\langle\ln (\mathbf{r})\rangle$ decreases with each generation by a constant amount of 0.9075 (corresponding to a factor of 0.4035 ) no matter whether the first generation of circles forms a regular or an irregular interstice. From Figs. 5 and 6 the conclusion may be drawn that the calculated distribution 


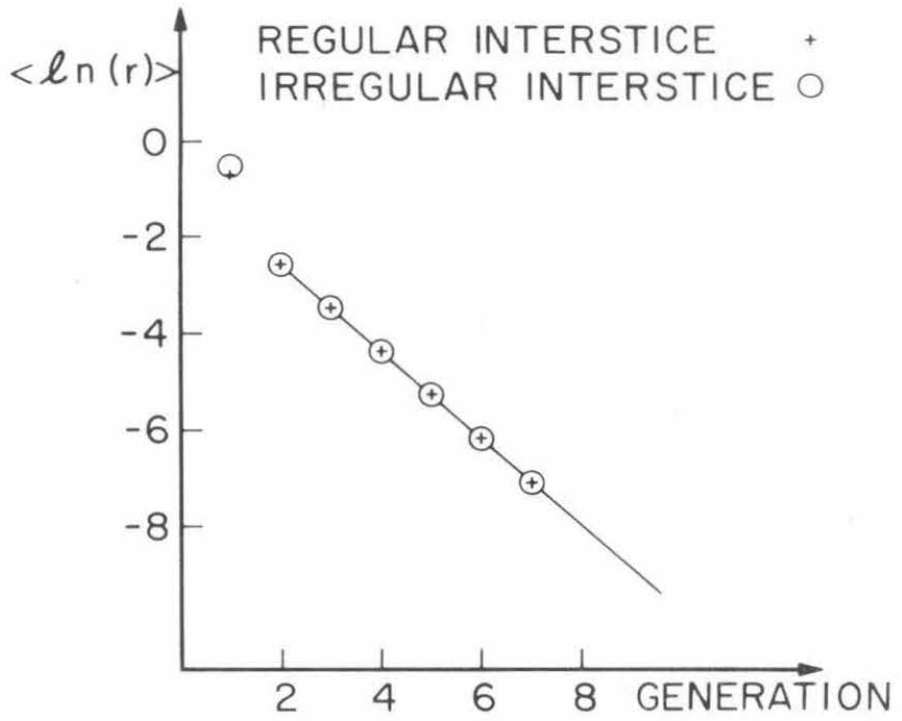

Fig. 6. Geometric Mean of Circle Radii of One Generation as a Function of the Order of Generation.

of circles which lead to a maximum degree of filling of the interstice is almost the same for any arrangement of first order circles - provided the values of $\mathrm{r}_{4}$ are identical and that all circles touch.

Once the distribution of circles which will fill the

original interstices optimally has been established a normalized distribution for the filling of any area can also be obtained. To achieve this, the number and size of the first order circles and of the interstices formed between them must be known. A regular array of circles $r_{1}$ as shown in Fig. 1 has a unit cell of $4 \sqrt{3} r_{1}{ }^{2}$ which contains two circles $r_{1}$ and four interstices. The number of circles $r_{1}$ per unit area is therefore $2 / 4 \sqrt{3} \mathrm{r}_{1}{ }^{2}=0.2887 / \mathrm{r}_{1}{ }^{2}$, and the number of interstices or circles $\mathrm{r}_{4}$ is $0.5774 / \mathrm{r}_{1}^{2}$. The number of circles of higher order increases by a factor of three per generation. The normalized intensities for an array with irregular interstices are derived in the same way to be: 


$$
\begin{aligned}
& n\left(r_{1}\right) r_{1}^{2}=0.1796 \\
& n\left(r_{2}\right) r_{1}^{2}=0.3591, \\
& n\left(r_{3}\right) r_{1}^{2}=0.5387 \\
& n\left(r_{4}\right) r_{1}^{2}=2.1547 .
\end{aligned}
$$

The normalized intensities of the first few generations of circles are shown in Fig. 7 for arrays with regular and irregular interstices.

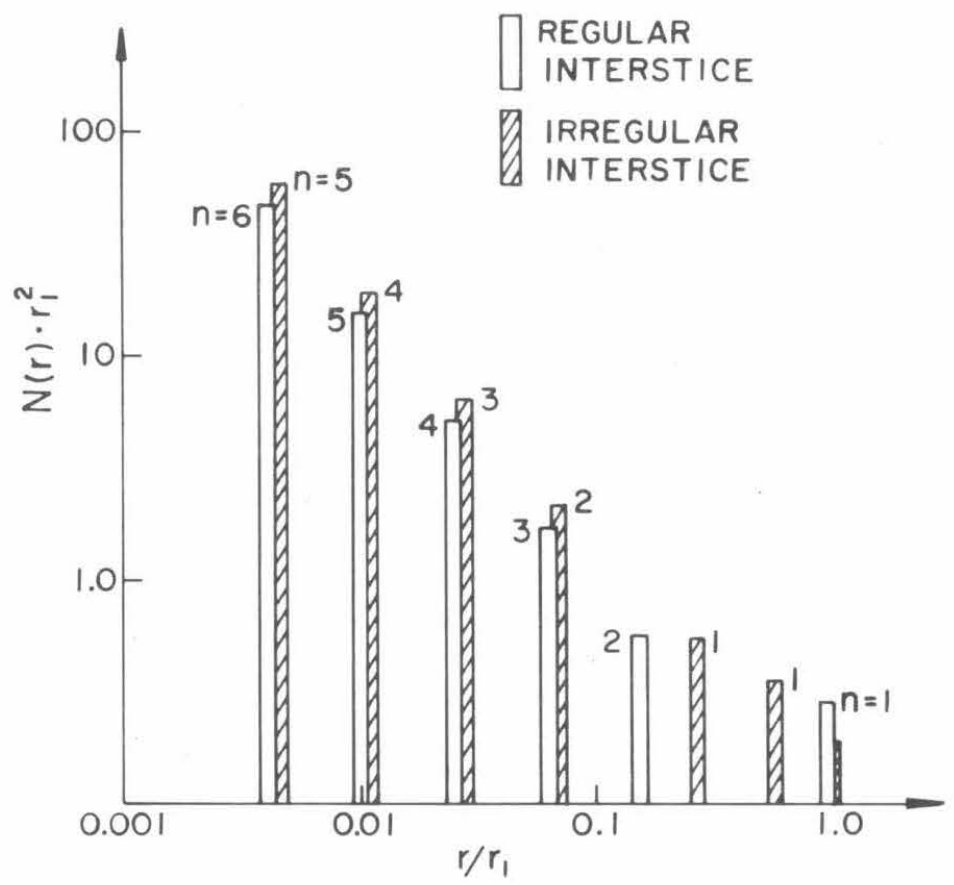

Fig. 7. Normalized Distribution of Circle Radii Regular and Irregular Interstice.

\section{3 Analytical Function for $n(r)$}

In view of the obviously very general character of the distribution of radii within one generation it is desirable to express it by an analytical function. To do so, we must first examine Eq. (2) in more detail. 
We have assigned $r_{1}$ to the largest and $r_{3}$ to the smallest of the three first order circles. Obviously $r_{4}$ will be determined mainly by $r_{3}$. The lower limit of the ratio $r_{4} / r_{3}$ is obtained when the first order circles are uniform. Its value is $1 /(3+2 \sqrt{3})=0.155$. The upper limit is unity, and is obtained when $r_{1}$ and $r_{2}$ are very much larger than $r_{3}$. For a wide range of values of $r_{1} / r_{3}$ and $r_{2} / r_{3}$ the ratio $r_{4} / r_{3}$ is close to 0.35 .

Except for first order circles, geometry forbids that two or more circles of the same generation touch each other. Therefore, the interstices are formed by circles of different generations for which $r_{4} / r_{3}$ assumes neither of the extreme values. This means that the three circles which can be placed around each circle of radius $r_{(k-1)}$ of the $(k-1) s t$ generation, have radii of about $0.35 \mathrm{r}_{(\mathrm{k}-1)^{\circ}}$ The smallest circle within the kth generation will be found in the interstices which are enclosed by one circle each of the $(k-1) s t,(k-2)$ nd and $(k-3)$ rd generation. If we assume that the radii of these circles decrease by a constant factor of A per generation we obtain the following conditions:

$$
\begin{aligned}
& r_{2} / r_{1}=A \\
& r_{3} / r_{1}=A^{2} \\
& r_{4} / r_{1}=A^{3}
\end{aligned}
$$

$\left(r_{1}\right.$ now stands for $r_{(k-3)}$ etc., so Fig. 4 can be used for illustration of the situation). Substitution of Eqs. (3) into Eq. (2) gives $A=0.3460$.

It is of interest to calculate the ratios $r_{5} / r_{4}, r_{6} / r_{4}$ (cf. Fig. 4) also. We obtain 


$$
\begin{aligned}
& r_{5} / r_{4}=0.3460 \\
& r_{6} / r_{4}=0.3766 \\
& r_{7} / r_{4}=0.5060
\end{aligned}
$$

We may see from Eqs. (4) that $r_{5}$, the radius of the smallest circle in the $(k+1)$ generation, is smaller than $r_{4}$ by a factor of 0.3460 . This is in accordance with the assumption of a constant decrease of the radii from generation to generation. The radius, $r_{B}$, of the next smaller circle which is surrounded by members of the generations $k, k-1$, and $k-3$, is slightly larger than $r_{5}$. Although $r_{7}$ is surrounded by the largest of the original circles, it is still only $0.5060 \mathrm{r}_{4}$. This value is changed only slightly if $r_{1}$ and $r_{z}$ are replaced by even larger circles (lower order of generation).

Having examined the way in which the radii decrease as a function of the order, $k$, we are now able to formulate an analytical expression for the distribution, $\mathrm{n}(\mathrm{r})$.

For any given first generation of circles, $\left(r_{1}, r_{2}, r_{3}\right)$, the radius of second generation circles, $\mathbf{r}_{\mathbf{4}}$, can be calculated exactly from Eq. (2). We postulate that the third generation can be derived by multiplying $\mathbf{r}_{4}$ by $c(i)$, where $c(i)$ can take on the values given in Eqs. (4), i. e.

$$
\begin{aligned}
& c(1)=0.3460 \\
& c(2)=0.3766 \\
& c(3)=0.5060
\end{aligned}
$$

This process can be repeated from generation to generation so that all circles of the kth generation, $r_{(k)}$, can be written in the general form

$$
r_{(k)}=r_{4} c\left(i_{2}\right) c\left(i_{z}\right) c\left(i_{3}\right) \ldots . c\left(i_{k-3}\right)
$$


where

$$
i_{1}, i_{2}, \ldots i_{k-2}=1,2,3
$$

By varying $i$, all the possible values of $r_{(k)}$ in one generation can be obtained. Since c(i) can assume only the above three values, we may write

$$
r_{(k)}(\alpha, \beta, \gamma)=r_{4} c(1)^{\alpha} c(2)^{\beta} c(3)^{\gamma}
$$

where $\alpha, \beta$, and $\gamma$ are linked by the relation

$$
\alpha+\beta+\gamma=k-2
$$

The multiplicity of any specific $r_{(k)}(\alpha, \beta, \gamma)$ is given by the permutation

$$
P(\alpha, \beta, \gamma)=\frac{(k-2) !}{\alpha ! \beta ! \gamma !}
$$

Equation (9) describes the probability distribution of the kth order circles, and together with Eq. (7) is the sought-for analytic expression.

The change of the mean of the logarithms of the radii of all circles of one generation, $\left\langle\ln \mathrm{r}_{(\mathrm{k})}\right\rangle$, as a function of the order of the generation, $\mathrm{k}$, is easily calculated. According to the above derivation we find

$$
\frac{\mathrm{d}\left\langle\ln \mathrm{r}_{(\mathrm{k})}\right\rangle}{\mathrm{dk}}=\frac{1}{3} \ln [\mathrm{c}(1) \mathrm{c}(2) \mathrm{c}(3)]=0.9044
$$

As a first approximation this value agrees very well with the value of 0.9075 determined from Fig. 6 .

The distribution represented by the analytic expression, Eq. and (9), is compared with two actual distributions of the same mean in Fig. 8 (for regular and irregular interstices respectively). The analytical distribution is in fair accord with the actual distributions. The large spike 


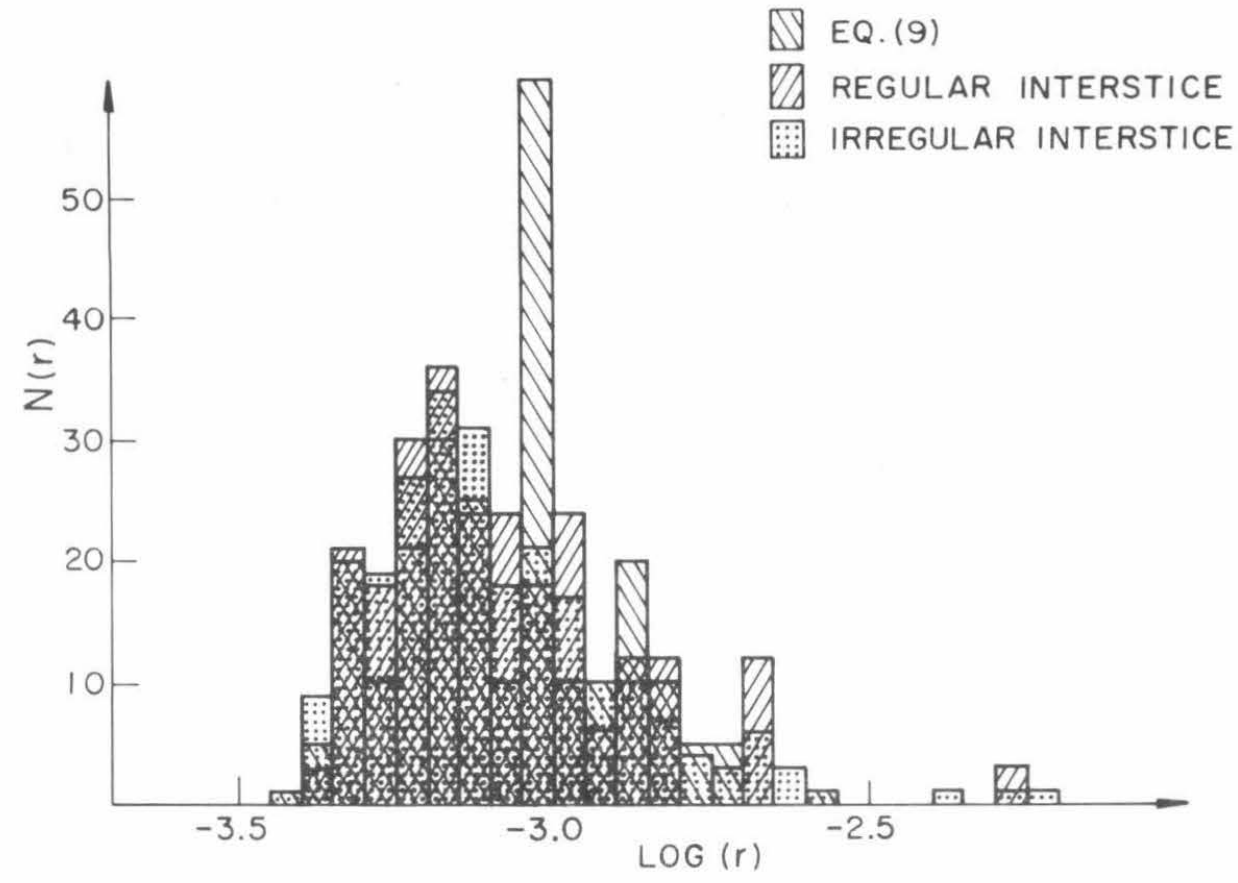

Fig. 8. Distribution of 7 th Order Circles.

is a consequence of the use of only three fixed values of c (Eqs. (5)) in the permutation. In the actual distributions, for which the values of c vary slightly, the spike will be suppressed. This is underlined by the fact that the intensity in both intervals adjoining the maximum of the analytic distribution is very small. The average over all three intervals conforms to the values obtained for the actual distributions.

For an interstice of either actual distribution an analytical expression can be derived for the cumulative distribution of radii up to the kth generation from the knowledge that the number of circles within the $j$ th generation is equal to $3^{j-2}(j \geq 2)$, and that

$$
\left\langle\ln r_{(j)}\right\rangle=\ln r_{4}-0.9044(j-2) \quad(j \geq 2) .
$$

We assume that within the jth generation half of the radii are smaller than 
$\left\langle\ell n r_{(j)}\right\rangle$. Then for all points $\ln r=\left\langle\ell n r_{(j)}\right\rangle$ the total number of circles with radii between $r$ and $r_{4}$ will be exactly

$$
Z(j)=1+3+3^{2}+\cdots \cdot 3^{j-3}+\frac{1}{2} \cdot 3^{j-2}
$$

Using the summation formula of a geometric series and expressing $j$ by ln $r$ (Eq. (11)) we obtain:

$$
Z(\ln r)=3^{-\frac{\ln r / r_{4}}{0.9044}}-0.5
$$

Equation (13) describes correctly the changes of $\mathrm{Z}\left(\ell_{\mathrm{n}} \mathrm{r}\right.$ ) but assigns too small a value to $\mathrm{Z}\left(\ell_{\mathrm{n}} \mathbf{r}_{4}\right)$. This can be compensated for by a correction factor $b . Z^{\prime}=b Z$, then, is that cumulative distribution of circles with radii $\mathbf{r}=\mathbf{r}_{\mathbf{4}}$ which identically fulfills the condition

$$
\int_{0}^{r_{4}} \frac{d z^{\prime}}{d r} \pi r^{2} d r=B
$$

where $B$ is the area of one interstice. Since B is easily obtained from geometry, b can be calculated from Eq. (14). For a regular interstice $\mathrm{b}$ is 1.385 . The normalized intensity function $\mathrm{n}(\mathrm{r})$ is derived from $\mathrm{Z}^{\prime}$ and from the number of interstices per unit area.

For regular interstices:

$$
\begin{aligned}
\mathrm{n}(\mathrm{r}) \mathrm{dr}=\frac{\mathrm{dz}}{\mathrm{dr}} \cdot \frac{0.5774}{\mathrm{r}_{1}^{2} \mathrm{dr}} & =1.683 \cdot 3^{-\frac{\ln \mathrm{r} / \mathrm{r}_{4}}{0.9044}} \frac{\mathrm{dr}}{\mathrm{r}} \\
& =1.683 \cdot\left(\mathrm{r} / \mathrm{r}_{4}\right)^{-2.215} \mathrm{dr} / \mathrm{r}_{4}
\end{aligned}
$$


For irregular interstices, i. e. for first order circles of three different diameters (cf. Fig. 2), the normalized distribution of smaller circles becomes

$$
\mathrm{n}(\mathrm{r}) \mathrm{dr}=1.706 \cdot\left(\mathrm{r} / \mathrm{r}_{4}\right)^{-2.215} \mathrm{dr} / \mathrm{r}_{4}
$$

Equations (15) and (16) differ only in the numerical front factor. This is due to the fact that the packing fractions obtained from packing of first order circles are different in the two cases.

\section{4 Random Packing}

In the previous section multimodal distributions of spheres or circles were discussed which could be realized physically only by careful handpacking. In practice the particles must occupy random sites. The random packing densities of monodisperse, hard inactive spheres seem to fall between two limits depending upon the way in which the random packing was achieved. Loose random packing $(\varphi=0.59-0.60)$ is the result of gently pouring particles on top of each other. Dense random packing $(\varphi=0.63-0.65)$ constitutes the upper limit obtained after the careful tapping and shaking of loosely packed random arrays. A theoretical prediction of random packing fractions for uniform spheres was obtained by Blum(15), and by Blum and Wilhelm ${ }^{(7)}$.

In random packing of multimodal distributions no size component will form regular lattices, but the smaller sized particles will often, though not always, take their random sites in places which are too small for larger particles. Thus the packing fraction of multimodal distributions is increased. Despite this fundamental difference in the building up of 
random and regular structures there is some agreement between optimal data calculated for regular lattices and those observed experimentally for random assemblies of particles. The information on the optimal distribution of ordered circles developed here will be applied to randomly packed spheres in the next section.

\section{Two- and Three-Dimensional Particle Size Distributions}

In the previous section it was pointed out that in a random distribution the area fraction of circles in a cross-section is equal to the volume fraction of the associated distribution of spheres. It is also desirable, furthermore, to deduce the distribution of sphere sizes, $N(R)$, from the distribution of circle sizes, $\mathrm{n}(\mathrm{r})$, within a cross-section. This problem was first treated mathematically by Wicksell ${ }^{(16)}$ in a study of the distribution of sizes of spherical corpuscles in different human organs. The normalized transformation from $N(R)$ to $n(r)$ can be derived directly from geometry. Larger spheres are more likely to be cut by a random crosssection through the sample. So the probability that a circle in this crosssection belongs to a sphere whose radius $R$ lies between $R$ and $R+d R$ is $R N(R) d R / R_{0}$ where $R_{0}$ is the average sphere radius:

$$
R_{0}=\int_{0}^{\infty} R N(R) d R
$$

The radii of circles which belong to a sphere with radius $R$ are distributed according to

$$
n_{R}(r) d r=\frac{r d r}{R \sqrt{R^{2}-r^{2}}}
$$


The total distribution of circles of radii $r$ in a cross-section is determined by contributions from all spheres with radius $R \geq \mathbf{r}$ :

$$
n(r) d r=d r \int_{r}^{\infty} n_{R}(r) R N(R) d r / R_{0}=r d r \int_{r}^{\infty} \frac{N(R) d R}{R_{0} \sqrt{R^{2}-r^{2}}}
$$

Due to the singularity at $R=r$ in the integral equation (19) no general solution for $N(R)$ as a function of $n(r)$ can be given. Goldsmith ${ }^{(17)}$ has applied a method of successive approximations and a method of iterated kernels to solve Eq. (19), but this still involves heavy use of numerical calculations. Some difficulties of the numerical calculations can be avoided if the transformation of histograms rather than that of continuous functions $n(r)$ and $N(R)$ is considered. Wicksell as well as Goldsmith have given a solution for the transformation of histograms representing equal intervals $\Delta \mathrm{r}$. It is clear from the previous section, however, that in the present problem the distribution of circle radii in intervals, $\Delta r / r$, which form a geometric progression, is important. Therefore a method of transformation of histograms with such intervals is needed. To develop this method it is necessary to study the transformation of three step-functions $N_{i}(R)$ with known properties into which any function $N(R)$ can be decomposed.

The first step-function is a rectangular distribution, $N_{I I}(R)$ in Fig. 9, which is constant between the lower limit $R_{z}$ and the upper limit $R_{1}$. The intensity can be calculated from the normalization condition

$$
\int_{R_{2}}^{R_{1}} N(R) d R=1
$$


and we obtain

$$
\mathrm{N}(\mathrm{R}) \mathrm{dR}=\frac{\mathrm{dR}}{\mathrm{R}_{1}-\mathrm{R}_{2}} . \quad \mathrm{R}_{2} \leq \mathrm{R} \leq \mathrm{R}_{1}
$$

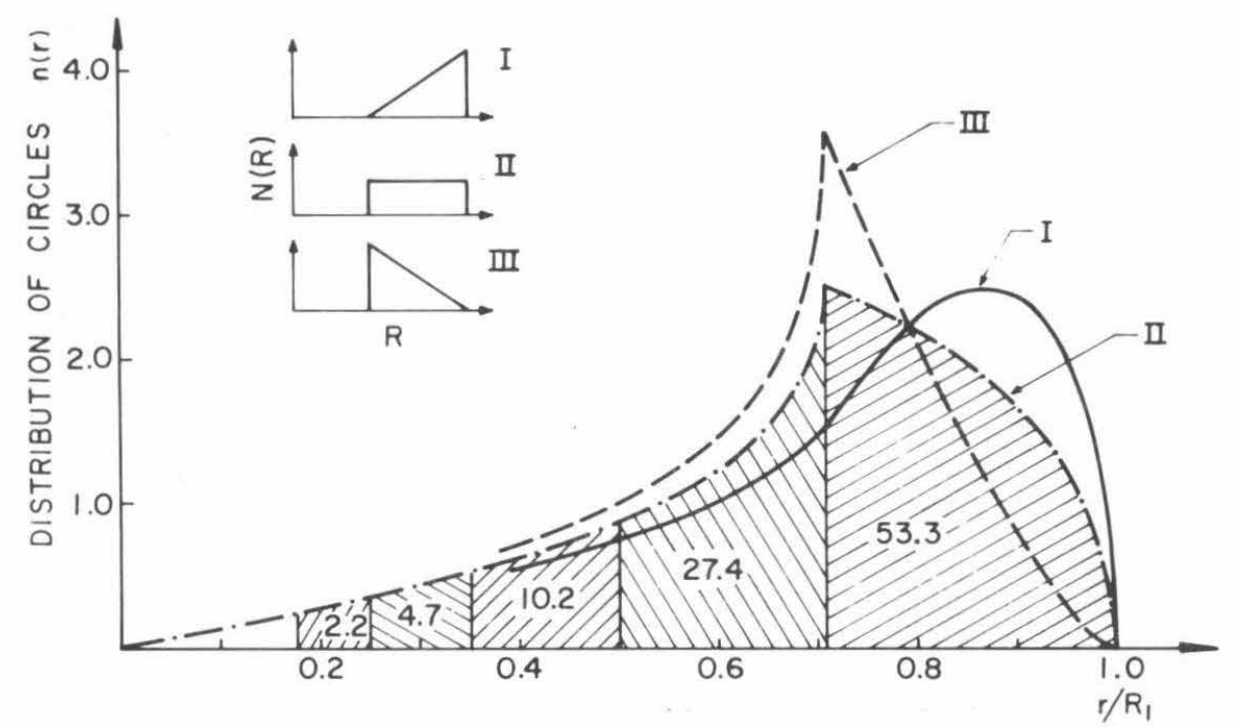

Fig. 9. Distribution of Circle Radii for Three Different Step-distributions of Sphere Radii.

If we introduce Eq. (21) into Eq. (19) we get

$$
n(r) d r=\frac{r d r}{R_{0}\left(R_{1}-R_{2}\right)} \int_{S}^{R_{1}} \frac{d R}{\sqrt{R^{2}-r^{2}}}
$$

The quantity $S$ as the lower limit of integration has to be understood as equal to $\mathbf{r}$ or $R_{2}$, whichever is larger. This follows from the restriction in Eq. (21). Integration of Eqs. (17) and (22) leads to:

$$
\mathrm{n}(\mathrm{r}) \mathrm{dr}=\frac{2 \mathrm{rdr}}{\mathrm{R}_{1}{ }^{2}-\mathrm{R}_{2}{ }^{2}}\left(\cosh ^{-1} \frac{\mathrm{R}_{1}}{\mathrm{r}}-\cosh ^{-1} \frac{\mathrm{S}}{\mathrm{r}}\right)
$$

The distribution of circle radii, $n(r)$, is normalized so that 


$$
\int_{0}^{R_{1}} n(r) d r=1
$$

The arithmetic average of $r, r_{0}$, in case of a rectangular distribution of $\mathrm{R}$ is:

$$
r_{0}=\int_{0}^{R_{1}} r n(r) d r=\frac{\pi}{12} \frac{R_{1}^{2}+R_{1} R_{2}+R_{2}^{2}}{R_{0}}
$$

The distribution of circle radii, $\mathrm{n}_{I I}(\mathrm{r})$, as derived from Eq. (23) is shown in Fig. 9. An interval of width $R_{1} / R_{2}=\sqrt{2}$ has been used in drawing Fig. 9 and in subsequent calculations. The value of $\sqrt{2}$ was selected with further application in view because $\sqrt{2}$ is commonly the ratio of diameters of distinguishable sieving fractions. It is easily understood that $\mathrm{n}_{\mathrm{II}}(\mathrm{x})$ has a maximum at $r=R_{2}=0.71 R_{1}$. At this point all spheres of the rectangular distribution contribute towards $\mathrm{n}(\mathrm{r})$. These contributions decrease at smaller values of $r$ proportional to $r$ and at larger values of $r$ proportional to $\cosh ^{-1}\left(R_{1} / r\right)$.

A better representation of a continuous distribution may be expected from trapezoidal step distributions. The largest differences between trapezoidal and rectangular step distributions occur if the former are degenerated into triangular ones. In Fig. 9 the triangular distributions $\mathrm{N}_{I}(\mathrm{R})$ with a maximum at $\mathrm{R}_{1}$ and $\mathrm{N}_{I I I}(\mathrm{R})$ with a maximum at $\mathrm{R}_{2}$ are shown together with their respective circle distributions, $n(r)$. As expected the maximum of $\mathrm{n}_{\text {III }}(\mathrm{r})$ is more pronounced than that of $\mathrm{n}_{I I}(\mathrm{r})$ and the maximum of $\mathrm{n}_{\mathrm{I}}(\mathrm{r})$ is shifted towards the upper limit. In general the behavior of $\mathrm{n}(\mathrm{r})$ is not too different in these three cases. 
Also shown in Fig. 9 are the intervals with the progression of $\sqrt{2}$. The shaded portions are equal to the intensity with which radii $\mathrm{r}$ appear within each interval. The exact value of the intensity in percent of the number of all radii is inscribed into each interval. This information is summarized in Table IV.

\section{TABLE IV}

\section{Cumulative Distribution of Circle Radii}

(for homogeneous distribution of sphere radii $R$ between $R_{1}$ and $R_{z}$ )

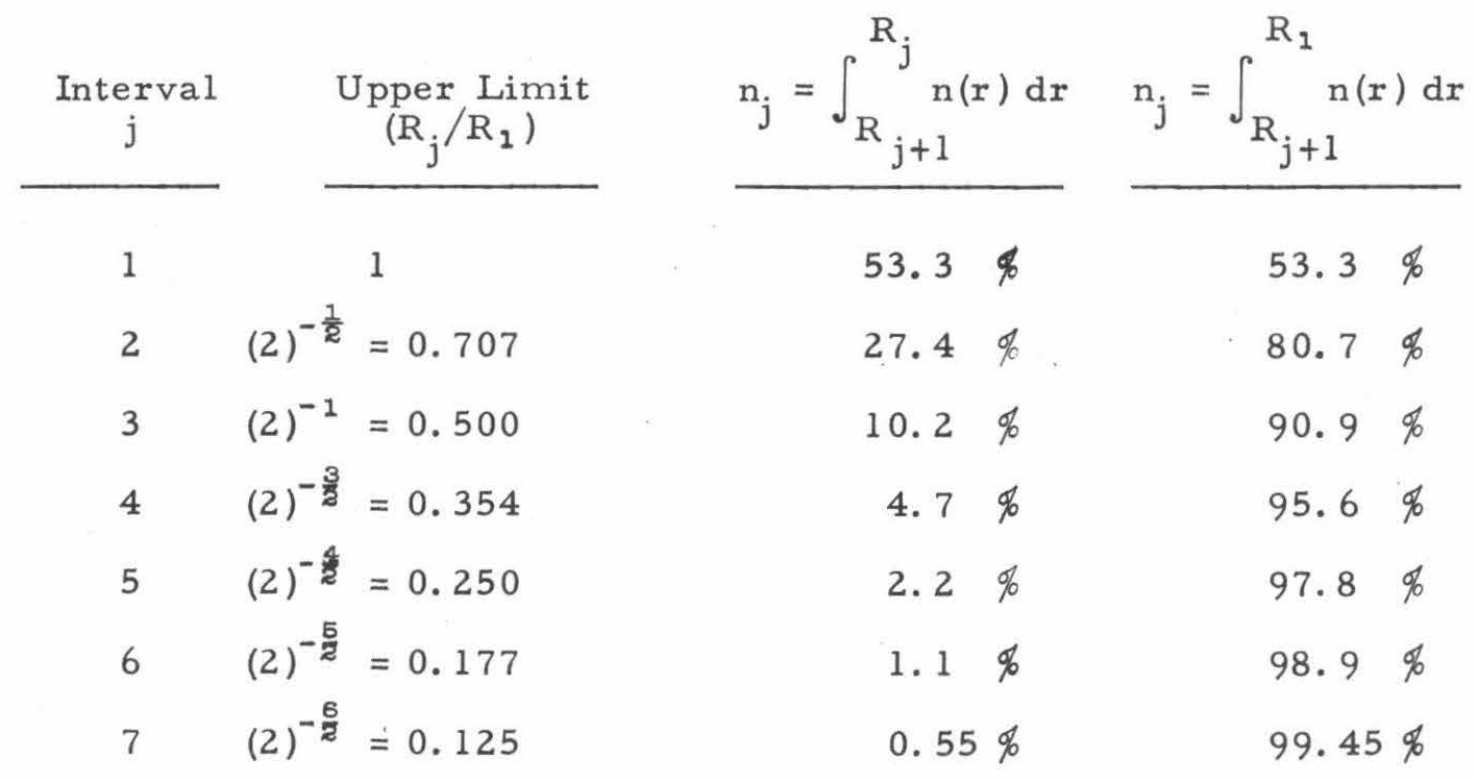

The number $j$ of the interval and the upper limit $R_{j}=2^{-(j-1) / a}$ are given in the first two columns. The intensity $n_{j}$ and the cumulative intensity are shown in columns 3 and 4 . It is seen from this table that more than $90 \%$ of all circles have radil between $0.35 R_{1}$ and $R_{1}$. This property of $n(r)$ will be used in a readily converging approximation.

We denote by $N_{j}$ the intensity of spheres with radii within the $j$ th interval. The $\mathrm{N}_{\mathrm{j}}{ }^{\prime}$ s are normalized so that 


$$
\sum_{j=1}^{\infty} N_{j}=1
$$

The presence of $\mathrm{N}_{\mathrm{k}}$ spheres with radii within a particular interval $\mathrm{k}$ will on the average produce $0.533 \mathrm{~N}_{\mathrm{k}}$ circles with radii in the $\mathrm{kth}$ interval, $0.274 \mathrm{~N}_{\mathrm{k}}$ circles in the $(\mathrm{k}+1)$ st interval and so on. If $\mathrm{N}(\mathrm{R})$ extends over many intervals the contribution of each $N_{j}$ to eacn $n_{j}$ can easily be derived in the same way. It is also clear that a particular $\mathrm{n}_{\mathrm{j}}$ receives contributions only from $\mathrm{N}_{i}{ }^{\prime} \mathrm{s}$ with $\mathrm{i}<\mathrm{j}$, i. e. the spheres must have radii which are equal or larger than the radii of the circles from which they derive. Fig. 9 shows that the $\mathrm{N}_{1}$ spheres in the first interval contribute significantly only to the intensity of circles within the first 6 intervals. The matrix for the transformation of $N(R)$ into $n(r)$, therefore, will be a triangular matrix with non-zero elements only in a band of 6 diagonals. The matrix for the inverse operation, $\mathrm{n}(\mathrm{r}) \rightarrow \mathrm{N}(\mathrm{R})$, is given by

$$
N_{i}=M_{i j} n_{j}
$$

where

$$
M_{i j}=\left[\begin{array}{cccccccc}
1.8767 & -0.9662 & 0.1386 & -0.0508 & 0.0052 & -0.0032 & 0.0000 & \ldots \\
0 & 1.8767 & -0.9662 & 0.1386 & -0.0508 & 0.0052 & -0.0032 & \ldots \\
0 & 0 & 1.8767 & -0.9662 & 0.1386 & -0.0508 & 0.0052 & \ldots \\
0 & 0 & 0 & 1.8767 & -0.9662 & 0.1386 & -0.0508 & \ldots \\
0 & 0 & 0 & 0 & 1.8767 & -0.9662 & 0.1386 & \ldots \\
\ldots & \ldots & \ldots & \ldots & \ldots & \ldots & \ldots & \ldots
\end{array}\right]
$$


An error analysis of this method leads to adequate results. If the $\mathrm{n}_{\mathrm{j}}$ are determined from experiment with error $\Delta \mathrm{n}_{j}$, the error $\Delta \mathrm{N}_{\mathrm{j}}$ of $\mathrm{N}_{\mathrm{j}}$ is predominantly determined by $n_{j}$ and about twice as large. If $\Delta n_{j} / n_{j}$ is about $5 \%$ the error $\Delta N_{j} / N_{j}$ may be as sumed to be $10 \%$.

It is of interest to know whether an error of this magnitude allows one to distinguish between distributions of increasing, constant, or decreasing slope as shown in the insert of Fig. 9. The equation for $N(R)$ can in each case be determined from the normalization condition and the known facts that $N_{I}\left(R_{2}\right)=0, N_{I I}(R)=$ constant, and $N_{I I I}\left(R_{1}\right)=0$. In each case $N(R)$ is limited to the interval from $R_{2}=\sqrt[1]{2}$ to $R_{1}=1$.

$$
\begin{aligned}
& \mathrm{N}_{I}(\mathrm{R})=16.49\left(\sqrt{2} \mathrm{R} / \mathrm{R}_{1}-1\right) \\
& \mathrm{N}_{\text {II }}(\mathrm{R})=3.414 \\
& \mathrm{~N}_{\text {III }}(\mathrm{R})=23.31\left(1-\mathrm{R} / \mathrm{R}_{1}\right)
\end{aligned}
$$

If we know that the number of spheres which have radii in the first interval is $\mathrm{N}_{\mathrm{x}} \pm 10 \%$ and if we know their distribution within the first interval, we can calculate the expected intensity $\mathrm{n}_{1}$ of circles as before. We have

$$
\begin{aligned}
& \mathrm{n}_{1}(\mathrm{I})=0.61\left(\mathrm{~N}_{\mathrm{x}} \pm 10 \%\right) \\
& \mathrm{n}_{1 \text { (II) }}=0.53\left(\mathrm{~N}_{\mathrm{x}} \pm 10 \%\right) \\
& \mathrm{n}_{1 \text { (III) }}=0.45\left(\mathrm{~N}_{\mathrm{x}} \pm 10 \%\right)
\end{aligned}
$$

An error of $10 \%$ in $\mathrm{N}_{\mathrm{x}}$ leads to the same uncertainty in $\mathrm{n}_{1}$ as the approxi- 
mation of a triangular (I or III) through a rectangular distribution (II) does. We therefore conclude that the much less pronounced differences between rectangular and trapezoidal distributions cannot be readily analyzed if the error $\Delta N_{j} / N_{j}$ is of the order of $10 \%$.

With experimental results of this accuracy the approach through a stepwise constant distribution $N(R)$ seems not only to be justified but also to be the limit of information generally obtainable. * Distributions of circles in cross-sections cut through random packings have been studied by Debbas and Rumpf ${ }^{(18)}$ to check the randomness of packings under the influence of gravity. Goldsmith ${ }^{(17)}$ also studied the influence of finite slice thickness.

Equation (27) has been applied to an actual normalized circle distribution $\mathrm{n}(\mathrm{r})$ as obtained for optimal filling of an irregular interstice (Fig. 4). The distributions $\mathrm{n}(\mathrm{r})$ and $\mathrm{N}(\mathrm{R})$ are shown in Fig. 10. The negative portion of $N(R)$ is due to the truncation of $n(r)$ at the lower limit. If Eq. (27) would be used throughout, similar negative portions of $N(R)$ would appear in the intervals $2,4,6$ to 8 , and 10 in which $\mathrm{n}(\mathrm{r})$ is zero. In order to avoid the physically senseless negative portions of $N(R)$ in these intervals a correction had to be applied. If $\mathrm{n}$ is interpreted as the number of circle radii found in the first two intervals (which it certainly is in this case), $\mathrm{N}_{1}$ can be calculated accordingly from Eq. (23). $\mathrm{N}_{z}$ will be put to zero. The intensity $\mathrm{n}_{3}$ again has to be interpreted as the total intensity of the intervals 3 and $4, n_{5}$ as the intensity of intervals 5 to $8, n_{9}$ as that of intervals 9 and

* Once $N(R)$ is obtained in first approximation as given by Eq. (27) it may be conjectured that the rate of change of $N(R)$ within one interval corresponds to the change of $N(R)$ from one interval to the next. 


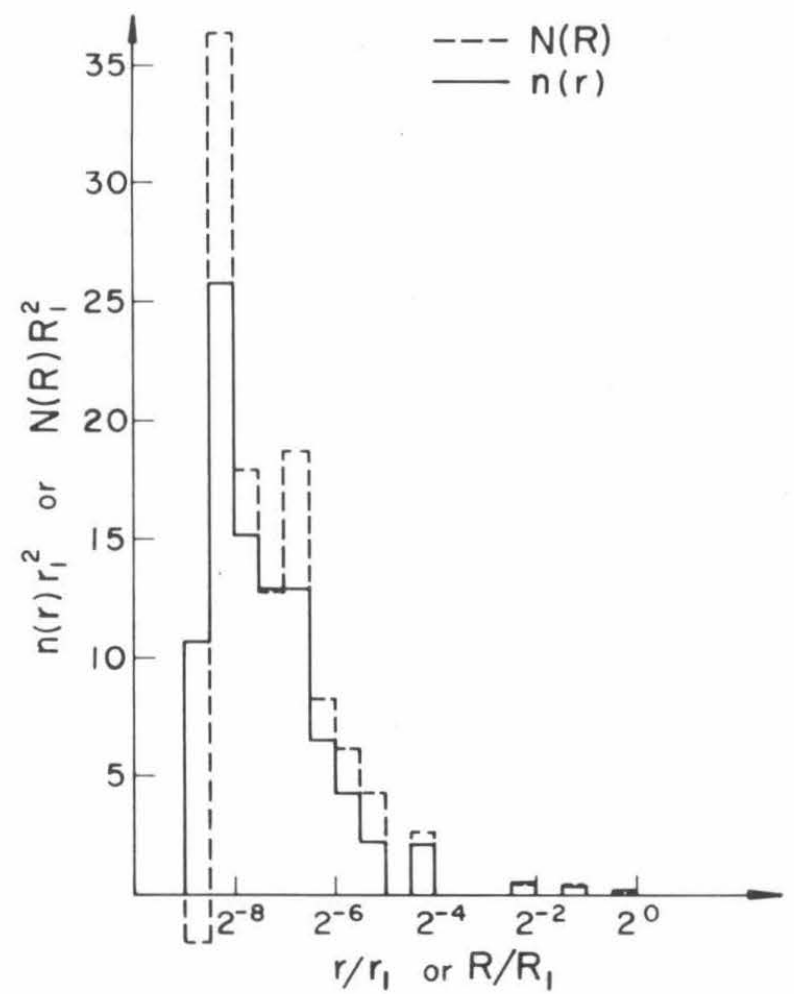

Fig. 10. Distribution of Sphere Radii Calculated from Optimal Circle Radii Distribution.

10. If this is done $N_{1}, N_{3}, N_{5}$, and $N_{9}$ can be calculated so as to give exactly that number of circles which actually appear within the first ten intervals. The $\mathrm{N}_{\mathrm{j}}$ for higher intervals are calculated from Eq. (27). 


\section{REFERENCES}

1. C. C. Furnas, Industrial and Engineering Chemistry, 23, 9, 10521058 (1931).

2. G. R. Cokelet, private communication.

3. A. E. R. Westman and H. R. Hugill, J. Am. Ceramic Soc., 13, 767 (1930).

4. F. R. Schwarzl, H. W. Bree, TNO-Nieuws, 21, 74-89 (1966).

5. J. S. Chong, Ph. D. Thesis, Department of Chemical Engineering, University of Utah (1964).

6. H. Leeming and T. W. Gillis, Bulletin of the 4th Meeting, ICRPG, Working Group on Mechanical Behavior, CPIA Pub. No. 94U, 1-28 (1965).

7. E. H. Blum and R. H. Wilhelm, AIChE-IChemE Symposium Series No. 4, (1965). (London Instn. Chem. Engrs. ), 21.

8. O. K. Rice, J. Chem. Physics, 12, 1-18 (1944).

9. G. D. Scott, Nature, 188, 908 (1960).

10. H. T. Horsfield, J. Soc. Chem. Industry, 107T-115T (1934).

11. A. Rosiwal, Verh. der k. k. geol. Reichsanstalt, 143-175 (1898).

12. H. L. Alling and W. G. Valentine, Am. J. of Science, 14, 50-65 (1927).

13. L. S. Brown and C. U. Pierson, J. of Am. Concrete Institute, $\underline{22}$, $117-123(1950)$.

14. L. Fejes Toth, Acta Mathematica Academiae Scientarum Hungaricae, 4, 103-110 (1953).

15. E. H. Blum, Ph. D. Dissertation, Department of Chemical Engineering, Princeton University (1964).

16. S. D. Wicksell, Biometrika, 17, 84 (1925).

17. P. L. Goldsmith, Brit, J. Appl. Phys., 18, 813 (1967).

18. S. Debbas and H. Rumpf, Chem. Eng. Science, 21, 583-607 (1966). 

PART III

MECHANICAL PROPERTIES OF POLYMER BLENDS

D. G. Fesko

and

N. W. Tschoegl 
PART III

MECHANICAL PROPERTIES OF POLYMER BLENDS

\section{CONTENTS}

1. Introduction. . . . . . . . . . . . . . . . III - 1

2. Polymer Blends. . . . . . . . . . . . . . III - 2

3. Block Copolymers as Blending Agents . . . . . . III - 6

4. Synthesis of Block Copolymers . . . . . . . . . III - 7

5. Research Program . . . . . . . . . . . III -10

References. . . . . . . . . . . . . . . . III 15 


\section{ILLUSTRATIONS}

Fig. 1. Ternary Phase Diagram of PolystyrenePolyisoprene-Poly(styrene-b-isoprene) System . . III - 6

Fig. 2. Schematic of Vacuum Line for Polymerization by "Living" Anion Technique . . . . . . . . III - 11

Fig. 3. Schematic Illustrating the Principle of the Melabs Rheometer. 


\section{Introduction}

Just as alloying serves as a powerful method for modifying the properties of metals, blending can be used to produce polymers which have properties different from the individual constituents. In principle, therefore, blending could be the simplest method of tailormaking propellant binders. In practice, however, it is difficult to blend polymers in a straightforward manner because of the general incompatibility of long chain molecules.

Recently, however, indications have appeared in the literature that block copolymers may be useful as blending agents and some work of a qualitative nature has been initiated in this direction $(1,2,3)$. The effect of a simple diblock copolymer in the blending together of two different homopolymers can be visualized by comparing it with the stabilization of an oil-water emulsion by a soap. A soap has a hydrophilic and a hydrophobic end and therefore is capable of providing a link between the oil and the water. A diblock copolymer is a single molecule in which two different polymer chains are covalently linked at one end. Instead of the ends being hydrophilic and hydrophobic, however, each end will be compatible with the homopolymer of its own kind, thus providing a means of stabilizing a dispersion of two polymers in each other. This technique, if successful, could lead to novel ways of formulating propellant binders.

This report describes a research program on the effect of diblock copolymers as blending agents, and on the mechanical and ultimate properties of the resulting blends. As explained in Section 2, it is anticipated that this research will also contribute to our understanding of the reinforcement process in elastomers. 
Some general characteristics of polymer blends will be reviewed, and the role of block copolymers as blending agents will be discussed in Sections 2 and 3. The synthesis of block copolymers is outlined in Section 4, and the research program is summarized in Section 5.

\section{Polymer Blends}

It is usually difficult to achieve satisfactory blending of two different polymers since they are generally incompatible with each other and tend to separate into two distinct phases.

The thermodynamics of polymer blends has received the attention of various workers $(4,5,6)$. Although no completely satisfactory theory has emerged, blending behavior is well understood qualitatively. The conditions for phase separation can be described thermodynamically by examining the equation for the free enthalpy of mixing at constant temperature

$$
\Delta \mathrm{G}_{\mathrm{m}}=\Delta \mathrm{H}_{\mathrm{m}}-\mathrm{T} \Delta \mathrm{S}_{\mathrm{m}}
$$

If the free enthalpy of mixing, $\Delta G_{m}$, is positive, mixing will not take place. The enthalpy of mixing, $\Delta \mathrm{H}_{\mathrm{m}}$, for non-polar molecules is usually estimated by the Hildebrand-Van Laar expression

$$
\Delta \mathrm{H}_{\mathrm{m}} \cong \mathrm{V}\left(\delta_{1}-\delta_{2}\right)^{2} \varphi_{1} \varphi_{2}
$$

where $\mathrm{V}$ is the volume of the solution of polymer 1 and polymer 2 and $\delta_{i}$ and $\varphi_{i}$ are the solubility parameter and volume fraction of species $i$. Thus the enthalpy of mixing is predicted to be positive. The entropy of mixing, $\Delta \mathrm{S}_{\mathrm{m}}$, is always positive but is necessarily small for polymers 
since the large size of the molecules greatly restricts the number of configurations available to the system. Therefore, even a small positive heat of mixing will result in a positive free enthalpy and, hence, in phase separation. In fact, compatibility has only been observed in mixtures of strongly interacting polymers ${ }^{(6)}$ to which Eq. (2) does not apply.

Surprisingly small differences in the structure of two polymers are sufficient to cause phase separation. Molau (7), experimenting with random styrene-acrylonitrile copolymers, observed phase separation in mixtures of two copolymers when their acrylonitrile content differed by as little as 4. 5\%. The critical difference in acrylonitrile concentration increased as solvent was added.

Polymer incompatibility, while limiting the development of polymer blends, has not been totally restrictive in the usefulness of blended products. Indeed, a number of blended materials rely on this incompatibility for their unique properties. The high impact resins are one example. They consist primarily of acrylonitrile-butadiene-styrene (ABS) plastics and rubber-modified polystyrene. In making ABS, a rubbery polybutadiene latex is added to the reaction vessel in which the emulsion copolymerization of acrylonitrile and styrene is carried out. Styrene-acrylonitrile copolymer is formed, some of which is grafted to the unsaturated sites on the butadiene particles. These grafts seem to provide a shell which is compatible with the styrene-acrylonitrile phase so that the rubbery butadiene particles remain well dispersed throughout it. These particles can readily be seen in electron photomicrographs in which osmium tetroxide has been used to stain the butadiene to provide contrast $(8,9)$. Highimpact (HI) polystyrene is a similar product in which rubber particles are 
suspended in the polystyrene matrix. These products, ABS and HI polystyrene, have in turn been used to modify other materials; for instance, ABS has been combined with polyvinyl chloride in efforts to raise the impact resistance of the latter. This procedure works to some extent, but the resulting structure has not been examined.

Closely related materials which only recently have become available are the thermoplastic elastomers. These are composed of $\mathrm{B}-\mathrm{A}-\mathrm{B}$ type block copolymers, or triblocks, in which B represents a glassy polymer chain and $A$ a rubbery polymer chain which are linked into one linear molecule. The commercially available thermoplastic elastomers consist of polystyrene-polybutadiene-polystyrene blocks. They are synthesized by anionic polymerization. In these materials, when the concentration of $B$ is small, the glassy end blocks form separate domains because of their incompatibility with the rubbery center block. (At high concentrations of $B, B$ forms the continuous phase and the domains consist of the rubbery segments.) At temperatures below the glass transition of the end blocks one has, therefore, a system which behaves like a dispersion of uniformly distributed hard particles in a rubbery matrix. There is essentially "perfect" adhesion between the particles and the matrix. Unpublished work of Morton ${ }^{(10)}$ and of Smith ${ }^{(1)}$, however, indicates that the ultimate properties of these materials are no different from those of a solid dispersion of styrene spheres in a poly(styreneco-butadiene) matrix without "perfect" adhesion when the size of the glassy domains in the triblocks or the size of the spheres in the dispersion are sufficiently small. 
There is at present very little information concerning the role of domain size in determining the mechanical and ultimate properties of polymer blends or block copolymers. Morton (10), however, also produced evidence that the tensile strength of his solid dispersion increased when the size of the styrene particles was decreased.

If two homopolymers are blended, the domain size depends on the materials being mixed, the relative amounts of each, and possibly the molecular weights of the materials. Marsh, et al. (12) determined the average domain size in various binary mixtures of styrene-butadiene, isoprene, butadiene, chloroprene, ethylene-propylene, and nitrile rubber. Compatibility was noted for styrene-butadiene with butadiene rubber, i.e. domains could not be distinguished, but average domain sizes ranging from 0.2 to 30 microns were observed in the other cases. The distributions of domain sizes in the mixtures were broad.

The domain size in B-A-B triblock copolymers depends on the molecular weight. For a polystyrene moiety of about 10,000 , the radius of the glassy domain is about $200 \mathrm{~A}^{(13)}$ and the distribution of domain sizes is narrow. Riess et al. (2) and Kohler ${ }^{(3)}$ have shown that clear films could be cast from a solution containing two homopolymers together with the corresponding $A-B$ diblock copolymer. It appears possible, therefore, to control the domain size of polymer blends by varying the lengths of the $\mathrm{A}$ and $\mathrm{B}$ chains in a diblock copolymer which has been added as a blending agent. 


\section{Block Copolymers as Blending Agents}

Ceresa ${ }^{(1)}$ was probably the first to observe the potential of block copolymers as blending agents. With the addition of $5 \%$ of an epoxy resinpoly(methyl methacrylate) block copolymer to a $1: 1$ solution of epoxy and poly(methyl methacrylate), he was able to cast clear films. More recently Riess et al. ${ }^{(2)}$, and Kohler ${ }^{(3)}$ have investigated ternary systems of polystyrene - poly(methyl methacrylate) and polystyrene - 1, 4-cispolyisoprene with their block, graft, and random copolymers. Using the optical clarity of films of varying composition as a qualitative measure of compatibility, they obtained ternary phase diagrams as shown in Fig. 1 for one system. Clear films resulted generally only in the case of the block

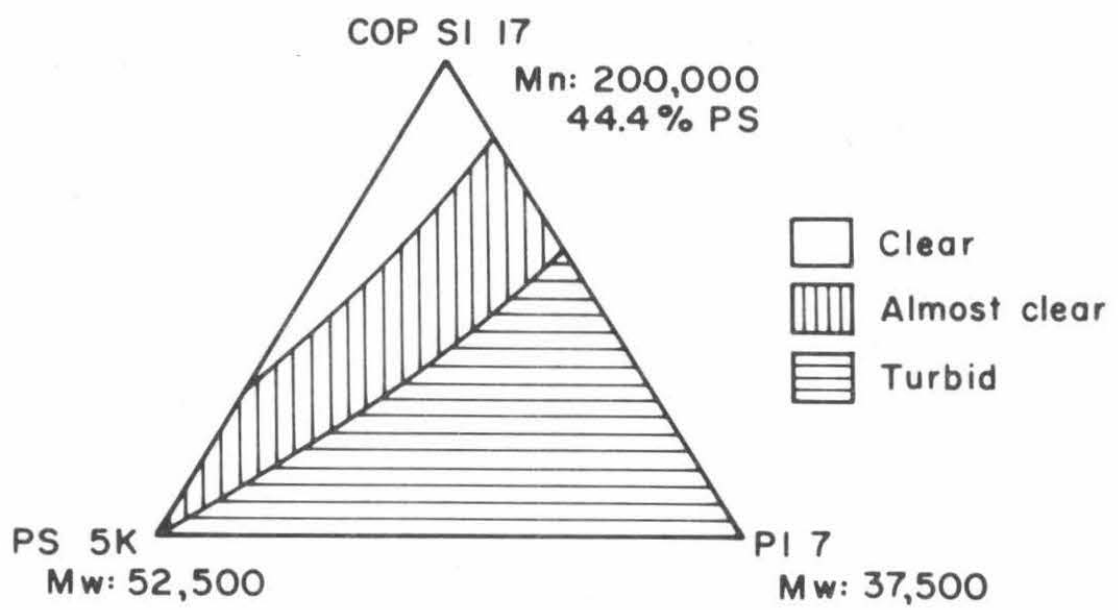

Fig. 1. Ternary Phase Diagram of Polystyrene - PolyisoprenePoly(styrene-b-isoprene) System.

copolymers, particularly when the homopolymer composition was near 50 : 50 and the molecular weights of the homopolymers were less than those of the blocks of the copolymers. Figure 1, taken from Kohler's thesis, shows a polystyrene-polyisoprene system indicating qualitatively 
three degrees of clarity. The copolymer, designated COP SI 17, had a number average molecular weight of 200,000 , being $44.4 \%$ polystyrene by weight. The polystyrene, PS $5 \mathrm{~K}$, had a weight average molecular weight of 52,500. That of the polyisoprene, PI 7, was 37,500. Some of the systems were subjected to Charpy impact tests to obtain some indication of the impact properties.

\section{Synthesis of Block Copolymers}

Block copolymers consist of different homopolymers which are linked into a linear chain. They are related to graft copolymers, which consist of polymers of one type branching off the backbone of another type. Pike and Watson ${ }^{(14)}$ showed that free radicals were formed when polymer molecules were torn apart under conditions of high shear. In a homopolymer the free radicals will either recombine, or they will be "killed" by free radical acceptors (such as oxygen) that are present as impurities. When, however, different homopolymers are milled together, recombination and chain transfer can result in the formation of more complex structures. Studies on block and graft copolymers prepared by this method were reported by Ceresa ${ }^{(1)}$. Block copolymers can also be obtained from homopolymers with reactive end groups ${ }^{(15)}$.

In the late 1950's Szwarc et al. ${ }^{(16)}$ introduced a polymerization technique that was to provide an extremely powerful new tool for polymer synthesis. This was the preparation of so-called "living" polymers by means of an anionic polymerization carried out so that no termination reactions can occur. When the monomer is used up, the polymer will stay "alive" for long periods of time and will continue to polymerize whenever more monomer is added. Continuation of the reaction by a different 
monomer leads to the formation of a block copolymer. The "living" anions are obtained through meticulous control of the experimental conditions. Any impurity that might cause termination must be carefully removed. The usual procedure is to use elaborate purification techniques and a high vacuum environment. The equipment and procedure have been described by several workers. $(17,18,19,20)$

Two types of anionic initiators can be used to begin the polymerization:

(1) initiation by electron transfer, and

(2) initiation by anion attack.

Both methods use organo-metallic compounds as initiators. Initiation by electron transfer is usually achieved by a compound such as sodium naphthalene. The valence electron of the sodium is stabilized in the $\pi$-orbitals of the naphthalene $(21,22)$. With this extra electron, the naphthalene has characteristics between those of a free radical and an anion. When initiation takes place, $(22,23,24,25)$ the extra electron is transferred to the monomer. When a second monomer unit is added, the free radical and

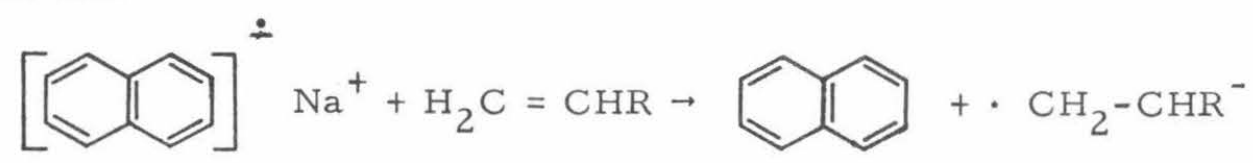

the anionic charge assume separate identities at opposite ends of the molecule, and polymerization can proceed at either end by free radical or anionic means respectively $(24,26,27)$. If the molecule comes in contact with another radical anion, the free radicals can combine leaving a chain growing at both ends by anionic addition only. When applied to block copolymerization, the reaction produces B - A - B block copolymers. 
Often, however, triblock copolymers are synthesized from diblocks through a suitable coupling reaction.

Anion attack is observed when organometallic initiators are used in which electron delocalization does not occur. Various organometallic compounds fall in this class, including Grignard reagents, but organolithiums are of most interest because of their activity and their solubility in nonpolar solvents. The initiation takes place as

$$
\mathrm{R}^{-} \mathrm{Li}^{+}+\mathrm{H}_{2} \mathrm{C}=\mathrm{CH}-\mathrm{R} \rightarrow \mathrm{R}-\mathrm{CH}_{2}-\mathrm{CH}^{-}-\mathrm{R} \mathrm{Li}^{+}
$$

Since the chains grow only at one end, the reaction leads to the formation of $\mathrm{A}-\mathrm{B}$ block copolymers.

Under certain conditions, sterospecific reactions can be carried out by these anionic polymerizations. For instance, isoprene polymerized in a nonpolar solvent with lithium compounds as initiators gives essentially all cis-1,4 product. Likewise, styrene in a nonpolar solvent below $-30^{\circ} \mathrm{C}$ will give a syndiotactic product.

Polymerization by "living" carbanions has many noteworthy features. All of the molecules grow at the same rate. Hence, if the initiation is carried out rapidly, the resulting polymer has a narrow distribution of molecular weights which has the character of a Poisson distribution $(28)$. Thus, much more precision is available in preparing laboratory samples of controlled molecular weight, as well as greatly increased flexibility in tailormaking needed polymers. Also, the absence of chain transfer and termination reactions simplifies the reaction kinetics so that they can be described more accurately. 


\section{Research Program}

The goal of this investigation is to study the mechanical properties of blends of homopolymers with the corresponding diblocks. The first phase of the program will consist in the preparation of the samples, and in the study of the effect of the relative chain lengths of the homopolymers and diblock copolymers on the size of the domains in the blends. The dynamic mechanical properties of the blends will be examined during the second phase. Particular attention will be paid to the effect of domain size on the dynamic loss. The ultimate properties of the blends will be the subject of the third phase.

The first structures will be made on uncrosslinked systems containing polymerizates of styrene on the one hand, and of 1,4-cis-isoprene, 1, 4butadiene, or 1,2-butadiene on the other. These systems were chosen

because it is known that the homopolymers are incompatible, (29) and because investigations on tri- and diblock copolymers polymerized from the above monomers are currently under way in this laboratory. Initially, isoprene, a liquid, will be favored over butadiene which is a gas and therefore a little more difficult to use. When sufficient knowledge has been gained on the basic behavior of diblock-stabilized homopolymer blends, the study will be extended to crosslinked elastomeric systems.

The samples will be prepared by anionic polymerization in a high vacuum environment to eliminate chain transfer and chain termination reactions. The apparatus for this has been built and a schematic of the vacuum line is shown in Fig. 2. The vacuum manifold has nine groundglass fittings for flasks in which the purification and reaction processes will be carried out. The three-stage mercury diffusion pump maintains a pressure below the lowest division on the McLeod Gauge $\left(10^{-5}\right.$ torr $)$. 


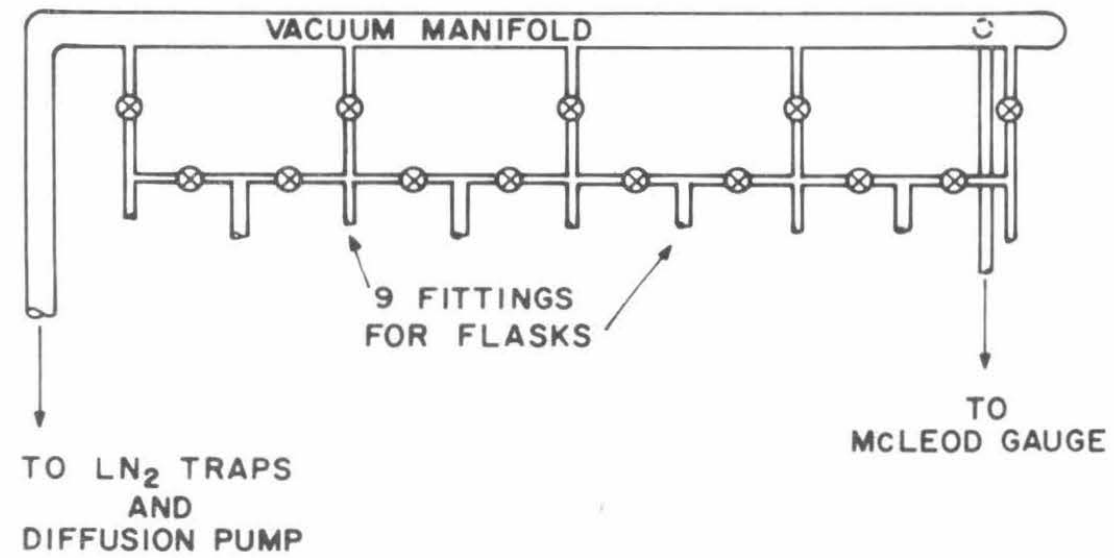

Fig. 2. Schematic of Vacuum Line for Polymerization by "Living" Anion Technique.

The initiator is restricted to alkyllithiums since these are soluble in nonpolar solvents. The particular initiator chosen for the polymerization is sec-butyllithium. This was chosen on the basis of the work of Kuntz ${ }^{(30)}$ and Hsieh ${ }^{(31)}$ because of the rapidity of initiation. In cases where initiation is slow, a "seeding" technique ${ }^{(32)}$ must be used to prepare the initiator or else a broad molecular weight distribution will result. In "seeding", small amounts of monomer are added at a time until all the initiating species are consumed. Then the rest of the monomer is added. The amount of monomer needed for seeding depends on the nature of the initiator, but it will typically be many times greater than the amount of the initiator.

By using sec-butyllithium as initiator, it should be possible to carry out the polymerization without seeding. The polystyrene initiation is slow in a nonpolar medium, but is rapid in polar solvents, and therefore, the polystyrene polymerization will be carried out in tetrahydrofuran (THF). This must be done at low temperatures since above $-35^{\circ} \mathrm{C}$ organometallic 
compounds may cleve ethers (33). Using the polar THF also results in an atactic polymer whereas a nonpolar medium may lead to an isotactic or syndiotactic micro-structure ${ }^{(34)}$.

The polyisoprene has to be prepared in a nonpolar medium such as $\mathrm{n}$-hexane in order to obtain the necessary stereospecificity. Better than 90\% cis-1,4-polyisoprene may be obtained in this manner. Stearns and Foreman ${ }^{(35)}$ suggest polymerizing at $0^{\circ} \mathrm{C}$ for best results, in which case the reaction takes several days. The initiation, however, is relatively rapid compared to the rate of propagation. The work of previous investigators $(35,36)$ indicate that these conditions lead to a narrow molecular weight distribution. This, however, bears further investigation, for it has been theorized ${ }^{(28)}$ that polymerization near equilibrium will result in the broad most probable distribution rather than the Poisson distribution. It should therefore be ascertained whether the reaction is near equilibrium at $0^{\circ} \mathrm{C}$.

In synthesizing a polystyrene-polyisoprene diblock copolymer, the isoprene can be polymerized first. To ensure rapid initiation of the second polymerization, THF can be added to the reaction mixture before addition of the styrene.

To date, only a homopolymer, a polystyrene having a molecular weight of about 128, 000, has been prepared and is being analyzed.

The molecular weights of the homopolymers will be determined by viscometry ${ }^{(37)}$ or by osmometry. The molecular weight distribution of both homopolymers and copolymers will be checked by gel permeation chromatography. The method of Kolthoff et al. (38) will probably be used to determine the molecular weight of the block copolymers. In this method 
a portion of the reaction mixture is extracted on completion of the first stage of the synthesis. Since this is a homopolymer, its molecular weight is easily determined by viscometry or osmometry. The block copolymer is formed and a portion of it is weighed and then degraded by tert-butyl hydroperoxide. All of the double bonds in the polyisoprene are broken and oxidized to yield low molecular weight aldehydes. The remaining polystyrene is precipitated in ethanol while the aldehydes remain in solution. The weight of the polystyrene remaining is determined so that the weight ratio of polystyrene to the polyisoprene and subsequently the total average molecular weight can be calculated.

Blends can not be prepared by milling for the reasons outlined in Section 4. Blended samples for examination will be cast from solution. This will also help to obtain phase separation as close to equilibrium as possible. The samples will be examined for phase separation by phase contrast microscopy and, if necessary, electron microscopy.

A Melabs Shear Rheometer is currently being evaluated for its usefulness in obtaining dynamic modulus data on the polymer blends. The design of this instrument is based on the shear generator of Miles (39). In it, as shown in Fig. 3, an oscillatory strain is imposed on a small, disk-shaped specimen by a stack of piezoelectric crystals which are excited from a function generator. The force is transmitted through the specimen, picked up by a piezoelectric transducer, and the resulting signal is amplified in a charge amplifier. The apparatus is calibrated using small steel springs of known modulus. The specimen is held in place by exerting a small lateral force on the transducer. The rheometer requires only a small specimen and has an operative temperature range from about $-200^{\circ}$ to $+250^{\circ} \mathrm{C}$. 


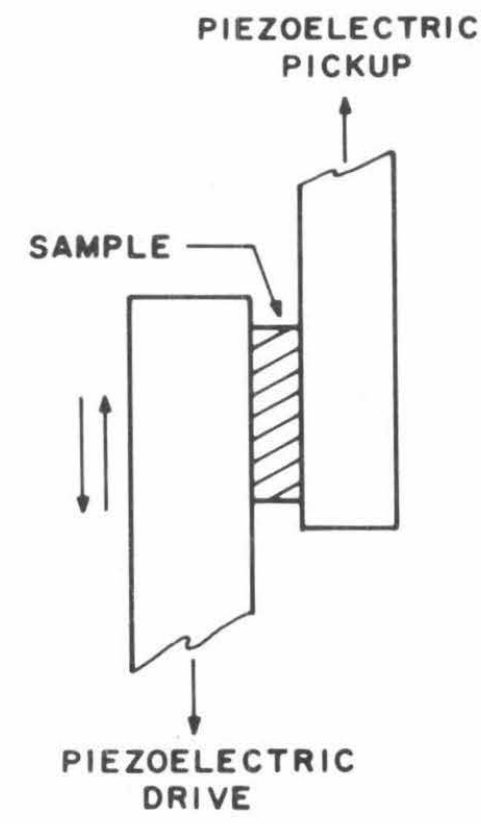

Fig. 3. Schematic Illustrating the Principle of the Melabs Rheometer.

The dynamic moduli will be obtained from the amplitude ratio and phase angle between the input and output signal. According to the manufacturers recommendations, the phase angle would be measured on a doublebeam oscilloscope. This method, however, is not very accurate with small phase angles, and is unsuitable at frequencies below about $5 \mathrm{~Hz}$. An attempt is being made to extend the frequency range of the apparatus from the present 5 to $1000 \mathrm{~Hz}$ down to about $0.1 \mathrm{~Hz}$ or lower. For this purpose the differential Lissajous method of Smith and Tschoegl ${ }^{(40)}$ will be used. This method was developed for the precise measurement of the small phase angles of propellant binders at low frequencies. The necessary electronics have been breadboarded and are currently being checked out. 


\section{REFERENCES}

1. R. Ceresa, J. Poly. Sci., 53, 9 (1961).

2. V. Riess, J. Kohler, C. Tournut, and A. Banderet, Makro. Chem., 101, 58 (1967).

3. J. Kohler, Theses, L'Universite de Strasbourg (1967).

4. R. L. Scott, J. Chem. Phys., 17, 279 (1949).

5. G. Allen, G. Gee, and J. P. Nicholson, Polymers, 1, 56 (1960).

6. P. J. Flory, Principles of Polymer Chemistry, Ithaca, New York, Cornell University Press (1953).

7. G. Molau, J. Poly. Sci., 3A, 1267 (1965).

8. K. Kato, Polymer Letters, $\underline{4}, 35$ (1966).

9. P. A. Traylor, Anal. Chem., 33, 1629 (1961).

10. M. Morton, Polymer Preprints, 으, 1569 (1967); Trans. IRI, (in press).

11. T. L. Smith, J. Polymer Sci., C, (in press).

12. P. A. Marsh, A. Voet, L. D. Price, and T. J. Mullens, Rubber Chem. and Tech., $\underline{41}, 2,344$ (1968).

13. G. Holden, E. T. Bishop, and N. R. Legg, J. Polymer Sci., C, (in press).

14. M. Pike and W. F. Watson, J. Poly. Sci., 9, 229 (1952).

15. V. Immergut, and H. Mark, Makro. Chem., 18/19, 322 (1956).

16. M. Szwarc, M. Levy, and R. Milkovich, J. Am. Chem. Soc., 78, 2656 (1956).

17. M. Morton, R. Milkovich, D. McIntyre, and L. Bradley, J. Poly. Sci., 1A, 443 (1963).

18. L. Fetters, J. of Research of the N.B.S., 70A, 5 (1966).

19. M. Morton, A. Rembaum, and J. Hall, J. Poly. Sci., 1A, 461 (1963).

20. A. Tobolsky, A. Rembaum, and A. Eisenberg, J. Poly. Sci., 4ㄴ, 347 (1960).

21. D. Paul, D. Lipkin, and S. Weissman, J. Am. Chem. Soc., 78, 116 (1956). 
22. M. Szwarc, J. Poly. Sci., 16, 339 (1963).

23. M. Levy, and M. Szwarc, J. Am. Chem. Soc., 82, 521 (1960).

24. M. Szwarc, Makro. Chem., 35, 132 (1960).

25. P. Sigwalt, Bull. Chim. Soc. France, 423 (1964).

26. K. O'Driscoll, and A. Tobolsky, J. Poly. Sci., 31, 123 (1958).

27. K. O'Driscoll, R. Boudreau, and A. Tobolsky, J. Poly. Sci., 31, 115 (1958).

28. W. B. Brown, and M. Szwarc, Trans. Faraday Soc., 54, 416 (1958).

29. R. J. Angels, R. M. Ineda, and M. L. Wollach, Polymer $\underline{6}, 141$ (1965).

30. I. Kuntz, J. Poly. Sci., 2A, 2827 (1964).

31. H. Hseih, J. Poly. Sci., 3A, 153 (1965).

32. D. Worsfold, and S. Bywater, Can. J. Chem., 38, 1891 (1960).

33. H. Gilman, B. Gaj. J. Org. Chem., 22, 1165 (1957).

34. D. Worsfold, and S. Bywater, Makro. Chem., 65, 245 (1963).

35. R. Stearns, and L. Forman, J. Poly. Sci., 41, 381 (1959).

36. S. Schlick, and M. Levy, J. Phys. Chem., 64, 883 (1960).

37. R. Waack, A. Rembaum, J. D. Coombes, and M. Szwarc, J. Am. Chem. Soc. , 79, 2026 (1957).

38. I. M. Kolthoff, T. S. Lee, and C. W. Carr, J. Poly. Sci., 1, 429 (1946).

39. D. O. Miles, J. Appl. Phys., 33, 1422 (1962).

40. N. W. Tschoegl and J. R. Smith, in: "Viscoelastic Properties of Solid Propellants and Propellant Binders, "Final Report on SRI Projects PRU-3939, 4660, 5174, Stanford Research Institute, Menlo Park, California, 1966. Department of the Navy, Bureau of Naval Weapons Contracts No. NOw 61-1057-d, NOw 64-0073-d, NOw 65-0061-d. 



\section{PART IV \\ FURTHER CHARACTERIZATION OF GALCIT I*}

R. F. Landel

* This work was carried out in collaboration with Dr. Robert F. Fedors at the Jet Propulsion Laboratory, California Institute of Technology, Pasadena, California 
PART IV

FURTHER CHARACTERIZATION OF GALCIT I

\section{CONTENTS}

1. Introduction. . . . . . . . . . . . . . . . . IV - 1

2. Determination of the Effective Chain Concentration . . IV - 3

3. Dependence of the Failure Envelope on

Prepolymer Ratio. . . . . . . . . . . . . . $\quad$ IV - 7

4. Interaction Parameters. . . . . . . . . . . . . IV - 13

References . . . . . . . . . . . . . . . IV - 18 


\section{ILLUSTRATIONS}

Fig. 1. Tensile Failure Envelopes for Solithane 113 as a Function of Composition . . . . . . . . IV - 2

Fig. 2. Dependence of Apparent Chain Concentration,

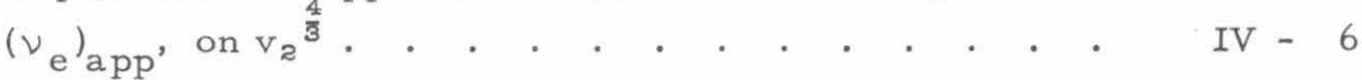

Fig. 3. Mooney-Rivlin Plot for GALCIT I at $20^{\circ} \mathrm{C}$. . . . IV - 6

Fig. 4. Failure Envelopes for the 50/50 and 55/45 Solithane Compositions . . . . . . . . . . IV - 8

Fig. 5. Sketch of the Chain Structure between Crosslinks for GALCIT I. . . . . . . . . . . . . . IV - 11

Fig. 6. Reaction of Isocyanate and Urethane Group to Form Allophanate. . . . . . . . . . IV - 12

Fig. 7. Dependence of Apparent Polymer-Solvent

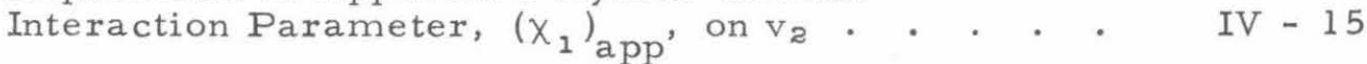

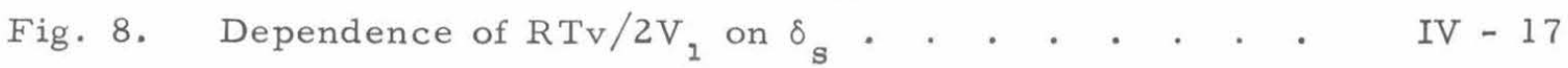




\section{TABLES}

Table I Swelling Behavior and Apparent Chain Concentration, in $10^{-6}$ moles $/ \mathrm{cm}^{3}$, for Polyurethane Elastomers.

Table II Estimates of $\nu_{e}$ and $n$ from Failure Envelopes. . . . . . . . . . . . . IV - 9

Table II Polymer-Solvent Interaction Parameters . . . . IV - I4 


\section{Introduction}

One of the goals of this research program on solid propellant

physical behavior is to prepare, initiate the characterization, and distribute for further study, two standardized rubbers. One of these should be castable, so that complex shapes can be readily prepared, and the second should be a conventional milled rubber with the advantage of ease of preparation.

Previous reports have indicated the criteria adopted for such standardized rubbers. These are briefly restated here for emphasis and clarity; more complete statements are given in earlier reports:

1) the chemical structure of the backbone and the chemistry of the crosslinking reaction should be known.

2) the crosslink density should be easily variable over wide ranges.

3) the glass transition temperature should be readily varied by changing the backbone.

4) the strain optical coefficient should be as high as possible and the specimens should be optically clear.

5) the material should be stable.

One interim standard has already been developed. This is

GALCIT I, a polyurethane obtained by reacting equal volumes of a "prepolymer" (Thiokol Solithane 113), with a "curing agent"(castor oil). Castor oil, the glyceryl ester of ricinoleic acid, is a triol, the fatty acid chains bearing hydroxyl groups on the twelfth carbon atom. Solithane 113 consists of castor oil capped with tolylene diisocyanate (TDI).

Several characterization studies have previously been carried out on GALCIT I. The present report carries on with these studies and with further analyses of earlier data. 
In the last annual report ${ }^{(1)}$, the failure envelopes of five compositions of GALCIT I, varying in prepolymer/curing agent ratio*, were presented and a preliminary analysis of these envelopes was given. The envelopes are shown in Fig. 1. The high temperature or small strain

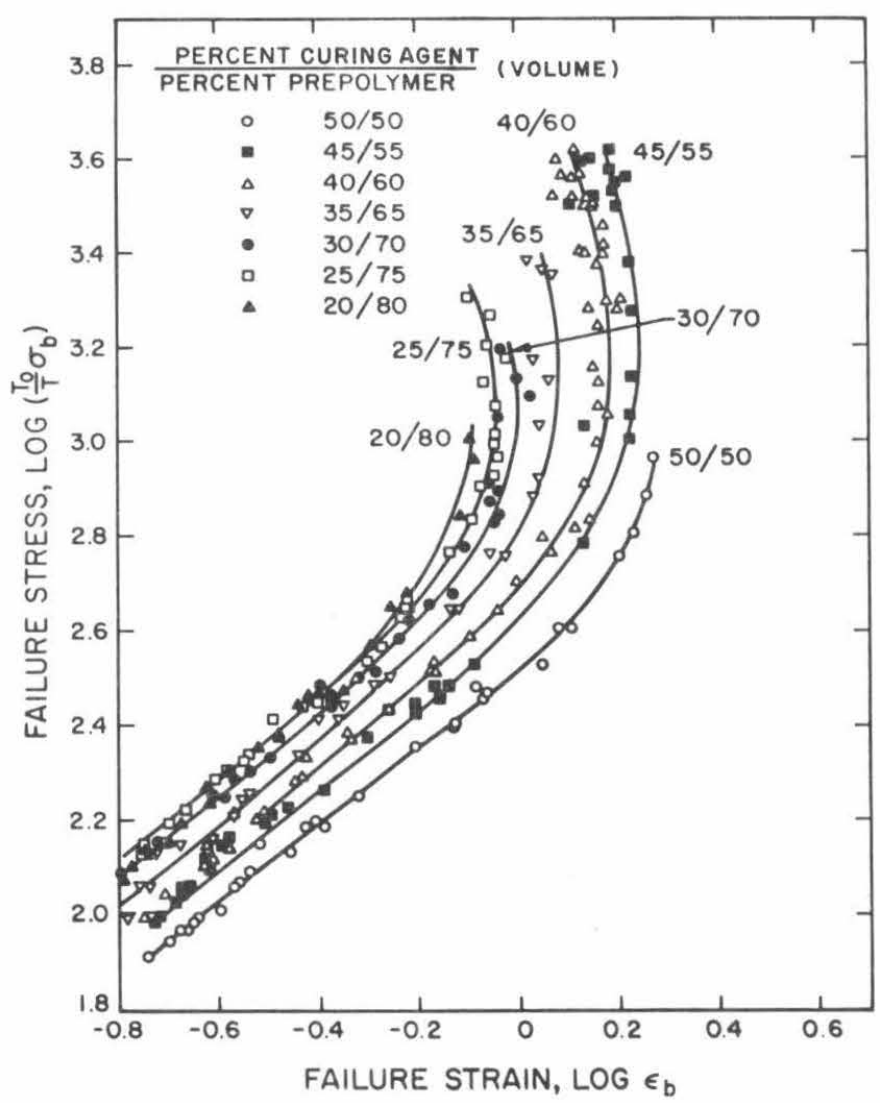

Fig. 1. Tensile Failure Envelopes for Solithane 113 as a Function of Composition.

portions of the envelopes were expected to reflect the magnitude of the crosslink density as given by the effective network chain concentration

* In the past, the curing agent has been called the catalyst, in conformance with the manufacturers designation of this second component. The material is not a catalyst, however, but enters into the reaction and so we shall henceforth use the more appropriate designation of "curing agent." 
$\nu_{e}(1,2), i . e$. at a given value of the breaking strain $\varepsilon_{b}$ the values of the breaking stress $\sigma_{\mathrm{b}}$ were expected to be proportional to $\nu_{\mathrm{e}}$. In addition, maximum $/$ tretch ratios observed, $\left(\lambda_{b}\right)_{\max }$, were expected to vary as $\nu^{-\frac{1}{2}}$. Hence $\sigma_{b}$ should have been proportional to $\left(\lambda_{b}\right)_{\max }^{-\frac{1}{2}}$. However, when the magnitude of $\sigma_{b}$ determined at $\varepsilon_{b}=25 \%$ was compared to $\left(\lambda_{b}\right)_{\text {max }}$, the results showed it to vary as $\left(\lambda_{b}\right)_{\max }^{-0.6}$ instead. Swelling experiments and compressive stress-strain measurements in the swollen state were therefore carried out to determine the sol fraction, if any, and the value of $\nu_{e}$. Only the sol fraction results were available at the time of the last report, and the sol content was found to be extremely small in all compositions.

The results of the more complete experiments and a further analysis of the failure envelopes will be presented in the following sections.

\section{Determination of the Effective Chain Concentration}

The method of estimating $\nu_{\mathrm{e}}$ from the stress-strain behavior of swollen elastomers in compression tests has already been adequately described ${ }^{(3)}$. The data were obtained on an Instron testing machine using a crosshead speed of $0.02 \mathrm{in} / \mathrm{min}$. The apparent $\nu_{\mathrm{e}}$ values were calculated by means of the following equation ${ }^{(3)}$

$$
\nu_{\mathrm{e}}=\frac{\mathrm{mh}}{3 \mathrm{ART}}=\frac{2 \mathrm{C}_{1}}{\mathrm{RT}}+\frac{2 \mathrm{C}_{2}{ }^{\prime \prime} \mathrm{v}_{2}{ }^{4 / 3}}{\mathrm{RT}}
$$

where $\mathrm{m}$ is the slope of the load deflection curve, $\mathrm{h}$ is the height and A the original undeformed area of the specimen before swelling, $R$ is the gas constant, $T$ is the absolute temperature, $v_{\boldsymbol{Z}}$ is the volume fraction of rubber in the swollen gel, $2 \mathrm{C}_{1} / \mathrm{RT}$ is the value of $\nu_{\mathrm{e}}$ at infinite swelling, and $2 \mathrm{C}_{2}$ " is related to the $2 \mathrm{C}_{2}$ term which appears in the Mooney-Rivlin (4) 
stress-strain equation. When $\mathrm{v}_{z}=1$, then $2 \mathrm{C}_{z}{ }^{\prime \prime}=2 \mathrm{C}_{2}$. It is important to notice that for $\mathrm{C}_{2}^{\prime \prime} \neq 0, \nu_{\mathrm{e}}$ will depend on $\mathrm{v}_{2}$.

The values of $\nu_{e}$ thus obtained are given in Table I, along with the extent of equilibrium swelling in five solvents: benzene, ethylene dichloride, cyclohexane, heptane, and isooctane. For a given solvent there occurs a marked and monotonic increase in $v_{z}$ as the polymer to curing agent ratio is increased. Normally, such an increase in $v_{\boldsymbol{z}}$ would be ascribed to an increase in the network chain concentration, but the results of the compression tests indicate that, within the estimated experimental error, the $\nu_{\mathrm{e}}$ values are essentially independent of the ratio of prepolymer to curing agent. Furthermore, this behavior is observed for all five solvents employed.

In addition, the data indicate that $\nu_{e}$ is almost independent of $v_{2}$, as shown in Fig. 2, where the data are plotted in accordance with Eq. (1). Hence $\mathrm{C}_{z}$ " is very nearly zero. This signifies that the stress-strain data for the dry rubber when plotted in Mooney-Rivlin coordinates should also have a slope, or $\mathrm{C}_{z}$ value,very close to zero. This is confirmed by the work of Mueller ${ }^{(5)}$, as shown in Fig. 3.

Since $\nu_{e}$ is essentially independent of the prepolymer/curing agent ratio, the fact that $v_{z}$ varies with this ratio can be taken as an implication that the chemical nature of the network chains depends on this ratio, while the number of chains does not.

Data which bear on this point will now be presented. 


\section{TABLE I}

Swelling Behavior and Apparent Chain Concentration,

in $10^{-6}$ moles $/ \mathrm{cm}^{3}$, for Polyurethane Elastomers

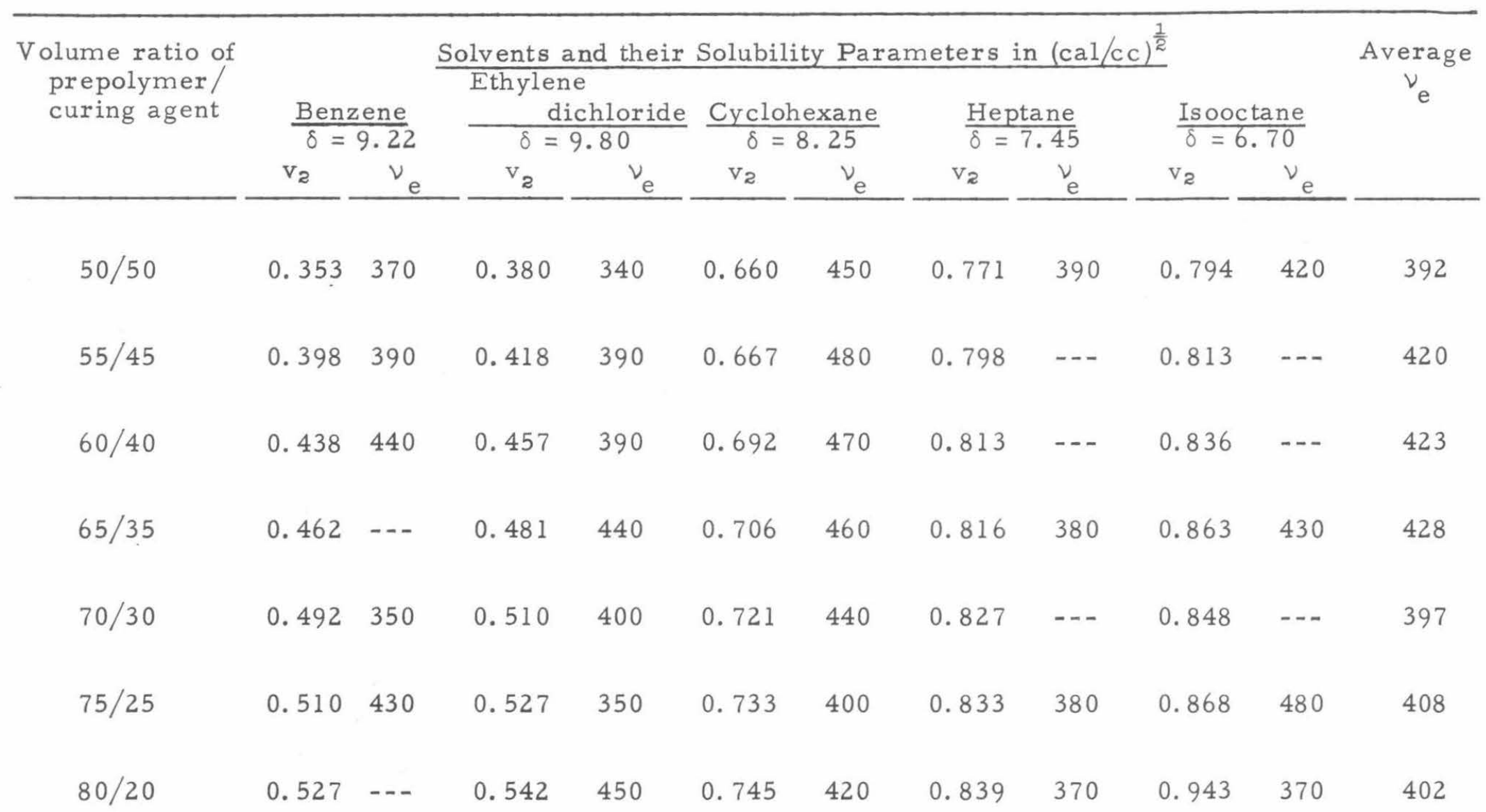




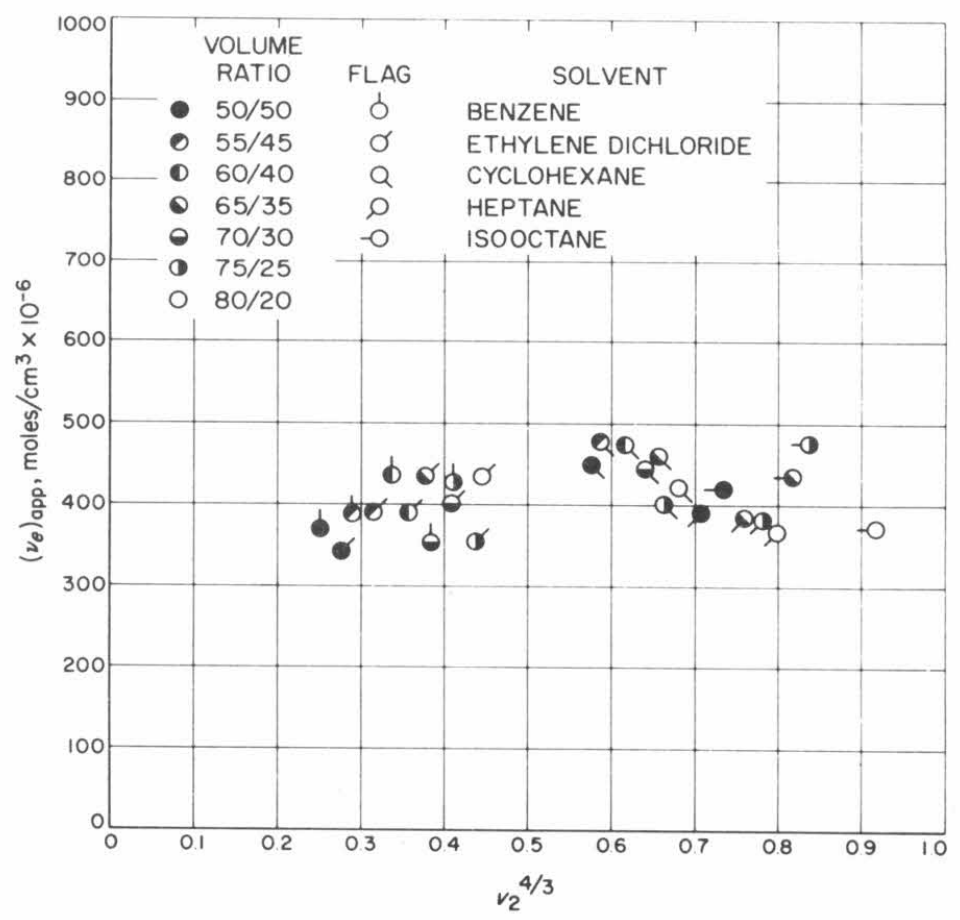

Fig. 2. Dependence of Apparent Chain Concentration, $\left(\nu_{\mathrm{e}}\right)_{\mathrm{app}}$, on $\mathrm{v}_{\mathbf{z}}{ }^{4 / 3}$.

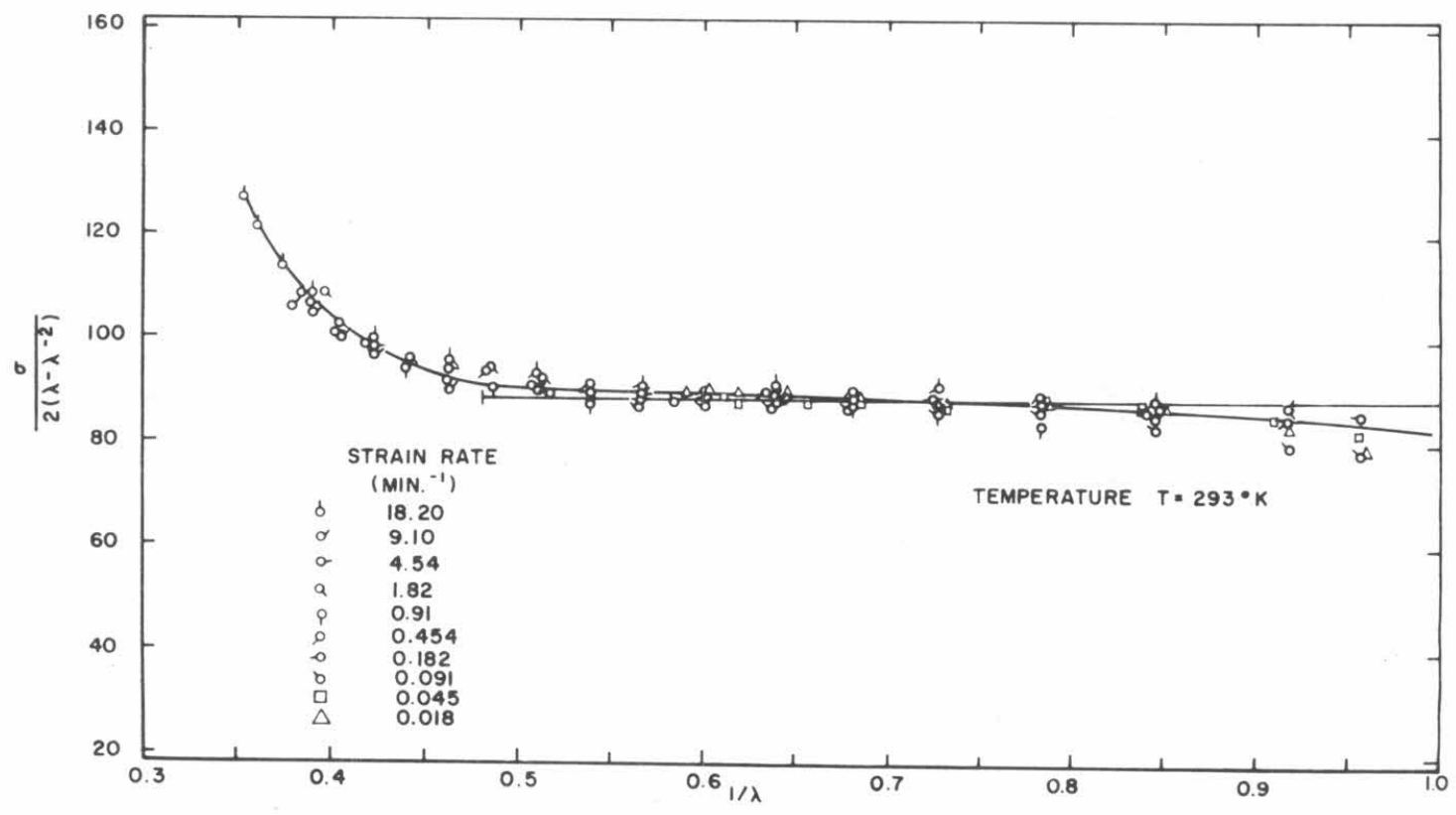

Fig. 3. Mooney-Rivlin Plot for GALCIT I at $20^{\circ} \mathrm{C}$. (From H. K. Mueller, Ref. 5). 


\section{Dependence of the Failure Envelope on Prepolymer Ratio}

It has been shown ${ }^{(2)}$ that the failure envelopes for many gum elastomers can be represented by a stress-strain equation proposed by Treloar ${ }^{(4)}$ :

$$
\sigma_{b}=\frac{\nu e^{R \operatorname{Tn}^{\frac{1}{2}}}}{3}\left(x-\frac{y}{\lambda_{b}{ }^{\frac{3}{2}}}\right)
$$

where

$$
x=\mathcal{L}^{-1}\left(\lambda_{b} / n^{\frac{1}{2}}\right)
$$

and

$$
y=\mathcal{L}^{-1}\left(1 / \lambda_{b}{ }^{\frac{1}{2}} n^{\frac{1}{2}}\right)
$$

Here $\mathcal{L}^{-1}(\xi)$ is the inverse of the Langevin function $\mathcal{L}(\xi)=\operatorname{coth} \xi-1 / \xi$, $\sigma_{b}$ and $\lambda_{b}$ are the stress and extension ratios at break, respectively, and $\mathrm{n}$ is the number of equivalent random links per network chain. By plotting

$$
\log \left(\frac{3 \sigma_{b}}{v_{e^{R T n^{\frac{1}{2}}}}}\right) \text { versus } \log \left(\frac{\lambda_{b}}{n^{\frac{1}{2}}}\right)
$$

for several values of $\mathrm{n}$, a family of failure envelopes is obtained which can then be used to obtain approximate values for both $\nu_{e}$ and $n$ from failure envelope data obtained experimentally. In practice the experimental data, plotted in the form $\log \left(\sigma_{\mathrm{b}} \mathrm{T}_{\mathrm{o}} / \mathrm{T}\right)$ versus $\log \lambda_{\mathrm{b}}$, are shifted horizontally and vertically in order to superpose the experimental data to a theoretical curve. Since the shape of the envelope depends strongly on the $n$ values, this matching or superposition is done on the basis of curve shape 
and as such is readily accomplished. The distance along the abscissa needed for the shift or fit is equal to $\log n^{\frac{1}{2}}$ while the distance along the ordinate is equal to $\log \left(3 / \nu_{e} R T_{0} n^{\frac{1}{2}}\right)$. Thus, estimates for both $n$ and $\nu_{e}$ can be calculated from the shift distances.

Typical plots of the experimentally obtained failure envelope and that predicted on the basis of Eq. (2) are shown in Fig. 4 for the 50/50 and

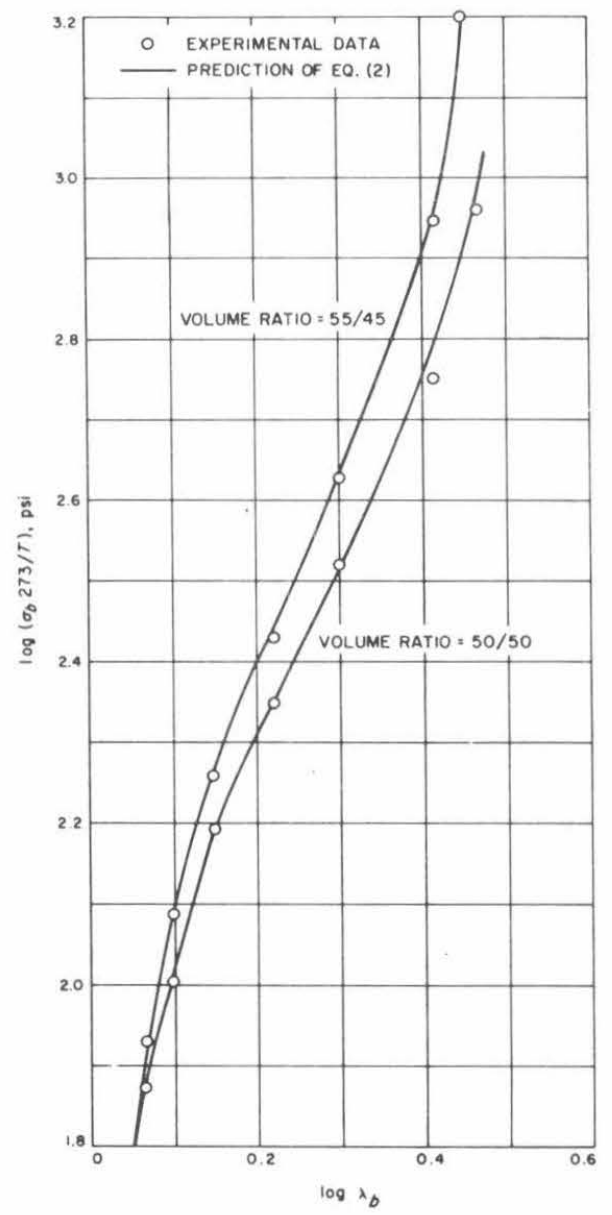

Fig. 4. Failure Envelopes for the 50/50 and 55/45 Solithane Compositions.

and 55/45 ratios. Inspection of the figure makes it evident that the shapes of the experimental failure envelopes and those predicted by Eq. (2) are in 
excellent agreement. Similar results were obtained for the remaining elastomers and the data for $\nu_{e}$ and $n$ evaluated by this procedure are tabulated in Table II.

TABLE II

Estimates of $\nu_{e}$ and $n$ from Failure Envelopes

\begin{tabular}{|c|c|c|}
\hline $\begin{array}{l}\text { Volume ratio of } \\
\text { prepolymer / } \\
\text { curing agent }\end{array}$ & $\begin{array}{c}\nu_{\mathrm{e}}, \mathrm{moles} / \mathrm{cm}^{3} \\
\times 10^{-6}\end{array}$ & $\begin{array}{l}\mathrm{n} \text {, equiv. } \\
\text { random links/chain }\end{array}$ \\
\hline $50 / 50$ & 410 & 12 \\
\hline $55 / 45$ & 460 & 10 \\
\hline $60 / 40$ & 440 & 7.2 \\
\hline $65 / 35$ & 470 & 6.6 \\
\hline $70 / 30$ & 490 & 4.8 \\
\hline $75 / 25$ & 460 & 4. 2 \\
\hline $80 / 20$ & 460 & 4. 2 \\
\hline
\end{tabular}

These results also demonstrate that $\nu_{e}$ is indeed essentially independent of the ratio of prepolymer/curing agent; the small differences observed are well within the estimated error involved in the superposition procedure. Furthermore, it is apparent that the magnitude of $\nu_{\mathrm{e}}$ obtained in this manner is in essential agreement with $\nu_{e}$ determined from the compression measurements on swollen samples: The average value of $\nu_{\mathrm{e}}$ obtained from the failure envelope results is $460 \times 10^{-6}$ compared to 410 $\times 10^{-6}$ moles $/ \mathrm{cm}^{3}$ estimated from the compression results.

If it is assumed that all network chains are effective, then the average value of $\nu_{\mathrm{e}} \approx 440 \times 10^{-6}$ moles $/ \mathrm{cm}^{3}$ can be used to obtain an 
estimate for the maximum value of the average molecular weight of a network chain, $M_{c}$, since for the present castor oil system, chain entanglements are expected to be absent because the molecular weight between chemical crosslinks is so low. Using the relationship $M=\rho / \nu$ where $\rho$ is the polymer density, $\sim 1.00 \mathrm{~g} / \mathrm{cm}^{3}, \mathrm{M}_{\mathrm{c}} \approx 2300 \mathrm{~g} / \mathrm{mole}$. For the real network, $M_{C}$ is expected to have a somewhat smaller value, since not all of the chains in the gel are effective and hence capable of supporting an equilibrium load. In principle, the fraction of "active" network capable of permanent elastic deformation can be estimated if the functionality of the reactants, the extent of reaction, and the initial ratio of active groups are known. The value of the fraction of "active" network will be a maximum when this ratio is unity, if all other parameters are held constant. On the other hand, this fraction will be markedly reduced if the ratio differs appreciably from unity. This will occur in practice if the "purity" of the reactants is low, i. e., if either the prepolymer or the curing agent contains appreciable amounts of monofunctional or difunctional molecules.

A minimum value for $M_{c}$ can be estimated by assuming that all the network chains are effective and, further, that they correspond to the structure obtained from an equimolar reaction of the prepolymer and catalyst. Since the former is tolylene diisocyanate-capped castor oil and the latter is castor oil, for pure materials the $\mathrm{M}_{\mathrm{c}}$ would be $780 \mathrm{~g} / \mathrm{mole}$. The true $M_{c}$ will have a value between the two extremes, i. e. between 780 and $2300 \mathrm{~g} / \mathrm{mole}$.

Based on the manufacturer's specifications for equivalent weight and density, the equivalents of $\mathrm{NCO}+\mathrm{OH}$ per $\mathrm{cm}^{3}$ can be calculated to be 2. $74 \times 10^{-3}$ for all formulations studied. Since, as just noted, the average value of $\nu_{e}$ is $440 \times 10^{-6}$ moles $/ \mathrm{cm}^{3}$, this indicates that there are 
$\frac{1}{2} \times\left(2.74 \times 10^{-3}\right) / 440 \times 10^{-6}$ equivalents incorporated into each chain, or 3. 1 reaction sites per chain between crosslinks rather than the single site expected. This value may also be compared with the value of 2.9 reaction sites per chain obtained from the ratio of the actual to the theoretical $\mathrm{M}_{\mathrm{C}}$ values, $2300 / 780$. Both arguments point to an average functionality which is less than 3 . If there are three reaction sites per chain, this implies that there are 3 difunctional molecules present for every trifunctional one and the chains have a structure such as shown in Fig. 5.

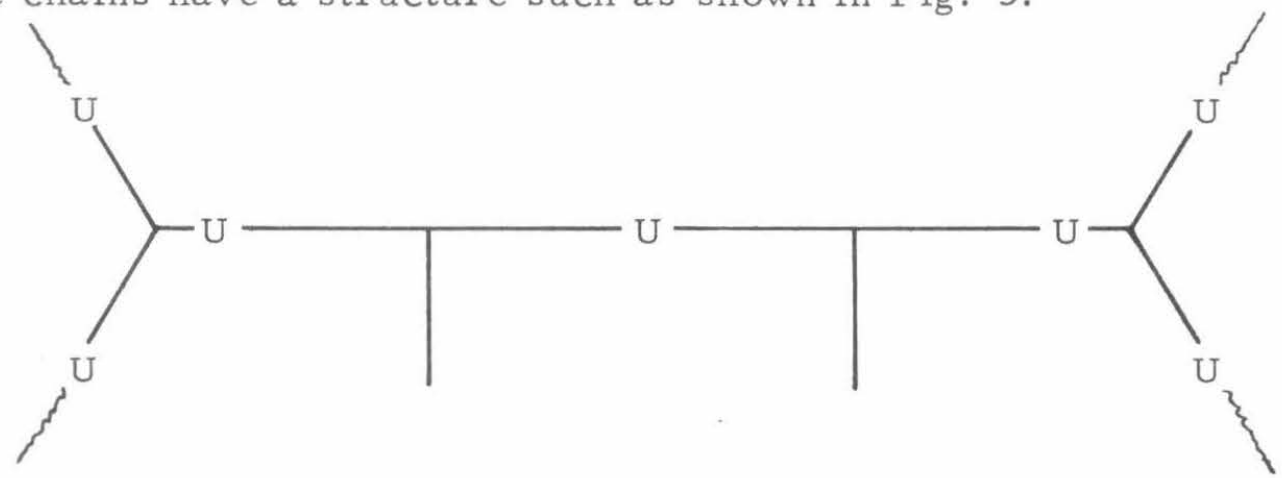

Fig. 5. Sketch of the Chain Structure between Crosslinks for GALCIT I. Each line segment represents a ricinoleic acid moiety; each $U$, the di-urethane as sociated with TDI.

The interesting feature of Table II is the value of $n$, which decreases monotonically with increase in the prepolymer content of the elastomer. A decrease in $\mathrm{n}$ signifies that chain flexibility decreases; i. e., the network chains become more rigid as the prepolymer content increases. Such a change implies a change in the chemical structure of the network chains. However, since the main chains are the same type, castor oil, the only variables would seem to be the chemical nature and functionality of the crosslink. 
It is known that the reaction between an $\mathrm{OH}$ group and an NCO group is relatively rapid. However, the resulting urethane linkage contains an active hydrogen which is capable of reacting, although at a much slower rate, with additional NCO to generate an allophanate ${ }^{(6)}$ according to the scheme shown in Fig. 6. It is important to notice that in this reaction the active hydrogen is regenerated and is thus available for further reaction. For example, the reaction producing the allophanate group might lead to the formation of a network chain; the reaction of a second isocyanate group would lead to a trifunctional branch point; the reaction of a third to a tetrafunctional branch point; etc. The reaction of an isocyanate with a urethane could explain, in part, the fact that $\nu_{e}$ is independent of the prepolymer/ curing agent ratio while the $n$ values decrease with increase in the prepolymer content - this in spite of the fact the $\mathrm{NCO}$ and $\mathrm{OH}$ contents were

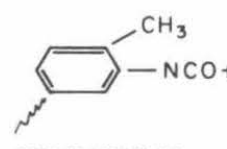

ISOCYANATE

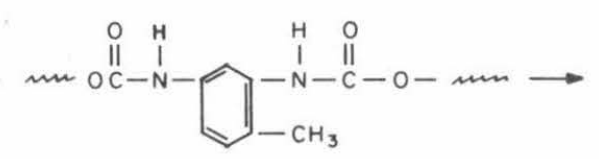

URETHANE

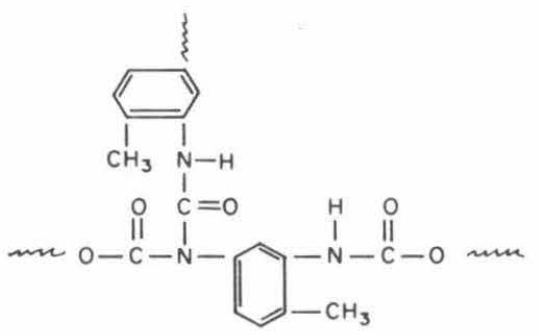

ALLOPHANATE

Fig. 6. Reaction of Isocyanate and Urethane Group to Form Allophanate.

varied as shown in Table I. On the assumption that the reaction of two functional groups increases the number of network chains by one, then $\nu_{\mathrm{e}}$ should be independent of prepolymer content since calculation shows that 
the concentration of reactive groups is independent of prepolymer content. In addition, as the prepolymer content increases, the $\mathrm{OH}$ content decreases and hence both the number of allophanate groups and the extent of branching will increase. Since the allophanate group contains more polar groups than does a urethane group (Fig. 6), the allophanate must confer added rigidity to a network chain, which in turn implies that $n$ will decrease. Thus, on this basis, the decrease in the $\mathrm{n}$ value is a reflection of the increase in the allophanate content.

\section{Interaction Parameters}

According to the usual approximations ${ }^{(7)}$, the relationship between the extent of equilibrium swelling and the chain concentration depends primarily on the molar volume of solvent, $V_{1}$, and the polymer-solvent interaction parameter $\chi_{1}$. The explicit dependence is given by ${ }^{(8)}$ :

$$
\nu_{\mathrm{e}}=-\frac{1}{\mathrm{v}_{1}} \frac{\left\{\ln \left(1-\mathrm{v}_{2}\right)+\mathrm{v}_{2}+\mathrm{v}_{2}^{2}\left[\mathrm{x}_{1}-\left(1-\mathrm{v}_{2}\right) \mathrm{d} \mathrm{x}_{1} / \mathrm{dv_{2 }}\right]\right\}}{\left(\mathrm{v}_{2}^{\frac{1}{3}} \mathrm{~g}^{\frac{2}{3}}-\mathrm{v}_{2} / f\right)}
$$

where $g$ is the gel fraction and $f$ is the crosslink functionality, which is here taken to be 3 . Since $\nu_{\mathrm{e}}$ has been estimated by an independent method, Eq. (5) can be used to evaluate the interaction parameter $X_{1}$. Thus, Eq. (5) can be rearranged to yield:

$$
\frac{\nu_{\mathrm{e}} \mathrm{v}_{1} \mathrm{v}_{2}^{\frac{1}{3}} \mathrm{~g}^{\frac{2}{3}}-\left(\mathrm{v}_{2} / 3\right)+\ln \left(1-\mathrm{v}_{2}\right)+\mathrm{v}_{2}}{\mathrm{v}_{2}^{2}}=-\left[\mathrm{x}_{1}-\left(1-\mathrm{v}_{2}\right) \mathrm{d} \mathrm{x}_{1} / \mathrm{dv_{2 }}\right]
$$


Furthermore, the dependence of $\chi_{1}$ on $v_{z}$ is generally of the form:

$$
x_{1}=a+b v_{2}
$$

where $\mathrm{a}$ and $\mathrm{b}$ are constants. Assuming Eq. (7) to be valid, the righthand side of Eq. (6) becomes $(a-b)+2 b_{2}$; thus a plot of the left-hand side of the equation $\left[\equiv\left(x_{1}\right)_{\text {app }}\right]$ versus $v_{2}$ should be linear with slope $2 b$ and intercept $(a-b)$. These results are shown in Fig. 7 , where it may be seen that the plots are indeed linear for all the solvents investigated. This in turn justifies the assumed linear dependence of $X_{1}$ on $v_{2}$, i.e., Eq. (7). From the slope and intercept, values of $\mathrm{a}$ and $\mathrm{b}$ were obtained and the se are listed in Table III. These $X_{1}$ values may be used along with Eq. (5) to estimate $\nu_{e}$ values from measurements of $v_{z}$.

TABLE III

Polymer-solvent Interaction Parameters

\begin{tabular}{lccc}
\hline \multicolumn{1}{c}{ Solvent } & $\mathrm{v}_{1}$ & $\begin{array}{c}\delta_{\mathrm{s}} \\
\left(\mathrm{cal} / \mathrm{cm}^{3}\right)^{\frac{1}{2}}\end{array}$ & $\chi_{1}$ \\
\hline $\begin{array}{l}\text { Benzene } \\
\begin{array}{l}\text { Ethylene } \\
\text { dichloride }\end{array}\end{array}$ & 88.9 & 9.22 & $0.69+0.63 \mathrm{v}_{\mathbf{z}}$ \\
$\begin{array}{l}\text { Cyclohexane } \\
\text { Heptane }\end{array}$ & 108 & 9.80 & $0.70+0.60 \mathrm{v}_{\mathbf{z}}$ \\
Isooctane & 146.5 & 7.45 & $0.58+0.99 \mathrm{v}_{\mathbf{z}}$ \\
& 165.1 & 6.90 & $0.16+1.8 \mathrm{v}_{\mathbf{z}}$ \\
\hline
\end{tabular}



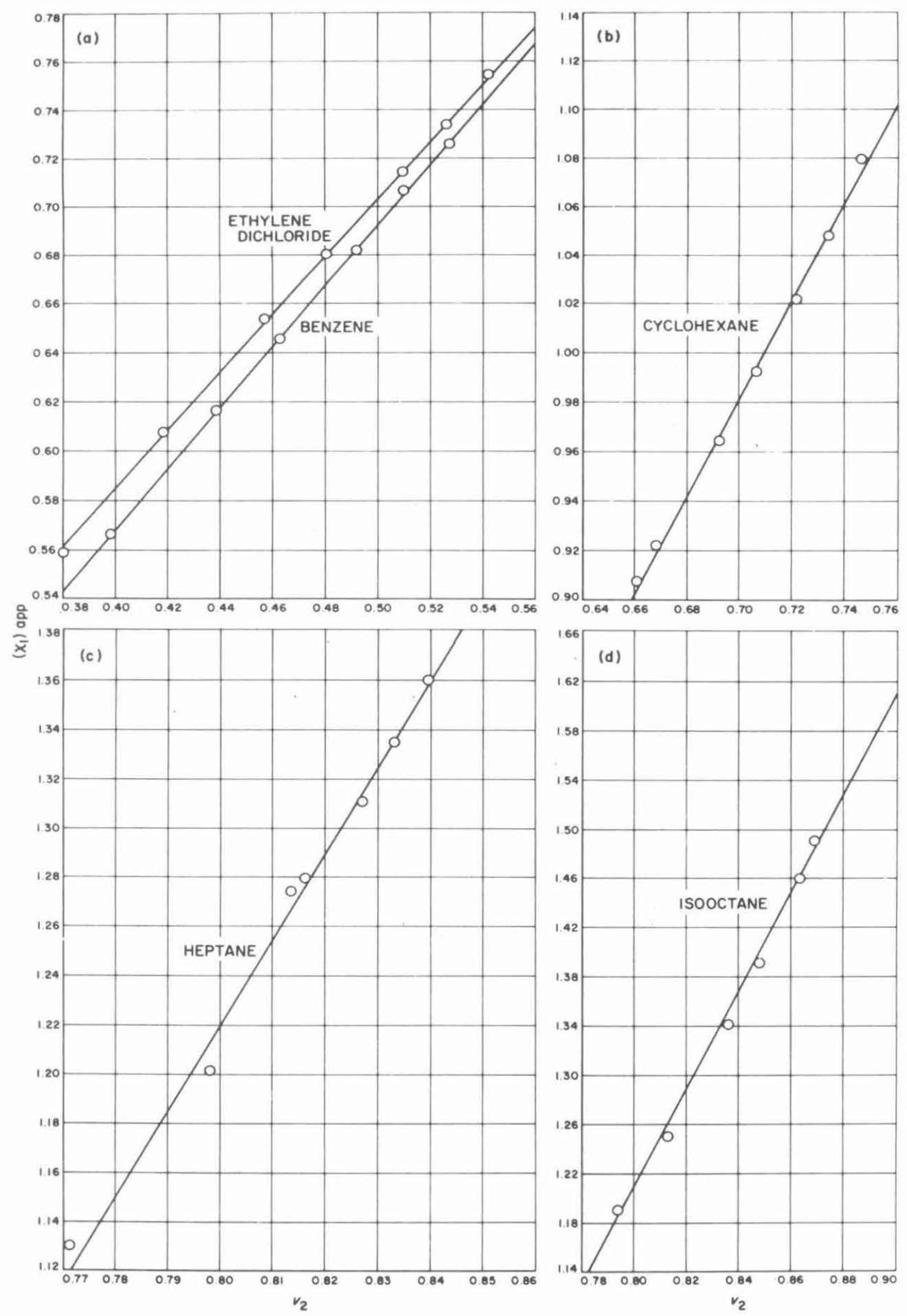

Fig. 7. Dependence of Apparent Polymer-Solvent Interaction Parameter, $\left(x_{1}\right)_{a p p}$, on $v_{2}$. 
If it is further assumed that the variations in the chemical structure of the network chains do not influence $\chi_{1}$ in the limit when $v_{2}$ approaches unity (i.e., for the undiluted polymers), then the $\chi_{1}$ values listed in Table III may be used to obtain a rough estimate for the cohesive energy density, $\delta_{p}$, of the polymer. It has been shown that $\chi_{1}$ is related to the cohesive energy density of the solvent and the polymer by means of (9)

$$
x_{1}=A+V_{1} / R T\left(\delta_{s}-\delta_{p}\right)^{2}
$$

where $\mathrm{A}$ is a constant for a given solvent and the subscripts $\underline{s}$ and $\underline{p}$ refer to the solvent and the polymer, respectively. Since A is presumed independent of $\mathrm{v}_{\mathfrak{z}}$, differentiation of Eq. (8) with respect to $\mathrm{v}_{\mathbf{z}}$ yields

$$
\frac{d x_{1}}{d v_{2}}=-\frac{2 v_{1}}{R T}\left(\delta_{s}-\delta_{p}\right) \frac{d \delta_{p}}{d v_{2}}
$$

Further, in the limit as $v_{2}$ approaches unity, $d \delta_{p} / d v_{z}$ should be, to a good approximation, independent of the solvent. Thus, Eq. (9) can be written as

$$
\frac{R T b}{2 V_{1}}=-B \delta_{s}+\delta_{p} B
$$

where $\mathrm{B} \equiv \mathrm{d} \delta_{\mathrm{p}} / \mathrm{dv}_{2}, \mathrm{~b} \equiv \mathrm{d} x_{1} / \mathrm{dv}_{2}$. A plot of $\mathrm{RTb} / 2 \mathrm{~V}_{1}$ versus $\delta_{\mathrm{S}}$ is predicted to be linear with slope of $-\mathrm{B}$ and intercept $\mathrm{B} \delta_{\mathrm{p}}$. Figure 8 shows the data plotted in this manner using the $\delta_{\mathrm{S}}$ values listed in Table III. Although the scatter is relatively large, the data do seem to conform to Eq. (10), and $\delta_{p}$ is calculated from the slope and intercept to be $11.6\left(\mathrm{cal} / \mathrm{cm}^{3}\right)^{\frac{1}{2}}$. 


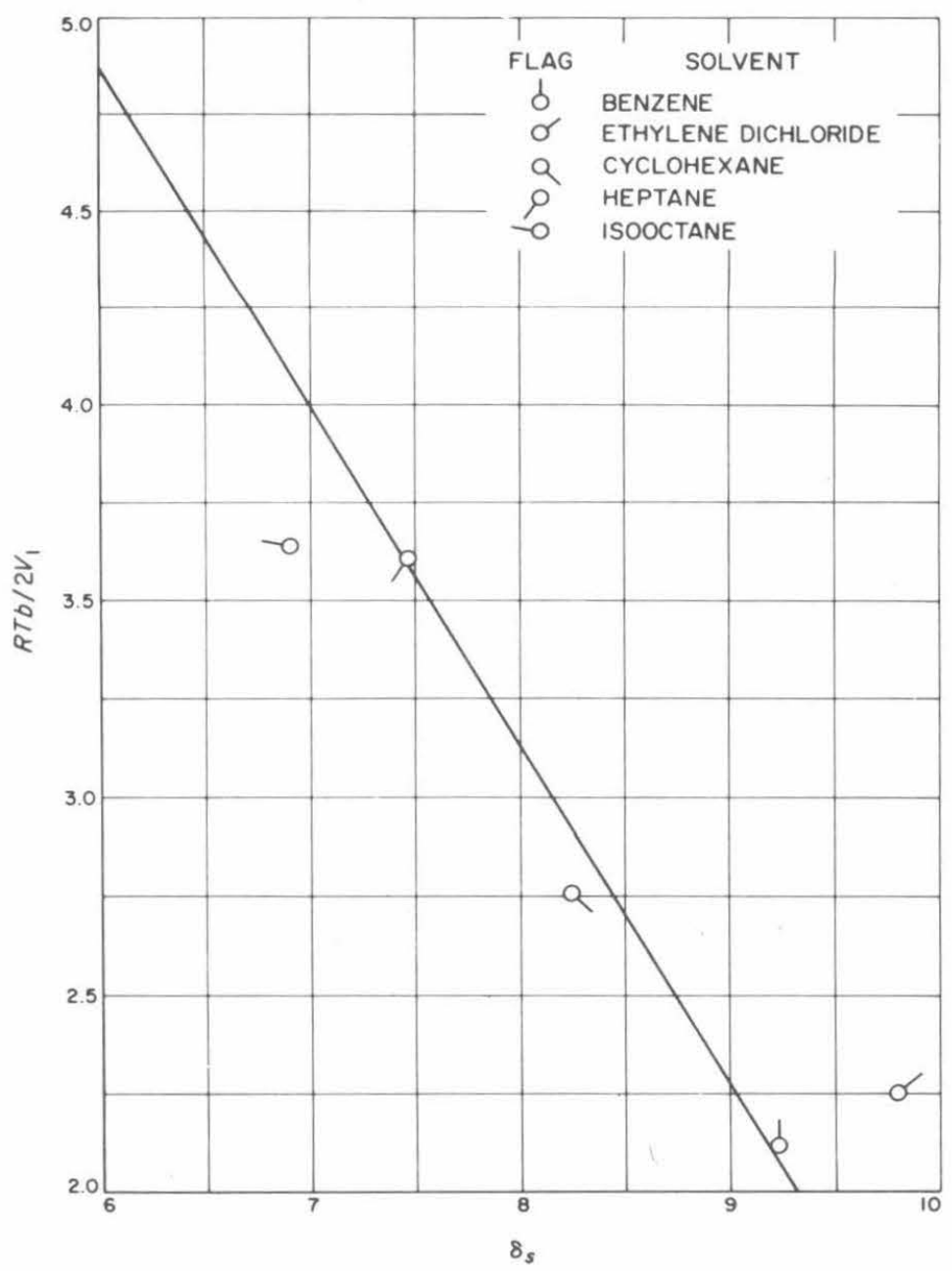

Fig. 8. Dependence of $R \mathrm{~Tb} / 2 \mathrm{~V}_{1}$ on $\delta_{\mathrm{s}}$. 


\section{REFERENCES}

1. Knauss, W. G., Clauser, J. F., and Landel, R. F., AFRPL-TR66-21, Edwards, California, January 1966, MATSCIT PS 66-1, California Institute of Technology, Pasadena, California, January 1966.

2. Landel, R. F., and Fedors, R. F., Rubber Chemistry and Technology, (in press).

3. Fedors, R. F., and Landel, R. F., Jet Propulsion Laboratory Space Programs Summary 37-43, Vol. IV, Pasadena, California, February 28, 1968, p. 177.

4. Treloar, L. R. G., The Physics of Rubber Elasticity, Oxford University Press, (1958).

5. Mueller, H. K., Ph. D. Thesis, California Institute of Technology, Pasadena, California (1968).

6. Saunders, J. H., Rubber Chemistry and Technology, Vol. 32, p. 337, (1959).

7. Flory, P. J., Principles of Polymer Chemistry, Cornell University Press, (1953).

8. Holly, E. D., J. Polym. Sci., Vol. A2, p. 5267, (1964).

9. Huggins, M. L., Physical Chemistry of High Polymers, John Wiley and Sons, Inc., New York, (1958).

10. Sheehan, C. J., and Bisio, A. L., Rubber Chemistry and Technology, Vol. 39, p. 149, (1966). 

PART V

FINITE DEFORMATION STUDIES

R. G. Mancke

and

R. F. Landel 
PART V

FINITE DEFORMATION STUDIES

\section{CONTENTS}

1. Introduction . . . . . . . . . . . . . . . . V - 1

2. Theory . . . . . . . . . . . . . . . . . . . . . $\quad$. 4

3. Experimental . . . . . . . . . . . . . . . V -6

4. Results and Discussion. . . . . . . . . . . . $\mathrm{V}-10$

References . . . . . . . . . . . . . . . . $\quad \mathrm{V}-18$ 


\section{ILLUSTRATIONS}

Fig. 1a Invariant $I_{1}$ Plotted against Invariant $I_{2}$ for Specimens with Undeformed Length $2 \mathrm{~cm}$ and Undeformed Diameter $1 \mathrm{~cm}$, using Eqs. (2). . . . V - 3

Fig. 1b As Fig. la for $2 \times 2 \mathrm{~cm}$ Specimens . . . . . . V V - 3

Fig. 2 Moment vs. Torsion and Tension, Natural Rubber, $1 \% \mathrm{~S}$. . . . . . . . . . . V V 11

Fig. 3 True Stress vs. Torsion and Tension, Natural Rubber, $1 \% \mathrm{~S}, 2 \times 2$ Specimens. . . . . V -13

Fig. 4 True Stress vs. Torsion and Tension, Natural Rubber, $1 \%$ S . . . . . . . . . . V -14 
TABLES

Table I Analysis of the Accumulated Natural Rubber 1\% Sulfur Data Using Equations (5) and (6);

All Values are to be Considered Approximate and Temporary, as Discussed in the Text . . . . V V 15 


\section{Introduction}

According to the theory of finite deformation of continua, the stress-strain behavior of a material under any type of deformation can be simply and concisely expressed in terms of the elastically stored free

energy, $w^{(1)}$. This quantity is a function of the three invariants of the strain tensor, $I_{i}$, and also a function of the time $t$ and the loading history if the material is viscoelastic. In order to determine the form of $W$, it is necessary to carry out experiments in which the strains, and hence the stresses, are different in each of the three principal directions. For a viscoelastic material, the problem of the time-dependence can be simplified by first determining $W$ for isochronal data.

Previous work on time-dependent and isochronal stored energy functions for elastomers has led to many.proposed equations for W based on the use of the strain invariants $(2-18)$, and one equation based on the use of the stretch ratios as the measure of the strain (19). (The prior work referred to here is limited to those proposals that have been experimentally tested.) Some of these equations for W are equivalent; for example, the Bernstein-Kearsley-Zapas $(\mathrm{BKZ})^{(5)}$ and Lianis ${ }^{(7)}$ equations are formally equivalent to the Signorini form ${ }^{(13)}$ of the dependence of $W$ on the invariants for incompressible materials:

$$
W=C_{20}\left(I_{1}-3\right)+C_{01}\left(I_{2}-3\right)+C_{20}\left(I_{1}-3\right)^{2}
$$

where the coefficients $C_{i j}$ are material functions of time. Some of the proposed forms fit uniaxial extension data quite well but can not then fit data in uniaxial compression; the simple Mooney expression widely used in the literature (Eq. (1) with $\mathrm{C}_{20}=0$ ) is an example. In fact it has been 
argued that no simple power series expression with only a few terms can give a valid description because the gradients $\partial W / \partial I_{i}$ are sharply curved in the region of small strains $(10,20,21)$.

One type of simple biaxial experiment is achieved by the BeckerLandel torsion-tension tester, ${ }^{(22)}$ which simultaneously twists and either extends or compresses a solid cylindrical sample and records the moment and axial force as functions of time. These data can be used to test proposed forms of $\mathrm{W}$, or, with more difficulty, W can be determined directly. The capability of measuring in compression as well as extension is a great asset, as can be seen in Figs. la and 1b. Figure la shows the values of $I_{1}$ and $I_{2}$ that are accessible with a sample $2 \mathrm{~cm}$ long and $1 \mathrm{~cm}$ in diameter for strains from about $60 \%$ compression to $200 \%$ extension and twists of $0^{\circ}$ to $360^{\circ}$. Figure $1 \mathrm{~b}$ gives similar information for a $2 \times 2 \mathrm{~cm}$ rod. The invariants are given (for an incompressible material*) by (23)

$$
\begin{aligned}
& I_{1}=\lambda^{2}+2 \lambda \lambda+r^{2} \psi^{2} \lambda^{2} \\
& I_{2}=2 \lambda+1 / \lambda^{2}+r^{2} \psi^{2} \lambda
\end{aligned}
$$

where $\lambda$ is the ratio of deformed and undeformed lengths, $\psi$ is the twist in radians per unit deformed length, and $r$ is the radial distance from the axis to the material point in the deformed sample. The line $I_{1}=I_{2}$ corresponds to twist without extension (pure shear), and all data lying above it refer to extension; below it, to compression. It is apparent that new

* The slight volume change induced by the extension is not detectable by normal means of direct strain measurement, though it is readily observed in more sensitive density change measurements (Ref. 24). Hence we consider the material to be incompressible in subsequent discussion here. 


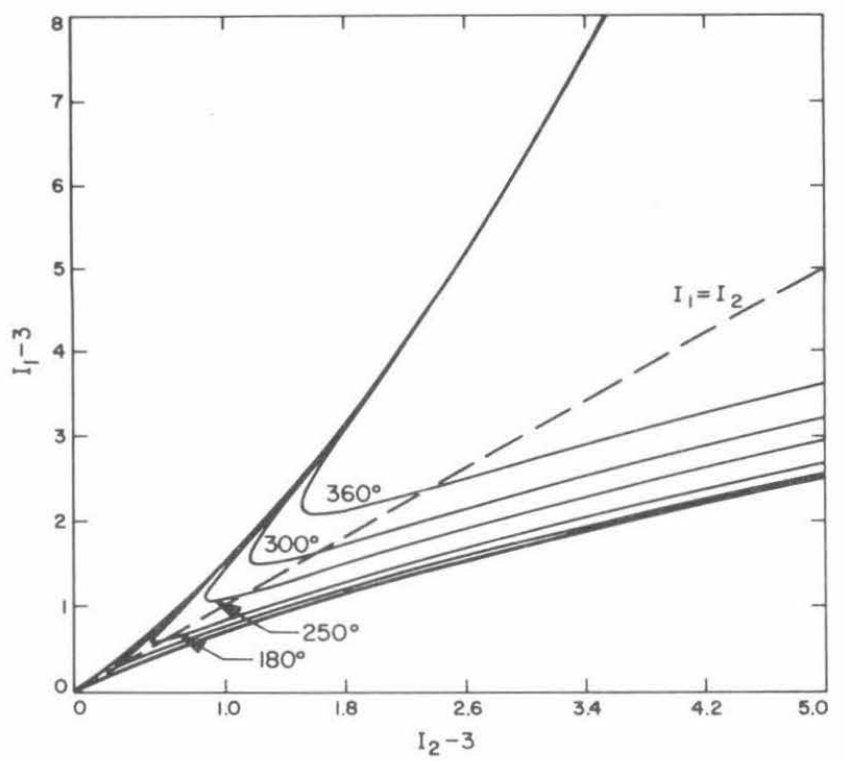

Fig. la. Invariant $I_{1}$ Plotted against Invariant $I_{2}$ for Specimens with Undeformed Length $2 \mathrm{~cm}$ and Undeformed Diameter $1 \mathrm{~cm}$, using Eqs. (2).

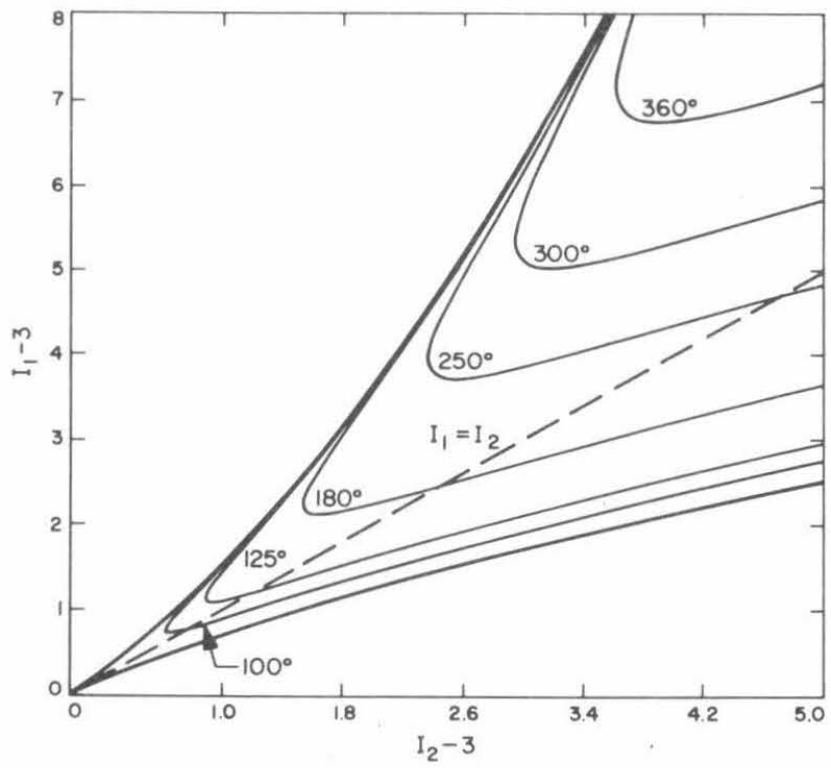

Fig. lb. As Fig. la for $2 \times 2 \mathrm{~cm}$ Specimens. 
regions of the $I_{1}-I_{2}$ plane become theoretically accessible in compression. In practice, however, the ranges in compression are limited by buckling of the specimen.

The theory of the torsion-tension experiment, as expressed in terms of the invariants, is due to Rivlin (23). For incompressible materials, the results lead to the following expressions for the force (not stress), $N$, on the end of the rod, and the moment, $M$ :

$N=4 \pi\left(\lambda-\lambda^{-2}\right) \int_{0}^{d / 2}\left(\lambda W_{1}+W_{2}\right) r d r-2 \pi \lambda \psi^{2} \int_{0}^{d / 2}\left(\lambda W_{1}+2 W_{2}\right) r^{3} d r$

$M=4 \pi \psi \lambda \int_{0}^{d / 2}\left(\lambda W_{1}+W_{z}\right) r^{3} d r$

where $d$ is the deformed diameter, $W_{1}$ is $\left(\partial W / \partial I_{1}\right)_{I_{2}, t}$ and $W_{2}$ is $\left(\partial \mathrm{W} / \partial \mathrm{I}_{2}\right)_{I_{1}, t}$. In this report we use Eqs. (2) - (4) to determine $\mathrm{N}$ and $M$ as functions of $\lambda$ and $\psi$ for the Signorini form of $W$, and we give the relationships between the material functions of Eq. (1) and those of the Lianis - BKZ material. We then test these equations with an extensive series of data on natural rubber cross-linked with $1 \%$ sulfur $(22,25)$. Direct evaluation of $W_{1}$ and $W_{2}$ is reserved for a later report.

\section{Theory}

Using Eqs. (1) - (4) and carrying out the necessary computations, the following expressions are obtained for $\mathrm{N}$ and $\mathrm{M}$ : 
$N=\frac{\pi d^{2}}{4}\left(\lambda-\lambda^{-2}\right)\left[\left(2 C_{01}+8 C_{20}\right)+\left(2 C_{10}-8 C_{20}\right) \lambda+4 C_{20}\left(\lambda^{3}-\lambda+\frac{d^{2} \lambda^{3} \psi^{2}}{8}\right)\right]$

$-\frac{\pi d^{4} \lambda \psi^{2}}{64}\left[2\left(2 C_{01}+8 C_{20}\right)+\left(2 C_{10}-8 C_{20}\right) \lambda+4 C_{20}\left(\lambda^{3}-\lambda-2+\frac{d^{2} \lambda^{3} \psi^{2}}{6}\right)\right]$

$M=\frac{\pi d^{4} \lambda \psi}{32}\left[\left(2 C_{01}+8 C_{20}\right)+\left(2 C_{10}-8 C_{20}\right) \lambda+4 C_{20}\left(\lambda^{3}-\lambda+\frac{d^{2} \lambda^{3} \psi^{2}}{6}\right)\right.$

Except that our $d / 2$ is equal to their $d$, these equations become identical to the Goldberg-Lianis ${ }^{(26)}$ Eqs. (18) and (19) when the following substitutions are made:

$$
\begin{aligned}
& \alpha(t)=4 C_{20}(t), \\
& \beta(t)=2 C_{0_{1}}(t)+8 C_{20}(t), \text { and } \\
& Y(t)=2 C_{10}(t)-8 C_{20}(t) .
\end{aligned}
$$

However, for the remainder of this report we will refer to isochronal data only, and we therefore shorten the notation to $\alpha, \beta, \gamma, \mathrm{C}_{0_{1}}, \mathrm{C}_{10}$, and $\mathrm{C}_{20}$.

Equation (6) suggests that the moment should be directly proportional to $\psi$ if $C_{20}=0$ (Mooney form); otherwise $M / \psi$ should be proportional to $\psi^{2}$. Hence, $M$ offers a simple means of testing the adequacy of Eq. (1). In the absence of torsion, Eq. (5) can be tested in the usual fashion by plotting $\sigma /\left(\lambda-\lambda^{-2}\right)$ versus $1 / \lambda$, where $\sigma$ is $N / A_{0}, A_{0}$ being the initial cross-sectional area. Recognizing that $\pi d^{2} / 4$ is $A_{0} \lambda^{-1}$, then if $C_{20}=0$, a straight line will be obtained, with slope $2 \mathrm{C}_{0_{1}}$ and intercept $2 \mathrm{C}_{10}$. We know that the Mooney form is a poor representation of experimental results when uniaxial extension and compression data are both considered. The data of Treloar (27), Rivlin and Saunders ${ }^{(28)}$, Forster ${ }^{(29)}$, and our own data show that the slope of such a plot changes sharply from a large positive 
value in the extension region to much smaller values in the region of compression. Furthermore, Eq. (1) can not explain this change in slope even when $\mathrm{C}_{z \circ}$ is not zero. According to Eq. (5), when $\psi=0$, the slope of such a plot is

$$
\frac{d\left[\sigma /\left(\lambda-\lambda^{-2}\right)\right]}{d(1 / \lambda)}=\beta-\frac{2 \alpha}{(1 / \lambda)^{3}}
$$

which changes smoothly with $1 / \lambda$, in contrast to the observed large change near $\lambda=1$ and nowhere else.

Therefore we know that the Signorini - BKZ - Lianis form of W is doomed to failure. Nevertheless, it is instructive and useful to examine the extent to which the equation can be applied. The engineering profession may find that two separate expressions for W - one for extension and one for compression - may satisfy their needs for a phenomenological representation which is based on and couched in a continuum mechanics framework.

\section{Experimental}

The specimens were molded from natural rubber, using $1 \%$ sulfur, and the same recipe employed for the sheet specimens tested in another Becker-Landel apparatus $(30,31)$. Three specimen sizes were used, $2 \mathrm{~cm}$ long $\times 1 \mathrm{~cm}$ diameter, $2 \times 2 \mathrm{~cm}$, and $10 \times 3 \mathrm{~cm}$. A few of the $2 \times 1 \mathrm{~cm}$ and all of the $10 \times 3 \mathrm{~cm}$ specimens were molded from the same batch of rubber. The remainder of the $2 \times 1$ 's and the $2 \times 2$ 's were molded from different batches. All specimens were end-bonded to aluminum tabs with epoxy cement using the technique reported previously $(22)$. 
The biaxial stress relaxometer has been described previously ${ }^{(22)}$. Rod-like specimens are simultaneously twisted and extended or compressed to within a fraction of a percent of the desired strain in less than 0.5 seconds. However, to obtain more precise strains the crosshead must be manually positioned for the final increments, a step that usually takes about ten seconds. The strains were held fixed for 5 - 30 minutes while the axial load and torque were recorded as functions of time. In this report, we will treat only isochronal data taken at five minutes after the start of the experiment. For each test run, either $\lambda$ was held constant and the rotation $\theta$ was increased in steps, or vice versa. After each step, the load was removed for 10 - 15 minutes. Our material is highly cross-linked, and there is very little relaxation after 10 minutes ${ }^{(22)}$; this fact, combined with the stepwise increase in loading, lead us to expect that the measurements will not be affected by the previous steps. This expectation will be considered in the next section.

The rotation $\theta$ was monitored by reading a scale inscribed on a circular plate attached to the crosshead. This plate also has a horizontal reference mark, used to measure the vertical displacement. Originally, the stretch ratios $\lambda$ at the center of the sample were to have been computed from the axial displacements by determining separately an effective gage length, which was expected to be constant for each of the three specimen sizes. However, it was then realized that the fast equilibration of our material to the imposed load allowed an accurate measure of the strained diameter, d, with the cathetometer used to measure the unstrained diameter, $d_{0},($ to $\pm 0.0002 \mathrm{~cm})$, and it is easily shown that $\lambda$ is given exactly, for a perfectly cylindrical portion of an incompressible specimen by 


$$
\lambda=\frac{\mathrm{d}_{0}^{2}}{\mathrm{~d}^{2}}
$$

All values of $\lambda$ used in this report were calculated from Eq. (8).

We can now determine whether the effective gage length, $l_{\text {eff }}$, is constant for each specimen size; $\ell_{\text {eff }}$ is defined by

$$
\lambda=1+\frac{\Delta l}{l_{\text {eff }}}
$$

where $\Delta l$ is the crosshead displacement. A measure of the end effect for $\lambda$ is $l_{0} / l_{\text {eff }}, l_{0}$ being the undeformed sample length. This ratio was found to be dependent on the length-to-diameter ratio, $l_{0} / d_{0}$, of the specimens, as expected, and it was also dependent on $\lambda$, the details of the end-bonding process, and imperfections in the specimen. It varied from about 1.3 to 1.0 for the $2 \times 2 \mathrm{~cm}$ specimens, 1.2 to 1.0 for the $2 \times 1 \mathrm{~cm}$ specimens, and 1.05 to 0.8 for the $10 \times 3 \mathrm{~cm}$ specimens.

The twist variable $\psi$ can not be determined as easily as $\lambda$, so we presumed that the same effective gage length applied for twist as for extension and we approximated $\psi$ as

$$
\psi_{\mathrm{d}}=\frac{\pi \theta / 180}{l_{\text {eff }}+\Delta l}=\frac{\pi \theta}{180 \mathrm{~d}_{\mathrm{o}}{ }^{2}}\left(\frac{\mathrm{d}_{\mathrm{o}}{ }^{2}-\mathrm{d}^{2}}{\Delta l}\right)
$$

where the subscript $\underline{\mathrm{d}}$ refers to diameter. The analysis of the results presented below was based on the $\psi_{d}$ values. Recently we have begun to check this approximation by putting grids on specimens and measuring $\psi$ directly. The measurements have been completed only on one $10 \times 3 \mathrm{~cm}$ specimen, for which $\psi$ is within $1 \%$ of 


$$
\psi_{x h}=\frac{\pi \theta / 180}{l_{0}+\Delta l}
$$

for $\lambda$ from 1.0 to 1.3 and $\theta$ from $50^{\circ}$ to $300^{\circ}$, which are almost the entire ranges of $\lambda$ and $\theta$ that a $10 \times 3 \mathrm{~cm}$ sample would be subjected to. The subscript $\underline{x h}$ refers to crosshead. In other words, for this one specimen there is virtually no end effect for the twist. A final analysis of the data must await completion of these measurements. The grids on the samples were applied by mounting the specimens in a milling machine and a ball-point pen in the tool holder. Also, the smaller mold cavities have been precision engraved with 5 mil grooves spaced $0.250 \pm 0.001$ inches apart along the axis and $15.00 \pm 0.05^{\circ}$ apart around the circumference, and future samples will be molded in both the engraved and smooth configurations. Specimens of both SBR and GALCIT I have been prepared in these grooved molds and no difficulty has been encountered in removing them from the mold cavities.

One instrumentation problem arose during the course of these measurements - - the chart readings at zero loads for the tension and torsion load cells changed as much as $6 \mathrm{~g}$ and $25 \mathrm{~g}$, respectively, during a test run. Separate experiments showed that these zero readings changed with an approximately constant rate, and the data were corrected accordingly. Nevertheless, this problem sometimes leads to noticeable errors, especially in the torque measurements of the smallest specimens, since the forces on the torsion load cell were always less than $400 \mathrm{~g}$ for those specimens. Whatever the cause(s) of these zero reading shifts, the effects on the data are apparent, and incorrect conclusions can be avoided.

We have recently attempted to further understand and control these changes in zero readings. It has always been realized that small changes 
in temperature have noticeable effects ${ }^{(22)}$, and indeed we found the torsion record to drift - $13 \mathrm{~g}$ per ${ }^{\circ} \mathrm{C}$, and the tension record about $-9 \mathrm{~g}$ per ${ }^{\circ} \mathrm{C}$. However we also found that certain conditions produced permanent zero shifts. For example, repeated loading and unloading, and alternation of tension and compression loads, both shifted the tension zero reading. (An identical effect of torsion and reversed torsion is also present in that system, but we believe it to be unimportant because torques are applied in only one direction during measurements). Moreover, the shock on the system from the compressed air piston used to move the crosshead shifted the zeroes of both recorders slightly. Each of these permanent zero shifts may have been caused by small irreversible changes in the system. During this same period the cross-talks between the two load cells (i. e., deflections on one load cell when a load is applied to the other load cell) also changed unaccountably, and this almost certainly indicates an irreversible change somewhere in the system. At about this time the Bendix free-flex pivot between the tension cell and spindle was carelessly broken. With the installation of a new pivot, the tension zero no longer shifted when tensive and compressive loads were alternated. Perhaps these other anomalous zero shifts may also have disappeared; we shall determine whether this is true as soon as possible. If the zero changes now depend only on temperature, they can be calibrated, or the instrument can be placed in a constant temperature environment.

\section{Results and Discussion}

Figure 2 shows most of the moment data, plotted as $32 \mathrm{M} / \pi \mathrm{d}^{4}$ against $\psi_{\mathrm{d}}$. (A pond is a gram force, and 1 pond $/ \mathrm{mm}^{2}=1.422 \mathrm{psi}$ ). 


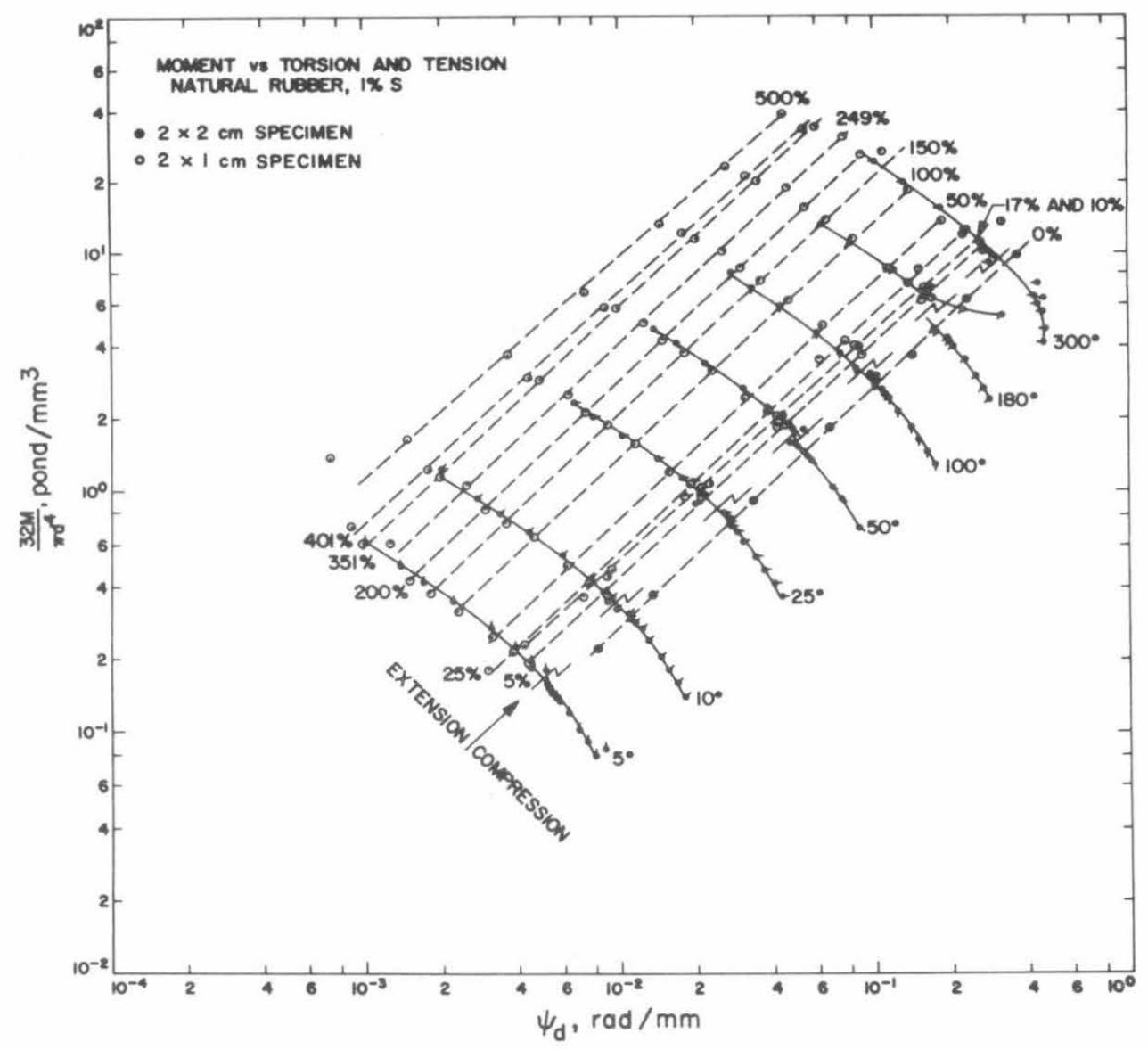

Fig. 2. Moment vs. Torsion and Tension, Natural Rubber, $1 \%$ S.

The solid lines are drawn through the test runs taken at constant rotation, indicated in degrees, and the dashed lines are for test runs at constant strain, indicated in percent crosshead strain, or $100 \mathrm{\Delta l} / \ell_{0}$. (The fact that $\psi_{\mathrm{d}}$ is dependent on strain at constant $\theta$ can be seen from the first part of Eq. (10), since $l_{\text {eff }}$ is approximately constant.) The two types of data agree reasonably well, as shown by the closeness of the solid lines to the open circles, and this agreement lends some support to our expectation that the measurements are not affected by the previous steps of the test runs. The plot also shows the data from the two sample sizes to be 
comparable. The deviations from the pattern at large $\theta$ are not to be trusted, and the smaller deviation of the $500 \%$ strain curve is probably due to crystallization in the rubber. The slopes of the dashed lines are approximately unity, as predicted by Eq. (6) when $\alpha$ is small.

Most of the tensile data are displayed in similar fashion on Figs. 3 and 4. The absolute value of $\sigma$ is plotted in Fig. 3 in order to show the compression data also. The dashed lines have unit slope. No attempt is made to interweave the data taken at constant strains and increasing $\theta$. The figures show that $\sigma$ decreases with increasing twist at constant strain; it becomes negative at large enough twists, though the negative values are not shown on the plots. Also, the curves for all $\theta$ merge together as the strain increases. Both these facts are in general agreement with Eq. (5).

All of the moment data and a small part of the axial force data were compared in detail with Eqs. (5) and (6) by making various plots which are suggested by the forms of those equations. The equations give satisfactory fits of the data, and the best values of $\alpha, \beta$, and $\gamma$ are presented in Table I. As indicated earlier, these results are based on the use of $\Psi$, whereas recent measurements indicate that $\psi_{\mathrm{xh}}$ is a better measure of $\psi$. Hence the values in the table must be considered approximate; we will correct the analysis and present the final results in the near future.

Nevertheless, two general trends to be seen in this table merit comment. First $\gamma$ and $\beta$ values from axial force are, in seven of eight cases, from $12 \%$ to $70 \%$ larger than values from the corresponding moment data. This discrepancy may disappear when the $\psi$ values are corrected. The second, and at the moment more important, trend is that results from 


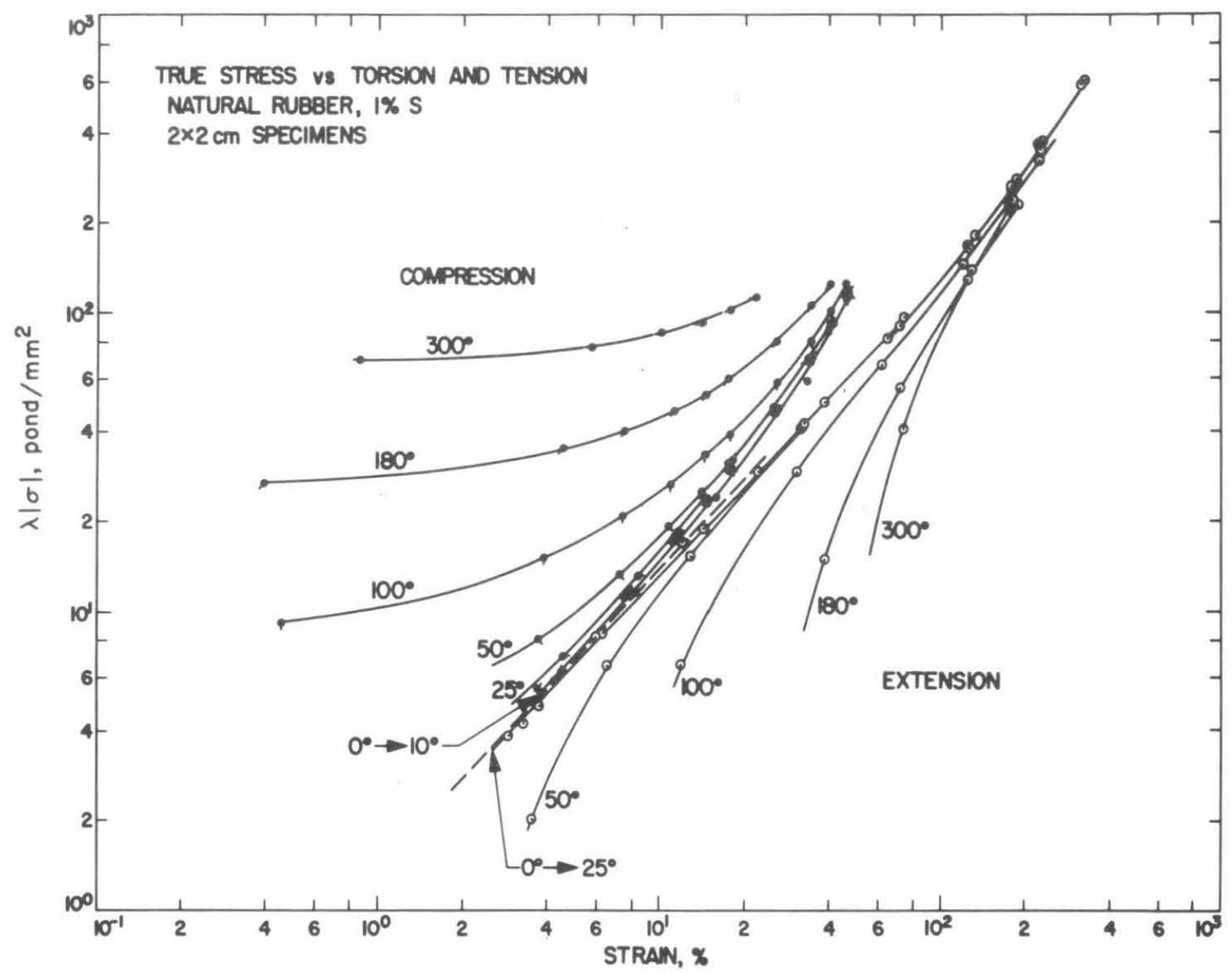

Fig. 3. True Stress vs. Torsion and Tension, Natural Rubber, $1 \%$ S, $2 \times 2$ Specimens. 


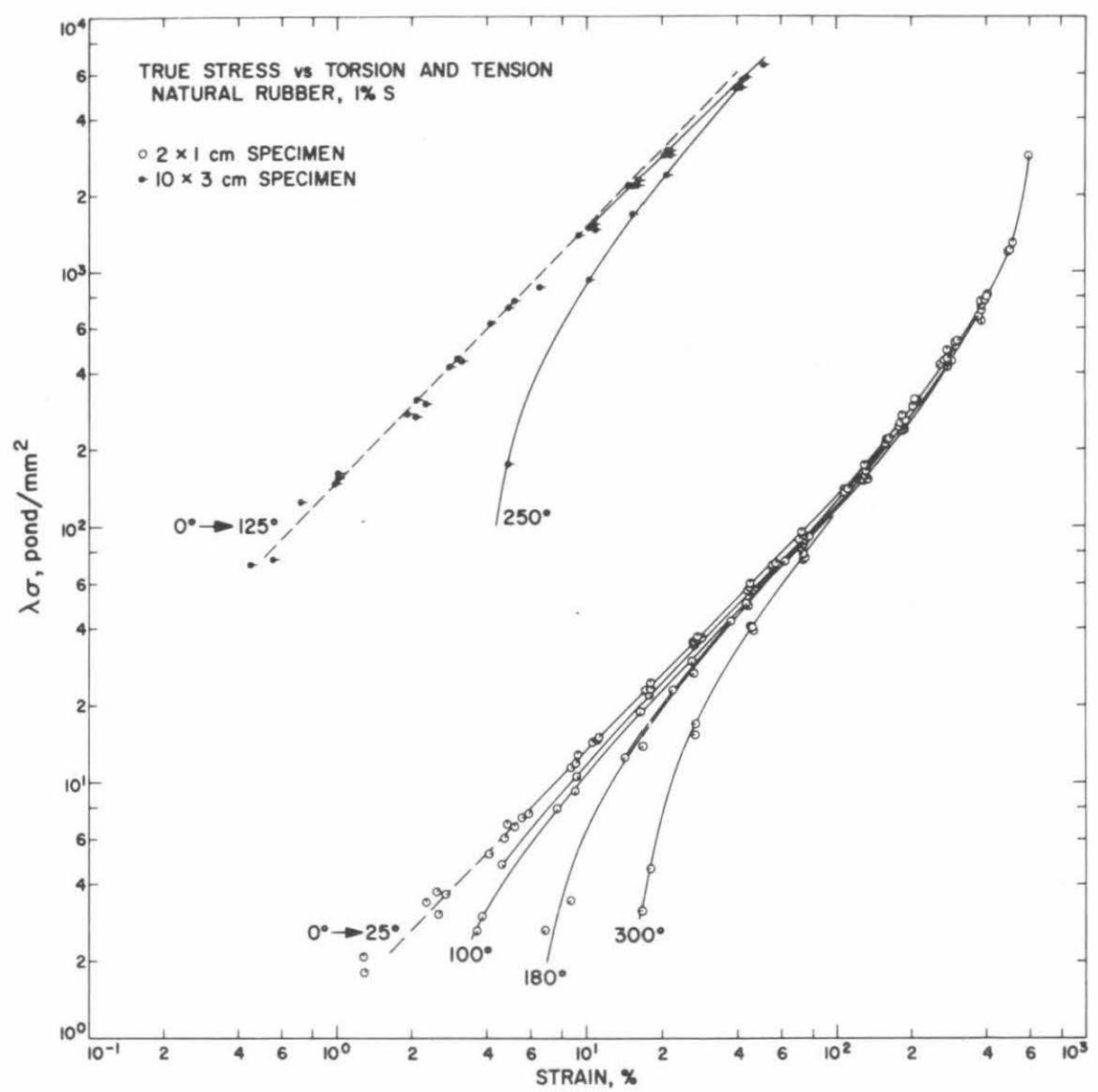

Fig. 4. True Stress vs. Torsion and Tension, Natural Rubber, 1\% S. 
TABLE I

Analysis of the Accumulated Natural Rubber - 14 Sulfur Data Using Equations (5) and (6) All Values are to be Considered Approximate and Temporary, as Discussed in the Text

\begin{tabular}{|c|c|c|c|c|c|c|c|c|c|c|}
\hline \multirow[t]{2}{*}{$\begin{array}{c}\text { Sample } \\
\text { Size }\end{array}$} & \multirow{2}{*}{$\begin{array}{c}\text { Parameter } \\
\text { held } \\
\text { Constant } \\
\end{array}$} & \multirow{2}{*}{$\begin{array}{c}\text { Parameter } \\
\text { Increased } \\
\text { in Steps } \\
\end{array}$} & \multirow[t]{2}{*}{$\begin{array}{l}\text { Test Runs } \\
\text { Made }\end{array}$} & \multirow{2}{*}{$\begin{array}{c}\text { Data } \\
\text { Analyzed }\end{array}$} & \multirow{2}{*}{$\begin{array}{c}\text { Range of } \\
\text { Applicability } \\
\text { of Analysis } \\
\end{array}$} & \multicolumn{5}{|c|}{$\begin{array}{c}\text { Values of Material Parameters, } \\
\text { pond } / \mathrm{mm}^{2}\end{array}$} \\
\hline & & & & & & $\gamma+\beta$ & $y$ & $\beta$ & & $a$ \\
\hline \multicolumn{11}{|c|}{ Data taken in extension: } \\
\hline \multirow[t]{2}{*}{$\begin{array}{l}l_{0}=2 \mathrm{~cm} \\
d_{0}=1 \mathrm{~cm}\end{array}$} & $\underset{(=\lambda-1)}{\varepsilon}$ & $5^{\circ} \stackrel{\theta}{-}-300^{\circ}$ & $\begin{array}{l}14 \text {, at } 14 \\
\text { values of } \\
\varepsilon \text { from } 0.2- \\
5004\end{array}$ & $\mathrm{~N}$ & $\begin{array}{c}\lambda<4^{a, b} \\
c\end{array}$ & $37.5 \pm 2.0$ & $22.0 \pm 1.5$ & $15.5 \pm 2.5$ & $0.4 \pm 0.1$ & \pm 0.1 \\
\hline & $\theta$ & $0-\frac{\varepsilon}{6004}$ & $\begin{array}{l}22, \text { at } 8 \\
\text { values of } \theta \\
\text { from } 0^{\circ}- \\
300^{\circ}\end{array}$ & M & $\lambda<4^{a}$ & $39.5 \pm 3.0$ & $26.0 \pm 5.0$ & $13.5 \pm ?$ & 0.14 & c \\
\hline $\begin{array}{l}l_{0}=2 \mathrm{~cm} \\
d_{0}=2 \mathrm{~cm}\end{array}$ & $\theta$ & $0=3004$ & $\begin{array}{l}7 \text {, at } 7 \\
\text { values of } \theta \\
\text { from } 0^{\circ}- \\
180^{\circ}\end{array}$ & M & $\begin{array}{l}\lambda>1.2 \text { at }^{\mathrm{d}} \\
10^{\circ}, \text { to } \\
\lambda>1.8 \text { at } \\
180^{\circ} \\
c \\
c\end{array}$ & $35 \pm 3$ & $20 \pm 3$ & $15 \pm 3$ & 0.7 & \\
\hline $\begin{array}{l}l_{0}=10 \mathrm{~cm} \\
d_{0}=3 \mathrm{~cm}\end{array}$ & $\theta$ & $\begin{array}{c}c, \\
0-679\end{array}$ & $\begin{array}{l}7 \text {, at } 5 \\
\text { values of } \theta \\
\text { from } 0^{\circ}- \\
250^{\circ}\end{array}$ & M & $\begin{array}{l}\lambda>1.1 \\
\lambda>1.05\end{array}$ & $\begin{array}{r}41 \pm 2 \\
\quad(49) \\
\end{array}$ & $\begin{array}{r}25 \pm 1 \\
28^{c} \\
\end{array}$ & $\begin{array}{r} \pm 3 \\
21^{\mathrm{c}} \\
\end{array}$ & & $\begin{array}{l} \pm ? \\
\pm ? \\
\end{array}$ \\
\hline Data taken & under comp & ression: & & & & & & & & \\
\hline $\begin{array}{l}l_{0}=2 \mathrm{~cm} \\
\mathrm{~d}_{0}=2 \mathrm{~cm}\end{array}$ & $\theta$ & 0 to -508 & $\begin{array}{l}7 \text {, at } 7 \text { values } \\
\text { of } \theta \text { from } 0^{\circ} \text {. } \\
180^{\circ}\end{array}$ & $\begin{array}{l}\mathrm{M} \\
\mathrm{N}\end{array}$ & $\begin{array}{c}\text { All data } \\
\text { c } \\
\end{array}$ & $\begin{array}{r}32 \pm 3 \\
(46) \\
\end{array}$ & $\begin{array}{c}32 \pm 3 \\
55^{\mathrm{c}} \\
\end{array}$ & $\begin{array}{r}0 \pm 1 \\
-9^{c} \\
\end{array}$ & & \\
\hline $\begin{array}{l}\ell_{0}=10 \mathrm{~cm} \\
d_{0}=3 \mathrm{~cm}\end{array}$ & $\theta$ & -2 to -174 & 1 , at $\theta=0^{\circ}$ & $\mathrm{N}$ & All data & (49) & $57^{c}$ & $-8^{c}$ & & $?$ \\
\hline $\begin{array}{ll}\text { a } & \text { Deviat } \\
\text { b } & \text { A diff: } \\
\text { c Analy: } & \text { Anale } \\
\text { d } & \text { See te }\end{array}$ & $\begin{array}{l}\text { tions at high } \\
\text { iculty is disc } \\
\text { sis not comp. } \\
\text { ext. }\end{array}$ & $\begin{array}{l}\text { strains are pr } \\
\text { ussed in the te } \\
\text { eted, and valu }\end{array}$ & $\begin{array}{l}\text { robably due to } c x \\
\text { ext. } \\
\text { ues may apply to }\end{array}$ & $\begin{array}{l}\text { erystallinity } \\
\text { to only one }\end{array}$ & or two tests. & & & & & \\
\hline
\end{tabular}


compression and extension data differ considerably. In extension, the moment data give $\gamma=24 \pm 7, \beta=15 \pm 4$, and $\alpha<1$, whereas moment data in compression give $\gamma=32 \pm 3, \beta=0 \pm 1$, and $\alpha$ small; the Goldberg-Lianis-Signorini-BKZ equations fit either data in extension or compression quite well but completely fail to fit both sets of data together. In extension, our rubber is almost a Mooney material, since $\alpha$ is quite small. In compression, the rubber approximately follows simple kinetic theory behavior, since $\beta$ and $\alpha$ are both about zero.

We do not mean to imply a discontinuity in the $2 \times 2 \mathrm{~cm}$ results at $\lambda=1$. What actually appears to happen is that the best curves in the compression region also fit data at small extensions, up to the region where the compression curves meet the best curves for the larger part of the extension region. (Footnote $\underline{d}$ in the table refers to this fact). Therefore the compression values of $\alpha, \beta$, and $\gamma$ also apply at $\lambda=1$; e.g., the shear modulus at small strains is $\beta+\gamma$ from the compression data, or $32 \pm 3$ pond $/ \mathrm{mm}^{2}$. This behavior of the curves is probably related to the fact that a compressive force develops when the sample is twisted at constant $\lambda$, but more study is needed before conclusions can be drawn.

For the $2 \times 1 \mathrm{~cm}$ samples there were no regions at small $\lambda$ where the data taken at constant $\theta$ and increasing $\lambda$ deviated from the best curves for the extension region. This is in disagreement with the $2 \times 2 \mathrm{~cm}$ results and requires further study. For the test runs at constant $\epsilon$, however, $32 \mathrm{M} / \pi \mathrm{d}^{4} \psi_{\mathrm{d}} \lambda$ often decreased with increasing $\psi_{\mathrm{d}}$, a fact not consistent with Eq. (6) with $\alpha \geq 0$. It is possible that this discrepancy is related to the ideas mentioned just above, The $10 \times 3 \mathrm{~cm}$ data may also exhibit some anomalies very near to $\lambda=1$. 
In the future we will determine the correct $\psi$ values for each specimen size, correct the data and the analysis of them, and prepare a complete report. Then, as soon as the apparatus is rechecked for zero drift and permanent zero shifts, we shall proceed with a similar program of measurements and analysis on the interim standard, GA LCIT I. 


\section{REFERENCES}

1. R. S. Rivlin, in "Rheology," ed. F. R. Eirich, Vol. 1, Academic Press, New York, 1956, pp. 351-385.

2. M. Mooney, J. Appl. Phys., 11, 582 (1940).

3. R. S. Rivlin, Phil. Trans. Roy. Soc. London, A240, 257 (1948).

4. P. J. Blatz and W. L. Ko, Trans. Soc. Rheol., 6, 223 (1962).

5. B. Bernstein, E. A. Kearsley, and L. J. Zapas, Trans. Soc. Rheology., 7, 391 (1963).

6. L. J. Zapas, J. Res. Nat. Bur. Stand., 70A, 525 (1966).

7. G. Lianis, Purdue University Report, AA\&ES 63-11, (1963).

8. K. C. Valanis and R. F. Landel, Trans. Soc. Rheol., 11, 243 (1967).

9. P. Thirion, Cahier Groupe Franc. Etudes Rheol., 5, 137 (1960).

10. R. F. Landel, Jet Propulsion Laboratory Research Summary 36-5, Vol. 2, p. 37, Nov. 1, 1960, Pasadena, Calif.

11. S. Zahorski, Bull. Acad. Polonaise Sci., 10, 421 (1962).

12. A. J. Carmichael and H. W. Holdaway, J. Appl. Phys., 32, 159 (1961).

13. A. Signorini, Ann. Mat. Pura ed Applicata (4), 39, 147 (1955).

14. O. H. Varga, "Stress-Strain Behavior of Elastic Materials," Interscience, New York (1966).

15. A. G. Thomas, Trans. Faraday Soc., 51, 569 (1955).

16. A. N. Gent and A. G. Thomas, J. Polymer Sci., 28, 625 (1958).

17. R. S. Rivlin, in "Viscoelasticity-Phenomenological Aspects, "ed. J. T. Bergen, Academic Press, New York (1960), p. 93.

18. J. T. Bergen, ibid., p. 109.

19. K. C. Valanis and R. F. Landel, J. Appl. Phys., 38, 2997 (1967).

20. A. San Miguel and R. F. Landel, Trans. Soc. Rheol., 10, 369 (1966).

21. G. W. Becker, J. Polymer Sci., C16, 2893 (1967).

22. G. W. Becker, J. M. Kendall, Sr., P. A. Caruthers, and R. F. Landel, Space Programs Summary No. 37-30, Vol. 4, Jet Propulsion Laboratory, Pasadena, California, Nov. 30, 1964, p. 107. 
23. R. S. Rivlin, Phil. Trans. Roy. Soc., A242, 182 (1949).

24. R. F. Fedors and R. F. Landel, Space Programs Summary No. 37-18, Vol. 4, Jet Propulsion Laboratory, Pasadena, California, Dec. 31, 1962 , p. 119.

25. Most of these measurements were made by P. A. Caruthers and H. F. Broyles under the supervision of R. F. Landel, at the Jet Propulsion Laboratory, Pasadena, California.

26. W. Goldberg and G. Lianis, Purdue University Report, AA\&ES 67-6, (1967).

27. L. R. G. Treloar, "The Physics of Rubber Elasticity," Oxford University Press, Oxford, (1958), Chapter 8.

28. R. S. Rivlin and D. W. Saunders, Phil. Trans. Roy. Soc. London, $\underline{A 243}, 251$ (1951).

29. M. J. Forster, J. Appl. Phys., 26, 1104 (1955).

30. G. W. Becker and R. F. Landel, submitted to J. Macromolecular Sci., Physics.

31. W. D. Hutchinson, G. W. Becker, and R. F. Landel, Bulletin of the Fourth Meeting of the Interagency Chemical Rocket Propulsion Group Working Group on Mechanical Behavior, Vol. 1, (1965), p. 141 . 

PART VI

APPROXIMATION METHODS FOR THE DETERMINATION OF THE SPECTRAL DISTRIBUTION FUNCTIONS FROM EXPERIMENTAL RESPONSE FUNCTIONS

N. W. Tschoegl 
PART VI

APPROXIMATION METHODS FOR THE DETERMINATION

OF THE SPECTRAL DISTRIBUTION FUNCTIONS

FROM EXPERIMENTAL RESPONSE FUNCTIONS

\section{CONTENTS}

1. Introduction . . . . . . . . . . . . . . . . VI - 1

2. Approximation Methods to the Relaxation Spectrum from Loss Modulus Data . . . . . . . . . . . V VI - 1

3. Approximations to the Retardation Spectrum from

Storage and Loss Compliance Data . . . . . . . . V VI - 10

4. Stieltjes Transform Inversion. . . . . . . . . . V VI - 13

5. Relaxation Modulus and Creep Compliance . . . . . VI - 17

6. Approximations from Other Viscoelastic Functions . . VI - 24

7. Choice of Approximation Method (with D. G. Fesko . . VI - 25

References . . . . . . . . . . . . . . . . VI - 30 
Fig. 1. The Intensity Functions $I^{\prime \prime} 。(\theta / \tau), I^{\prime \prime}{ }_{1}{ }_{R}(\theta / \tau)$, $I^{\prime \prime} a(\theta / \tau)$, and $I^{\prime \prime}{ }_{a_{R}}(\theta / \tau)$. . . . . . . . . . VI - 3

Fig. 2. The Intensity Functions $I_{1}(\theta / \tau), I_{2}(\theta / \tau)$, and

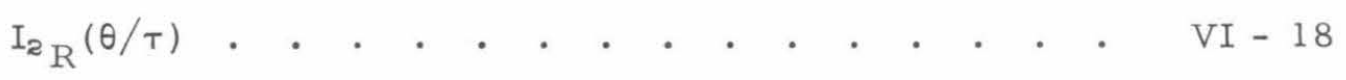

Fig. 3. The Intensity Functions $I_{3}(\theta / \tau), I_{3}(\theta / \tau)$,

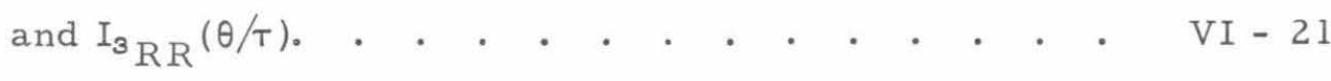

Fig. 4. Dependence of the Approximation on the Slope of the Spectrum . . . . . . . . . . . VI - 27

Fig. 5. $\log H^{\prime}(\tau), \log H_{z}^{\prime}(\sqrt{2} \tau)$, and $\log H_{z}^{\prime}(\tau / \sqrt{2})$ as Functions of $\log \tau^{\circ} \cdot$. . . . . . . . . . VI - 28 


\section{Introduction}

Previously a study was reported ${ }^{(1)}$ of approximation methods for the calculation of the relaxation spectrum from storage modulus data. It was shown that if the order of the highest derivative of the source function in the approximation is $\underline{\mathrm{n}}$, then there exist $\underline{\mathrm{n}}$ approximations to the relaxation spectrum from the storage modulus data. A general method for the derivation of the approximations was developed and several new approximations were found. The relation of these approximations to those in the current literature was investigated, and it was shown that Fujita's approx-

imation $^{(2)}$ is in error because of lack of normalization of the associated intensity function and failure to take into account the necessary shift in the time scale of the relaxation spectrum.

In this report the investigation is extended to approximations to the relaxation spectrum from loss modulus data, approximations to the retardation spectrum from storage compliance and loss compliance data, and approximations to both spectral functions from relaxation modulus and creep compliance data. Approximations to the spectral functions from creep recovery, stress relaxation after cessation of steady flow, and extension at constant rate of strain, were also investigated.

\section{Approximation Methods to the Relaxation Spectrum from}

\section{$\underline{\text { Loss Modulus Data }}$}

The loss modulus is given in terms of the relaxation spectrum $\mathrm{H}(\mathrm{T})$ by

$$
G^{\prime \prime}(\omega)=\int_{0}^{\infty} H(\tau) \frac{\omega \tau}{1+\omega^{2} \tau^{2}} d \ln \tau
$$


$G^{\prime \prime}(\omega)$ is, itself, a crude approximation to the delta function. Following the procedure developed in the previous report ${ }^{(1)}$ we may write

$$
\mathrm{H}^{\prime \prime}{ }_{\mathrm{k}}(\tau)=\theta^{\prime \prime} \mathrm{k}\left[\mathrm{G}^{\prime \prime}(\omega)\right]_{\omega=1 / \tau}
$$

where

$$
\theta^{\prime \prime}{ }_{k}=a_{0}+\sum_{1}^{k} a_{k} \frac{d^{k}}{d l_{n}^{k} \omega}=a_{0}-\sum_{1}^{k} a_{k} \frac{d^{k}}{d l^{k} \tau}
$$

and $\mathrm{k}$ is the order of the approximation.

Applying the operator $\theta^{\prime \prime}{ }_{\mathrm{k}}$ to both sides of Eq. (1) we obtain

$$
H^{\prime \prime}{ }_{k}(\tau)=\int_{0}^{\infty} H(\theta) I^{\prime \prime}{ }_{k}(\theta / \tau) d \ln \theta
$$

where

$$
\mathrm{I}_{\mathrm{k}}^{\prime \prime}(\theta / \mathrm{T})=\theta_{\mathrm{k}}^{\prime \prime}\left[\frac{\theta / \mathrm{T}}{1+\theta^{2} / \mathrm{T}^{2}}\right]
$$

is called the intensity function.

For the zeroth approximation Eq. (5) with $\theta_{0}^{\prime \prime}=a_{0}=a$ yields*

$$
I_{0}^{\prime \prime}(\theta / T)=a \frac{\theta / T}{1+\theta^{2} / T^{2}}
$$

\footnotetext{
* To avoid numerical subscripts, a, b, c, and d will be used for explicit values of $a_{k}$.
} 
The condition that the intensity function be normalized ${ }^{(4)}$

$$
\int_{0}^{\infty} I^{\prime \prime}{ }_{k}(\theta / \tau) d \ln \theta / \tau=1
$$

gives $a=2 / \pi$. We therefore have

$$
I_{0}^{\prime \prime}(\theta / T)=\frac{2 \theta / T}{\pi\left(1+\theta^{2} / T^{2}\right)}
$$

and, from Eq. (2),

$$
\mathrm{H}_{\mathrm{O}}^{\prime \prime}(\tau)=\frac{2}{\pi} \mathrm{G}^{\prime \prime}(\omega)
$$

as required. The intensity function is shown in Fig. 1. It is symmetrical and its maximum occurs at $\ln \theta / T=0$.

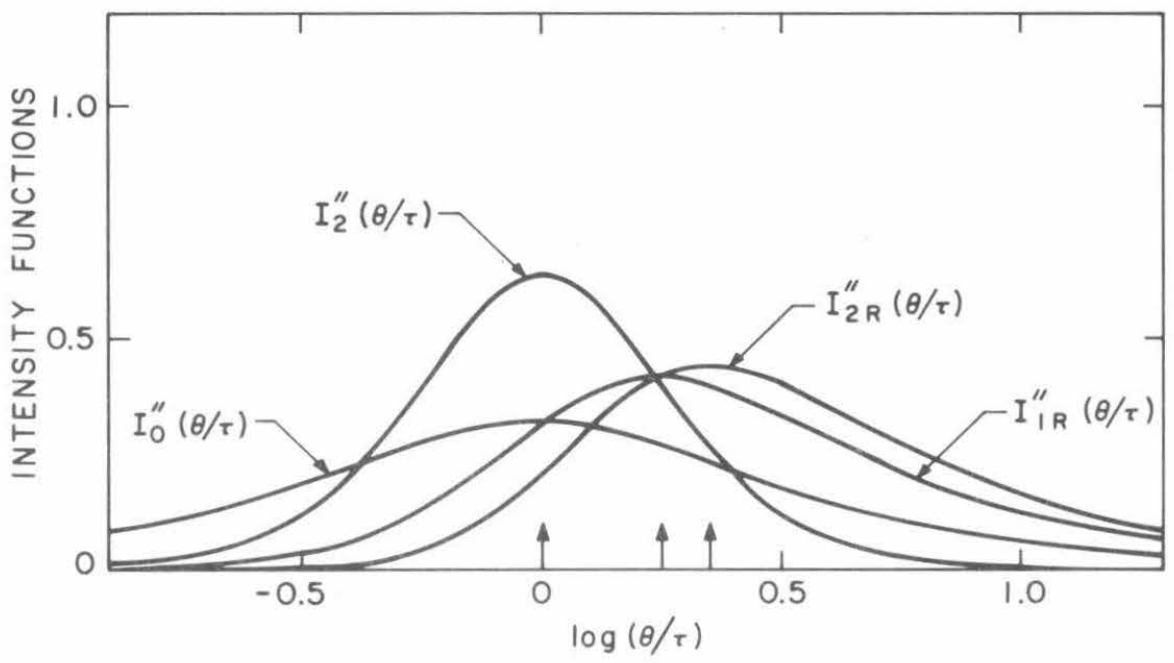

Fig. 1. The Intensity Functions $I_{0}^{\prime \prime}(\theta / \tau)$,

$$
I_{2}^{\prime \prime}(\theta / \tau) \text {, and } I_{2}^{\prime \prime}(\theta / T) \text {. }
$$


The first order approximation $(k=1)$ is found from

$$
\mathrm{H}^{\prime \prime}{ }_{1}(\tau)=\mathrm{a} \mathrm{G}^{\prime \prime}(\omega)+\mathrm{b} \frac{\mathrm{d} \mathrm{G}^{\prime \prime}(\omega)}{\mathrm{d} \ln \omega}
$$

The intensity function becomes

$$
I_{1}^{\prime \prime}(\theta / \tau)=\frac{(a+b) \theta / \tau+(a-b) \theta^{3} / \tau^{3}}{\left(1+\theta^{2} / T^{2}\right)^{2}}
$$

and normalization again yields $a=2 / \pi$. We now apply the condition that the intensity function have a single peak only ${ }^{(1)}$. From

$$
\frac{\mathrm{d} \mathrm{I}_{\mathrm{k}}{ }_{\mathrm{k}}(\theta / \mathrm{T}}{\mathrm{d} \theta / \mathrm{T}} \equiv 0
$$

we obtain

$$
(a-b) \theta^{4} / \tau^{4}+6 b \theta^{2} / \tau^{2}-(a+b)=0
$$

which reduces to an equation with a single positive root for either $a=b$ or $\mathrm{a}=-\mathrm{b}$.

There are, thus, two first order approximations to the relaxation spectrum from loss modulus data. Generally, if the order of the highest derivative, (i.e. the order of the approximation) is $n$, there will be $\mathrm{n}+1$ approximations to the relaxation spectrum from loss modulus data. Substitution of the values for $a$ and $b$ into Eq. (13) shows that the maxima of the two intensity functions occur at $\theta / \tau=1 / \sqrt{3}$ and $\sqrt{3}$ respectively, i. e., to the left and right of $\ln \theta / \tau=0$. From Eq. (11) we obtain 


$$
\begin{aligned}
& I_{1 L}^{\prime \prime}(\theta / T)=\frac{4 \theta / T}{\pi\left(1+\theta^{2} / T^{2}\right)^{2}} \\
& I^{\prime \prime}{ }_{1 R}(\theta / \tau)=\frac{4 \theta^{3} / T^{3}}{\pi\left(1+\theta^{2} / \tau^{2}\right)^{2}}
\end{aligned}
$$

where the subscripts $L$ and $R$ refer to the location of the maxima. The intensity function $I^{\prime \prime}{ }_{R}(\theta / \tau)$ is shown in Fig. 1 . $I^{\prime \prime}{ }_{1}(\theta / \tau)$ is its mirror image and is not shown. The two intensity functions are not symmetric with respect to the intensity axis.

With the appropriate shift in spectrum to take into account the location of the maxima of the intensity functions, the first order approximations then become

$$
\begin{aligned}
& \mathrm{H}^{\prime \prime}{ }_{1}(\tau / \sqrt{3})=\frac{2}{\pi}\left[\mathrm{G}^{\prime \prime}(\omega)+\frac{\mathrm{d} \mathrm{G}^{\prime \prime}(\omega)}{\mathrm{d} \ln \omega}\right]_{\omega=1 / \tau} \\
& \mathrm{H}_{1}^{\prime \prime}(\sqrt{3} \mathrm{\tau})=\frac{2}{\pi}\left[\mathrm{G}^{\prime \prime}(\omega)-\frac{\mathrm{d} \mathrm{G}^{\prime \prime}(\omega)}{\mathrm{d} \ln \omega}\right]_{\left.\right|_{\omega=1 / \tau}}
\end{aligned}
$$

These first order approximations are introduced here for the first time. Their significance lies in the fact that the zeroth approximation is generally too crude, and second, or higher, order approximations are difficult to obtain from experimental data.

To obtain the second order approximations we proceed in an analogous way. We have, for $k=2$,

$$
\mathrm{H}^{\prime \prime}{ }_{2}(\tau)=\mathrm{a} \mathrm{G}^{\prime \prime}(\omega)+\mathrm{b} \frac{\mathrm{d} \mathrm{G}^{\prime \prime} \omega}{\mathrm{d} \ln \omega}+\left.\mathrm{c} \frac{\mathrm{d}^{2} \mathrm{G}^{\prime \prime}(\omega)}{\mathrm{d} \ln ^{2} \omega}\right|_{\omega=1 / \tau}
$$


and find the intensity function to be

$$
I_{2}^{\prime \prime}(\theta / T)=\frac{A \theta / T+B \theta^{3} / T^{3}+C \theta^{5} / T^{5}}{\left(1+\theta^{2} / T^{2}\right)^{3}}
$$

where

$$
\begin{aligned}
& A=a+b+c \\
& B=2(a-3 c) \\
& C=a-b+c
\end{aligned}
$$

Equation (12) now yields

$$
(a-b+c) \theta^{6} / \tau^{6}+(a+5 b-23 c) \theta^{4} / \tau^{4}-(a-5 b-23 c) \theta^{2} / \tau^{2}-(a+b+c)=0
$$

which can be reduced to an equation having only one positive root in three different ways by appropriately equating two of the coefficients of the polynomial to zero.

Solving the resulting three pairs of equations in terms of a gives

$$
\begin{array}{ll}
\mathrm{b}=\frac{4}{3} \mathrm{a}, & \mathrm{c}=\frac{1}{3} \mathrm{a} \\
\mathrm{b}=0, & \mathrm{c}=-\mathrm{a} \\
\mathrm{b}=-\frac{4}{3} \mathrm{a}, & \mathrm{c}=\frac{1}{3} \mathrm{a}
\end{array}
$$

Normalization, as before, yields $a=2 / \pi$. 
The maxima of the intensity functions are located at $\theta / \tau=1 / \sqrt{5}, 1$, and $\sqrt{5}$, and the intensity functions become

$$
\begin{aligned}
& I_{2}^{\prime \prime}(\theta / T)=\frac{16 \theta / T}{3 \pi\left(1+\theta^{2} / T^{2}\right)^{3}} \\
& I_{2}^{\prime \prime}(\theta / \tau)=\frac{16 \theta^{3} / T^{3}}{\pi\left(1+\theta^{2} / T^{2}\right)^{3}} \\
& I^{\prime \prime}{ }_{R}(\theta / T)=\frac{16 \theta^{5} / \tau^{5}}{3 \pi\left(1+\theta^{2} / T^{2}\right)^{3}}
\end{aligned}
$$

The last two functions are shown in Fig. 1. Equation (2lb) is, of course, the intensity function associated with the approximation of Schwarzl and Staverman ${ }^{(4)}$. Its maximum occurs at $\ln \theta / \mathrm{T}=0$ and it is symmetric with respect to the intensity axis. The other two intensity functions are again mirror images of each other. They are not symmetrical, and their peaks lie to the left and right of the origin.

The corresponding second order approximations become

$$
\begin{aligned}
& \mathrm{H}_{2}^{\prime \prime}(\tau / \sqrt{5})=\frac{2}{\pi}\left[\mathrm{G}^{\prime \prime}(\omega)+\frac{4}{3} \frac{\mathrm{d} \mathrm{G}^{\prime \prime}(\omega)}{\mathrm{d} \ln \omega}+\frac{1}{3} \frac{\mathrm{d}^{2} \mathrm{G}^{\prime \prime}(\omega)}{\mathrm{d} \ln \mathrm{n}^{2} \omega}\right]_{\omega=1 / \tau} \\
& \mathrm{H}_{2}^{\prime \prime}(\tau)=\frac{2}{\pi}\left[\mathrm{G}^{\prime \prime}(\omega)-\frac{\mathrm{d}^{2} \mathrm{G}^{\prime \prime}(\omega)}{\mathrm{d} \ln ^{2} \omega}\right]_{\omega=1 / \tau} \\
& \mathrm{H}_{2}^{\prime \prime}(\sqrt{5} \tau)=\frac{2}{\pi}\left[\mathrm{G}^{\prime \prime}(\omega)-\frac{4}{3} \frac{\mathrm{d} \mathrm{G}^{\prime \prime}(\omega)}{\mathrm{d} \ln \omega}+\frac{1}{3} \frac{\mathrm{d}^{2} \mathrm{G}^{\prime \prime}(\omega)}{\mathrm{d}^{2} \ln { }^{2}(\omega)}-\left.\right|_{\omega=1 / \tau}\right.
\end{aligned}
$$


For the approximations of third order $(k=3)$ we start from

$$
\mathrm{H}^{\prime \prime}{ }_{3}(\mathrm{\tau})=\mathrm{a} \mathrm{G}^{\prime \prime}(\omega)+\mathrm{b} \frac{\mathrm{d} \mathrm{G}^{\prime \prime}(\omega)}{\mathrm{d} \ln \omega}+\mathrm{c} \frac{\mathrm{d}^{2} \mathrm{G}^{\prime \prime}(\omega)}{\mathrm{d} \ln ^{2} \omega}+\left.\mathrm{d} \frac{\mathrm{d}^{3} \mathrm{G}^{\prime \prime}(\omega)}{\mathrm{d} \ln ^{3} \omega}\right|_{\omega=1 / \tau}
$$

and obtain

$$
I^{\prime \prime}{ }_{3}(\theta / T)=\frac{A \theta / T+B \theta^{3} / T^{3}+C \theta^{5} / T^{5}+D \theta^{7} / T^{7}}{\left(1+\theta^{2} / T^{2}\right)^{4}}
$$

where

$$
\begin{aligned}
& A=a+b+c+d \\
& B=3 a+b-5 c-23 d \\
& C=3 a-b-5 c+23 d \\
& D=a-b+c-d
\end{aligned}
$$

The single peak requirement yields

$$
\begin{aligned}
(a-b+c-d) \theta^{8} / \tau^{8} & -2(a+2 b-11 c+38 d) \theta^{6} / T^{6}+10(b-23 d) \theta^{4} / \tau^{4} \\
& -2(a-2 b-11 c-38 d) \theta^{2} / T^{2}-(a+b+c+d)=0
\end{aligned}
$$

To obtain an equation with a single positive root, we equate appropriately selected coefficients in Eq. (26) to zero. Solving the resulting four sets of three equations each for $\underline{b}, \underline{c}$, and $\underline{d}$ in terms of $\underline{\text { a }}$ leads to either 

$b=23 a / 15$,
$c=3 a / 5$,
$\mathrm{d}=\mathrm{a} / 15$
or
$b=a / 3$,
$c=-a$,
$\mathrm{d}=-\mathrm{a} / 3$
$\mathrm{b}=-\mathrm{a} / 3$,
$c=-a$,
$\mathrm{d}=\mathrm{a} / 3$
$\mathrm{b}=-23 \mathrm{a} / 15$,
$c=3 a / 5$,
$\mathrm{d}=-\mathrm{a} / 15$.

or

or

Normalization yields $a=2 / \pi$ for the first and last group of coefficients, and $a=2 / 3 \pi$ for the second and third. The four intensity functions become

$$
\begin{aligned}
I^{\prime \prime}{ } L_{L}(\theta / T) & =\frac{32 \theta / T}{5 \pi\left(1+\theta^{2} / T^{2}\right)^{4}} \\
I^{\prime \prime}{ }_{3} L^{(\theta / T)} & =\frac{32 \theta^{3} / T^{3}}{3 \pi\left(1+\theta^{2} / T^{2}\right)^{4}} \\
I^{\prime \prime}{ }_{3}{ }^{(\theta / T)} & =\frac{32 \theta^{5} / T^{5}}{3 \pi\left(1+\theta^{2} / T^{2}\right)^{4}} \\
I^{\prime \prime}{ }^{3}{ }_{R R}(\theta / T) & =\frac{32 \theta^{7} / T^{7}}{5 \pi\left(1+\theta^{2} / T^{2}\right)^{4}}
\end{aligned}
$$

Double subscripts indicate that the intensity functions are farthest to the left or right respectively. The maxima occur at $\theta / \tau=1 / \sqrt{7}, 1 / \sqrt{5 / 3}$, $\sqrt{5 / 3}$, and $\sqrt{7}$. There is thus no third order intensity function which is symmetric with respect to the intensity axis and whose maximum occurs at $\ln \theta / T=0$. 
The four approximations now become

$$
\mathrm{H}^{\prime \prime}{ }_{3}(\tau / \sqrt{ } 7)=\frac{2}{\pi}\left[\mathrm{G}^{\prime \prime}(\omega)+\frac{23}{15} \frac{\mathrm{d} \mathrm{G}^{\prime \prime}(\omega)}{\mathrm{d} \ln \omega}+\frac{3}{5} \frac{\mathrm{d}^{2} \mathrm{G}^{\prime \prime}(\omega)}{\mathrm{d} \ln ^{2} \omega}+\frac{1}{15} \frac{\mathrm{d}^{3} \mathrm{G}^{\prime \prime}(\omega)}{\mathrm{d} \ln ^{3} \omega}\right]_{\omega=1 / \tau}
$$

$$
\mathrm{H}^{\prime \prime}{ }_{3}(\tau / \sqrt{5 / 3})=\frac{2}{3 \pi}\left[\mathrm{G}^{\prime \prime}(\omega)+\frac{1}{3} \frac{\mathrm{d} \mathrm{G}^{\prime \prime}(\omega)}{\mathrm{d} \ln \omega}-\frac{\mathrm{d}^{2} \mathrm{G}^{\prime \prime}(\omega)}{\mathrm{d} \ln ^{2} \omega}-\frac{1}{3} \frac{\mathrm{d}^{3} \mathrm{G}^{\prime \prime}(\omega)}{\mathrm{d} \ln ^{3} \omega}\right]_{\left.\right|_{\omega=1 / \tau}}
$$

$$
\mathrm{H}^{\prime \prime}{ }_{3}(\sqrt{5 / 3} \tau)=\frac{2}{3 \pi}\left[\mathrm{G}^{\prime \prime}(\omega)-\frac{1}{3} \frac{\mathrm{d} \mathrm{G}^{\prime \prime}(\omega)}{\mathrm{d} \ln \omega}-\frac{\mathrm{d}^{2} \mathrm{G}^{\prime \prime}(\omega)}{\mathrm{d} \ln ^{2} \omega}+\frac{1}{3} \frac{\mathrm{d}^{3} \mathrm{G}^{\prime \prime}(\omega)}{\mathrm{d} \ln ^{3} \omega}\right]_{\omega=1 / \tau}
$$

$$
\mathrm{H}^{\prime \prime}{ }_{3}(\sqrt{7} \mathrm{\tau})=\frac{2}{\pi}\left[\mathrm{G}^{\prime \prime}(\omega)-\frac{23}{15} \frac{\mathrm{d} \mathrm{G}^{\prime \prime}(\omega)}{\mathrm{d} \ln \omega}+\frac{3}{5} \frac{\mathrm{d}^{2} \mathrm{G}^{\prime \prime}(\omega)}{\mathrm{d} \ln ^{2} \omega}-\frac{1}{15} \frac{\mathrm{d}^{3} \mathrm{G}^{\prime \prime}(\omega)}{\mathrm{d} \ln ^{3} \omega}\right]_{\omega=1 / \tau}
$$

\section{Approximations to the Retardation Spectrum from Storage and}

\section{Loss Compliance Data}

Extension of the procedure used for obtaining approximations to the relaxation spectrum from storage and loss moduli to the retardation spectrum from compliance data is easily accomplished. The storage compliance is given by

$$
J^{\prime}(\omega)=J_{g}+\int_{0}^{\infty} L(\tau) \frac{1}{1+\omega^{2} \tau^{2}} d \ln \tau
$$


where $\mathrm{J}_{\mathrm{g}}$ is the glass compliance, and $\mathrm{L}(\tau)$ is the retardation spectrum. We have

$$
L_{k}^{\prime}(\tau)=\theta_{k}^{\prime}\left[J^{\prime}(\omega)-J_{g}\right]_{\omega=1 / \tau}=\left.\theta^{\prime}\left[J^{\prime}(\omega)\right]\right|_{\omega=\tau}
$$

where

$$
\theta_{\mathrm{k}}^{\prime}=\sum_{1}^{\mathrm{k}} \mathrm{a}_{\mathrm{k}} \frac{\mathrm{d}^{\mathrm{k}}}{\mathrm{d} \mathrm{ln}^{\mathrm{k}} \omega}=-\sum_{1}^{\mathrm{k}} \mathrm{a}_{\mathrm{k}} \frac{\mathrm{d}^{\mathrm{k}}}{\mathrm{d} \mathrm{ln}^{\mathrm{k}} \tau}
$$

The intensity function becomes

$$
I_{k}^{\prime}(\theta / \tau)=\theta_{k}^{\prime}\left[\frac{1}{1+\theta^{2} / \tau^{2}}\right]
$$

But

$$
\frac{d}{d \ln \tau}\left[\frac{1}{1+\theta^{2} \tau^{2}}\right]=-\frac{d}{d \ln \tau}\left[\frac{\theta^{2} / \tau^{2}}{1+\theta^{2} / \tau^{2}}\right]
$$

and hence

$$
\theta_{\mathrm{k}}^{\prime}\left[\frac{1}{1+\omega^{2} \tau^{2}}=-\theta_{\mathrm{k}}^{\prime}\left[\frac{\omega^{2} T^{2}}{1+\omega^{2} \tau^{2}}\right]\right.
$$

It follows from Eq. (34) that the storage compliance approximations to the retardation spectrum and the storage modulus approximations to the relaxation spectrum have identical intensity functions, and are identical in form except that the storage compliance approximations have the negative sign. As an example, the two second order approximations are 


$$
\begin{aligned}
& L_{a}^{\prime}(\tau / \sqrt{2})=-\left.\left[\frac{\mathrm{d} J^{\prime}(\omega)}{d \ln \omega}+\frac{1}{2} \frac{d^{2} J^{\prime}(\omega)}{d \ln ^{2} \omega}\right]\right|_{\omega=1 / \tau} \\
& L_{2}^{\prime}(\sqrt{2} \tau)=-\left[\frac{d J^{\prime}(\omega)}{d \ln \omega}-\frac{1}{2} \frac{d^{2} J^{\prime}(\omega)}{d \ln ^{2} \omega}\right]_{\omega=1 / \tau}
\end{aligned}
$$

Turning now to the loss compliance, we have

$$
J^{\prime \prime}(\omega)=\int_{0}^{\infty} L(\tau) \frac{\omega \tau}{1+\omega^{2} \tau^{2}} d \ln \tau+\left\{\frac{1}{\omega \eta}\right\}
$$

where $\eta$ is the steady-flow viscosity, and we find

$$
L^{\prime \prime}{ }_{k}(\tau)=\theta^{\prime \prime}\left[J^{\prime \prime}(\omega)-\{1 / \omega \eta\}\right]
$$

where $\theta_{k}^{\prime \prime}$ is given by Eq. (3), and the braces signify that $1 / \omega \eta$ is to be omitted if the material is crosslinked.

The intensity function becomes

$$
I^{\prime \prime}{ }_{k}(\theta / \tau)=\theta^{\prime \prime}\left[\frac{\theta / \tau}{1+\theta^{2} / T^{2}}\right]
$$

and is thus identical with the intensity function for the approximations derived from the storage modulus. This is, of course, a consequence of the identity of the kernels in Eqs. (1) and (36). The approximations to the retardation spectrum from loss compliance data are therefore identical with those for the relaxation spectrum from the loss modulus, except that the flow term, $1 / \omega \eta$, must be taken into account when applicable. 


\section{Stieltjes Transform Inversion}

It was shown in the previous report ${ }^{(1)}$ that the relation between the storage modulus and the relaxation spectrum,

$$
G^{\prime}(\omega)=\left\{G_{e}\right\}+\int_{0}^{\infty} H(\tau) \frac{\omega^{2} \tau^{2}}{1+\omega^{2} \tau^{2}} d \ln \tau
$$

(where the braces signify that the equilibrium modulus, $G_{e}$, is to be omitted if the material is not crosslinked), could, with an appropriate change in variables, be brought into the form of the Stieltjes transform

$$
f(x)=\int_{0}^{\infty} \frac{\Phi(t)}{x+t} d t
$$

to which known inversion formulae can be applied. It was shown that the inversion formulae yielded the approximations corresponding to the intensity functions $I^{\prime}{ }_{2}(\theta / \tau)$ and $I^{\prime}{ }_{3}(\theta / \tau)$ as the two lowest approximations obtainable by the transform inversion method. It should be noted that the approximation of Schwarzl and Staverman with intensity function $I^{\prime}{ }_{3}(\theta / \tau)$ could not be derived by this method.

In this section we now apply the Stieltjes transform inversion method to the loss modulus, and to the storage and loss compliance. For the los s modulus, Eq. (1) can be brought into the form of Eq. (40) with the change of variables $\tau^{2}=t, \omega^{2}=1 / x$. We then have

$$
f(x)=\left.2 \omega G^{\prime \prime}(\omega)\right|_{\omega=1 / \sqrt{x}}
$$

and

$$
\Phi(t)=H(\tau) /\left.\tau\right|_{\tau=\sqrt{t}}
$$


Inversion of the transform will yield only approximations of order two or higher (cf. previous Annual Report ${ }^{(1)}$ ). The inversion equation of Hirschman and Widder ${ }^{(1)}$

$$
\Phi_{a_{n}}(t)=\left.\frac{(-1)^{n}}{2 \pi}\left(\frac{e}{n}\right)^{2 n} \frac{d^{n}}{d x^{n}}\left[x^{2 n} \frac{d^{n}}{d x^{n}} f(x)\right]\right|_{x=t}
$$

yields

$$
\Phi_{2}(t)=-\left.\frac{e^{2}}{2 \pi}\left[2 x \frac{d}{d x}+x^{2} \frac{d^{2}}{d x^{2}}\right] f(x)\right|_{x=t}
$$

for $n=1$, where $2 n=k$, and $k$ is the index used earlier to denote the order of the approximation. With the appropriate resubstitutions using Eqs. (41) and (42) this leads to Fujita's approximation

$$
\mathrm{H}_{\mathrm{F}}^{\prime \prime}(\tau)=\left.\frac{\mathrm{e}^{2}}{4 \pi}\left[\mathrm{G}^{\prime \prime}(\omega)-\frac{\mathrm{d}^{2} \mathrm{G}^{\prime \prime}(\omega)}{\mathrm{d} \ell_{\mathrm{n}^{2} \omega}}\right]\right|_{\omega=1 / \tau}
$$

with the intensity function

$$
I_{F}^{\prime \prime}(\theta / T)=\frac{2 e^{2} \theta^{2} / T^{3}}{\pi\left(1+\theta^{2} / T^{2}\right)^{3}}
$$

which is not normalized. The normalization factor, $8 / \mathrm{e}^{2}$, restores Fujita's approximation to the approximation of Schwarzl and Staverman.

For the third order approximation the equation of Widder ${ }^{(1)}$

$$
\Phi_{(2 n+1)}(t)=\left.\frac{(-1)^{n}}{(n+1) !(n-1) !} \frac{d^{n+1}}{d x^{n+1}}\left[x^{2 n+1} \frac{d^{n}}{d x^{n}} f(x)\right]\right|_{x=t}
$$


yields, with $\mathrm{n}=1,2 \mathrm{n}+\mathrm{l}=\mathrm{k}$, the non-normalized intensity function

$$
I_{3}^{\prime \prime}(\theta / T)=\frac{6 \theta^{5} / T^{5}}{\left(1+\theta^{2} / T^{2}\right)^{4}}
$$

and, with the appropriate shift in the time scale of the spectrum

$$
\mathrm{H}^{\prime \prime}{ }_{3}(\sqrt{5 / 3} \mathrm{~T})=\frac{3}{8}\left[\mathrm{G}^{\prime \prime}(\omega)-\frac{1}{3} \frac{\mathrm{d} \mathrm{G}^{\prime \prime}(\omega)}{\mathrm{d} \ln \omega}-\frac{\mathrm{d}^{2} \mathrm{G}^{\prime \prime}(\omega)}{\mathrm{d} \ln ^{2} \omega}+\frac{1}{3} \frac{\mathrm{d}^{3} \mathrm{G}^{\prime \prime}(\omega)}{\mathrm{d} \ln { }^{3} \omega}\right]_{\omega=1 / \tau}
$$

The normalization factor, $16 / 3 \pi$, reduces Eq. (49) to Eq. (28c).

As in the case of the storage modulus, inversion of the Stieltjes transform yields only one of the approximations of higher order. In the case of the storage modulus the Stieltjes transform inversion yielded the approximations with normalized intensity functions. However, in the case of the loss modulus an approximation is obtained whose intensity function is not normalized; and the correct form of the approximation can only be found after normalization.

For the loss compliance, Eq. (40) is obtained from Eq. (36) with

$$
f(x)=2 \omega J^{\prime \prime}(\omega)-\left.\{1 / \omega \eta\}\right|_{\omega=1 / \sqrt{x}}
$$

and

$$
\Phi(t)=L(\tau) /\left.\tau\right|_{T=\sqrt{t}}
$$

The approximations and intensity functions furnished by the transform inversion method will therefore be simply the analogs of those obtained from the loss modulus. 
For the storage compliance, however, the situation is different. Equation (29) gives Eq. (40) with

$$
\begin{aligned}
& f(x)=\left.2 \omega^{2} J^{\prime}(\omega)\right|_{\omega=1 / \sqrt{x}} \\
& \Phi(t)=L(\tau) /\left.\tau^{2}\right|_{\tau=\sqrt{t}}
\end{aligned}
$$

For the second order approximation we thus obtain (with the appropriate shift of the spectrum)

$$
L_{a}^{\prime}(\tau / \sqrt{2})=-\left[\frac{\mathrm{d} J^{\prime}(\omega)}{\mathrm{d} \ln \omega}+\frac{1}{2} \frac{\mathrm{d}^{2} \mathrm{~J}^{\prime}(\omega)}{\mathrm{d} \ln \mathrm{n}^{2} \omega}\right]
$$

It should be noted that for the storage modulus the same procedure yielded $\mathrm{H}^{\prime}{ }_{2}(\sqrt{2} \mathrm{~T})$.

For the third order approximation Eqs. (52) and (53) yield

$$
L_{3}^{\prime}(\tau)=-\frac{1}{2}\left[\frac{d J^{\prime}(\omega)}{d \ln \omega}-\frac{1}{4} \frac{d^{3} J^{\prime}(\omega)}{d \ln ^{3} \omega}\right]
$$

The corresponding intensity function is not normalized. After normalization by the factor 2, Eq. (55) becomes identical with the approximation of Schwarzl and Staverman ${ }^{(4)}$. In the case of the storage modulus the same procedure had yielded $\mathrm{H}_{3}{ }_{3}(\sqrt{3} \mathrm{~T})$.

It is thus clear that the Stieltjes transform inversion method does not lead to corresponding equations when used to derive approximations from the two storage functions. 


\section{Relaxation Modulus and Creep Compliance}

Approximations to the relaxation and retardation spectra have been given by Schwarzl and Staverman ${ }^{(1)}$. It will be shown here, however, that these are not the only possible ones. The general method which has been applied to the dynamic response functions in the preceeding pages, may equally be applied to the transient functions also. It will be shown that here again $k$ approximations of order $k$ exist.

For the relaxation modulus we have

$$
G(t)=\left\{G_{e}\right\}+\int_{0}^{\infty} H(\tau) \exp -t / \tau \cdot d \ln \tau
$$

where the braces have their previous significance. Proceeding as before we have

$$
H_{k}(\tau)=\theta_{k}\left[G(t)-\left\{G_{e}\right\}\right]_{t=\tau}=\theta_{k}[G(t)]_{t=\tau}
$$

where

$$
\theta_{k}=\sum_{1}^{k} a_{k} \frac{d^{k}}{d \ell n^{k} t}=\sum_{1}^{k} a_{k} \frac{d^{k}}{d \ln ^{k} \tau}
$$

Changing the variable of integration to $\theta$, then setting $t=\tau$, and substituting Eq. (56) into (57) gives

$$
H_{k}(\tau)=\int_{0}^{\infty} H(\theta) I_{k}(\theta / \tau) d \ln \theta
$$


where

$$
I_{k}(\theta / \tau)=\theta_{k}[\exp -\tau / \theta]
$$

is the intensity function.

We obtain the first order approximation with $k=1$ as

$$
H_{1}(\tau)=\left.a \frac{d G(t)}{d \ln t}\right|_{t=\tau}
$$

which has the intensity function

$$
I_{1}(\theta / \tau)=-\frac{a}{\theta / \tau} \exp -\tau / \theta
$$

Normalization yields $a=-1$. The intensity function has a single maximum at $\ln \theta / T=0$ but it is not symmetrical with respect to the intensity axis as shown in Fig. 2 .

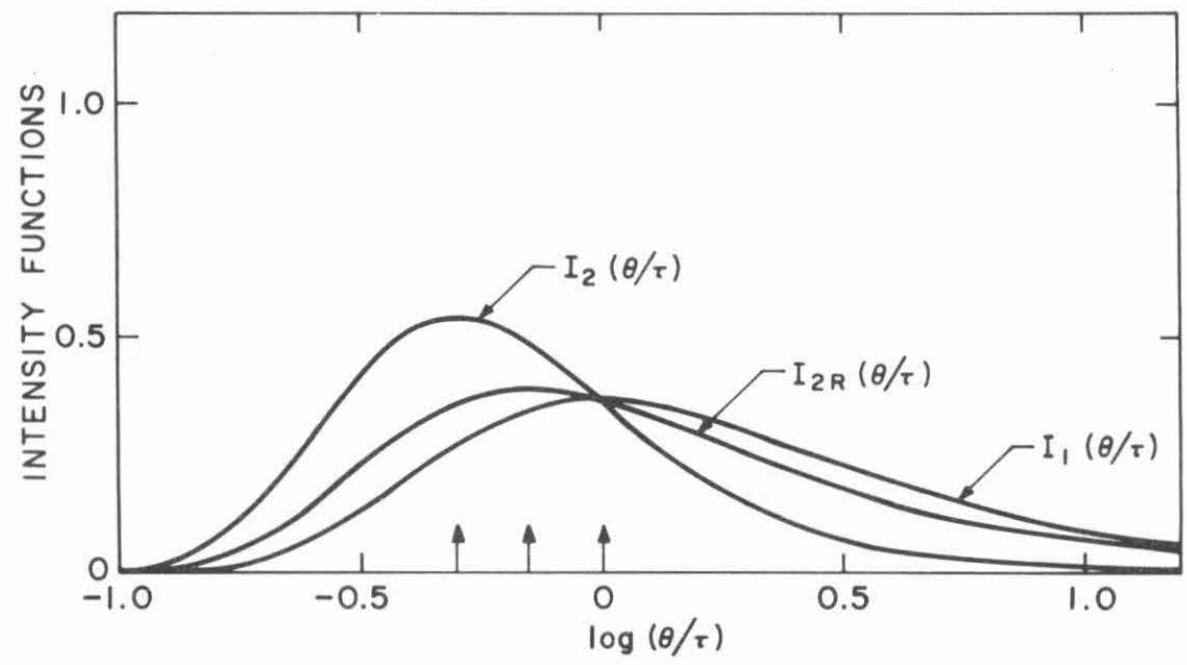

Fig. 2. The Intensity Functions $I_{1}(\theta / \tau), I_{2}(\theta / \tau)$, and $I_{z_{R}}(\theta / \tau)$. 
For the second order approximation $(k=2)$ we have

$$
H_{2}(\tau)=a \frac{d G(t)}{d \ln t}+\left.b \frac{d^{2} G(t)}{d \ln ^{2} t}\right|_{t=\tau}
$$

with intensity function

$$
I_{2}(\theta / T)=-\left[\frac{a+b}{\theta / T}-\frac{b}{\theta^{2} / T^{2}}\right] \exp -\tau / \theta
$$

This will have a single maximum if $d I_{2}(\theta / \tau) / d \theta / T=0$ has a single positive root. The differentiation yields

$$
(a+b)(\theta / \tau)^{2}-(a+3 b) \theta / \tau+b=0
$$

If $I_{2}(\theta / \tau)$ is to have a single peak only we must have either $b=-a$ or $\mathrm{b}=-\mathrm{a} / 3$. Normalization yields $\mathrm{a}=-1$ in both cases and the intensity functions become

$$
I_{2}(\theta / \tau)=(\tau / \theta)^{2} \exp -\tau / \theta
$$

and

$$
I_{2}(\theta / T)=\frac{2 \theta / T+1}{3 \theta^{2} / T^{2}} \exp -\tau / \theta
$$

As shown in Fig. 2 neither of the intensity functions is symmetrical. In the first case the maximum occurs when $\theta / \tau=\frac{1}{2}$, in the second when $\theta / \tau=1 / \sqrt{2}$. With the appropriate shift in the time scale of the spectrum the approximations become 


$$
H_{2}(\tau / 2)=-\frac{d G(t)}{d \ln t}+\left.\frac{d^{2} G(t)}{d \ln ^{2} t}\right|_{t=\tau}
$$

and

$$
H_{2}(\tau / \sqrt{2})=-\frac{d G(t)}{d \ln t}+\left.\frac{1}{3} \frac{d^{2} G(t)}{d \ln ^{2} t}\right|_{t=\tau}
$$

The first of these is the well-known approximation of Schwarzl and Staverman (4). The second is a new approximation.

Proceeding similarly for $\mathrm{k}=3$, we have

$$
H_{3}(\tau)=a \frac{d G(t)}{d \ln t}+b \frac{d^{2} G(t)}{d \ln ^{2} t}+\left.c \frac{d^{3} G(t)}{d \ln ^{3} t}\right|_{t=\tau}
$$

with the intensity function

$$
I_{3}(\theta / \tau)=-\left[\frac{a+b+c}{\theta / \tau}-\frac{b+3 c}{\theta^{2} / \tau^{2}}+\frac{c}{\theta^{3} / \tau^{3}}\right] \exp -\tau / \theta
$$

Differentiation yields

$$
(a+b+c) \theta^{3} / T^{3}-(a+3 b+7 c) \theta^{2} / \tau^{2}+(b+6 c) \theta / \tau-c=0
$$

which will fulfill the conditions of a single maximum when either

or

$$
\mathrm{b}=-3 \mathrm{a} / 2, \quad \mathrm{c}=\mathrm{a} / 2
$$

or

$$
\mathrm{b}=-6 \mathrm{a} / 5, \quad \mathrm{c}=\mathrm{a} / 5
$$

$$
\mathrm{b}=-6 \mathrm{a} / 11 \text {, }
$$$$
c=a / 11 \text {. }
$$

The intensity functions become, with $a=-1$ obtained from normalization, 


$$
\begin{aligned}
& I_{3}(\theta / T)=\frac{1}{2 \theta^{3} / T^{3}} \exp -\tau / \theta \\
& I_{3}(\theta / T)=\frac{3 \theta / T+1}{5 \theta^{3} / T^{3}} \exp -\tau / \theta
\end{aligned}
$$

and

$$
I_{3_{R R}}(\theta / T)=\frac{6 \theta^{2} / T^{2}+3 \theta / T+1}{11 \theta^{3} / T^{3}}
$$

and the maxima are located at $\theta / \tau=1 / 3,1 / \sqrt{6}$, and $1 / \sqrt[3]{6}$ as shown in Fig. 3 .

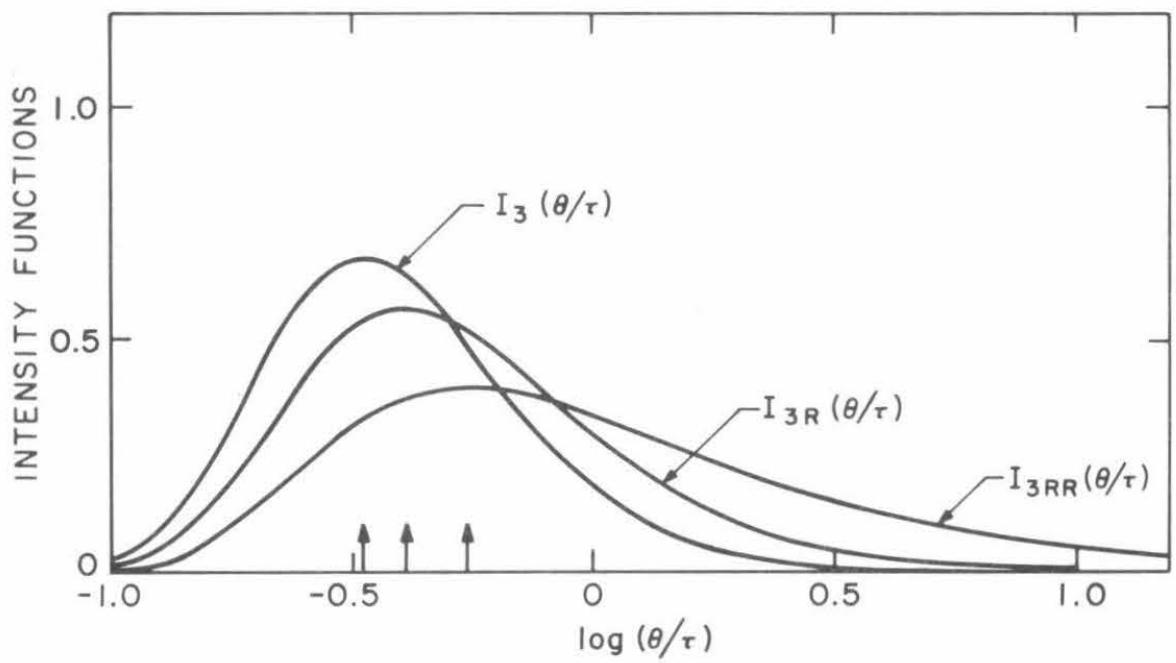

Fig. 3. The Intensity Functions $I_{3}(\theta / \tau), I_{3}(\theta / \tau)$, and $I_{3} R R(/ \tau)$.

The three second order approximations are

$$
H(\tau / 3)=-\frac{d G(t)}{d \ln t}+\frac{3}{2} \frac{d^{2} G(t)}{d \ln ^{2} t}-\left.\frac{1}{2} \frac{d^{3} G(t)}{d \ln ^{3} t}\right|_{t=\tau}
$$




$$
\begin{aligned}
& H(\tau / \sqrt{6})=-\frac{d G(t)}{d \ln t}+\frac{6}{5} \frac{d^{2} G(t)}{d \ln ^{2} t}-\left.\frac{1}{5} \frac{d^{3} G(t)}{d \ln ^{3} t}\right|_{t=\tau} \\
& H(\tau / \sqrt[3]{6})=-\frac{d G(t)}{d \ln t}+\frac{6}{11} \frac{d^{2} G(t)}{d \ln ^{2} t}-\left.\frac{1}{11} \frac{d^{3} G(t)}{d \ln ^{3} t}\right|_{t=\tau}
\end{aligned}
$$

Equation (72a) is, of course, the approximation of Schwarzl and Staverman ${ }^{(4)}$. As shown by Schwarzl and Staverman ${ }^{(4)}$ Eq. (56) can be brought into the form of the Laplace transform with the change of variables $\tau=1 / \zeta$

$$
f(t)=\int_{0}^{\infty} \varphi(\zeta) \exp -t \zeta \cdot d \zeta
$$

where

$$
\begin{aligned}
& f(t)=G(t)-\left\{G_{e}\right\} \\
& \varphi(\zeta)=\left.\tau H(\tau)\right|_{\tau=1 / \zeta}
\end{aligned}
$$

The Post-Widder inversion formula ${ }^{(5)}$

$$
\varphi_{k}(\zeta)=\frac{(-1)^{k}}{(k-1) !} \frac{t}{k} \frac{d^{k}}{d t^{k}} f(t) \mid t=k / \zeta
$$

then yields Eqs. (61), (67a) and (72a) for $k=1,2$, and 3 respectively. In all cases the intensity functions associated with the approximation derived by the transform inversion method are normalized. The new approximations, Eqs. (67b), and (72b) and (72c), contain smaller contributions from the higher derivatives than do the approximations of Schwarzl and Staverman 
and require smaller shifts in the time scale of the spectrum. However, as shown in Figs. 2 and 3, the associated intensity functions are broader.

Applying the same method to the creep compliance

$$
J(t)=J_{g}+\int_{0}^{\infty} L(\tau)[1-\exp -t / \tau] d \ln \tau+t / \eta
$$

the approximations to the retardation spectrum are obtained from

$$
\mathrm{L}_{\mathrm{k}}(\tau)=\theta_{\mathrm{k}}\left[\mathrm{J}(\mathrm{t})-\mathrm{J}_{\mathrm{g}}-\mathrm{t} / \eta\right]_{\mathrm{t}=\tau}=\left.\theta_{\mathrm{k}}[\mathrm{J}(\mathrm{t})-\mathrm{t} / \eta]\right|_{\mathrm{t}=\tau}
$$

where $\theta_{\mathrm{k}}$ is given by Eq. (58). It is easily seen that

$$
\theta_{\mathrm{k}}[1-\exp -\theta / \mathrm{T}]=-\theta_{\mathrm{k}}[\exp -\theta / \tau]
$$

and that therefore the approximations to the retardation spectrum from the creep compliance are (except for a change in sign) identical with the approximations to the relaxation spectrum from the relaxation modulus and have the same intensity functions.

With the previous change of variable, $\tau=1 / \zeta$, Eq. (77) can also be brought into the form of the Laplace transform, Eq. (73), where we now have

$$
\begin{gathered}
f(t)=J(t)-t / \eta-J e \\
\varphi(\zeta)=\left.T L(\tau)\right|_{\tau}=1 / \zeta
\end{gathered}
$$

To effect the transformation, we have made use of the relation 


$$
\int_{0}^{\infty} L(\tau) d \ln \tau=J_{e}-J_{g}
$$

where $J_{e}$ is the equilibrium compliance. Equation (76) then yields the same approximations which are obtained for the relaxation modulus except for a change in sign.

\section{Approximations from Other Viscoelastic Functions}

Other viscoelastic functions than those dealt with so far are used occasionally. The more important ones are creep recovery, stress relaxation after cessation of steady state flow, and deformation at constant rate of strain.

The creep recovery curve is

$$
J_{r e c}(t)=\int_{0}^{\infty} L(\tau)\left[1-\exp -t_{1} / \tau\right] \exp -t / \tau \cdot d \ln \tau+\left\{t_{1} / \eta\right\}
$$

where $t_{1}$ is the time at which creep recovery begins. Proceeding as in Section 5, the kth approximation to the retardation spectrum becomes

$$
L_{k}(\nu \tau)=\left.\frac{1}{1-\exp -t_{1} / \tau} \theta_{k}\left[J_{r e c}(t)\right]\right|_{t=\tau}
$$

where $\nu$ is the numerical factor by which the time scale of the spectrum must be adjusted when applicable.

For stress relaxation after cessation of steady-state flow we have

$$
\sigma_{\operatorname{cess}}(t)=R \int_{0}^{\infty} \tau H(\tau) \exp -t / \tau \cdot d \ln \tau
$$

where $R$ is the rate of strain. We now have

$$
\text { VI-24 }
$$


$\mathrm{H}_{\mathrm{k}}(\nu \tau)=\frac{1}{\mathrm{R} \tau} \theta_{\mathrm{k}}\left[\sigma_{\operatorname{cess}}(\mathrm{t})\right]_{\mathrm{t}=\mathrm{T}}$

For deformation at constant rate of strain

$$
\sigma_{R}(t)=\left\{G_{e} R t\right\}+R \int_{0}^{\infty} T H(\tau)[1-\exp -t / \tau] d \ell n \tau
$$

and consequently

$$
H_{k}(\nu \tau)=-\left.\frac{1}{R \tau} \theta_{k}\left[\sigma_{R}(t)-\left\{G_{e} R t\right\}\right]\right|_{t=\tau}
$$

Eqs. (86) and (88) are, of course, valid only if the behavior remains linearly viscoelastic throughout the deformation.

\section{Choice of Approximation Method (with D. G. Fesko)}

It was shown in the previous sections that if the order of the highest derivative is $k$ then there exist $k$ approximations to the spectra from the step and the storage functions and $k+1$ approximations from the los s functions. The question naturally arises which of the $k$ possible approximations one should use. The answer is relatively easy in case of the step functions and the special functions discussed in the previous section. All $\mathrm{k}$ approximations of a given order will have associated intensity functions whose maxima lie to the right of $\ln \theta / \tau=0$. The farther right the maximum the broader the intensity function (cf. Figs. 2 and 3), and the smaller the contribution to the approximation of the derivative of the highest order. The latter may be of practical advantage since it is difficult to obtain the 
the higher derivatives accurately and the higher the derivative the less well it will be determined. The broadness of the intensity function will be less important the smoother the spectrum. Generally one trades broadness of the intensity function against smaller contributions of the derivatives of higher order and this determines the choice.

The problem is a little more difficult in case of the dynamic functions. Two questions are involved:

(1) Where available, are the approximations with intensity functions whose maximum lies at $\ln \theta / \tau=0$ and which therefore do not require adjustment of the time scale of the spectrum, preferable over the others?

(2) In case one either has to, or wishes to select an approximation whose intensity function does not peak at the origin: is an approximation with intensity function maximum to the left of the origin preferable to one with the maximum to the right?

The situation may be clarified by considering Fig. 4. This contains a plot of the exact spectrum calculated by the Stieltjes transform inversion method ${ }^{(1)}$ from an analytic function fitted to the storage modulus data on polyisobutylene obtained by Fitzgerald, Grandine, and Ferry ${ }^{(6)}$. Also shown is a qualitative graphical representation of $I^{\prime}{ }_{2}(\theta / \tau)$ and of $I_{z_{R}}^{\prime}(\theta / \tau)$ at $T=\tau_{0}$. (The skewness of $I^{\prime}{ }_{z}{ }_{R}(\theta / \tau)$ is exaggerated). When $H(\theta) \cdot I^{\prime}{ }_{a}(\theta / \tau)$ is integrated from $\theta=0$ to $\theta=\infty$ for the parameter $\tau=\tau_{0}$, parts of the spectrum adjacent to $T_{0}$ contribute to the integral. If the skew is to the right as pictured in Fig. 4, H(T) to the right of $\tau_{0}$ will be weighted more heavily than that to the left of $\tau_{0}$. But $H(\theta)$ is a rapidly decreasing function at this point and the product $H(\theta) \cdot I_{2}^{\prime}(\theta / \tau)$ may approach zero as rapidly on the right side as on the left. 


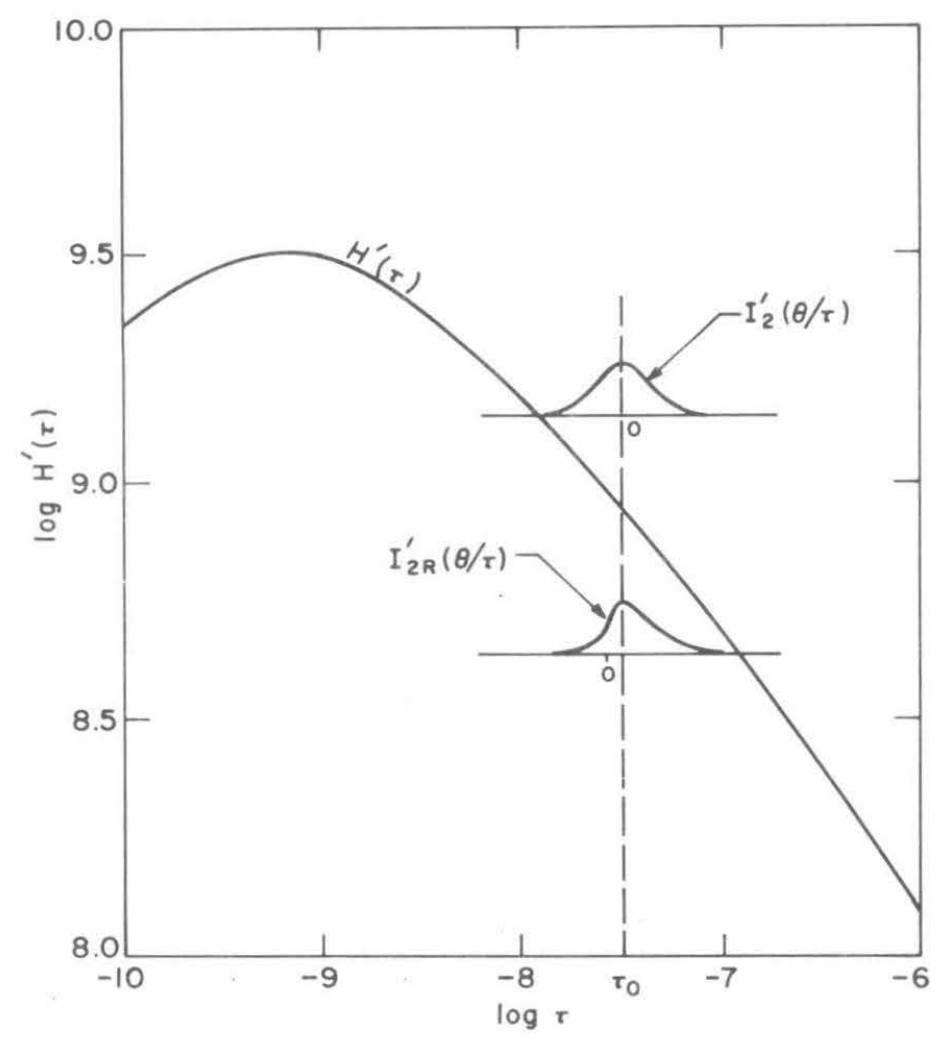

Fig. 4. Dependence of the Approximation on the Slope of the Spectrum.

Similarly, we would expect $I^{\prime}{ }_{2}(\theta / \tau)$, i. e. the intensity function skewed to the left, to provide a poor approximation resulting in an integral which would be too high. Deriving the spectrum at $\tau_{0}$ from $G^{\prime}(w)$ with a left skewed intensity function, therefore, would require a shift of greater magnitude than that indicated by inspection of the intensity function alone.

By the same argument, one would expect the symmetric intensity function, $I^{\prime}{ }_{a}(\theta / \tau)$, also to give approximations that are too high in any area where the spectrum is changing rapidly. Of course the symmetric case would provide a better overall approximation for both positive and negative slopes of the spectrum than the skewed case. 
The accuracy of the approximation (or the shift in $\tau_{0}$ required) is not dependent directly on the slope of $H(\tau)$ vs $\log \tau$, however. The integral is over the product of the intensity function and $\mathrm{H}(\mathrm{T})$; and therefore one is interested in the relative change in $\mathrm{H}(\tau)$ as one moves away from $\log \tau_{0}$. Thus we can say that the necessary shift will be dependent on the slope of $\log H(\tau)$ vs $\log \tau$ evaluated at $\log \tau_{0}$.

Using the polyisobutylene data tabulated in Table III of the previous report ${ }^{(1)}$, the second order approximations for the spectrum were evaluated along with the exact spectrum.

In Fig. $5 \log \mathrm{H}^{\prime} z_{\mathrm{R}}(\tau)$ and $\log \mathrm{H}^{\prime}{ }_{2}{ }_{\mathrm{L}}(\tau)$ are plotted with $\log \mathrm{H}(\tau)$, i. e. the exact spectrum. We see that $H^{\prime}{ }_{a_{R}}(\sqrt{2} T)$ works very well where

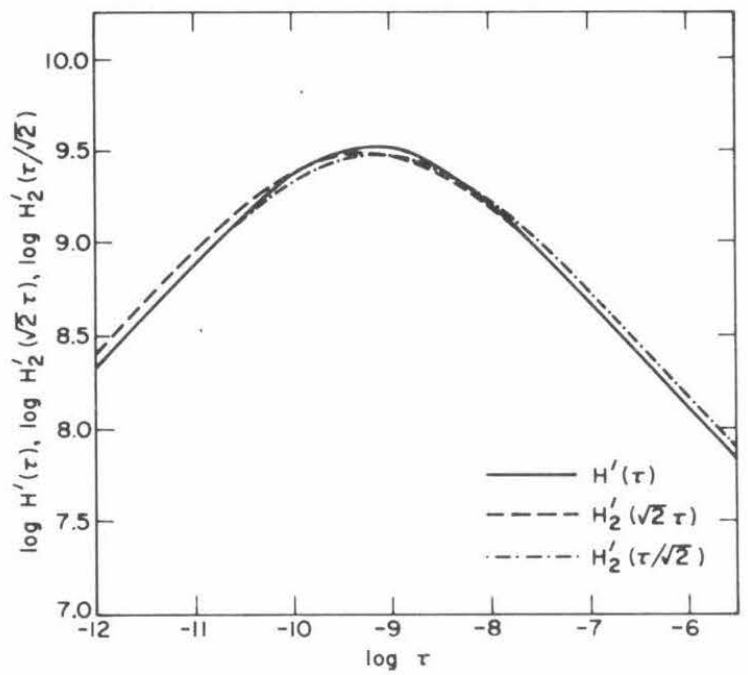

Fig. 5. $\log H^{\prime}(\tau), \log H_{2}^{\prime}(\sqrt{2} \tau)$, and $\log H_{2}^{\prime}(\tau / \sqrt{2})$ as Functions of $\log \tau$.

the slope of $\log H(\tau)$ is negative and $H^{\prime}{ }_{2}{ }_{L}(\tau / \sqrt{2})$ is most appropriate for a positive slope. The fine accuracy of the approximations in certain areas is partly coincidental, however, since a steeper or shallower slope of the log of the spectrum would shift the approximation above or below the exact 
values respectively. This becomes well apparent near the peak of the spectrum. Of course, at the peak, any approximation will give a low value because of the low order of any approximation of practical usefulness. In conclusion it may be said that in the calculation of the special functions from dynamic modulus data the asymmetric functions would probably be preferable to the symmetric ones; and that the right- or leftskewed functions should be used depending on the slope of the spectrum. An iterative procedure could be devised in which the shift factor could be modified according to need. 


\section{REFERENCES}

1. N. W. Tschoegl, "A Research Program on Solid Propellant Physical Behavior," MATSCIT PS 67-1, California Institute of Technology, Pasadena, California (1967), AFRPL-TR-67-193, Vol. I.

2. H. Fujita, J. Appl. Phys., 29:943 (1958).

3. R. D. Andrews, Ind. Eng. Chem., 44:707 (1953).

4. F. R. Schwarzl and A. J. Staverman, Appl. Sci. Res., Section A, $\underline{4}: 127$ (1953).

5. D. V. Widder, The Laplace Transform, Princeton University Press, Princeton, N. J., (1946), p. 277.

6. E. R. Fitzgerald, C. E. Grandine and J. D. Ferry, J. Appl. Phys., $\underline{24}: 650$ (1953). 

PART VII

THE APPLICATION OF THE Z-FORM NUMERICAL METHOD OF INVERSE LAPLACE TRANSFORMATION

TO PROBLEMS IN LINEAR VISCOELASTICITY

\author{
D. G. Fesko
}




\author{
PART VII \\ THE APPLICATION OF THE Z-FORM NUMERICAL \\ METHOD OF INVERSE LAPLACE TRANSFORMATION \\ TO PROBLEMS IN LINEAR VISCOELASTICITY
}

CONTENTS

1. Introduction. . . . . . . . . . . . . . . . . VII - 1

2. Interconversion of Viscoelastic Functions . . . . . VII - 1

3. The z-Form Method. . . . . . . . . . . . VII - 3

4. Applying the z-Form Method to Viscoelastic Problems . VII - 4 References . . . . . . . . . . . . . . . . VII - 14 


\section{ILLUSTRATIONS}

Fig. 1. Pole at $\mathrm{s}=0$. . . . . . . . . . . . . . VII - 5 
TABLES

Table I $D(t)$ Using z-Form Analysis . . . . . . . . . VII - 12 


\section{Introduction}

It is shown in the phenomenological theory of linear viscoelasticity that the transient viscoelastic functions, the creep compliance, and the relaxation modulus, are simply interconvertible after making the Laplace transformation. Mathematical techniques of finding the inverse Laplace transform therefore are of interest to both rheologists and stress analysts concerned with viscoelastic materials. Interconversion problems naturally arise in the design of solid propellant rocket motors.

This report first discusses difficulties encountered in the inversion of the Laplace transform of the transient viscoelastic functions when these are expressed as a Prony series. A potentially useful inversion technique, the $\mathrm{z}$-form method which has lately come into prominence in the field of control systems, is then outlined briefly, and finally its applicability to viscoelastic inversion problems is examined. The $\mathrm{z}$-form method is found to fail for viscoelastic functions which ordinarily represent material properties over a large time regime. The presence of short relaxation or retardation times in the spectral functions causes inherent instability when the method is applied to the long-time region of a viscoelastic function.

\section{Interconversion of Viscoelastic Functions}

To interconvert the transient viscoelastic functions through their Laplace transforms, it is necessary to cast them into a transformable analytic expression. This is conveniently done in form of a Prony or Dirichlet series ${ }^{(1)}$ which follows directly from the well-known representation of the transient functions by a series of Voigt or Maxwell elements connected in series or in parallel. In principle, the series should contain 
a very large number of terms. In practice the experimental curves are fitted by a small number, ordinarily of the order of 10 . Thus, if we let the number of terms be $n$, the creep compliance, $D(t)$, is given by

$$
D(t)=\sum_{i=1}^{n} D_{i}\left[1-\exp \left(-t / \tau_{i}\right)\right]
$$

and its Laplace transform becomes

$$
\bar{D}(s)=\sum_{i=1}^{n} \frac{D_{i}}{s\left(1+\tau_{i} s\right)}
$$

where $D_{i}$ and $\tau_{i}$ are constants (compliance and retardation time) for a parallel combination of spring and dashpot, and $s$ is the transform variable.

Similarly, the relaxation modulus $E(t)$ can be represented as

$$
E(t)=\sum_{i=1}^{n} E_{i} \exp \left(-t / \tau_{i}\right)
$$

with

$$
\bar{E}(s)=\sum_{i=1}^{n} \frac{E_{i} \tau_{i}}{1+\tau_{i} s}
$$

as the Laplace transform. $E_{i}$ and $T_{i}$ are the constants (modulus and relaxation time) for a series combination of spring and dashpot. The two functions can be simply related through their Laplace transforms. For example, the transform of the creep compliance $\bar{D}(\mathrm{~s})$ can be obtained from $\bar{E}(\mathrm{~s})$ by the relation 


$$
\bar{D}(s)=\frac{1}{s^{2} \bar{E}(s)}
$$

The constants, $D_{i}$ and $\tau_{i}$, or $E_{i}$ and $\tau_{i}$, may be found by the collocation method of Schapery ${ }^{(2)}$. Difficulties arise in this curve fitting procedure; for, if the retardation or relaxation times (the $\tau_{i}{ }^{\prime} s$ ) are too close together, the other parameters $\left(D_{i}\right.$ or $\left.E_{i}\right)$ may take on negative values which have no physical significance and lead to instability in the interconversion. Apart from this, however, use of Eq. (5) requires finding the roots of a polynomial of high degree with the attendant drawbacks. These difficulties can, in principle, be avoided by the use of the $\mathrm{z}$-form method which will now be outlined briefly.

\section{The z-Form Method}

A technique for numerically making the inverse Laplace transformation has been presented by Boxer and Thaler ${ }^{(3)}$ and further discussed by Gibson ${ }^{(4)}$. The inverse Laplace integral is first approximated by a finite integral

$$
f(t)=\frac{1}{2 \pi i} \int_{-i \infty}^{i \infty} f(s) \exp (s t) d s \cong \frac{1}{2 \pi i} \int_{-i \pi / T}^{i \pi / T} f(s) \exp (s t) d s
$$

Letting $\mathrm{t}=\mathrm{nT}$ and substituting $\mathrm{z}=\exp \mathrm{Ts}$,

$$
f(n T)=\frac{1}{2 \pi i} \oint \frac{1}{T} f(\ln z / T) z^{n-1} d z
$$

The contour in the s-plane transforms into a unit circle in the $\mathrm{z}$-plane. Since the contour is closed, the integral can be found by the residue 
theorem. First, however, an approximation is made for $\ln z$ which gives rise to the z-forms tabulated by Gibson ${ }^{(4)}$. For reasons of convergence, the $z$-forms are substituted for negative powers of $s$ in the Laplace transform, $f(s)$, and the resulting expression, $F(z)$, is arranged as a fraction with polynomials of $\mathrm{z}^{-1}$ forming the numerator and denominator. Instead of computing the residues directly, however, the fraction is first expanded in ascending powers of $\mathrm{z}^{-1}$. The coefficients of these terms automatically become $f(n T)$, the constant term giving $f(0)$; the coefficient of $z^{-1}, f(T)$; the coefficient of $z^{-2}, f(2 T)$; and so on. Barring computational round-off errors, this method becomes more accurate with smaller time increments, T. The technique is well suited to computer computations.

\section{Applying the $z-$ Form Method to Viscoelastic Problems}

The viscoelastic functions characterizing propellant behavior typically cover about twenty logarithmic decades ${ }^{(5)}$. The use of a single time increment in Eq. (7) is therefore out of the question. To cover the entire range, several time increments must be chosen. No problem arises if the selected time increment $T$ is much smaller than $\tau_{i}$. On the other hand, one can derive interesting results for the case in which $\mathrm{T}$ is much greater than $\tau_{i}$. To understand this behavior it is necessary to examine the various approximations that are made in the $\mathrm{z}$-form technique.

First, consider the effect of truncating the inverse Laplace integral at $\pm i \pi / T$ for the cases of a single negative real pole and for a single pole at zero. For a negative pole,

$$
f(t)=\mathcal{L}^{-1}\{f(s)\} \cong \frac{1}{2 \pi i} \int_{-\pi / T}^{i \pi / T} \frac{\exp (s t)}{s+a} d s
$$


Making the substitution $y=s+a$, we obtain

$$
f(t)=\frac{\exp (-a t)}{2 \pi i} \int_{-a-i \pi / T}^{-a+i \pi / T} \frac{\exp (y t)}{y} d y
$$

This integral can be represented by an infinite series

$$
f(t)=\frac{\exp (-a t)}{2 \pi}\left[\log y+y t+\frac{(y t)^{2}}{2 \cdot 2 !}+\frac{(y t)^{3}}{3 \cdot 3 !} \cdots\right]_{y=-a-i \pi / T}^{y=-a+i \pi / T}
$$

The series converges faster than an exponential, and no unstable behavior results as one would expect $f(t)$ to vanish as $T$ becomes large, $(t=n T)$. Treating the pole at zero is a little more difficult. It can be considered by deforming the usual contour $A$ into the semicircle shown as contour B in Fig. 1 .

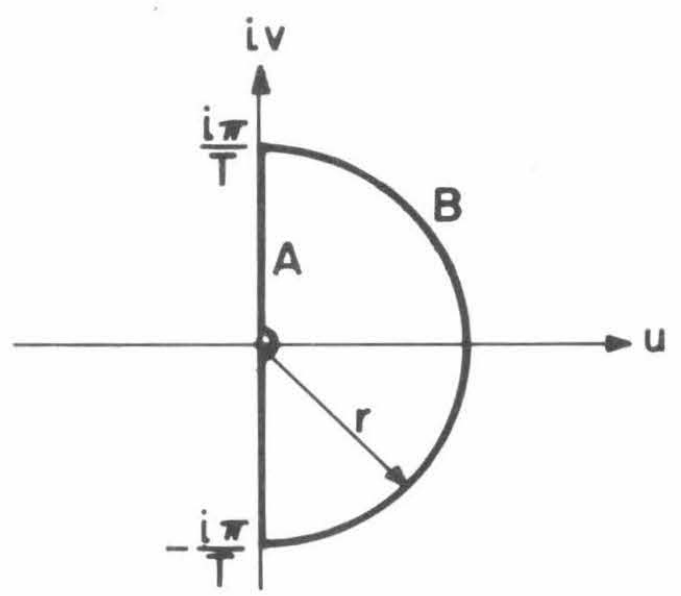

Fig. 1. Pole at $\mathrm{s}=0$.

The integral over contour A equals the integral over contour B. Letting $\mathbf{s}=r \exp (i \theta)$, where $r=\pi / T$, 


$$
\begin{aligned}
f(t)=f(n T) & \cong \frac{1}{2 \pi i} \int_{-\pi / 2}^{\pi / 2} \frac{\exp \left(n T r e^{i \theta}\right) i r \exp (i \theta)}{r \exp (i \theta)} d \theta \\
& \cong \frac{1}{2 \pi} \int_{-\pi / 2}^{\pi / 2} \exp \left(n \pi e^{i \theta}\right) d \theta
\end{aligned}
$$

Taking absolute values to place a bound on the error, we have

$$
\left|\frac{1}{2 \pi} \int_{-\pi / 2}^{\pi / 2} \exp [n \pi(\cos \theta+i \sin \theta)] d \theta\right| \leqq \frac{1}{\pi} \exp (n \pi \cos \theta) d \theta
$$

The integral on the right hand side is always smaller than

$$
\frac{1}{\pi} \int_{0}^{1} \exp \left[n \pi\left(1-x^{2}\right)\right] d x=\operatorname{erf}(1) \frac{\exp (n)}{\pi \sqrt{n \pi}}
$$

Thus, we might expect a pole at zero to give rise to behavior slightly better than an exponential of $n$, but it would still cause divergence if $n$ increases without bound.

To examine now the effect of substituting $z$-forms for the reciprocal of the Laplace variable let us treat three individual cases first.

Case 1. Single pole at zero: $f(s)=s^{-1}$ From Gibson

$$
\left.F(z)=\frac{T}{2}\left(1+z^{-1}\right) / 1-z^{-1}\right)
$$


Then

$$
\begin{aligned}
f(n T) & =\frac{1}{2 \pi i} \oint \frac{1}{2}\left[\left(1+z^{-1}\right) z^{n-1} /\left(1-z^{-1}\right)\right] d z \\
& =\frac{1}{2} \text { Residue }\left\{z^{n-1} /(z-1)\right\}_{z=1}=1
\end{aligned}
$$

The function transforms correctly and is independent of $\mathrm{T}$.

Case 2. Single negative pole: $f(s)=(a s+1)^{-1}$

$$
F(z)=\frac{T}{2}\left(1+z^{-1}\right) /\left[\left(1-z^{-1}\right) a+\frac{T}{2}\left(1+z^{-1}\right)\right] \approx 1 \text { as } T \rightarrow \infty
$$

Then

$$
\lim _{T \rightarrow \infty} f(n T) \cong \lim _{T \rightarrow \infty} \frac{1}{2 \pi i} \oint z^{n-1} / T d z=0
$$

The response vanishes as $T$ becomes large indicating stable behavior.

Case 3. Single negative pole and pole at zero: $f(s)=\left(a s^{2}+s\right)^{-1}$

$$
F(z)=\frac{T^{2}}{12}\left(1+10 z^{-1}+z^{-2}\right) /\left[a\left(1-z^{-1}\right)^{2}+\frac{T}{2}\left(1+z^{-1}\right)\left(1-z^{-1}\right)\right]
$$

Evaluating residues,

$$
\lim _{T \rightarrow \infty} f(n T) \cong 1+\frac{2}{3}(-1)^{n-1}
$$

The response shows oscillatory behavior but no growth.

These three cases treat simple $z$-form approximations to the Laplace inversion when the time increment used is large. Instead of 
expanding $F(z)$, however, its poles are found so that a closed form of the solution can be evaluated from the residues. Case 1 treats a single pole in the $s-p l a n e$ at $s=0$. This is a unit step function in time and is seen to transform well. Case 2 treats a single negative, real pole and the contribution of this term is seen to vanish as $1 / \mathrm{T}$ as $\mathrm{T}$ becomes large. This is not as fast as the exponential decay that should appear, but it would probably be sufficient for most applications. In Case 3, which represents the combination of Cases 1 and 2 , one sees that the solution is oscillatory. Case 3 is also the transform of one of the terms in the sum in Eq. (1). Therefore, unless the $\mathrm{D}_{i}{ }^{\prime}$ s change by at least one order of magnitude with each term, which does not usually happen, one would expect a significant oscillatory error in the inversion of the above single function.

The cases considered have been simple, but the $z$-form method should be most valuable when applied to more complicated fractions with polynomials of $\mathrm{s}$ in the numerator and denominator. In general, $\mathrm{z}$-forms are substituted for powers of $\left(\mathrm{s}^{-1}\right)$. For a term $\mathrm{x}^{-\mathrm{n}}$, the $\mathrm{z}$-form becomes

$$
\frac{T^{n}}{k_{n}} \frac{N_{n}\left(z^{-1}\right)}{\left(1-z^{-1}\right)^{n}}
$$

where $k_{n}$ is a constant and $N_{n}\left(z^{-1}\right)$ is a polynomial of $z^{-1}$ in which the highest order term is $z^{-n}$. Thus, if $f(s)$ is a single fraction with polynomials of $s^{-1}$ (or of $s$ ) in the numerator and denominator, and we let $\mathrm{T}$ be large, only the highest order terms (in $\mathrm{s}^{-1}$ ) will be important after substitution of the $z$-forms. We can therefore discuss two general cases, keeping only the highest order terms in $s^{-1}$. 
Case 4. General case:

$$
f(s)=\frac{1+\cdots \cdots+s^{m-1}}{1+\cdots \cdots+s^{m}} \cong \frac{s^{-m}}{s^{-m}}
$$

Then

$$
F(z) \cong 1
$$

and $f(n T)$ approaches zero as $1 / T$ as $T$ becomes large.

Case 5. General case with a pole at zero:

$$
f(s)=\frac{1+\cdots \cdots+s^{m-1}}{s+\cdots+s^{m}} \cong \frac{s^{-m}}{s^{-m+1}}
$$

Then

$$
F(z) \cong \frac{k_{m} T}{k_{m-1}} \frac{N_{m}\left(z^{-1}\right.}{\left(1-z^{-1}\right) N_{m-1}\left(z^{-1}\right)}
$$

and

$$
f(n T) \cong \oint \frac{N_{m}\left(z^{-1}\right) z^{n-1}}{\left(1-z^{-1}\right) N_{m-1}\left(z^{-1}\right)} d z
$$

neglecting the $\mathrm{k}_{i}{ }^{\prime} \mathrm{s}$ for simplicity. In the two cases shown above, $f(s)$ has a numerator which is a polynomial of $\mathrm{s}$ of smaller order than the denominator. This is true of almost any physical problem.

Case 4 includes Case 2 as a special case. Both numerator and denominator have a constant term so that clearing the fraction of positive powers of $s$ results in a fraction with the highest power of $s^{-1}$ in both the numerator and the denominator. In substituting the $z$-forms, the 
numerator and denominator tend to approach one another as $\mathrm{T}$ gets large. We are then left with the same situation as in Case 2 in which the integral approaches zero as $1 / T$. If the numerator lacks the constant term, one can see that the resulting $\mathrm{z}$-form approximation will go to zero even faster.

Case 5 includes Case 3 as a special case. The function $f(s)$ has a pole at $s=0$; in other words, the denominator of $f(s)$ lacks the constant term while the numerator still has it. As a result, $z$-form substitution yields a numerator with $\mathrm{T}$ raised to one power higher than the denominator. This $T$ cancels with the $1 / T$ in the integral (Eq. (7)) leaving no $T$ dependence. It is now necessary to consider the poles of $F(z)$. If any of the poles have real parts that are greater in absolute value than unity, the $\mathrm{z}^{\mathrm{n}-1}$ term in the integral will cause exponential growth. By examining the numerators of the first few $z$-forms given by Gibson ${ }^{(4)}$ one concludes that there will be poles of this type. In fact, the $z$-form for $s^{-1}$ and $s^{-3}$ are probably the only ones that do not cause exponential growth under these circumstances, and they impart an oscillatory nature to the result as in Case 3 . This can be demonstrated on the practical example presented by Boxer and Thaler ${ }^{(3)}$. For illustration, they provide a very well behaved function

$$
f(s)=\frac{1}{s+s^{2}+s^{3}}
$$

When the preceeding analysis is applied to this problem, the response becomes

$$
f(n T)=\frac{1}{6}\left[1-\frac{1}{2}(-5+2 \sqrt{6})^{n}-\frac{1}{2}(-5-2 \sqrt{6})^{n}\right]
$$

for large $T$. One can see that the third term in the expression grows 
exponentially in $n$ and that $f(n T)$ will change sign with each successive $n$. Theoretically, the $z$-form method should still work, since the contour for the integral shown in Eq. (7) is taken on the unit circle. The poles outside the contour should not be counted. In the numerical treatment however, everything is included, causing the approximation to "blow up."

Since the Laplace transform of the creep compliance is given by Eq. (2), the form of the combined fraction would be like that described in Case 5. One would expect the $z$-form technique to yield an unstable solution. Even if the technique were applied to the individual terms in the sum in Eq. (2), which results in oscillatory behavior (Case 3), practical values for the $\mathrm{D}_{i}{ }^{\prime} \mathrm{s}$ would probably not differ sufficiently that the contribution of the oscillatory terms $\left(\tau_{i}<T\right)$ would die out fast enough relative to the other terms $\left(\tau_{i}>\mathrm{T}\right)$.

Computer calculations have given unstable responses similar to the case for the Boxer and Thaler example. For a test, creep compliance data for polyisobutylene were taken from Ferry ${ }^{(6)}$, and treated by the collocation method of Schapery ${ }^{(2)}$ to arrive at a representation of the creep compliance curve in the form of Eq. (1) valid for $\log t$ from -14 to +2 . The Laplace transform is represented by the sum in Eq. (2). Transforming it term by term by the $\mathrm{z}$-form method leads to oscillations as predicted by Case 3 . If, on the other hand, all the terms are combined into a single fraction, transformation of the fraction by the $\mathrm{z}$-form method, and selecting various time increments ( $\mathrm{T})$, results in an even more pronounced instability. For instance, choosing $\mathrm{T}=1.526 \times 10^{-4} \mathrm{sec}$, one obtains the results shown in Table I. 
TABLE I

\section{$\underline{D(t)}$ Using z-Form Analysis}

$\begin{array}{cc}\left(\mathrm{T}=1.526 \times 10^{-4} \mathrm{sec}\right) & \underline{\mathrm{D}(\mathrm{t})} \\ 0 & 3.7 \times 10^{-2} \\ \mathrm{~T} & -5.4 \times 10^{-5} \\ 2 \mathrm{~T} & 1.3 \times 10^{-3} \\ 3 \mathrm{~T} & -2.8 \\ 4 \mathrm{~T} & 6.1 \times 10^{2} \\ 5 \mathrm{~T} & -1.3 \times 10^{5} \\ 6 \mathrm{~T} & 2.8 \times 10^{7} \\ 7 \mathrm{~T} & -6.2 \times 10^{9} \\ 8 \mathrm{~T} & 1.3 \times 10^{12} \\ 9 \mathrm{~T} & -2.8 \times 10^{14} \\ 10 \mathrm{~T} & 6.2 \times 10^{16}\end{array}$

The chosen time interval, $T$, is large compared with most of the $\tau_{i}{ }^{\prime} s$ which range from $10^{-14}$ to $10^{2}$ seconds.

The creep compliance, $D(t)$, was chosen for discussion because it appears to be more closely suited to Laplace transform inversion. It is simply represented and increases in magnitude with increasing time. By contrast the relaxation modulus, $E(t)$, is a decreasing function of time. Thus the first interval of the $z$-transform will not contain a jump at $t=0$ when the transformation is applied to the creep compliance. The instability of the z-transform method when the time increments are large, would show up similarly, however, if the method were applied to the relaxation 
modulus or to any other of the viscoelastic functions. It must therefore be concluded that the method is not suitable to the viscoelastic conversion problem when the data are supplied as a function of logarithmic time or frequency. 


\section{REFERENCES}

1. M. L. Williams, P. J. Blatz and R. A. Schapery, "Fundamental Studies Relating to Systems Analysis of Solid Propellants," GALCIT SM 61-5, California Institute of Technology, Pasadena, California, 1961.

2. R. A. Schapery, Proc. Fourth U. S. Nat. Congress Appl. Mech., Vol. 2, p. 1075, ASME, New York, 1962.

3. R. Boxer and S. Thaler, "A Simplified Method of Solving Linear and Nonlinear Systems," Proc. IRE, 44, 89 (1956).

4. J. E. Gibson, Nonlinear Automatic Control, pp. 147-159, McGrawHill, New York (1963).

5. N. W. Tschoegl and T. L. Smith, "Viscoelastic Properties of Solid Propellants and Propellant Binders, "Final Report, SRI Prospect PRU-3739, 4660, 5174, Stanford Research Institute, Menlo Park, California, 1966.

6. J. D. Ferry, Viscoelastic Properties of Polymers, John Wiley, New York, 1961, p. 77. 

PART VIII

RESPONSE OF VISCOELASTIC MATERIALS TO

PIECEWISE CONTINUOUS EXCITATIONS

\author{
K. Yagii \\ and
}

N. W. Tschoegl 
RESPONSE OF VISCOELASTIC MATERIALS TO

PIECEWISE CONTINUOUS EXCITATIONS

CONTENTS

1. Introduction. . . . . . . . . . . . . . . . VIII - 1

2. Piecewise Continuous Functions . . . . . . . . . VIII - 2

3. Staircase Function . . . . . . . . . . . . . VIII - 8

4. Pyramid Function. . . . . . . . . . . . . . VIII - 11

5. Triangular Pulse. . . . . . . . . . . . . . VIII -13

6. Triangular Pulse Train. . . . . . . . . . . . VIII -15

7. Other Piecewise Continuous Excitations . . . . . . VIII - 21

References . . . . . . . . . . . . . . . VIII -22 


\section{ILLUSTRATIONS}

Fig. 1. The Step Function . . . . . . . . . . . . VIII - 4

Fig. 2. Relaxation Modulus and Creep Compliance of Three-Parameter Maxwell Model . . . . . . VIII - 7

Fig. 3. Staircase and Pyramid Excitation and Response. . VIII - 9

Fig. 4. Triangular Single Pulse and Pulse Train Excitation and Response. . . . . . . . . . . . . . VIII - 14 


\section{TABLES}

Table I Laplace Transform Pairs Involving the Unit Step Function . . . . . . . . . . . . VIII - 5 


\section{Introduction}

In cumulative damage testing viscoelastic materials are subjected to piecewise continuous excitation functions (see, e.g. Ref. (1)), but few experiments have so far been made on the stress-strain behavior of viscoelastic materials under these conditions. Tanaka and Smith ${ }^{(2)}$ have examined the creep behavior of several rubbers under a pyramid excitation, in which the loads were applied (and subsequently removed) in approximately equal increments at fixed intervals of time.

It is of interest to be able to predict the behavior of a given material under a piecewise continuous periodic excitation when the behavior under a standard excitation such as a step-function of stress or strain (i.e. the stress relaxation or the creep curve) is known. An attempt can then be made to ascribe departures from the predicted behavior to cumulative damage. In this way it might be possible to predict fatigue failure in a non-destructive test from observation of the material during the early stages of the loading-unloading cycles.

The first step in this direction is the development of the formal mathematical apparatus for the description of the response of viscoelastic materials to piecewise continuous excitations on the basis of linear theory. The required methods are well known in electric circuit theory but are less familiar to most polymer rheologists. This report outlines a general method for the formulation of the response of linear viscoelastic materials to piecewise continuous excitations in terms of the standard response functions (i.e. the stress relaxation modulus or creep compliance, and, additionally in the case of periodic excitations, the real and imaginary parts of the complex modulus or compliance). The application of the 
general method is demonstrated on the most common piecewise continuous excitations, the
1) staircase
2) pyramid
3) triangular pulse, and
4) triangular pulse train excitation.

\section{Piecewise Continuous Functions}

A function $f(t)$ is piecewise, or sectionally continuous on a finite interval $\mathrm{a} \leq \mathrm{t} \leq \mathrm{b}$ if it is possible to subdivide that interval into a finite number of subintervals in each of which $f(t)$ is continuous and has finite limits as $t$ approaches either end point of the subinterval from the interior ${ }^{(3)}$. Any discontinuties of such a function in the interval $(a, b)$ are of the type known as ordinary points of discontinuity where the values of the function makes a finite jump (3). This class of functions includes, of course, continuous functions.

In the mathematical representation of piecewise continuous functions the unit step function plays a basic role.

The unit step-function, $h(t)$, is defined by

$$
h(t)= \begin{cases}0 & t<0 \\ 1 & t>0\end{cases}
$$

i. e. the function has the value 0 for times smaller than 0 and the value 1 for times larger than 0 . The physical interpretation of the step-function is that of a constant excitation imposed at the instant $t=0$. If the excitation is imposed at $\mathrm{t}=\mathrm{t}^{\prime}$ instead of $\mathrm{t}=0$, then 


$$
h\left(t-t^{\prime}\right)= \begin{cases}0 & t<t^{\prime} \\ 1 & t>t^{\prime}\end{cases}
$$

The two functions are shown in Figs. la and $1 \mathrm{~b}$.

Equation (la) can be used to restrict a given function $\varphi(t)$ to its values for $t>0$, as shown in Fig. lc, where

$$
f(t)=\varphi(t) h(t)
$$

is plotted as a function of $t$. Multiplication of $\varphi(t)$ by $h(t)$ thus results in a new function, $f(t)$, in which that portion of $\varphi(t)$ for which $t<0$ is cut off. Equation (ld)

$$
f(t)=\varphi(t) h\left(t-t^{\prime}\right)
$$

serves in a similar way to cut off $\varphi(t)$ for values of $t<t^{\prime}$ as shown in Fig. ld. Translation of $\varphi(t) h(t)$ along the $t$ axis by the amount ${ }^{\prime}$ is achieved by writing

$$
f(t)=\varphi\left(t-t^{\prime}\right) h\left(t-t^{\prime}\right)
$$

This function is shown in Fig. le. To cut off the function for values of $t>t^{\prime \prime}$ one writes

$$
f(t)=\varphi\left(t-t^{\prime}\right) h\left(t-t^{\prime}\right)-\varphi\left(t-t^{\prime \prime}\right) h\left(t-t^{\prime \prime}\right)
$$

and this is illustrated in Fig. 1f. The second term on the right annihilates the effect of the first term when $t>t$ ". In Eqs. (lc) through (lf) $\varphi(t)$ is a 


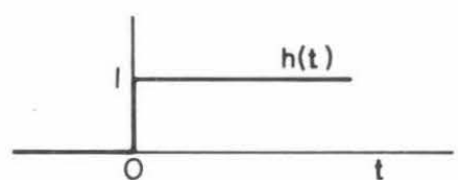

(a)

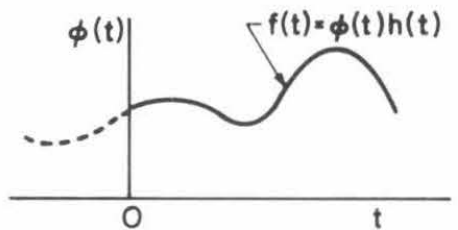

(c)

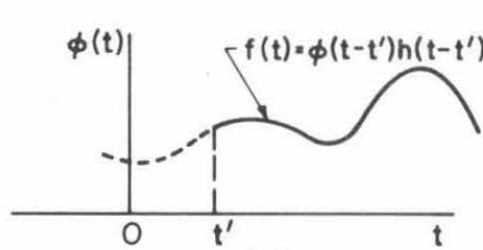

(e)

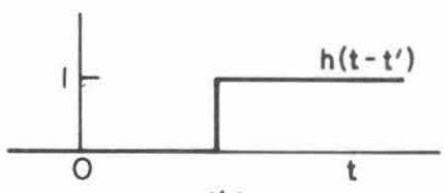

(b)

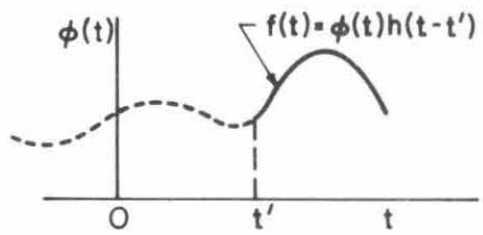

(d)

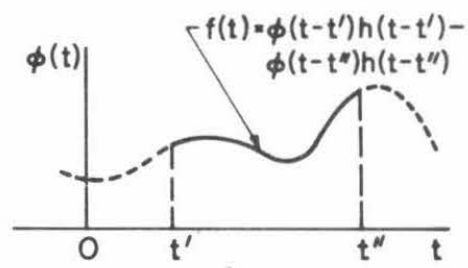

(f)

Fig. 1. The Step Function.

continuous function defined on the interval $-\infty<t<\infty$. The function $f(t)$ on the other hand is non-zero only on the interval $0<t<\infty$ in Eq. (lc), on the interval $\mathrm{t}^{\prime}<\mathrm{t}<\infty$ in Eqs. (ld) and (le), and on the interval $\mathrm{t}^{\prime}<\mathrm{t}<\mathrm{t}^{\prime \prime}$ in Eq. (lf).

Piecewise continuous excitations typically consist of a series of sectionally smooth functions defined in an interval ( $\left.t^{\prime}, t^{\prime \prime}\right)$ such as $f(t)$ in Fig. If. If the same function is repeated, the excitation is called periodic. The mathematical treatment of piecewise continuous functions is greatly simplified by the Laplace transformation since the expressions will generally be of closed form in the transform plane. Table I lists some Laplace transform pairs involving the unit step-function which will be needed in this report. 
TABLE I

\section{Laplace Transform Pairs Involving the Unit Step Function}

No.

1

2

3

4

5

6

7

8

$$
\left[\int_{0}^{t-n t^{\prime}} f(u) d u\right] h\left(t-n t^{\prime}\right)
$$

$f(t)$

$$
f(t) h(t)
$$$$
f\left(t-t^{\prime}\right) h\left(t-t^{\prime}\right)
$$$$
\mathrm{h}(\mathrm{t})
$$$$
\mathrm{h}\left(\mathrm{t}-\mathrm{nt} \mathrm{t}^{\prime}\right)
$$

$$
\left(t-n t^{\prime}\right) h\left(t-n t^{\prime}\right)
$$

$$
\left[\exp -\left(t-n t^{\prime}\right) / \tau\right] h\left(t-n t^{\prime}\right)
$$

$$
\left[1-\exp -\left(t-n t^{\prime}\right) / \tau\right] h\left(t-n t^{\prime}\right)
$$

$$
\frac{\exp -n t^{\prime} s}{s(1+\tau s)}
$$

To derive Transform Pairs (3) - (8) use has been made of the Translation Theorem expressed compactly in the form

$$
f\left(t-t^{\prime}\right) h\left(t-t^{\prime}\right)=\mathcal{L}^{-1}\left[\bar{f}(s) \exp -t^{\prime} s\right]
$$

where $\mathcal{L}^{-1}$ denotes the operation of taking the inverse of the Laplace transform. In words: If the function $f(t)$ is translated along the t-axis by the amount $t^{\prime}$, the transform $\bar{f}(s)$ is multiplied by the exponential factor exp-t's. The Translation Theorem is included as Pair (2) in Table I. 
A powerful device for obtaining the transform of periodic functions is the Periodicity Theorem which may be stated compactly as

$$
\bar{f}(s)=\frac{\bar{f}_{t} /(s)}{1-\exp -t^{\prime} s}
$$

or in words: The transform $\bar{f}(s)$ of a periodic function is obtained by dividing the transform $\bar{f}_{t}(s)$ of the "repeat" function, defined in the interval $0<\mathrm{t}<\mathrm{t}^{\prime}$, by $1-\exp -\mathrm{t}^{\prime} \mathrm{s}$. Thus it is only necessary to derive the transform of $f_{t}{ }^{\prime}(t)$ to obtain $f(t)=f\left(t+n t^{\prime}\right)$.

We are now ready to derive the relation between the response of linearly viscoelastic materials to piecewise continuous and standard excitations. The relations will be developed for the general case where $\&(t), R(t)$, and $m(t)$ are the time-dependent excitation, response, and material function, respectively. These relations can easily be specialized to stress-strain and creep compliance, or strain-stress and relaxation modulus. The resulting expressions can either be evaluated numerically, or the standard response function may be fitted to a suitable analytic expression. The collocation method of Schapery ${ }^{(5)}$ might be useful for this purpose.

To illustrate representative behavior, the response to the piecewise continuous excitation functions listed in Section 1 were calculated using, as the characteristic material function, the shear relaxation modulus, $G(t)$, and the shear creep compliance, $J(t)$, derived from the three-parameter Maxwell model shown in Fig. 2. The relaxation modulus is obtained from

$$
G(t)=G_{1}+G_{2} \exp -t / T
$$




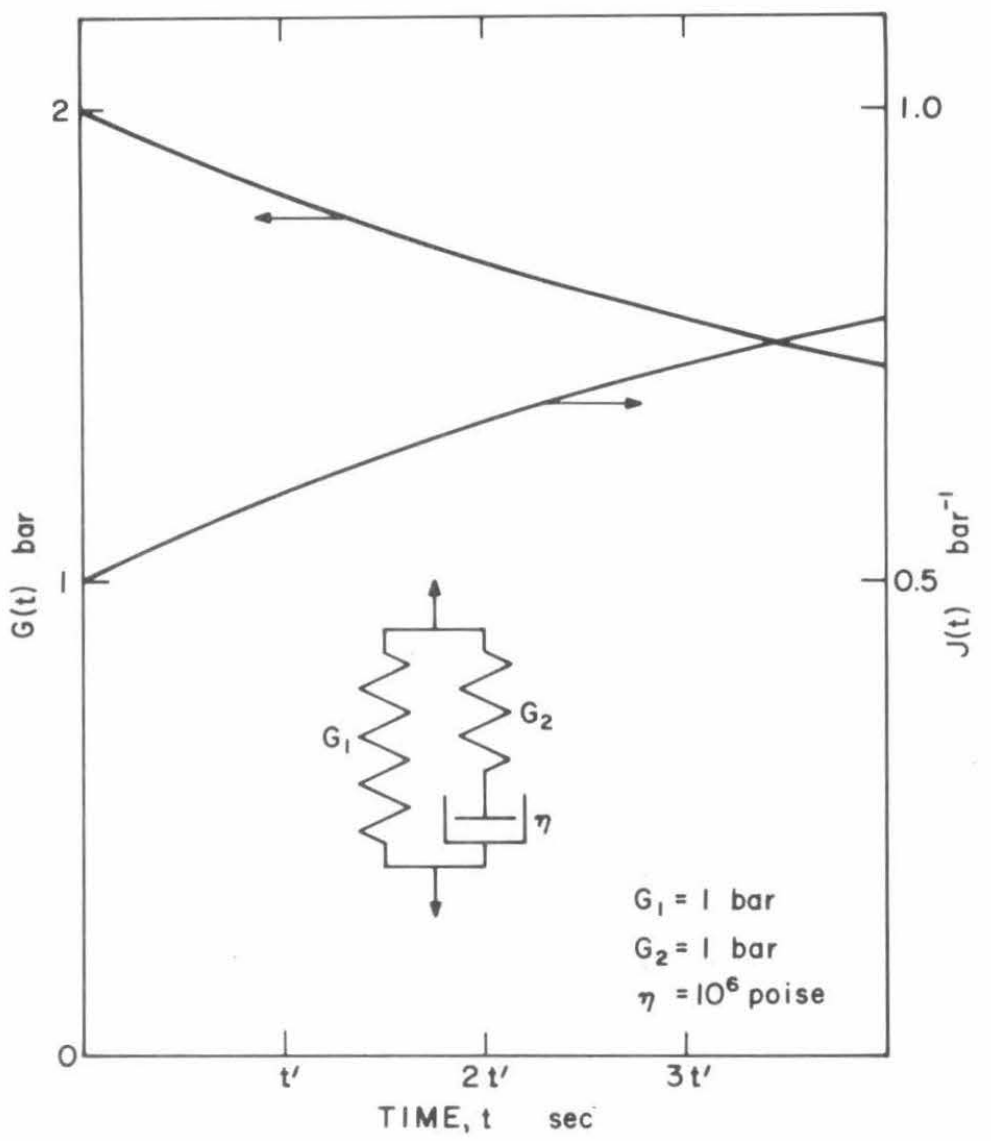

Fig. 2. Relaxation Modulus and Creep Compliance of Three-Parameter Maxwell Model.

and the creep compliance from

$$
J(t)=J_{1}\left[1-\frac{J_{1}}{J_{1}+J_{2}} \exp -t / \lambda\right]
$$

where $T=\eta / G$ is the relaxation time, and $\lambda=\left(J_{1}+J_{2}\right) \eta$ is the retardation time. The numerical values of $G_{1}=1 / J_{1}, G_{2}=1 / J_{2}$, and $\eta$ were 1 bar $\left(10^{6}\right.$ dyne $\left./ \mathrm{cm}^{2}\right)$, and $10^{6}$ poise, respectively. 


\section{Staircase Function}

The general stair case excitation consists of a superposition of several step-functions, each of different height and duration. In the $\underline{\text { regular }}$ staircase excitation shown in the left half of Fig. 3 the step heights are all equal and so are the intervals. The excitation function is

$$
\&(t)=\sum_{n=0}^{N} e_{n} h\left(t-t_{n}\right)
$$

which transforms to

$$
\bar{\delta}(s)=\sum_{n=0}^{N} \frac{e_{n} \exp -t_{n} s}{s}
$$

by Transform Pair (4). For the regular function $e_{0}=e_{1}=\cdots=e_{N}$, and $t_{n}=n t^{\prime}$, where $t^{\prime}$ is the duration of the interval, and $e_{0}$ is the step height. Thus we have

$$
\bar{\delta}(s)=e_{0} \sum_{n=0}^{N} \frac{\exp -n t^{\prime} s}{s}
$$

Since the transform of the step function of height $e_{o}$ is $e_{o} / s$,

Eq. (7) could have been obtained by substituting

$$
\bar{f}_{t}(s)=\bar{\delta}_{t}(s)=\frac{e_{0}}{s}
$$

into Eq. (3). This gives 


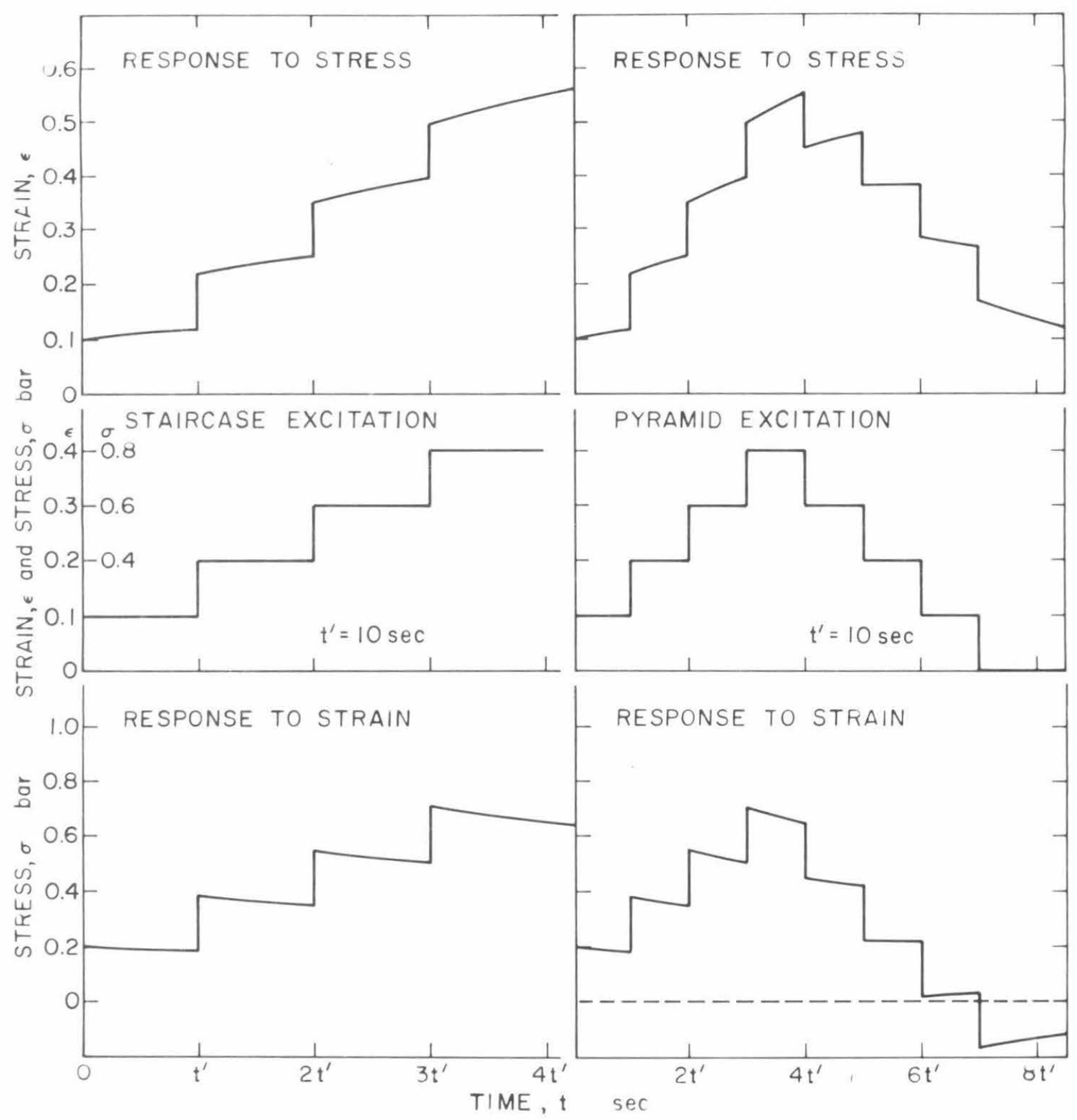

Fig. 3. Staircase and Pyramid Excitation and Response. 


$$
\bar{\delta}(s)=\frac{e_{\circ}}{s(1-\exp -t s)}
$$

which may be expanded and truncated to yield Eq. (7). Use of the Periodicity Theorem thus eliminates the need for formulating the excitation in terms of translated step-functions.

Since

$$
\bar{R}(s)=s \bar{m}(s) \bar{\delta}(s)
$$

we obtain the response transform as

$$
\bar{R}(\mathrm{~s})=\mathrm{e}_{\circ} \sum_{\mathrm{n}=0}^{\mathrm{N}} \bar{m}(\mathrm{~s}) \exp -\mathrm{nt} \text { 's }
$$

or

$$
R(t)=e_{0} \sum_{n=0}^{N} m\left(t-n t^{\prime}\right) h\left(t-n t^{\prime}\right)
$$

by Transform Pair (2).

Specializing, we obtain for a strain excitation

$$
\sigma(t)=e_{0} \sum_{n=0}^{N} G\left(t-n t^{\prime}\right) h\left(t-n t^{\prime}\right)
$$

and for a stress excitation

$$
\varepsilon(t)=\sigma_{0} \sum_{n=0}^{N} J\left(t-n t^{\prime}\right) h\left(t-n t^{\prime}\right)
$$


where $\sigma(\mathrm{t})$ and $\varepsilon(\mathrm{t})$ are the time-dependent stress and strain responses. The responses of the three parameter model, Fig. 2, to the staircase excitation are shown in the left half of Fig. 3. It should be noted that the rate of creep and the rate of stress relaxation increase as the stress or the strain increase in each successive interval $t^{\prime}$.

The results embodied in Eqs. (13) and (14) could, of course, have been obtained by substituting

$$
\delta(t)=e_{0} \sum_{n=0}^{N} h\left(t-n t^{\prime}\right)
$$

into the Boltzman superposition integral

$$
R(t)=\int_{0}^{t} m(t-u) \frac{d \&(u)}{d u} d u
$$

In cases which are more complicated than the elementary one which was chosen here for illustration, the Laplace transform method is generally more convenient.

\section{Pyramid Excitation}

The pyramid excitation shown in the center portion of the right half of Fig. 3 consists of the combination of an ascending and a descending staircase. For the regular pyramid function therefore

$$
\delta(t)=e_{0}\left[\sum_{n=0}^{M} h\left(t-n t^{\prime}\right)-\sum_{n=M+1}^{N} h\left(t-n t^{\prime}\right)\right]
$$


which transforms to

$$
\bar{\delta}(s)=e_{0}\left[\sum_{n=0}^{M} \bar{m}(s) \exp -n t^{\prime} s-\sum_{n=M+1}^{N} \bar{m}(s) \exp -n t^{\prime} s\right]
$$

The resulting response functions become

$$
\sigma(t)=e_{0}\left[\sum_{n=0}^{M} G\left(t-n t^{\prime}\right) h\left(t-n t^{\prime}\right)-\sum_{n=M+1}^{N} G\left(t-n t^{\prime}\right) h\left(t-n t^{\prime}\right)\right]
$$

and

$$
\varepsilon(t)=\sigma_{0}\left[\sum_{n=0}^{M} J\left(t-n t^{\prime}\right) h\left(t-n t^{\prime}\right)-\sum_{n=M+1}^{N} J\left(t-n t^{\prime}\right) h\left(t-n t^{\prime}\right)\right]
$$

The responses of the three-parameter model (with $M=3, N=7$ ) are shown in the top and bottom portion of the right side of Fig. 3. It should be noted that for this particular model the strain still increases after the first decrease in the stress at $t=4 t^{\prime}$ albeit at a reduced level, and is almost level after the following decrease. Only after the third decrease does the creep curve turn into a typical creep recovery curve. Although the stress is removed completely at $t=n t '$, the creep recovery continues for several intervals before the strain is finally reduced to zero. This is a clear demonstration of the viscoelastic "persistence of memory". A similar observation can be made on the response to the pyramidal strain excitation. Following the last decrease in the strain the stress actually reverses direction before it relaxes to zero several intervals later. 
The responses shown here are idealized linearly viscoelastic responses. In practice it is difficult to remain within the linear response region when the excitation is increased by several increments. The effect of large deformations on creep recovery has been discussed by Leaderman ${ }^{(6)}$. Tanaka and Smith ${ }^{(2)}$ also discussed the large deformations involved in their pyramidal creep recovery on the basis of the factorizability of the effects of strain and time.

\section{Triangular Pulse}

In a triangular pulse the excitation increases at a constant rate $\mathrm{R}^{\prime}=\mathrm{e}_{\mathrm{o}} / \mathrm{t}^{\prime}$ for $\mathrm{t}=0$ to $\mathrm{t}=\mathrm{t}^{\prime}$ and thereupon decreases at the rate $\mathrm{R}^{\prime \prime}=\mathrm{e}_{\mathrm{o}} / \mathrm{t}^{\prime \prime}$. It is zero for $\mathrm{t}>\mathrm{t}^{\prime \prime}$. Here $\mathrm{e}_{\mathrm{o}}$ is termed the pulse height.

In a regulartriangular pulse $R^{\prime}=R^{\prime \prime}=R$. Such a pulse is shown in the center portion of the left half of Fig. 4. The excitation is

$$
\&(t)=R\left[t h(t)-2\left(t-t^{\prime}\right) h\left(t-t^{\prime}\right)+\left(t-2 t^{\prime}\right) h\left(t-2 t^{\prime}\right)\right]
$$

which transforms to

$$
\bar{\delta}(s)=\frac{R}{s^{2}}\left(1-2 \exp -t^{\prime} s+\exp -2 t^{\prime} s\right)=\frac{R}{s^{2}}\left(1-\exp -t^{\prime} s\right)^{2}
$$

Half of the second term in the center portion of Eq. (21) annihilates the effect of the first term. The third term counteracts the effect of the second half of the second term for $t>2 t^{\prime}$.

From Eq. (10) we obtain

$$
\bar{R}(s)=\frac{R \bar{m}(s)}{s}\left(1-2 \exp -t^{\prime} s+\exp -2 t^{\prime} s\right)
$$




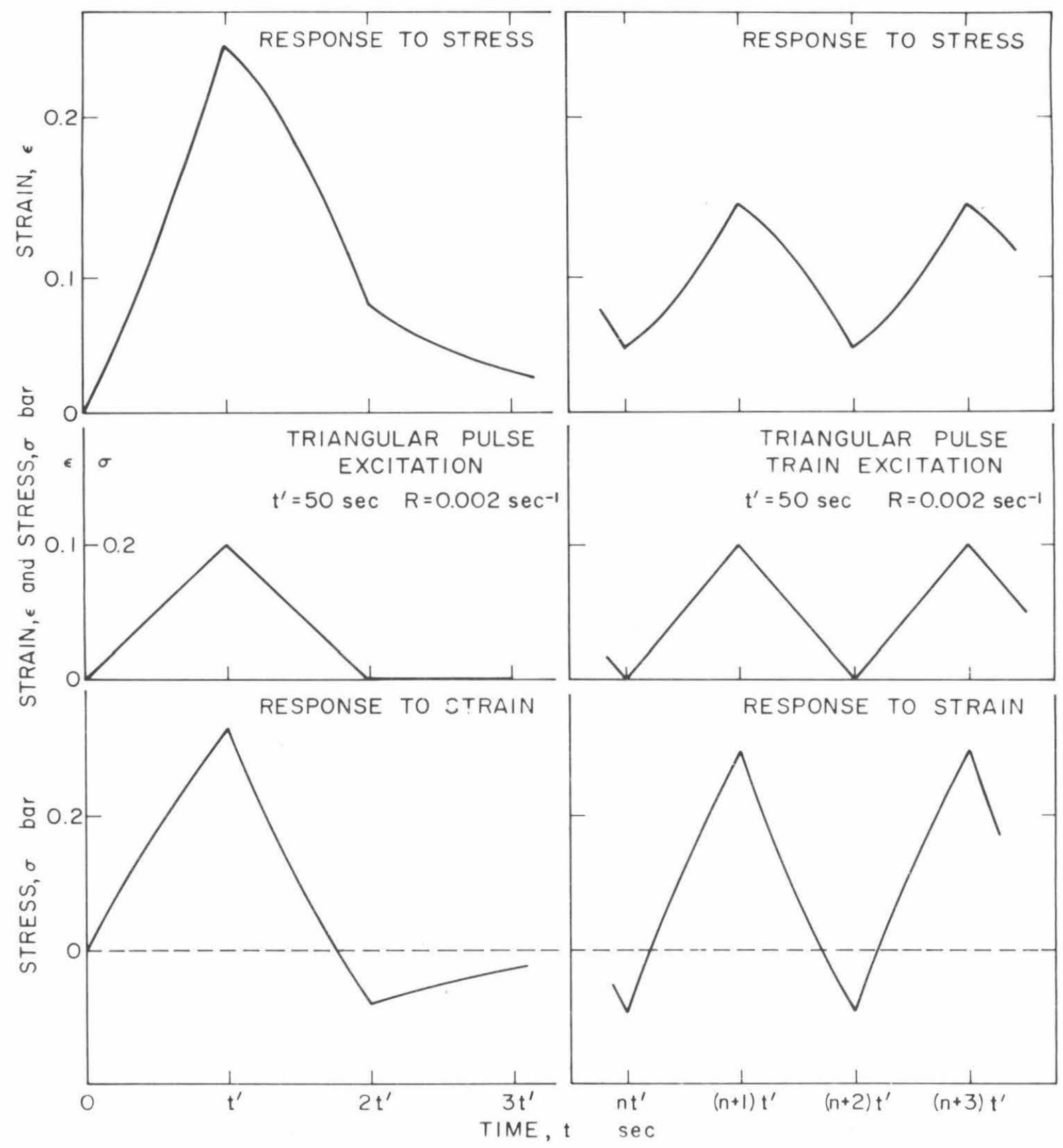

Fig. 4. Triangular Single Pulse and Pulse Train Excitation and Response. 
Specializing gives

$$
\begin{aligned}
\sigma(t)=R_{\varepsilon}\left\{\left[\int_{0}^{t} G(u) d u\right] h(t)\right. & -2\left[\int_{0}^{t-t^{\prime}} G(u) d u\right] h\left(t-t^{\prime}\right) \\
& \left.+\left[\int_{0}^{t-2 t^{\prime}} G(u) d u\right] h\left(t-2 t^{\prime}\right)\right\}
\end{aligned}
$$

and

$$
\begin{aligned}
\varepsilon(t)=R_{\sigma}\left\{\left[\int_{0}^{t} J(u) d u\right] h(t)\right. & -2\left[\int_{0}^{t-t^{\prime}} J(u) d u\right] h\left(t-t^{\prime}\right) \\
& \left.+\left[\int_{0}^{t-2 t^{\prime}} J(u) d u\right] h\left(t-2 t^{\prime}\right)\right\}
\end{aligned}
$$

where $R_{\varepsilon}$ and $R_{\sigma}$ are the rate of strain and rate of stress, respectively, and use has been made of Transform Pair (8) in the transform inversion. The responses to the three-parameter model are shown in the top and bottom part of the left side of Fig. 4. At $t=2 t^{\prime}$, when the stress is completely removed, the strain is still finite but gradually relaxes to zero (top part). When the excitation is a triangular strain pulse (bottom part), the material is actually in compression during the latter part of the second half of the interval of excitation for this particular choice of model. The recovery of the compression is retarded but eventually reaches zero.

\section{Triangular Pulse Train}

The triangular pulse train excitation is composed of a succession of triangular pulses as shown in the center part of the right side of Fig. 4. 
For a regular pulse the period is $2 t^{\prime}$. Applying the Periodicity Theorem, Eq. (3), to Eq. (22) gives

$$
\bar{\delta}(s)=\frac{R\left(1-\exp -t^{\prime} s\right)^{2}}{s^{2}\left(1-\exp -2 t^{\prime} s\right)}=\frac{R}{s^{2}}\left[1+2 \sum_{n=1}^{\infty}(-1)^{n} \exp -n t^{\prime} s\right]
$$

The right hand side of Eq. (26) is obtained from the center expression upon dividing and collecting terms.

The responses obtained by inserting Eq. (26) into Eq. (10) are

$\sigma(t)=R_{\varepsilon}\left\{\left[\int_{0}^{t} G(u) d u\right] h(t)+2 \sum_{n=1}^{\infty}(-1)^{n}\left[\int_{0}^{t-n t^{\prime}} G(u) d u\right] h\left(t-n t^{\prime}\right)\right\}$

for a strain excitation, and

$\varepsilon(t)=R_{\sigma}\left\{\left[\int_{0}^{t} J(u) d u\right] h(t)+2 \sum_{n=1}^{\infty}(-1)^{n}\left[\int_{0}^{t-n t^{\prime}} J(u) d u\right] h\left(t-n t^{\prime}\right)\right\}$

for a stress excitation.

Expressing $G(t)$ as a Prony series ${ }^{(5)}$ we have

$G(t)=G_{e}+\sum_{i=1}^{K} G_{i} \exp -t / \tau_{i}$

where $G_{e}$ is the equilibrium modulus. Substitution of Eq. (29) into Eq. (27) yields 


$$
\begin{gathered}
\sigma(t)=R_{\varepsilon}\left\{G_{e}\left[\operatorname{th}(t)+2 \sum_{n=1}^{\infty}(-1)^{n}\left(t-n t^{\prime}\right) h\left(t-n t^{\prime}\right)\right]+\sum_{i=1}^{K} G_{i} \tau_{i}\left(1-\exp -t / \tau_{i}\right) h(t)\right. \\
\left.+2 \sum_{n=1}^{\infty}(-1)^{n} \sum_{i=1}^{K} G_{i} \tau_{i}\left[1-\exp -\left(t-n t^{\prime}\right) / \tau_{i}\right] h\left(t-n t^{\prime}\right)\right\}
\end{gathered}
$$

The response to an arbitrary half-cycle, $\sigma_{N}(t)$, is obtained by summing $\mathrm{n}$ to $\mathrm{N}$ instead of $\infty$. Reversing the order of summation in the last term of Eq. (30), and summing on $\mathrm{n}$ yields the expressions

$$
\begin{aligned}
& 1+2 \sum_{n=1}^{N}(-1)^{n}=(-1)^{N} \\
& t+2 \sum_{n=1}^{N}(-1)^{n}\left(t-n t^{\prime}\right)=(-1)^{N} t+\frac{1-(2 N+1)(-1)^{N}}{2} t^{\prime}
\end{aligned}
$$

and

$$
2 \sum_{n=1}^{N}(-1)^{n} \exp n t^{\prime} / \tau_{i}=\frac{1-(-1)^{N} \operatorname{exp~Nt^{\prime }/\tau _{i}}}{1+\exp t^{\prime} / \tau_{i}} \exp t^{\prime} / \tau_{i}
$$

When $t$ is large, we obtain, using Eqs. (31), (32), and (33)

$$
\sigma_{N}(t)=\frac{G_{e} t^{\prime}}{2}-(-1)^{N}\left\{\frac{G_{e} t^{\prime}}{2}+G_{e} \theta+\sum_{i=1}^{K} G_{i} \tau_{i}\left[1-\frac{2 \exp -\theta / \tau_{i}}{1+\exp t^{\prime} / \tau_{i}}\right]\right\}
$$

where

$$
\theta=t-(N+1) t^{\prime}
$$


We can obtain the full steady-state response, $\sigma_{s s}(t)$, by setting

$$
\theta=\operatorname{th}(t)+2 \sum_{n=1}^{\infty}(-1)^{n}\left(t-n t^{\prime}\right) h\left(t-n t^{\prime}\right)
$$

where, of course, $t$ in Eqs. (35) and (36) are unrelated.

The steady-state responses of the three-parameter model are shown in Fig. 4. It is seen that for the particular choice of model, in the steady state the response to stress never reaches zero and the response to strain is a compressive stress during the latter part of the second halfcycle.

$$
\begin{aligned}
& \text { If } \\
& \delta(t)=\delta\left(t+2 n t^{\prime}\right)
\end{aligned}
$$

i. e. $\delta(t)$ is a periodic function of period $2 t^{\prime}$, and

$$
\bar{\delta}(s)=\bar{m}(s) / \bar{n}(s)
$$

i. e. the transform is the ratio of two polynomials, the numerator being of lower order than the denominator, then the steady-state response may be obtained from the Expansion Theorem ${ }^{(4)}$ as

$$
R_{s s}(t)=\sum_{k=1}^{N} \frac{\bar{S}\left(s_{k}\right) m\left(s_{k}\right)}{n\left(s_{k}\right)} \operatorname{exp~s_{k}t}
$$

where $\overline{\mathrm{S}}(\mathrm{s})$ is the system function, and the $\mathrm{s}_{\mathrm{k}}{ }^{\prime} \mathrm{s}$ are the zeros of $\overline{\mathrm{n}}(\mathrm{s})$. The double subscript on $R_{\mathrm{ss}}(t)$ indicates that the response obtained from Eq. is the steady state response. If the transients are to be included it is 
necessary to consider the poles of $\overline{\mathrm{S}}(\mathrm{s}) \bar{\xi}(\mathrm{s})$ rather than those of $\bar{\xi}(\mathrm{s})$ alone. *

The expression for the transform of the triangular pulse train excitation given by Eq. (26) may be rewritten

$$
\bar{\delta}(s)=\frac{R \sinh t^{\prime} s / 2}{s^{2} \cosh t^{\prime} s / 2}
$$

by an elementary transformation. Letting this be a strain excitation we obtain for the stress transform

$$
\bar{\sigma}(s)=\frac{R \bar{Q}(s) \sinh t^{\prime} s / 2}{s^{2} \cosh t^{\prime} s / 2}
$$

where $\bar{Q}(\mathrm{~s})$ is the operational relaxance ${ }^{(7)}$ defined by

$$
\bar{\sigma}(s)=\bar{Q}(s) \bar{\varepsilon}(\mathbf{s})
$$

The zeros of $\overline{\mathrm{n}}(\mathrm{s})$ are supplied by the solution of the equation

$$
\cosh \frac{t^{\prime} s_{k}}{2}=0
$$

This has only imaginary roots. Using the notation $s_{k}=j \omega_{k}$ we have

$$
\cosh \frac{j \omega_{k} t^{\prime}}{2}=\cos \frac{\omega_{k} t^{\prime}}{2}=0
$$

* Equation (39) must be modified for multiple zeros but this is not relevant here. Conditions other than the above mentioned ones are that the $\mathrm{s}_{\mathrm{k}}{ }^{\prime} \mathrm{s}$ must not be zeros of $\overline{\mathrm{n}}{ }^{\prime} \mathrm{s}$ and that $\bar{R}(\mathrm{~s})$ approach zero as $\mathrm{s}$ approaches infinity. Both conditions are fulfilled in the present context. 
from which

$$
\frac{\omega_{k} t^{\prime}}{2}=k \pi / 2, \quad k= \pm 1, \pm 3, \cdots
$$

since $\omega=\pi / t^{\prime}$. The required zeros become

$$
\mathrm{s}_{\mathrm{k}}=\mathrm{j} \omega_{\mathrm{k}}=\mathrm{jk \omega} \quad \mathrm{k}= \pm 1, \pm 3, \cdots
$$

Differentiation, making use of Eq. (43), yields

$$
\bar{n}^{\prime}(s)=\frac{t^{\prime} s^{2}}{2} \sinh t^{\prime} s / 2
$$

and

$$
\mathrm{n}^{\prime}\left(\mathrm{s}_{\mathrm{k}}\right)=\frac{\pi^{2} \mathrm{k}^{2}}{2 \mathrm{t}^{\prime}} \sinh \mathrm{j} \omega \mathrm{kt} \mathrm{t}^{\prime} / 2
$$

Applying Eq. (30), we find

$$
\sigma_{s s}(t)=-\frac{2 R t^{\prime}}{\pi^{2}} \sum_{k= \pm 2, \cdots}^{\infty} \frac{Q(j k \omega) \exp j \omega k t}{k^{2}}
$$

Rewriting Eq. (49) to indicate summation over positive values of $\mathrm{k}$ only, we obtain the stress in the steady state as

$$
\sigma_{s s}(t)=-\frac{2 \text { eo }}{\pi^{2}} \sum_{k=1,3 \ldots}^{\infty} \frac{1}{R^{2}}[Q(j k \omega) \exp j \omega k t+Q(-j k \omega) \exp -j \omega k t]
$$

where $e_{0}=R t^{\prime}$ is the pulse height. 
But $^{(7)}$

$$
Q(j k \omega)=G^{\prime}(k \omega)+j G^{\prime \prime}(k \omega)
$$

where $G^{\prime}(\omega)$ and $G^{\prime \prime}(\omega)$ are the storage and loss moduli. Remembering that $G^{\prime}(\omega)$ is an even, and $G^{\prime \prime}(\omega)$ an odd function of $\omega$, we finally have

$$
\left.\sigma_{\mathrm{ss}}(\mathrm{t})=\frac{4 \mathrm{e}_{0}}{\pi^{2}} \sum_{\mathrm{k}=1,3 \ldots \mathrm{k}^{2}}^{\infty} \frac{1}{\mathrm{k}^{\prime \prime}}\left(\frac{\mathrm{k} \pi}{\mathrm{t}}\right) \sin \frac{\mathrm{k} \pi \mathrm{t}}{\mathrm{t}^{\prime}}-\mathrm{G}^{\prime}\left(\frac{\mathrm{k} \pi}{\mathrm{t}^{\prime}}\right) \cos \frac{\mathrm{k \pi t}}{\mathrm{t}^{\prime}}\right]
$$

The steady state response to a triangular stress excitation is obtained in an analogous way as

$$
\epsilon_{s s}(t)=-\frac{4 e_{o} t^{\prime}}{\pi^{2}} \sum_{k=1,3 \ldots}^{\infty}\left[J^{\prime}\left(\frac{k \pi}{t}\right) \cos \frac{k \pi t}{t^{\prime}}+J^{\prime \prime}\left(\frac{k \pi}{t}\right) \sin \frac{k \pi t}{t^{\prime}}\right]
$$

in terms of the storage and loss compliances.

\section{Other Piecewise Continuous Excitations}

The preceding sections have outlined a general approach for the mathematical treatment of some of the simpler piecewise continuous excitations. More complicated excitations can be treated in a similar way. A great many "repeat" functions, $f_{t}(t)$, have been tabulated. They are listed pictorially with the corresponding Laplace transforms in such books as that by Fodor ${ }^{(4)}$ and by Doetsch ${ }^{(8)}$. The periodic functions can be found from them by applying the Periodicity Theorem as demonstrated in this report. The task of describing the behavior of linearly viscoelastic materials in terms of standard excitations is thus greatly facilitated. 


\section{REFERENCES}

1. J. C. Halpin, J. Appl. Phys. $\underline{36}: 2675$ (1965).

2. T. Tanaka and T. L. Smith, unpublished results.

3. R. V. Churchill, Modern Operational Mathematics in Engineering, McGraw-Hill, New York, 1944, p. 4.

4. G. Fodor, Laplace Transforms in Engineering, English Translation, Hungarian Academy of Sciences, Budapest, 1965.

5. R. A. Schapery, Proc. Fourth U.S. Nat. Congress Appl. Mech., Vol. 2, p. 1075, ASME, New York, 1962.

6. H. Leaderman, Trans. Soc. Rheol. 6:361 (1962).

7. N. W. Tschoeg1, Kolloid-Zeitschrift, 174, 113 (1961).

8. G. Doetsch, Anleitung zum praktischen Gebrauch der Laplace Transformation, Oldenburg, Munich, 1961. 


\section{CPIA DISTRIBUTION}

Bureau of Mines

Attn: ERC Library

4800 Forbes Avenue

Pittsburgh, Pennsylvania 15213

Central Intelligence Agency

Attn: CRS/ADD - Standard Dist.

Washington, DC 20505

NASA

Lewis Research Center

Attention: Librarian

21000 Brookpark Road

Cleveland, Ohio 44135

NASA

Kennedy Space Center

Attn: Library-ATS-132C

Kennedy Space Center

Florida 32899

NASA

Manned Spacecraft Center

Attn: Library/Code BM6

Houston, Texas 77058

NASA

Langley Research Center

Attention: Librarian

Langley Station

Hampton, Virginia 23565

NASA

Scientific and Technical

Information Facility

Attn: SAF/DL, ACQ Division

P. O. Box 33

College Park, Maryland 20740

NASA

Attn: Code RPS, R. W. Ziem Washington, DC 20546
$1 \quad$ NASA

Goddard Space Flight Center

Attention: Library, Code 252

Greenbelt, Maryland 20771

1 Defense Documentation Center 20 Attention: TSR

Cameron Station, Building 5

Alexandria, Virginia 22314

Air Force Systems Command 1 Attention: SCTSP

Andrews Air Force Base

Washington, DC 20331

$1 \quad$ Arnold Engineering

Development Center

Attention: AEOIM

Arnold Air Force Station

Tennessee 37389

Air Force Rocket Propulsion Lab

Attention: RPC

Edwards, California 93523

3 Air Force Rocket Propulsion 1 Lab

Attention: RPM

Edwards, California 93523

Air Force Flight Test Center 1

2 Attn: FTBPP-2, Tech Library

Edwards Air Force Base

California 93523

Air Force Office

of Scientific Research

Attention: SREP

1400 Wilson Boulevard

Arlington, Virginia 22209

AFRPL

Attention: Technical Library

Edwards, California 93523 
Foreign Technology Division

Attention: TDBTL

Wright-Patterson Air Force Base Ohio 45433

Army Ballistic Research Labs 1 Attention: AMXBR-1

Aberdeen Proving Ground

Maryland 21005

Army Research Office

Attention: CRD-AA-IP

Box CM, Duke Station

Durham, North Carolina 27706

Frankford Arsenal

Attn: C2500-Library-B 51-2

(for Propellant and Expl. Section)

Philadelphia, Pennsylvania 19137

Picatinny Arsenal

Scientific and Technical

Information Branch

Attn: SMUPA-VA6, Librarian

Dover, New Jersey 07801

Army Missile Command

Redstone Scientific Info Center

Attn: Chief, Document Section

Redstone Arsenal, Alabama 35809

White Sands Missile Range

Attention: Technical Library

1

White Sands MR, New Mexico 88002

Naval Air Systems Cmd.

Attention: AIR-604

Washington, DC 20360

Naval Air Systems Cmd.

AIR - 5367

Washington, DC 20360

Naval Air Systems Cmd.

Attention: AIR-5366

Washington, DC 20360

Naval Ordnance Systems Cmd. 2 Attention: ORD-0331

Washington, DC 20360
CPIA Distribution Page 2

1 Naval Missile Center

Attn: Code 5632. 2

Technical Library

Point Mugu, California 93041

Naval Ordnance Laboratory

Attention: Library

White Oak

Silver Spring, Maryland 20910

1 Naval Air Systems Cmd. . 2

Attention: AIR - 330

Washington, DC 20360

Naval Weapons Center

Attn: Code 753-Tech Library

China Lake, California 93555

Naval Postgraduate School 1

Attention: Library Tech Reports Section-2124

Monterey, California 93940

Naval Ordnance Station

Attention: Technical Library

Indian Head, Maryland 20640

Naval Research Branch Office I

Attention: Librarian

1030 East Green Street

Pasadena, California 91101

Naval Research

1

Power Branch

2 Washington, DC 20360

Naval Ordnance Systems Cmd. 2 Attention: ORD-9132

2 Technical Library

Washington, DC 20360

Naval Special Projects

1 Attention: Technical Library

Washington, DC 20360

Naval Underwater Weapons

Research Engineering Station Attn: Technical Library (CS12)

Newport, Rhode Island 02840 
Aerojet-General Corporation Attention: Technical Library P. O. Box 296

Azusa, California 91702

Aerojet-General Corporation Attention: Library 11711 South Woodruff Avenue Downey, California 90241

Aerojet-General Corporation Attention: Technical Library 2432-2015A

P. O. Box 15847

Sacramento, California 95813

Philco-Ford Corporation

Aeronutronic Division

Attention: Technical Information

Svcs. Acquisitions

Ford Road

Newport Beach, California 92663

Garrett Corporation

Airesearch Mfg. Division

Attention: Technical Library

9851 Sepulveda Boulevard

Los Angeles, California 90009

Aerospace Corporation

Attention: Technical Information

Center Document Group

P. O. Box 95085

Los Angeles, California 90045

Allied Chemical Corporation

Attention: Security Officer

P. O. Box 70

Morristown, New Jersey 07960

IIT Research Institute

Attention: Document Library

10 West 35 th Street

Chicago, Illinois 60616

ARO, Incorporation

Attn: Technical Documents Library Arnold Air Force Station

Tennessee 37389

1

1

3

CPLA Distribution Page 3

Atlantic Research Corporation 2

Attention: Library

Shirley Highway at Edsall Road

Alexandria, Virginia 22314

University of Denver

Denver Research Institute

Attention: Security Officer

P. O. Box 10127

Denver, Colorado 80210

Bell Aerosystems Company

Attention: Technical Library

P. O. Box 1

Buffalo, New York 14240

1 Boeing Company

Attention: Aerospace Library $8 \mathrm{~K}-38$

P. O. Box 3999

Seattle, Washington 98124

Chemical Propulsion Information Agency

Applied Physics Lab-JHU

8621 Georgia Avenue

Silver Spring, Maryland 20910

Lockheed Missiles and Space Company

Attn: Tech Info Ctr. 50-14

3251 Hanover Street

Palo Alto, California 94304

McDonnell Douglas Corporation 1

1 Douglas Missile and Space Systems Division

Attention: Library A2-260 3000 Ocean Park Boulevard

Santa Monica, California 90406

Dow Chemical Company

Scientific Projects Laboratory

Attn: R. S. Karpiuk, Bldg 1710

Midland, Michigan 48641

Esso Research and Engineering 1 Company

Process Research Division

Attention: Dr. J. R. Lovett

Linden, New Jersey 07036 
General Dynamics Corporation 1 Attention: Library

P. O. Box 2507

Pomona, California 91766

General Dynamics/Convair Library and Information Services P. O. Box 12009

San Diego, California 92112

Hercules, Incorporation

Allegany Ballistics Lab

Attention: Technical Library

P. O. Box 210

Cumberland, Maryland 21502

Hercules, Incorporation

\section{1}

Research Center

Attention: Technical Information Center

Wilmington, Delaware 19899

Institute for Defense Analyses Attention: Classified Library 400 Army-Navy Drive

Arlington, Virginia 22202

California Institute of Technology

Jet Propulsion Laboratory

Attention: Library, TDS

4800 Oak Grove Drive

Pasadena, California 91103

Lockheed Propulsion Company

Attention: Library

P. O. Box 111

Redlands, California 92373

Marquardt Corporation

Attention: Library

P. O. Box 2013, South Annex

Van Nuys, California 91409
CPIA Distribution Page 4

New York University

School of Engineering and

Science

Attention: Research Division

P. W. Winternitz

1 West 181 Street and University Ave.

Bronx, New York 10460

North American Rockwell

Space and Information

Systems Division

Attention: Tech Info Ctr-096-722

12214 Lakewood Boulevard

Downey, California 90241

Rocket Research Corporation l

Attention: Technical Library

520 South Portland Street

Seattle, Washington 98198

North American Rockwell

Rocketdyne Division

1 Attn: Library Department 086-306

6633 Canoga Avenue

Canoga Park, California 91304

Rohm and Haas Company

1 Redstone Research Labs

Attention: Technical Library

Huntsville, Alabama 35807

TRW Systems, Incorporation 1

Attention: Technical Information C enter

2 One Space Park

Redondo Beach, California 90278

Texaco Experiment, Inc.

Attention: Librarian

$1 \quad$ P. O. Box 3407

Richmond, Virginia 23202

Thiokol Chemical Corporation 1

Huntsville Division

Attention: Technical Library

Huntsville, Alabama 35807 
Thiokol Chemical Corporation 1 Elkton Division

Attention: Tech Info Center

Elkton, Maryland 21921

Thiokol Chemical Corporation Wasatch Division

Attention: Technical Library

Brigham City, Utah 84302

Stanford Research Institute

Document Center

For Propulsion Sciences

333 Ravenswood Avenue

Menlo Park, California 94025

United Aircraft Corporation

Attn: Acquisitions Librarian 400 Main Street

East Hartford, Connecticut 06108

United Aircraft Corporation

Pratt and Whitney Aircraft Div.

Attention: Head Librarian

P. O. Box 2691

West Palm Beach, Florida 33402

United Technology Center

Attention: Technical Library

P. O. Box 358

Sunnyvale, California 94988

General Electric Company

Attention: Acquisitions

Building 4, Room 109

Daytona Beach, Florida 32015

Dupont Company

Eastern Division

Attention: Report Clerk

A. R. Steward

Gibbstown, New Jersey 08027

Thiokol Chemical Corporation

Reaction Motors Division

Attention: Library

Denville, New Jersey 07834

2

1
CPIA Distribution Page 5

Princeton Univer sity 1

Forrestal Campus Library

Attention: Librarian

P. O. Box 710

Princeton, New Jersey 08540

Defense Rsch, and Engrg.

Pentagon, 3D1065

Washington, DC 20301

Attention: Propulsion Technology

Army Ammo. Procurement 1 and Supply Agency

Attention: RDL, Quality Assurance Technical Library

Joliet, Illinois 60431

Air Force Rocket

Propulsion Lab

Attention: RPCE

Edwards, California 93523

Callery Chemical Company

Research and Development Div.

Attention: Document Control

Callery, Pennsylvania 16024

1 McDonnell Douglas Corporation 1

Attention: Library

P. O. Box 516

St. Louis, Missouri 63166

1 Ethyl Corporation

1

P. O. Box 53091

Baton Rouge, Louisiana 70805

Attention: Program Manager

1 Hercules, Inc.

1

Bacchus Works

Attention: Library $100-\mathrm{H}-2$

P. O. Box 98

Magna, Utah 84044

1 Olin Mathieson Chemical

Corporation

Attention: Research Library

P. O. Drawer G

Marion, Illinois 62959 
Minnesota Mining and

Manufacturing Company

2501 Hudson Road

St. Paul, Minnesota 55119

Attention: Security Officer

H. C. Zeman

Olin Mathieson Chemical

Corporation

Mail Control Room

Attention: Asst. Librarian

275 Winchester Avenue

New Haven, Connecticut 06504

Pennsalt Chemicals Corporation 1 Technological Center

Attention: Security Officer

900 First Avenue

King of Prussia, Pennsylvania

19406

Purdue University

School of Mechanical Engineering

Attention: B. A. Reese

Lafayette, Indiana 47907

North American Rockwell

Rocketdyne Division

Attention: Library

McGregor, Texas 76657

Shell Oil Company

Shell Development Company

Division

Attention: Technical Files

1400 53rd Street

Emeryville, California 94608

Aerospace Corporation

Attention: Library

P. O. Box 1308

San Bernardino, California 92402

University of Utah

Attention: Security Officer

(for M. L. Williams)

Salt Lake City, Utah 84112
CPIA Distribution Page 6

1 Los Alamos Scientific Lab

Attention: Report Library

P. O. Box 1663

Los Alamos, New Mexico 87544

Foreign Technology Division 1

Attention: TDPMP

1 Wright-Patter son AFB

Ohio 45433

AFRPL (RPMC)

Attention: Dr. F. N. Kelley

Edwards Air Force Base

California 93523

AFRPL (RPMCB)

Attention: Lt. S. W. Beckwith

Edwards Air Force Base

California 93523

AFRPL (RPX)

Attention: Dr. L. G. Edwards Edwards Air Force Base

California 93523

AFRPL (RPCCR)

1 Attention: Dr. C. Merrill

Edwards Air Force Base

California 93523

Plastech Corporation

1 Attention: Mr. Fred Singdale

Natick, Massachusetts 01760

Texas $A$ and $M$ University

Attention: D. Webb

College Station, Texas 77843

1 CETEC

188 Whisman Road

Attention: R. Beyer

Mountain View, California 94040 


\section{DOCUMENT CONTROL DATA - R \& D}

(Security classification of title, body of abstract and indexing annotation must be entered when the overall report is classified) 1. ORIGINATING ACTIVITY (Corporate author)

Air Force Rocket Propulsion Laboratory

Research and Technology Division

Edwards Air Force Base, California 93523

2a. REPORT SECURITY CLASSIFICATION

3. REPORT TITLE

A RESEARCH PROGRAM ON SOLID PROPELLANT PHYSICAL BEHAVIOR

4. DESCRIPTIVE NOTES (TYPe of report and inclusive dates)

First Annual Report, 1 February 1967 - 30 April 1968

5. AUTHOR(S) (First name, middle initial, last name)

Nicholas W. Tschoegl, Robert F. Landel, Henning Kausch, Chun K. Lim, Ralph G. Mancke, Donald G. Fesko, Keikichi Yagii

\begin{tabular}{|c|c|c|}
\hline $\begin{array}{l}\text { 6. REPORT DATE } \\
\text { June } 1968\end{array}$ & $\begin{array}{l}\text { 7a. TOTAL NO. OF PAGES } \\
229\end{array}$ & $\begin{array}{c}\text { 7b. NO. OF REFS } \\
137\end{array}$ \\
\hline Ba. CONTRACT OR GRANT NO. & \multirow{2}{*}{\multicolumn{2}{|c|}{ 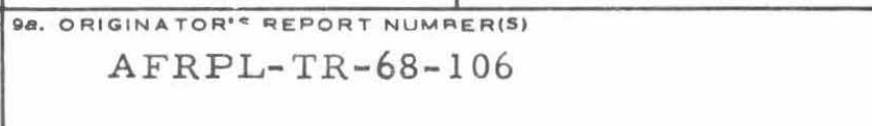 }} \\
\hline $\begin{array}{l}\text { F04611-67-C-0057 } \\
\text { b. PROJECTNO. }\end{array}$ & & \\
\hline 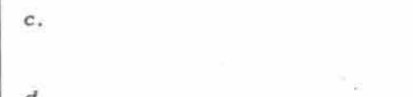 & $\begin{array}{l}\text { 9b. OTHER REPORT NO(S) } \\
\text { this roport) } \\
\text { CHECIT PL }\end{array}$ & her numbers that may be assign \\
\hline
\end{tabular}

10. DISTRIBUTION STATEMENT

This document is subject to special export controls and each transmittal to foreign governments or foreign nationals may be made only with prior approval of

AFRPL(RPPR-STINFO), Edwards, California 93523

11. SUPPLEMENTARY NOTES

12. SPONSORING MILITARYACTIVITY
AFFTC, Directorate of Procurement
(FTMKR-3)
Edwards Air Force Base, California 93523

13. ABSTRACT

Part I reports on further progress in a program to generate failure data on filled and unfilled elastomers in the compressive octants of principal stress space. Rubber rings swollen in hydrocarbon solvents were stretched in uniaxial tension at a crosshead speed of 1.45 inches per minute under superposed hydrostatic pressures up to 2000 psig. Two elastomers were examined: a moderately cross-linked compression molded styrene-butadiene rubber, and the interim standard rubber, GA LCIT I, a more tightly cross-linked cast polyurethane rubber. Both materials obeyed the stress strain relation predicted by the kinetic theory of rubber elasticity under all pressures. The tests were carried out at room temperature and were corrected to $25^{\circ} \mathrm{C}$. The superposed hydrostatic pressure had no effect on the tensile strength of the two rubbers. Within the experimental scatter the stress-at-break appeared to depend on the octahedral shear stress alone and thus followed the Huber-Hencky-von Mises failure criterion. The failure surface of the swollen rubbers in principal stress space could therefore be described by a cylinder coaxial with the hydrostatic axis.

Part II reports on an attempt to derive the optimal distribution of sizes of randomly aggregated spheres as a first step in the development of a theory of optimum packing. It is shown that an investigation of the interstices in a lattice of regularly packed spheres does not yield an unambiguous distribution over a wide range of sphere sizes. The corresponding problem in the plane, however, could be studied unambiguously. The results obtained in such a study are useful because of the equivalence of volume, area, and line fractions of random assemblies. An optimal distribution of circle sizes, $\mathrm{n}(\mathrm{r})$, for the regular packing of circles in a plane was derived. The value of $\mathrm{n}(\mathrm{r})$ in random packing of circles is discussed and a method 


\section{UNCLASSIFIED}

\section{DD Formi 1473 - Abstract cont'd.}

is developed to transform $\mathrm{n}(\mathrm{r})$ into the corresponding distribution $\mathrm{N}(\mathrm{R})$ of sphere sizes. $N(R)$ is obtained in form of a histogram. It still remains to be shown experimentally whether sphere distributions of the calculated type do indeed result in maximum packing fractions and optimize mechanical properties of filled elastomers.

A research program to investigate the blending of two homopolymers with the aid of diblock copolymers is outlined in Part III. The thermodynamics of the separation of polymers in blends, and the synthesis of block copolymers by means of anionic polymerization are discussed. The program is to include microscopic examination of a sequence of ternary mixtures of two homopolymers with their diblock copolymer to determine the variation in domain size of the separated components. The blends will then be characterized mechanically by measuring the dynamic shear modulus. These studies are expected to contribute to our knowledge of binderfiller interaction, and may lead to novel ways of binder formulation.

Part IV deals with characterization studies in the area of the standardized rubber program. The measurements of the physical properties of the Solithane 113 elastomers, including the interim standard rubber, GA LCIT I, were continued. Sol-gel and compressive stress-strain measurements in the swollen state showed that there was little sol at any composition from 50 to 80 volume percent of prepolymer and that the effective chain concentration was a constant over this composition range. A further analysis of the ultimate properties measured in uniaxial extension, in conjunction with these results, showed that the increase in prepolymer content lowers the number of equivalent random links per chain from 12 to 4.2. The polymersolvent interaction parameter $\chi$, was evaluated as a function of the degree of swelling in five solvents. The solubility parameter was found to be $11.6\left(\mathrm{cal} / \mathrm{cm}^{3}\right)^{\frac{1}{2}}$. The Mooney-Rivlin $\mathrm{C}_{z}$ parameter is essentially zero at room temperature. An independent measurement of Poisson's ratio as determined in the poker chip test at 5\% compressive strain gave a value of 0.4996 , which compares reasonably well with a previous value of 0.4998 .

Part V reports on finite deformation studies. Large-strain properties of natural rubber cross-linked with $1 \%$ sulfur were studied in an attempt to further our understanding of the strain-energy function of that material. The Becker-Landel torsion-tension tester was used to measure the torsional and axial forces required to support combined twist and extension, or compression, of more than 50 solid cylindrical rubber specimens of three different radii. In the cases of combined extension and twist the forces at five minutes after application of the deformation were found to follow equations derived from the Signorini form of the strain-energy function, with appropriate values of the three material constants. Further work showed. however, that the values of the twist variable were in error to some extent, and further discussion will be deferred until correction of these errors.

Part VI describes a continuation of earlier investigations of approximations to the spectral distribution functions of linear viscoelastic theory. It is shown that if the order of the highest derivative is $k$, then there exist $k$ approximations from the step and the storage functions, and $k+1$ approximations from the loss functions. Thus a number of new, hitherto unknown, approximations are found, which include approximations of even cont'd 


\section{UNCLASSIFIED}

DD Form 1473 - Abstract cont'd.

order from the storage functions, and approximations of odd order from the loss functions. Of particular practical interest are the two approximations of first order from the loss functions. The inter-relation between the $k$ approximations of a given order, and the choice of the most suitable approximation is discussed. The relation of the more general method to the transform inversion method is examined, and it is shown that certain approximations in the current literature are in error because of lack of normalization of the associated intensity function, and, where applicable, because of failure to take into account that the time scale of the spectrum must be shifted by the appropriate amount if the maximum of the intensity function does not lie at the origin. Finally, the derivation of approximations from creep recovery, stress relaxation after cessation of steady flow, and deformation at constant rates of strain is discussed.

In Part VII the $\mathrm{z}$-transform method for the numerical inversion of the Laplace transform is examined for possible application to viscoelastic problems. The method is found to be unsuited for viscoelastic functions which are given as logarithmic functions of time or frequency, because the logarithmic spacing of the variables leads to unstable solutions.

Part VIII predicts a general method for expressing the response of viscoelastic materials to the piecewise continuous excitation functions to which they are subjected in cumulative damage testing, as functions of the standard response functions such as relaxation modulus and creep compliance, or, in case of periodic excitations, the dynamic moduli and compliances. It is expected that departures from the predicted behavior can be ascribed to cumulative damage. This could lead to the development of a non-destructive fatigue tests. 